\title{
Preoperative optimization of physical functioning in patients with colorectal cancer
}

Citation for published version (APA):

Heldens, A. F. J. M. (2019). Preoperative optimization of physical functioning in patients with colorectal cancer. [Doctoral Thesis, Maastricht University]. ProefschriftMaken.

https://doi.org/10.26481/dis.20191108ah

Document status and date:

Published: 01/01/2019

DOI:

10.26481/dis.20191108ah

Document Version:

Publisher's PDF, also known as Version of record

\section{Please check the document version of this publication:}

- A submitted manuscript is the version of the article upon submission and before peer-review. There can be important differences between the submitted version and the official published version of record.

People interested in the research are advised to contact the author for the final version of the publication, or visit the DOI to the publisher's website.

- The final author version and the galley proof are versions of the publication after peer review.

- The final published version features the final layout of the paper including the volume, issue and page numbers.

Link to publication

\footnotetext{
General rights rights.

- You may freely distribute the URL identifying the publication in the public portal. please follow below link for the End User Agreement:

www.umlib.nl/taverne-license

Take down policy

If you believe that this document breaches copyright please contact us at:

repository@maastrichtuniversity.nl

providing details and we will investigate your claim.
}

Copyright and moral rights for the publications made accessible in the public portal are retained by the authors and/or other copyright owners and it is a condition of accessing publications that users recognise and abide by the legal requirements associated with these

- Users may download and print one copy of any publication from the public portal for the purpose of private study or research.

- You may not further distribute the material or use it for any profit-making activity or commercial gain

If the publication is distributed under the terms of Article $25 \mathrm{fa}$ of the Dutch Copyright Act, indicated by the "Taverne" license above, 

PREOPERATIVE OPTIMIZATION OF PHYSICAL FUNCTIONING IN PATIENTS WITH COLORECTAL CANCER 
Layout and printing ProefschriftMaken | www.proefschriftmaken.nl

Cover design Ontwerpen met Kris | info@ontwerpenmetkris.nl

ISBN 978-94-6380-469-1

(C) Aniek Heldens, Maastricht

De kleuren op de cover zijn gekozen om de volgende betekenis:

Blauw Communicatie | Betrouwbaar | Stabiliteit

Geel Vernieuwend | Mentaal sterk | Bewegelijk

Groen Balans | Groei | Gezondheid

All rights reserved. No part of this publication may be reproduced or used in any manner whatsoever without prior written permission from the author. 


\title{
PREOPERATIVE OPTIMIZATION OF PHYSICAL FUNCTIONING IN PATIENTS WITH COLORECTAL CANCER
}

\author{
PROEFSCHRIFT \\ ter verkrijging van de graad van doctor aan de Universiteit Maastricht, \\ op gezag van de Rector Magnificus, Prof. dr. Rianne M. Letschert, \\ volgens het besluit van het College van Decanen, \\ in het openbaar te verdedigen op \\ vrijdag 8 november 2019 om 12.00 uur \\ door \\ Aniek Francisca Jacoba Maria Heldens
}




\section{Promotores}

Prof. dr. N.L.U. van Meeteren

Prof. dr. W.F.F.A. Buhre

\section{Copromotor}

Dr. B.C. Bongers

\section{Beoordelingscommissie}

Prof. dr. M.P. Weijenberg (voorzitter)

Prof. dr. N.D. Bouvy

Prof. dr. C.J. Kalkman (Universitair Medisch Centrum Utrecht)

Prof. dr. J.M. Klaase (Universitair Medisch Centrum Groningen)

Prof. dr. B. Kremer

The printing of this thesis was financially supported by Maastricht University and the Department of Physical Therapy of the Maastricht UMC+. 



\section{Paranimfen}

Wai Yan Liu

Christel van Beijsterveld 


\section{CONTENTS}

\section{Chapter 1}

General introduction

Chapter 2

Variation in preoperative and postoperative physical therapist management for patients opting for elective abdominal surgery

\section{Chapter 3}

The association between performance parameters of physical fitness and postoperative outcomes in patients undergoing colorectal surgery:

an evaluation of care data

\section{Chapter 4}

Clinical course of physical fitness and skeletal muscle mass during neoadjuvant chemoradiotherapy in patients with locally advanced rectal cancer:

an observational longitudinal study of 25 single subjects

\section{Chapter 5}

Feasibility and preliminary effectiveness of a physical exercise

training program during neoadjuvant chemoradiotherapy in individual patients with rectal cancer prior to major elective surgery

\section{Chapter 5 supplement}

Reply to: Exercising patient-centeredness in prehabilitation programs

\section{Chapter 6}

A one-group pre-test post-test pilot study to evaluate a supervised home-based prehabilitation program for high-risk patients undergoing major elective abdominal surgery: a study protocol

\section{Chapter 7}

General discussion

Valorization

Summary

Samenvatting

Dankwoord 

Chapter 1

General introduction 



\section{GENERAL BACKGROUND}

Advances in public health and medicine have led to improvements in overall life expectancy. Worldwide, people are getting older and the number of people above the age of 65 is still increasing. According to the World Health Organization, $6.9 \%$ of the world population was 65 or older in the year 2000 with an estimated increase to $10.4 \%$ in 2025 and a further foreseen rise up to $16.4 \%$ in 2050 [1]. With the increase of the aging population, the incidence of all cancer types, including colorectal cancer, will also rise. The worldwide incidence of colorectal cancer was 1.4 million in 2012 and is expected to be 2.4 million in 2035 [2]. Additionally, for all types of cancer more treatment modalities are available nowadays, which leads to improvements in survival. Formerly being lethal, cancer will become a chronic disease in the future, with the focus on living with the chronic disease.

\section{International background}

For patients with colorectal cancer, surgical interventions with or without (neo)adjuvant treatment - the prevailing cure strategy here - are more frequently performed; however, these interventions are associated with potential complications, resulting in an increasing demand on the health care system. In parallel, developments in medical health care are ongoing, ensuring continuous improvements in the perioperative period. Many innovations have already been made, and will continuously make surgery safer, more effective, and more applicable to a larger and more diverse population. The introduction of a national screening program will result in the increase of patients suitable for adequate therapy [3], followed by improvements in surgical care like minimally invasive surgery. Fast track programs, which contain various medical interventions that can promote recovery after surgery, as enhanced recovery after surgery (ERAS) are accepted evidence-based multimodal treatment concepts. These concepts in perioperative health care have a beneficial impact on recovery after (colorectal) surgery [4,5]. A meta-analysis incorporating twenty-five trials on ERAS programs for colorectal surgery showed a mean reduction in length of hospital stay of 2.6 days (95\% confidence interval, -3.2 to -2.0 days) in the ERAS group, compared to traditional care [6]. In addition, significant reductions in morbidity ( $15.1 \%$ with ERAS, $24.6 \%$ with standard treatment) and nonsurgical complications (3.0\% with ERAS, $7.5 \%$ with standard treatment) were found [7]. Despite these advances, the incidence of postoperative complications in colorectal surgery is still significant (17.0 and $24.0 \%$ ) [8]. 


\section{Chapter 1}

\section{Regional background}

The origin of the set up and execution of this pragmatic research project, as part of a nationwide program on perioperative care, is the combination of daily clinical physical therapy practice and applied science. To achieve this goal, physical therapists work both, in- and outside the hospital in an interdisciplinary and - later on - transmural team. In parallel, they act as embedded scientists to step-by-step change daily practice according to state-of-the-art knowledge and evidence [9]. Additionally, these scientists continuously gather new insights and knowledge by collecting, analyzing and interpreting routine data from patients during daily routines, and implement these insights in clinical care pathways [9]. By doing so, the connections between the patient journey, the developing health care system, and research and innovations are made with the overall goal to improve patient outcomes by optimizing the perioperative care pathway in joint coalition with the patients with colorectal cancer and their social support system.

\section{COLORECTAL CANCER}

Colorectal cancer, consisting of tumors in the tissues of the colon and the rectum, is the third most common diagnosis of cancer in the Netherlands [10], with an incidence of 14,090 in 2018. For most patients with localized colorectal cancer (rectal cancer; $99.0 \%$, colon cancer; $87.0 \%)$, the primary treatment consists of elective colorectal resection, with or without (neo)adjuvant treatment [8]. In the Netherlands, around $60.0 \%$ of the patients with rectal cancer is eligible for neoadjuvant therapy (radiotherapy and/or chemotherapy) prior to elective surgery [8]. Adjuvant chemotherapy is prescribed in $3.6 \%$ of the patients with rectal cancer and in $26.0 \%$ of the patients with colon cancer [8]. Figure 1 shows the clinical pathway and the people's journey for colorectal cancer. 


\begin{tabular}{|c|c|}
\hline \multirow[t]{3}{*}{$\begin{array}{l}\text { Clinical } \\
\text { pathway }\end{array}$} & People's journey \\
\hline & $\begin{array}{l}\text { Performing activities of daily } \\
\text { living, participating in society }\end{array}$ \\
\hline & $\begin{array}{l}\text { Receiving an invitation for the } \\
\text { national screening program }\end{array}$ \\
\hline General & $\begin{array}{l}\text { Possible problems with performing } \\
\text { activities of daily living }\end{array}$ \\
\hline Practitioner & \\
\hline \multirow{2}{*}{$\begin{array}{l}\text { Examination } \\
\text { In the hospital }\end{array}$} & \\
\hline & Performing activities of daily \\
\hline $\begin{array}{l}\text { Diagnosis } \\
\text { Colon cancer } \\
\text { Rectal cancer }\end{array}$ & $\begin{array}{l}\text { living, participating in society } \\
\text { Consulting (medical) specialists, } \\
\text { considering treatment options }\end{array}$ \\
\hline Treatment & Neoadjuvant treatment. \\
\hline $\begin{array}{l}\text { Neoadjuvant } \\
\text { treatment }\end{array}$ & $\begin{array}{l}\text { Experiencing possible side } \\
\text { effects of the treatment }\end{array}$ \\
\hline Surgery & Postoperative recovery of \\
\hline Clinical recovery & $\begin{array}{c}\text { physical functioning, engaging } \\
\text { in physical therapy treatment }\end{array}$ \\
\hline \multirow[t]{2}{*}{ Discharge } & \\
\hline & $\begin{array}{l}\text { Recovery, preparıng for adjuvant } \\
\text { treatment. Performing activities, } \\
\text { (partly) participating in society }\end{array}$ \\
\hline \multirow{3}{*}{$\begin{array}{l}\text { Adjuvant } \\
\text { treatment }\end{array}$} & Recovery \\
\hline & $\begin{array}{l}\text { Considering participation in a } \\
\text { rehabilitation program. Possible } \\
\text { problems in activities of daily living }\end{array}$ \\
\hline & $\begin{array}{c}\text { Experiencing possible disease- and } \\
\text { treatment related long-term side } \\
\text { effects }\end{array}$ \\
\hline
\end{tabular}

Figure 1. Clinical pathway and people's journey for colorectal cancer. 


\section{Chemotherapy and/or radiotherapy}

The medical treatment of chemotherapy and radiotherapy in addition to abdominal resection aims to control local tissue derangement and to improve resectability by downsizing the tumor and increasing negative resection margins [11,12]. However, neoadjuvant therapy is associated with negative side effects, such as diarrhea, hand-foot syndrome, and cardiotoxicity [13]. Additionally, chemoradiotherapy has side effects, of which fatigue [14] and a decrease in aerobic capacity $[15,16]$ are the most common. Previous research has shown that the oxygen uptake $\left(\mathrm{VO}_{2}\right)$ at the ventilatory anaerobic threshold and at peak exercise $\left(\mathrm{VO}_{\text {2peak }}\right)$ were reduced four weeks after the completion of neoadjuvant chemotherapy in patients with esophageal or gastric cancer and seven weeks after neoadjuvant chemoradiotherapy in patients with rectal cancer, respectively $[15,16]$. Another study has shown that these parameters of aerobic capacity were also reduced immediately after neoadjuvant chemoradiotherapy in patients with rectal cancer [17]. In the same study, a preoperative physical exercise program following neoadjuvant chemoradiotherapy (supervised in-hospital, three training sessions a week) returned the mean decline in $\mathrm{VO}_{\text {2peak }}$ of $2.52 \mathrm{~mL} / \mathrm{kg} / \mathrm{min}$ back to baseline values within six weeks [17]. Hence, neoadjuvant treatment with chemotherapy and radiotherapy reduces aerobic capacity, whereas structured physical exercise training after this treatment is effective in reversing this reduction.

Chemotherapy can also affect skeletal muscle mass. Daly et al. showed significant reductions in cross sectional skeletal muscle area and skeletal muscle mass around the third lumbar vertebrae (L3) in patients with foregut cancer (esophagus, stomach, pancreas, liver, and bile duct) treated with neoadjuvant chemotherapy, with a mean duration between measurements of 118 days [18]. Neoadjuvant chemotherapy is also associated with catabolic loss of skeletal muscle mass and fat over time (mean time between measurements of 86.4 days) in patients with locally advanced gastric cancer [19]. Besides the losses in muscle mass, the latter study found that sarcopenia, defined as; age-related loss of skeletal muscle mass and strength [20], was associated with early termination of the neoadjuvant chemotherapy treatment. Dose-limiting toxicity, meaning dose reduction or termination of the chemotherapy treatment because of toxicity, was observed in $46 \%$ of the patients. In the group of patients with dose-limiting toxicity, there was a higher percentage of sarcopenia ( $80 \%$ vs. $42 \%$; not statistically significant) [19]. Chemotherapy can affect skeletal muscle mass negatively in several patient populations and preservation of skeletal muscle mass is important for continuing and completing the chemotherapy treatment. 


\section{Physical functioning and quality of life}

Treatment for colorectal cancer can also affect physical functioning and quality of life. A study of Cabilan evaluated the short-term impact of curative colorectal cancer treatment (abdominal resection with or without additional (neo)adjuvant treatment) on physical activity, functional status and quality of life. This systematic review showed a decline in physical activity, functional status and quality of life scores up to six months after colorectal cancer treatment [21]. These scores almost returned to baseline levels at one year after treatment. One study in this review investigated factors that influenced physical activity. The results showed that surgical treatment alone was a statistically significant predictor of sufficient physical activity post treatment [22]. Subsequently, it is challenging to achieve the sufficient amount of physical activity for patients that received any additional treatment as chemotherapy or radiotherapy. Results on functional status (measured by self-reported tools) showed that the number of patients functioning independently in activities of daily living in the total patient population decreased post treatment by $12.8 \%$. Hence, colorectal cancer treatment decreases physical activity, functional status and quality of life six months after treatment.

Several other studies evaluated quality of life in long-term colorectal cancer survivors and showed that these people have comparable perceived quality of live compared to matched control subjects [23]. Overall, colorectal cancer treatment can negatively affect physical activity, functional status and quality of life on the short-term. On the long-term, survivors of colorectal cancer are experiencing comparable or higher levels of quality of life compared with matched non-cancer control subjects. Figure 2 shows a schematic hypothesis of the change over time of a patient's functional status during the "journey" of having the first vague signs and symptoms, being diagnosed and treated and recovering from the treatment for colorectal cancer. 


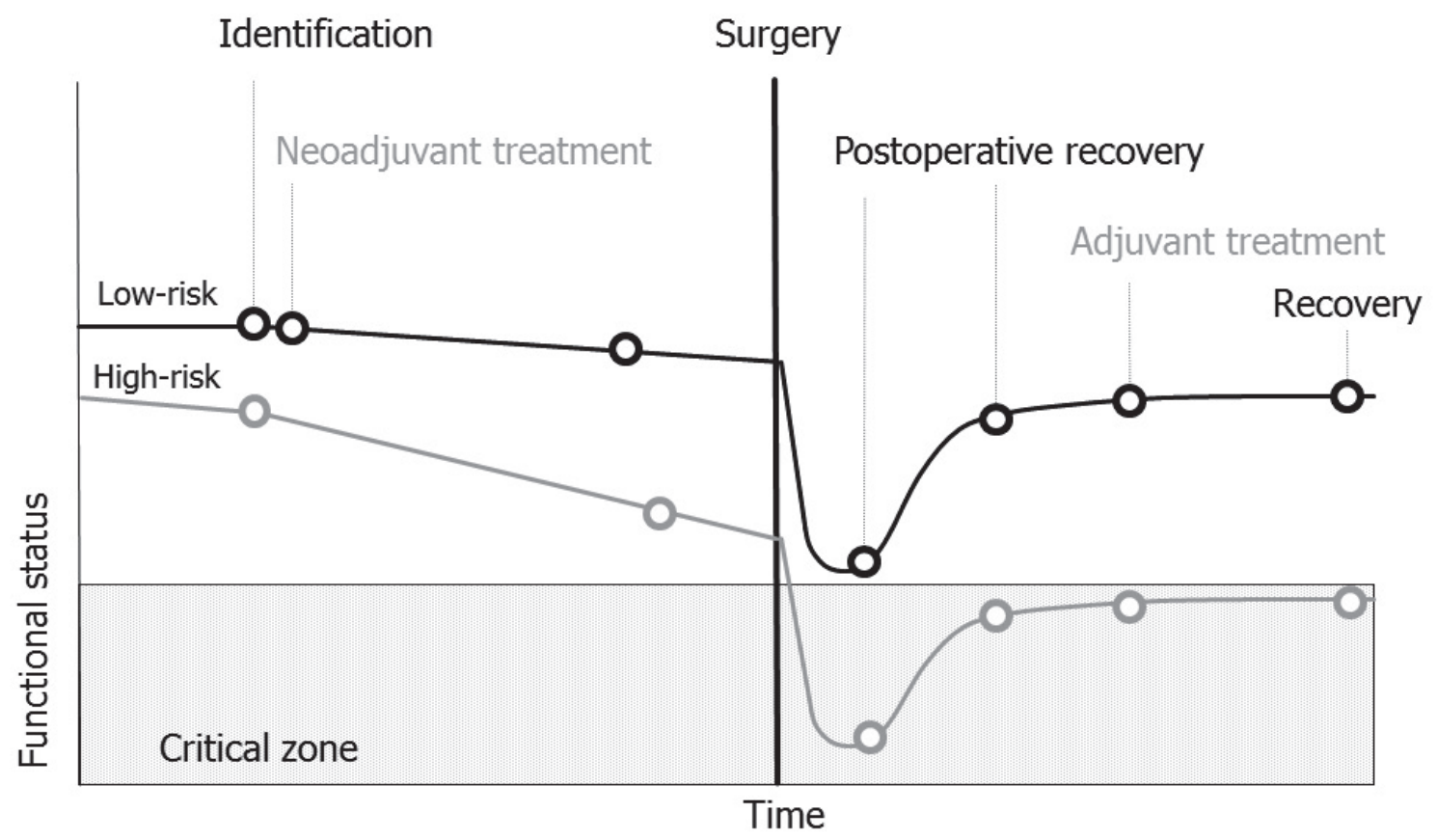

Figure 2. Schematic hypothesis of the change in functional status over time.

\section{TRANSITION OF THE HEALTH CARE SYSTEM}

Demographic shifts and societal changes over the last century challenge health care systems with increasing demands and more specific needs. For example, there is more need for long-term care and chronic disease management [24]. Health care systems have to adapt to meet these needs to stimulate a complete continuum of patient care over time. Consequently, health care systems are undergoing a transformation from a reactive system with the focus on curative medicine to a more proactive approach with predictive, preventive, personalized, and participatory (P4) elements [25]. P4 health is a proactive approach with health care tailored to the specific individual [26].

Prevention of diseases, but also prevention of the impact of a disease, for instance on physical functioning, receives more attention nowadays, and this attention will most likely further increase in the future. An example of early detection in the Netherlands is the national screening program for colorectal cancer. This screening program exists since 2014 (www.bevolkingsonderzoekzuid.nl) in which every citizen between 55 and 75 years of age receives an invitation for this program. With this screening program, it is possible to identify patients with colorectal cancer in an earlier stage of the disease, which increases curative treatment options. Additionally, the program influences the composition of the patient population with colorectal complaints that consult Dutch hospitals. Currently, early preventive screening in the Netherlands already has positive 
effects on the postoperative outcomes of patients with colorectal cancer [8], resulting in lower mortality rates in the group of patients identified via this program $[3,8]$.

Patients with (colorectal) cancer are exposed to specific challenges, which can include loss of body mass, malnutrition, fatigue, anemia, neoadjuvant therapy, surgery, and additional adjuvant therapy. Hereto, coping with this disease becomes a major life event. P4 health with personalized (preventive) interventions [26] can support patients in preserving their level of physical functioning during the treatment course and decrease the patients' experience of disease- and treatment-related negative side effects. Figure 3 shows the innovative journey for colorectal cancer based on the literature explored for the research projects in this thesis.

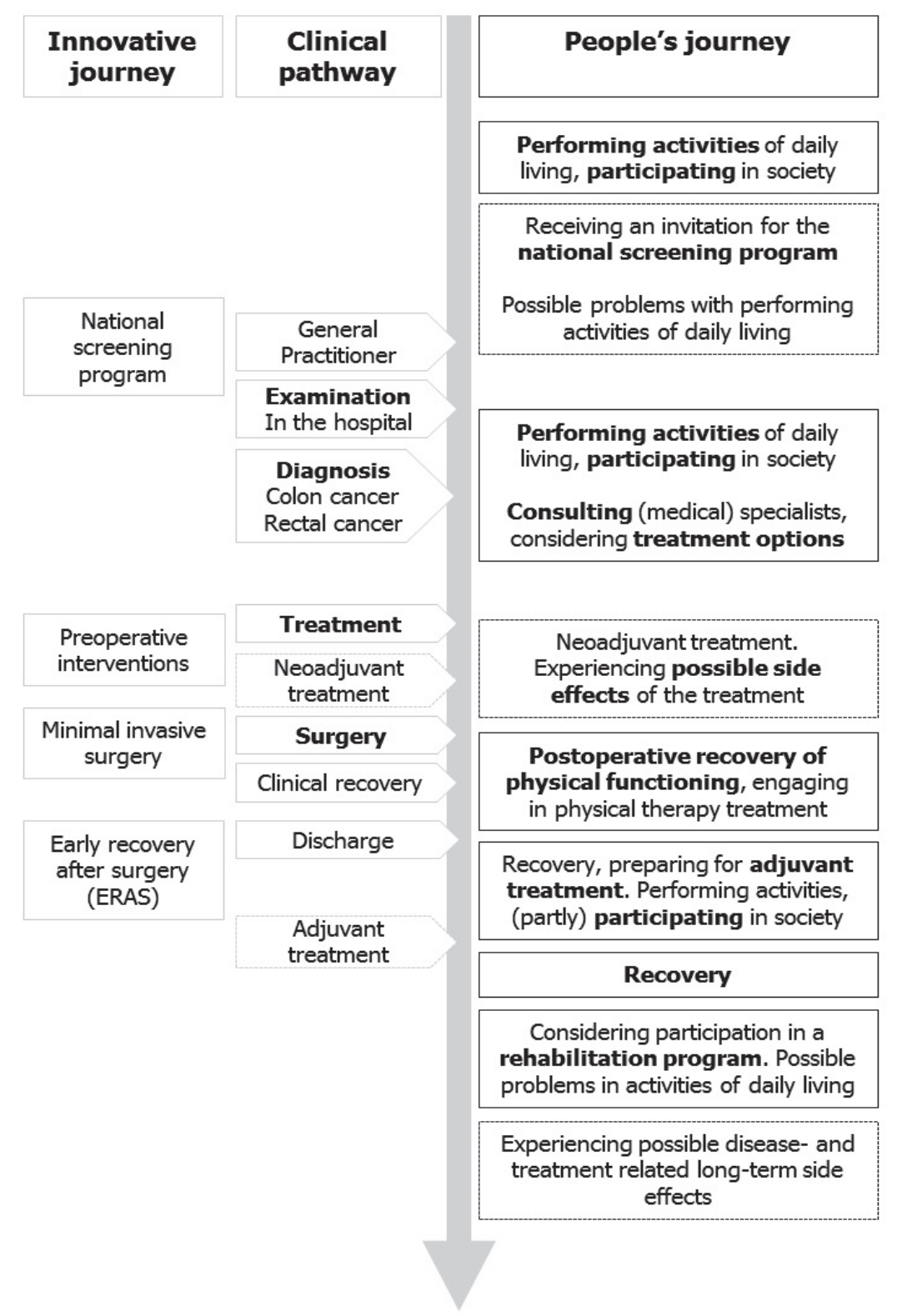

Figure 3. The innovative journey for colorectal cancer. 


\section{OUTCOME AFTER MAJOR ABDOMINAL SURGERY}

Outcome after major abdominal surgery depends on several factors, such as perioperative care, neoadjuvant treatment, and psychophysiological tolerance to surgical stress. Major colorectal surgery is significantly associated with morbidity and mortality $[27,28]$; in approximately $30 \%$ of all surgeries, complications are registered with a mortality rate of 1.1 to $3.0 \%$ [8]. Physical fitness is also an important factor influencing the outcome after major abdominal surgery. Preoperative aerobic capacity, measured during cardiopulmonary exercise testing, has been reported to have a consistent positive relation with postoperative outcomes in major abdominal surgery $[15,29,30]$. A poor aerobic capacity indicates a reduced capacity of the body's oxygen transport and utilization system (physiological reserve capacity), which can contribute to a complicated postoperative time course in patients with lower and upper gastrointestinal cancer, respectively $[15,16]$. The importance of skeletal muscle mass concerning the outcome after major abdominal surgery is also further evaluated. There is an independent association between low skeletal muscle mass and poor overall survival after abdominal resection in patients with advanced rectal cancer [31]. Additionally, sarcopenia is associated with survival, surgical complications, and treatment-related toxicities in patients with colorectal cancer [32, 33] and in general abdominal surgery [34]. Berkel et al. showed that skeletal muscle attenuation was associated with postoperative morbidity in patients with rectal cancer after neoadjuvant treatment and non-laparoscopic rectal resection [35]. The study also found that sarcopenic obesity (defined as body mass index $>25 \mathrm{~kg} / \mathrm{m}^{2}$ and a skeletal muscle mass index below the sex-specific median) was associated with the overall rate of complications after resection for rectal cancer [35]. Therefore, aerobic capacity, skeletal muscle mass, and body composition are important factors that significantly contribute to outcome after major abdominal surgery.

\section{The surgical stress response}

Major surgery induces a stress response, which leads to a reaction of homeostasis and allostasis. Homeostasis is the body's ability to maintain a dynamic internal equilibrium and allostasis means achieving stability through change. Allostasis leads to maintaining homeostasis, which is essential for life [36]. Additionally, major surgery generates a strong systemic inflammatory response that leads to an increase in the oxygen demand (metabolic demand) [37].

Peroperative, the hypothalamic-pituitary-adrenal (HPA) axis and the sympathetic nervous system are activated [38]. Activation of the HPA axis results in the release of glucocorticoids. Activation of the sympathetic nervous system leads to an increase in hormone release (cortisol, glucagon, catecholamines, and inflammatory cytokines) [39]. These hormonal changes lead to an increased catabolism of stored energy $[39,40]$. Protein catabolism results in a marked loss of body mass and skeletal muscle waste. The 
stress response caused by surgery leads also to an increased oxygen demand and major abdominal surgery is associated with an increase of oxygen demand of $40 \%$ or more [41]. This increase must be met by increases in cardiac output and tissue oxygen extraction $[37,41]$. The magnitude and duration of the surgical stress response are linked to the surgical injury caused by the type of the surgical procedure and the development of complications [34]. Additionally, the increased metabolic and catabolic responses can lead to a delayed recovery with a decline in physical functioning and the ability to perform activities of daily living. Hence, aerobic capacity and muscular reserves should be sufficient in patients undergoing major abdominal surgery. Patients with a higher preoperative level of aerobic capacity may have a greater physiological reserve to cope with the surgical stress response $[30,42]$.

reservation of aerobic capacity and skeletal muscle mass is also important for maintaining and continuing the additional medical treatment after elective surgery (e.g., adjuvant chemotherapy and/or radiotherapy) and the recovery from this adjuvant treatment [19]. Additionally, this capacity can be of use in maintaining chemotherapy treatment with the proposed dose. Consequently, an optimal level of aerobic capacity and skeletal muscle mass can support patients during the adjuvant medical treatment.

\section{PHYSICAL THERAPY MANAGEMENT}

Physical therapy mainly focuses on physical functioning and physical abilities. This includes helping people maintain, recover, or improve their functional and physical abilities in order to preserve their participation in society as long and as optimal as possible. These abilities can be impaired due to a (medical) condition, an injury, or due to hospitalization and/or surgery. In major abdominal surgery, patients can be guided by the physical therapist in improving their physical abilities during the postoperative inhospital period and the period after discharge. Postoperative physical therapy treatment often consists of airway clearing exercises, resistance exercises, practicing ambulation, walking, stair climbing, and improving skeletal muscle function and aerobic capacity. Early postoperative mobilization with different exercises (aerobic training and resistance training) improves the patient's physical functioning after major abdominal cancer surgery [43]. Physical therapy after discharge can be performed in an outpatient clinic, a community-based facility or in the patient's home context. The physical therapy is built around the patient's individual goals, needs and capabilities.

\section{Prehabilitation}

In the time frame between the (cancer) diagnosis and the definitive surgical procedure, there is a window of opportunity to optimize physical functioning (e.g., aerobic capacity, skeletal muscle mass). Especially for patients with cancer it is important to maintain or 


\section{Chapter 1}

improve physical functioning during the complete treatment course, as they are often faced with the double challenge of surgery and additional cancer treatment, such as (neo)adjuvant chemotherapy. Prehabilitation can be used to anticipate on an upcoming stressor as elective surgery [44], and can be defined as "a process in the continuum of care that occurs between the time of (cancer) diagnosis and the beginning of acute treatment and includes physical and psychological assessments that establish a baseline functional level, identify impairments, and provide interventions that promote physical and psychological health to reduce the incidence and/or severity of future impairments" [45]. Prehabilitation is based on the principle that structured preoperative physical exercise training, nowadays often combined with nutritional support, provides patients with a psychophysiological buffer to better withstand the stress of surgery [46-49]. A review of West et al. showed that physical exercise training before surgery is safe and feasible [50]. Furthermore, it is important to create a proactive culture through the whole continuum of care in order to keep all patients physically fit and active during the treatment and disease course [44].

\section{Preoperative risk management stratification}

Before starting preoperative interventions (prehabilitation), there should be an adequate risk stratification procedure to identify patients at risk for a complicated postoperative period at an early stage [44]. By doing this, vulnerable high-risk patients can be distinguished from low-risk patients, and care strategies can be tailored to each patient [51]. Accurate preoperative identification of (high-risk) patients can help the patient and health care professionals to make appropriate decisions about undergoing surgery or substitute treatments, and for example about the need for additional preoperative interventions. Cardiopulmonary exercise testing can be used as a preoperative risk stratification tool for patients undergoing non-cardiopulmonary intra-abdominal surgery $[48,49]$. This test is the gold standard test to assess a patient's aerobic capacity [51] and can support clinical decision-making [52]. Research addressing the usefulness of more practical performance-based tests that estimate aerobic capacity for preoperative risk stratification, such as the incremental shuttle walk test, stair-climbing test, and 6-minute walk test is increasing [53-56]. Figure 4 shows a schematic hypothesis of the change in functional status during treatment with the addition of prehabilitation. 


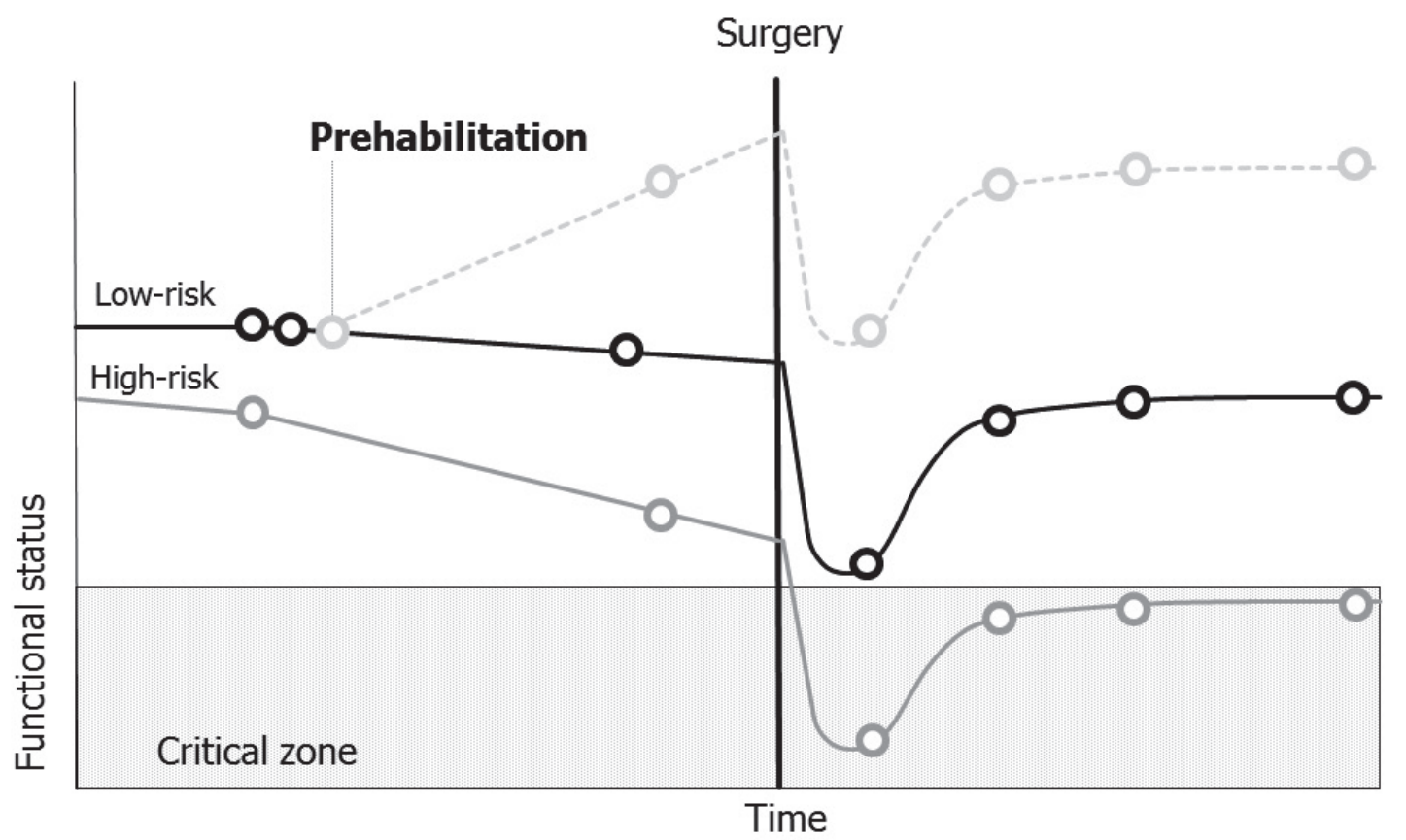

Figure 4. Schematic hypothesis of the change in functional status over time with the addition of prehabilitation.

\section{POSTOPERATIVE RISK ASSESSMENT}

Minimal invasive surgery decreases the stress response and thereby the surgical impact. Furthermore, minimal invasive surgery offers patients several benefits as smaller incisions, decreases in blood loss, and a reduction in pain and scarring. Together with enhanced recovery programs, minimal invasive surgery can positively contribute to the postoperative recovery of physical functioning of patients undergoing major abdominal surgery. Furthermore, it is important for patients to be physically active during the hospital stay and to avoid bed rest. Previous studies showed that $80 \%$ of a general hospital day is spend in bed [57], whereas bed rest leads to catabolic changes in skeletal muscles and bones induced by non-use [58]. Changes in skeletal muscle mass and quality occur, which can lead to loss of muscle strength and function [58]. Consequently, the patient's physical functional abilities will decrease.

In a large proportion of the elderly patients, the capacity to perform activities of daily living decreases during a hospital stay [59]. A recent study showed that hospitalization in the year before oncologic resection is associated with an increased risk for postoperative adverse events (complications, complex discharge, 90-day readmission) [60]. These findings emphasize the negative effects of hospitalization, bed rest, and the additional risks for recovery of physical functioning, when a patient is admitted to the hospital. Hospitalized patients should be stimulated by health care professionals to be physically active during the hospital stay, with attention for fast-track mobilization in the 


\section{Chapter 1}

postoperative phase, and for an active culture and infrastructure [44]. Besides the aspect of physical activity, it is important for patients to rest and sleep during hospitalization, but there should be a natural balance between sleeping and being active. Better postoperative sleep can stimulate postoperative recovery, as it decrease postoperative pain and fatigue [61], which again can influence postoperative physical activity and stimulate postoperative recovery of physical functioning.

\section{AIM AND OUTLINE OF THIS THESIS}

The main aim of this thesis was to monitor and evaluate patient characteristics, physical functioning, preoperative physical exercise training (prehabilitation) and postoperative outcomes in patients with colorectal cancer scheduled for elective abdominal resection. In order to investigate the entire people's journey and treatment course and, based hereupon, to develop and implement optimal evidence-based physical therapy management during the pre- and postoperative care pathway for these patients.

Chapter 2 describes the content and between-hospital variation in preoperative and postoperative physical therapist management in patients who opt for major elective abdominal surgery for colorectal, hepatic, or pancreatic cancer in the Netherlands.

- In chapter 3, the associations between preoperative performance parameters of physical fitness and postoperative outcomes in patients with colorectal cancer scheduled for elective colorectal resection are evaluated.

- Chapter 4 evaluates the changes in performance-based physical fitness and computed tomography-derived skeletal muscle measurements, before and after neoadjuvant chemoradiotherapy in individual patients with locally advanced rectal cancer scheduled for elective abdominal resection.

- In chapter 5, the feasibility and preliminary effectiveness of a supervised outpatient physical exercise training program during neoadjuvant chemoradiotherapy in individual patients with rectal cancer is described.

- Chapter 6 describes the study protocol of a one-group pre-test post-test pilot study to evaluate the feasibility of and individual responses to a home-based prehabilitation program for high-risk patients undergoing major elective abdominal surgery.

- Finally, chapter 7 provides the general discussion of this thesis. In this final chapter, the main findings and limitations of the studies in this thesis will be discussed. 


\section{REFERENCES}

1. World Health organization, Men, ageing and health: achieving health across the life span. https://www.who.int/ageing/publications/men/en/, 2019 (accessed January 2019).

2. World cancer research fund international, Global cancer statistics for the most common cancers. https://www.wcrf.org/dietandcancer/cancer-trends/worldwide-cancer-data, 2019 (accessed January 2019).

3. de Neree Tot Babberich MP, Vermeer NC, Wouters MW, et al., Postoperative outcomes of screendetected vs non-screen-detected colorectal cancer in the Netherlands, JAMA Surg. 153 (2018) e183567.

4. Gustafsson UO, Hausel J, Thorell A, et al., Adherence to the enhanced recovery after surgery protocol and outcomes after colorectal cancer surgery, Arch Surg. 146 (2011) 571-577.

5. Slim K, Fast-track surgery: the next revolution in surgical care following laparoscopy, Colorectal Dis. 13 (2011) 478-480.

6. Greer NL, Gunnar WP, Dahm P, et al., Enhanced recovery protocols for adults undergoing colorectal surgery: a systematic review and meta-analysis, Dis Colon Rectum. 61 (2018) 1108-1118.

7. Greco M, Capretti G, Beretta L, et al., Enhanced recovery program in colorectal surgery: a metaanalysis of randomized controlled trials, World J Surg. 38 (2014) 1531-1541.

8. Dutch Institute for Clinical Auditing (DICA) Leiden, DICA Jaarrapportage 2017: DCRA. https://dica.nl/jaarrapportage-2017/dcra, 2019 (accessed January 2019).

9. Bruins, BJ, Brief van de minister voor medische zorg, Tweede kamer, vergaderjaar 2017-2018, 32 793, 301 (2018).

10. The Netherlands Comprehensive Cancer Organisation (IKNL), Cijfers over kanker, The Netherlands 2018. https://www.cijfersoverkanker.nl/selecties/Dataset_1/ img5d09faff4c555, 2019 (accessed January 2019).

11. Chau I, Brown G, Cunningham D, et al., Neoadjuvant capecitabine and oxaliplatin followed by synchronous chemoradiation and total mesorectal excision in magnetic resonance imaging-defined poor-risk rectal cancer, J Clin Oncol. 24 (2006) 668-674.

12. Ludmir EB, Palta M, Willett CG, et al., Total neoadjuvant therapy for rectal cancer: An emerging option, Cancer. 123 (2017) 1497-1506.

13. Swellengrebel HA, Marijnen CA, Verwaal VJ, et al., Toxicity and complications of preoperative chemoradiotherapy for locally advanced rectal cancer, Br J Surg. 98 (2011) 418-426.

14. Windsor PM, Potter J, McAdam K, et al., Evaluation of a fatigue initiative: information on exercise for patients receiving cancer treatment, Clin Oncol (R Coll Radiol) 21 (2009) 473-482.

15. West MA, Loughney $L$, Barben $C P$, et al., The effects of neoadjuvant chemoradiotherapy on physical fitness and morbidity in rectal cancer surgery patients, Eur J Surg Oncol. 40 (2014) 1421-1428.

16. Jack $S$, West MA, Raw $D$, et al., The effect of neoadjuvant chemotherapy on physical fitness and survival in patients undergoing oesophagogastric cancer surgery, Eur J Surg Oncol 40 (2014) 13131320.

17. West MA, Loughney L, Lythgoe $D$, et al., Effect of prehabilitation on objectively measured physical fitness after neoadjuvant treatment in preoperative rectal cancer patients: a blinded interventional pilot study, Br J Anaesth. 114 (2015) 244-251.

18. Daly LE, Ní Bhuachalla ÉB, Power DG, et al., Loss of skeletal muscle during systemic chemotherapy is prognostic of poor survival in patients with foregut cancer, J Cachexia Sarcopenia Muscle. 9 (2018) 315-325.

19. Palmela C, Velho S, Agpstomjp L, et al., Body composition as a prognostic factor of neoadjuvant chemotherapy toxicity and outcome in patients with locally advanced gastric cancer, J Gastric cancer. 17 (2017) 74-87. 


\section{Chapter 1}

20. Cruz-Jentoft AJ, Bahat G, Bauer J, et al., Sarcopenia: revised European consensus on definition and diagnosis, Age Ageing. 48 (2019) 16-31.

21. Cabilan $\mathrm{CJ}$, Hines $\mathrm{S}$, The short-term impact of colorectal cancer treatment on physical activity, functional status and quality of life: a systematic review, JBI Database System Rev Implement Rep. 15 (2017) 517-566.

22. Lynch BM, Cerin E, Newman B, et al., Physical activity, activity change, and their correlates in a population-based sample of colorectal cancer survivors, Ann Behav Med. 34 (2007) 135-143.

23. Hart TL, Charles ST, Gunaratne M, et al., Symptom severity and quality of life among long-term colorectal cancer survivors compared with matched control subjects: a population-based study, Dis Colon Rectum. 61 (2018) 355-363.

24. Heerkens $\mathrm{YF}$, de Weerd $\mathrm{M}$, Huber $\mathrm{M}$, et al., Reconsideration of the scheme of the international classification of functioning, disability and health: incentives from the Netherlands for a global debate, Disabil Rehabil. 40 (2018) 603-611.

25. Flores M, Glusman G, Brogaard K, et al., P4 medicine: how systems medicine will transform the healthcare sector and society, Per Med. 10 (2013) 565-576.

26. Hood $L$, Flores $M, A$ personal view on systems medicine and the emergence of proactive $P 4$ medicine: predictive, preventive, personalized and participatory, N Biotechnol. 29 (2012) 613-624.

27. Andreoni B, Chiappa A, Bertani E, et al., Surgical outcomes for colon and rectal cancer over a decade: results from a consecutive monocentric experience in 902 unselected patients, World J Surg Oncol. 5 (2007) 73.

28. Burns EM, Bottle A, Aylin $\mathrm{P}$, et al., Variation in reoperation after colorectal surgery in England as an indicator of surgical performance: retrospective analysis of Hospital Episode Statistics, BMJ. 16 (2011) 343.

29. Snowden CP, Prentis J, Jacques B, et al., Cardiorespiratory fitness predicts mortality and hospital length of stay after major elective surgery in older people, Ann Surg. 257 (2013) 999-1004.

30. Levett DZ, Grocott MP, Cardiopulmonary exercise testing for risk prediction in major abdominal surgery, Anesthesiol Clin. 33 (2015) 1-16.

31. Takeda Y, Akiyoshi T, Matsueda K, et al., Skeletal muscle loss is an independent negative prognostic factor in patients with advanced lower rectal cancer treated with neoadjuvant chemoradiotherapy, PLoS One. 13 (2018) e0195406.

32. Boer BC, de Graaff F, Brusse-Keizer M, et al., Skeletal muscle mass and quality as risk factors for postoperative outcome after open colon resection for cancer, Int J Colorectal Dis. 31 (2016) 1117-1124.

33. van Vledder MG, Levolger $\mathrm{S}$, Ayez $\mathrm{N}$, et al., Body composition and outcome in patients undergoing resection of colorectal liver metastases, Br J Surg. 99 (2012) 550-557.

34. Jones K, Gordon-Weeks A, Coleman C, et al., Radiologically determined sarcopenia predicts morbidity and mortality following abdominal surgery: a systematic review and meta-analysis, World J Surg. 41 (2017) 2266-2279.

35. Berkel AEM, Klaase JM, de Graaff F, et al., Patient's skeletal muscle radiation attenuation and sarcopenic obesity are associated with postoperative morbidity after neoadjuvant chemoradiation and resection for rectal cancer, Dig Surg. 13 (2018) 1-8.

36. McEwen BS, Wingfield JC. What is in a name? Integrating homeostasis, allostasis and stress, Horm Behav. 57 (2010) 105-11.

37. Davies SJ, Wilson RJ, Preoperative optimization of the high-risk surgical patient, $\mathrm{Br}$ J Anaesth. 93 (2004) 121-128.

38. Webster Marketon JI, Glaser R. Stress hormones and immune function, Cell Immunol. 252 (2008) 16-26.

39. Finnerty CC, Mabvuure NT, Ali A, et al., The surgically induced stress response, JPEN J Parenter Enteral Nutr. 37 (2013) 21S-9S. 
40. Desborough JP, The stress response to trauma and surgery, $\mathrm{Br}$ J Anaesth. 85 (2000) 109-117.

41. Older $P$, Hall $A$, Clinical review: how to identify high-risk surgical patients, Crit Care. 8 (2004) 369-372.

42. Bongers $B C$, Berkel $A E$, Klaase JM, et al., An evaluation of the validity of the pre-operative oxygen uptake efficiency slope as an indicator of cardiorespiratory fitness in elderly patients scheduled for major colorectal surgery, Anaesthesia. 72 (2017) 1206-1216.

43. de Almeida EP, de Almeida JP, Landoni G, et al., Early mobilization programme improves functional capacity after major abdominal cancer surgery: a randomized controlled trial, $\mathrm{Br}$ J Anaesth. 119 (2017) 900-907.

44. Hulzebos EH, van Meeteren NL, Making the elderly fit for surgery, Br J Surg. 103 (2016) e12-15.

45. Silver JK, Baima J, Cancer prehabilitation: an opportunity to decrease treatment-related morbidity, increase cancer treatment options, and improve physical and psychological health outcomes, Am J Phys Med Rehabil. 92 (2013) 715-727.

46. Moorthy K, Wynter-Blyth V, Prehabilitation in perioperative care, Br J Surg. 104 (2017) 802-803.

47. van Rooijen SJ, Engelen MA, Scheede-Bergdahl C, et al., Systematic review of exercise training in colorectal cancer patients during treatment, Scand J Med Sci Sports. 28 (2018) 360-370.

48. Minnella EM, Bousquet-Dion G, Awasthi R, et al., Multimodal prehabilitation improves functional capacity before and after colorectal surgery for cancer: a five-year research experience, Acta Oncol. 56 (2017) 295-300.

49. Carli F, Charlebois P, Stein B, et al., Randomized clinical trial of prehabilitation in colorectal surgery, Br J Surg. 97 (2010) 1187-1197.

50. West MA, Wischmeyer $\mathrm{PE}$, Grocott MP, Prehabilitation and nutritional support to improve perioperative outcomes, Curr Anesthesiol Rep. 7 (2017) 340-349.

51. Older PO, Levett DZ, Cardiopulmonary exercise testing and surgery, Ann Am Thorac Soc. 14 (2017) S74-S83.

52. Moran J, Wilson F, Guinan E, et al., The preoperative use of field tests of exercise tolerance to predict postoperative outcome in intra-abdominal surgery: a systematic review, J Clin Anesth. 35 (2016) 446-455.

53. Nutt CL, Russell JC, Use of the pre-operative shuttle walk test to predict morbidity and mortality after elective major colorectal surgery, Anaesthesia. 67 (2012) 839-849.

54. Struthers R, Erasmus $P$, Holmes $K$, et al., Assessing fitness for surgery: a comparison of questionnaire, incremental shuttle walk, and cardiopulmonary exercise testing in general surgical patients, Br J Anaesth. 101 (2008) 774-780.

55. Reddy S, Contreras CM, Singletary B, et al., Timed stair climbing is the single strongest predictor of perioperative complications in patients undergoing abdominal surgery, J Am Coll Surg. 222 (2016) 559-566.

56. Lee $\mathrm{CH}$, Kong JC, Ismail $\mathrm{H}$, et al., Systematic review and meta-analysis of objective assessment of physical fitness in patients undergoing colorectal cancer surgery, Dis Colon Rectum. 61 (2018) 400-409.

57. Brown $\mathrm{CJ}$, Redden $\mathrm{DT}$, Flood $\mathrm{KL}$, et al., The underrecognized epidemic of low mobility during hospitalization of older adults, J Am Geriatr Soc. 57 (2009) 1660-1665.

58. Bloomfield SA, Changes in musculoskeletal structure and function with prolonged bed rest, Med Sci Sports Exerc. 29 (1997) 197-206.

59. Covinsky KE, Palmer RM, Fortinsky RH, et al., Loss of independence in activities of daily living in older adults hospitalized with medical illnesses: increased vulnerability with age, J Am Geriatr Soc. 51 (2003) 451-458.

60. Sharoky CE, Collier KT, Wirtalla CJ, et al., Hospitalization in the year preceding major oncologic surgery increases risk for adverse postoperative events, Ann Surg Oncol. 24 (2017) 3477-3485.

61. Su X, Wang DX. Improve postoperative sleep: what can we do? Curr Opin Anaesthesiol. 31 (2018) 83-88. 



\section{Chapter}

\section{Variation in preoperative and postoperative physical therapist management for patients opting for elective abdominal surgery}

Aniek F. Heldens *

Christel A. van Beijsterveld *

Bart C. Bongers

Nico L. van Meeteren

* Shared first authorship

Physical Therapy 2019 (in press). 


\title{
Chapter 2
}

\begin{abstract}
Background.

Evidence about the role of physical therapy in perioperative care pathways to improve postoperative outcomes is growing. However, it is unclear whether research findings have been translated in daily practice. The objectives of this study were to describe the current content and between-hospital variability of perioperative physical therapist managament for patients undergoing colorectal, hepatic, or pancreatic resection in the Netherlands and to compare the currently recommended state-of-the-art physical therapy with the self-reported daily clinical physical therapist management.
\end{abstract}

\section{Methods.}

Hospital physical therapists were asked to complete an online survey about pre- and postoperative physical therapist management at their hospital. To explore variability of perioperative physical therapist management between the hospitals, frequency variables were clustered to determine the level of uniformity. Latent class analysis (LCA) was performed to identify clusters of hospitals with certain homogeneous characteristics on a 19-item dichotomous scale.

\section{Results.}

Of 82 eligible Dutch hospitals, 65 filled out the survey (79.3\%). Preoperative physical therapy was performed in 34 hospitals (54.0\%; 2/65 responding hospitals were excluded from the data analysis). Postoperative physical therapy was performed in all respondents, focusing mainly on regaining independent physical functioning. LCA identified a threeclass model. Hospitals in class I and II were more likely to provide preoperative physical therapist interventions compared to class III.

\section{Conclusions.}

There is a wide degree of variability between hospitals regarding pre- and postoperative physical therapist practice for patients opting for major abdominal surgery. Three different classes of daily practice were identified. Further translation of key research findings into daily physical therapist practice is advised, especially for hospitals in which the physical therapist is not involved preoperatively. Moreover, improving uniformity by developing up-to-date clinical guidelines is recommended. 


\section{INTRODUCTION}

According to the World Health Organization, the number of people aged 65 and older is expected to increase to 1.5 billion in 2050 , representing $16.0 \%$ of the world's population [1]. With the aging population, the number of elderly requiring surgical procedures is increasing. Elderly patients may experience difficulties when recovering from surgery due to diminished physiological reserves, frailty, and comorbidities [2-4]. In the last decades, beneficial improvements have therefore been made in perioperative care management. Developments in analgesic approaches and surgical techniques, as well as "enhanced recovery after surgery" (ERAS) and "fast track" programs resulted in a reduced length of hospital stay and a reduction in overall complications [5].

However, the stress response to surgery and hospitalization comprises metabolic and physiological changes and results in an increase in allostatic load $[6,7]$. A vulnerable patient with comorbid conditions and reduced physiological reserves opting for major elective surgery may have less capacity to adapt to the increased allostatic load. This may lead to an imbalance in autonomic, endocrine, metabolic, and immune function, resulting in additional clinical challenges leading to a delayed recovery of physical functioning [8], or even a permanent loss of physical functioning.

Hereto, a change in the continuum of care is advocated with complementary prevention and care interventions to assist the vulnerable patient in managing their perioperative course [9-11]. This implies a changing role for the physical therapist in acute care settings concerning patient care and professionalism, as already mentioned by Lopopolo et al. in 1999 [12]. Improvements in perioperative care should best include innovative physical therapist interventions to prevent a complicated peri- and postoperative course, as well as to enhance a rapid return to adequate performance of activities of daily living that are essential to preserve independent physical functioning and perceived quality of life. Innovative pre- and postoperative physical therapist interventions should lead towards realization of predictive, preventive, personalized, and participatory (P4) perioperative physical therapy [13]. The latter means including preoperative risk stratification using performance-based tests, preoperative advice and recommendations about the importance of physical activity and physical fitness, preoperative exercise training (prehabilitation) for high-risk patients, and early mobilization and functional physical exercise training postoperatively, as recommended in several guidelines $[9,10,14-17]$. High-quality research demonstrates that preoperative physical therapist interventions for high-risk patients undergoing cardiac, abdominal, and major joint replacement surgery are feasible and effective [18-22].

It remains unclear to what degree these research findings already have been translated and implemented in the real-life context of health care systems, especially in the routines of physical therapist services. Consequently, it is necessary to establish an understanding 


\section{Chapter 2}

of the current clinical practice in order to compare the differences between clinical research outcomes and clinical practice, as well as to anticipate on what should be done to change clinical practice and education for physical therapist students according to the latest evidence. Nationwide surveys have been performed to explore the clinical physical therapist management in different fields to examine practice variability with respect to known evidence and guidelines [23-27]. Benefits of such an approach were shown previously by Peter et al. [27] with improvements of guideline recommendations for postacute physical therapist practice after major joint replacement. To provide optimal physical therapist care for patients opting for major abdominal surgery this approach might be beneficial as well. Therefore, the aims of the present study were two-fold: to describe the overall reported content and between-hospital variability of perioperative physical therapist management in patients who opt for major elective abdominal surgery for colorectal, hepatic, or pancreatic cancer in the Netherlands, and to compare the nowadays-advised state-of-the-art physical therapy with the self-reported daily clinical physical therapist management. With state-of-the-art physical therapist management, we refer to the current highest level of scientific evidence available in perioperative physical therapist management.

\section{METHODS}

\section{Design}

In this cross-sectional survey study, all heads of the departments of physical therapy who were registered with the Dutch Association for Physical therapy in Hospitals (NVZF) and the Association of Intramural Physical therapy Managers (VLF) were contacted in November 2016 by e-mail. They were informed about the purpose of the study and the content of the survey. Furthermore, they were asked to forward a participant information letter with the survey to the physical therapist at their department with the most expertise in working with patients undergoing major elective abdominal surgery for colorectal, hepatic, or pancreatic cancer. An e-mail reminder was sent each month for three months to the heads of the departments of physical therapy of which no response was yet obtained. Additionally, two short news items were published in the monthly newsletters of both the NVZF and VLF that described the study and explained how to participate. One news item was published at the beginning of the study and one after three months. Hospitals that not yet filled out the survey after three months were contacted by phone. 


\section{Ethics}

The participant information letter clearly stated that only coded survey data will be used for publication. Because of the voluntary nature of the survey without patient involvement, the study does not meet the criteria for the Dutch medical research involving human subjects act (WMO). Therefore, assessment by a medical ethical review committee was not necessary. None of the respondents objected and a full response on the survey was interpreted as informed consent.

The survey consisted of 41 mixed questions (six open and 35 multiple choice) in the following four domains: 1) demographic data (five questions), 2) preoperative diagnostics and treatment data (twelve questions), 3) postoperative diagnostics and treatment data (seventeen questions), and 4) discharge and readmission data (seven questions). The survey was mainly based on a survey study with a similar research aim [26]. The current survey was shaped with concepts of the framework of Hulzebos \& van Meeteren $[9,10]$ and a literature review. This to be able to inquire whether hospitals provide the nowadays-advised state-of-the-art pre- and postoperative physical therapist management in patients scheduled for major elective abdominal surgery. Additionally, the survey included six questions concerning the context of the postoperative physical therapist treatment (two items) and the availability and usefulness of a readmission protocol (four items). The final version of the survey was peer-reviewed by three (hospital) physical therapists with experience and knowledge about the perioperative care pathway at the Maastricht University Medical Center. The survey was tested in a semistructured pilot process using written and verbal feedback of the assessors. The survey was evaluated on the number and type of questions, relevance of each question, wording, and whether the survey questions captured the total perioperative care pathway. The survey is available as supplementary file (translated into English, not an official cross-cultural adaptation). The survey was developed and administered using Qualtrics electronic survey software (www.qualtrics.com) provided by Maastricht University, Maastricht, the Netherlands. This manuscript is reported according to the STROBE guideline for cross-sectional studies.

\section{Participants}

Physical therapists employed in an acute-hospital care setting in the Netherlands were inquired by a survey about the preoperative and postoperative physical therapist management at their hospital in patients undergoing elective colorectal, hepatic, or pancreatic resection. Hospitals not performing these types of surgery were excluded. 


\section{Data analysis}

Two researchers (C.A.B and A.F.H) categorized the answers of the open questions. When differences between assessors were found, a third researcher (B.C.B) was involved to obtain consensus. The Statistical Package for the Social Sciences for Windows (version 23.0; IBM, SPSS Inc., Chicago, IL, USA) was used for the descriptive data analysis, which was used to describe the study population and responses to the survey. To explore the variability of the perioperative physical therapist management between the hospitals, frequency variables were clustered to determine the level of uniformity, presented as the percentage of respondents choosing the same answer. The categories of uniformity were classified as follows: 1$)$ no uniformity $(<60.0 \%), 2)$ low uniformity $(61.0 \%-70.0 \%), 3)$ moderate uniformity $(71.0 \%-80.0 \%)$, 4) strong uniformity $(81.0 \%-90.0 \%)$, and 5$)$ very strong uniformity $(91.0 \%-100.0 \%)$.

To compare the nowadays-advised state-of-the-art physical therapy with the selfreported daily clinical physical therapist practice, several items from each subdomain of the survey that are closely related to the described conceptual hypothesis in the literature for perioperative physical therapist management were extracted. These items were independently extracted from the survey by three individual assessors (C.A.B, A.F.H, and B.C.B) and resulted in a 19-item dichotomous scale containing seven items on preoperative diagnostics and treatment, eight items addressing postoperative diagnostics and treatments, and four items concerning discharge and readmission (the items are listed in Table 4). Based on the reported clinical physical therapist management, each hospital was subsequently scored on the 19-item dichotomous scale.

Exploratory latent class analysis (LCA) was used to identify and classify clusters of hospitals with certain homogeneous characteristics on the 19-items dichotomous scale. Selection of the optimal number of classes was based on several goodness-of-fit parameters. The following statistical fit-indices were used in this study: the Akaike information criterion (AIC) and the Bayesian information criteria (BIC). Lower AIC and BIC values indicate a better fit. Furthermore, selection of the classes were based on their substantive meaningfulness (the classes should be distinct and meaningful for the clinical expert). LCA was performed with the open source statistical package R (version 2.14.2, R Foundation for Statistical Computing, Vienna, Austria) using the package poLCA [28] version 1.4. In addition, an index (frequency distribution) score was calculated based on the given response by the hospitals on all items at the 19-item dichotomous scale. These scores represent the distance between the nowadays-advised state-of-the-art physical therapy and the self-reported daily clinical physical therapist management. Respondents with scores above $75.0 \%$ were classified as "progressive", respondents with scores between $50.0 \%$ and $75.0 \%$ were classified as "moderately progressive", and respondents with scores lower than $50.0 \%$ were classified as "conservative". 


\section{RESULTS}

\section{Flow of participants through the study}

Of the 103 Dutch hospitals, 21 hospitals (20.4\%) were excluded on beforehand, as they did not perform colorectal, hepatic, or pancreatic resection. The remaining 82 hospitals $(82 / 103,79.6 \%)$ were contacted for participation in the survey. After an inclusion period of three months, 65 of these 82 eligible hospitals responded to the survey, resulting in a response rate of $79.3 \%$. Two responding hospitals $(2 / 65,3.1 \%)$ were excluded from data analysis because they did not meet the inclusion criteria for performing elective colorectal, hepatic, or pancreatic resection. Hence, a total of 63 surveys $(63 / 82,76.8 \%)$ were included for data analysis. Figure 1 shows the flowchart of the study. Demographic and descriptive data concerning the reported pre- and postoperative clinical physical therapist management from the responding hospitals are provided in Table 1, 2a and $2 b$. 


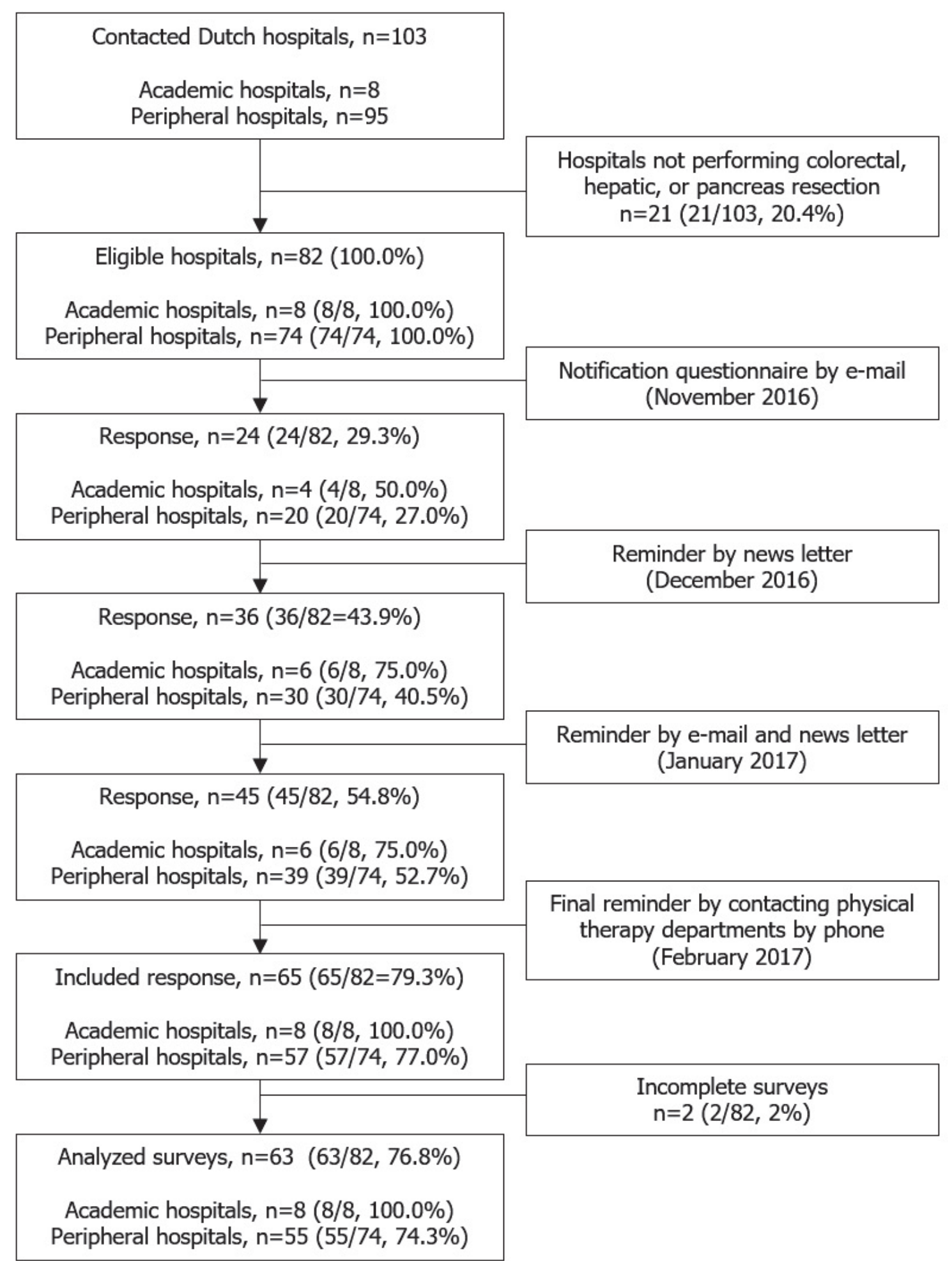

Figure 1. Flowchart of the study. 
Table 1. Demographic data of the responding hospitals $(n=63)$.

\begin{tabular}{|l|c|c|}
\hline Demographic & $\begin{array}{c}\text { No. (\%) of } \\
\text { Hospitals }\end{array}$ & $\begin{array}{c}\text { Mean (SD) \% } \\
\text { of therapists }\end{array}$ \\
\hline Type of hospital & $8(12.7)$ & \\
\hline Academic & $52(82.5)$ & \\
\hline General & $3(4.8)$ & \\
\hline Other & & $76.3(7.4)$ \\
\hline Education level of hospital physical therapist & & $16.2(3.3)$ \\
\hline Bachelor of Applied Science degree & & $6.6(2.1)$ \\
\hline Clinical specialization in physical therapy & & \\
\hline Master of Science degree & & \\
\hline Doctor of Philosophy degree & & \\
\hline Standardized care pathway for major abdominal surgery & \\
\hline Yes & $43(68.3)$ & \\
\hline Physical therapist involved in the development & $31(72.1)$ & \\
\hline No & $20(31.7)$ & \\
\hline Type of standardized care pathway (n=43) ${ }^{\text {a }}$ & & \\
\hline Enhanced recovery after surgery & $27(62.8)$ & \\
\hline Fast-track & $10(23.3)$ & \\
\hline Other & $8(18.6)$ & \\
\hline Protocol-guided physical therapy & $49(77.8)$ & \\
\hline Yes & $14(22.2)$ & \\
\hline No & \\
\hline
\end{tabular}

a Multiple answers possible. 


\section{Chapter 2}

Table 2a. Preoperative clinical physical therapist management ${ }^{\mathrm{a}}$ for patients who underwent elective colorectal, hepatic, or pancreatic resection.

\begin{tabular}{|c|c|}
\hline Physical therapist management & $\begin{array}{l}\text { No. }(\%) \text { of } \\
\text { Hospitals }\end{array}$ \\
\hline \multicolumn{2}{|l|}{ Preoperative } \\
\hline \multicolumn{2}{|l|}{$\begin{array}{l}\text { Preoperative physical therapist intervention } \\
\text { (e.g., patient education, assessment of physical fitness and prehabilitation) }{ }^{\mathrm{b}}\end{array}$} \\
\hline Yes & $34(54.0)$ \\
\hline No & $29(46.0)$ \\
\hline \multicolumn{2}{|l|}{ Preoperative physical therapist intervention ${ }^{\mathrm{c}}$} \\
\hline Usual care & $15(44.1)$ \\
\hline Only after referral (specialist physician, case manager, nurse practitioner) & $19(55.9)$ \\
\hline \multicolumn{2}{|l|}{ Content of preoperative physical therapist intervention $(n=34)^{d}$} \\
\hline Patient education & $30(88.2)$ \\
\hline Assessment of physical fitness level & $20(58.8)$ \\
\hline Physical training (prehabilitation) & $11(32.4)$ \\
\hline
\end{tabular}

a Reported by physical therapists.

${ }^{\mathrm{b}}$ Number of hospitals in which a preoperative physical therapist intervention was part of the routine perioperative care pathway ( $n=34$; e.g., for "Yes" answer, 34/63 = 54.0\%)

"Distribution of "yes" and "no" about the preoperative referral to the physical therapy department in the 34 hospitals in which a preoperative physical therapist intervention was part of the care pathway.

${ }^{\mathrm{d}}$ Multiple answers possible. 
Table 2b. Postoperative clinical physical therapist management ${ }^{\mathrm{a}}$ for patients who underwent elective colorectal, hepatic, or pancreatic resection.

\begin{tabular}{|c|c|}
\hline Physical therapist management & $\begin{array}{l}\text { No. }(\%) \text { of } \\
\text { Hospitals }\end{array}$ \\
\hline \multicolumn{2}{|l|}{ Postoperative } \\
\hline \multicolumn{2}{|c|}{ Postoperative physical therapy as part of usual care } \\
\hline Yes & $43(68.3)$ \\
\hline No & $20(31.7)$ \\
\hline \multicolumn{2}{|c|}{ Person referring patient for postoperative physical therapy ${ }^{b}$} \\
\hline Ward physician & $46(73.0)$ \\
\hline Surgeon & $42(66.7)$ \\
\hline Nurse & $35(55.6)$ \\
\hline Physical therapist & $26(41.3)$ \\
\hline Nurse practitioner & $21(33.3)$ \\
\hline Other & $4(6.3)$ \\
\hline \multicolumn{2}{|c|}{ Postoperative physical therapist treatment frequency } \\
\hline Once a day & $47(74.6)$ \\
\hline Once or twice a day & $9(14.3)$ \\
\hline Twice a day & $5(7.9)$ \\
\hline Other (depends on individual patient) & $2(3.2)$ \\
\hline \multicolumn{2}{|c|}{ Postoperative physical therapist treatment session duration } \\
\hline 10 minutes & $9(14.3)$ \\
\hline 15 minutes & $23(36.5)$ \\
\hline 20 minutes & $30(47.6)$ \\
\hline 30 minutes & $1(1.6)$ \\
\hline \multicolumn{2}{|c|}{ Postoperative physical therapist treatment session during weekends } \\
\hline Yes & $60(95.2)$ \\
\hline No & $3(4.8)$ \\
\hline \multicolumn{2}{|l|}{ Decision for hospital discharge } \\
\hline Shared decision-making & $26(41.3)$ \\
\hline Surgeon & $22(34.9)$ \\
\hline Ward physician & $15(23.8)$ \\
\hline \multicolumn{2}{|l|}{ Specific hospital discharge criteria available } \\
\hline Yes & $42(66.7)$ \\
\hline No & $21(33.3)$ \\
\hline \multicolumn{2}{|l|}{ Specific readmission protocol available } \\
\hline Yes & $0(0.0)$ \\
\hline No & $24(38.1)$ \\
\hline Unknown & $39(61.9)$ \\
\hline \multicolumn{2}{|c|}{ Opinion concerning the usefulness of a readmission protocol } \\
\hline Useful & $3(4.8)$ \\
\hline Neutral & $29(46.0)$ \\
\hline Not useful & $31(49.2)$ \\
\hline
\end{tabular}

a Reported by physical therapists.

${ }^{b}$ Multiple answers possible. 


\section{Chapter 2}

\section{Content and variability in the reported physical therapist management}

Out of 63 respondents, $34(54.0 \%)$ reported that patients were seen preoperatively by a physical therapist, of which most patients were seen once $(31 / 34,91.2 \%)$. The content of the preoperative intervention consisted of patient education (30/34, 88.2\%), assessment of physical fitness (20/34, 58.8\%), and/or prehabilitation (11/34, 32.4\%). There was a strong uniformity between hospitals (30/34, 88.2\%) about the elements of education for patients (Figure $2 a$, graph Ia). A total of 20 respondents (20/34, 58.8\%) reported they measured the patient's preoperative physical fitness level prior to surgery. Strong uniformity concerning the importance of the assessment of daily physical activity level $(18 / 20,90.0 \%)$ and functional mobility $(17 / 20,85.0 \%)$ was observed. There was no uniformity concerning other domains of the preoperative physical fitness assessment (Figure 2a, graph Ib). No uniformity was seen for the type of preoperative physical performance tests and the type of preoperative questionnaires (Figure 2a, graph Ic). Regarding the type of exercise prehabilitation, moderate-to-low uniformity was observed for inspiratory muscle strength training $(8 / 11,72.7 \%)$, peripheral muscle strength training $(7 / 11,63.6 \%)$, and cardiorespiratory exercise training $(7 / 11,63.6 \%)$. For functional exercise training $(4 / 11,36.4 \%)$ and breathing exercises $(2 / 11,18.2 \%)$, no uniformity was observed (Figure $2 a$, graph Id). The majority of the respondents $(10 / 11$, $90.9 \%$ ) reported that not all patients were eligible for exercise prehabilitation, in which the decision for preoperative physical exercise training was based on the risk profile of the patient $(5 / 10,50.0 \%)$, the request of the surgeon $(2 / 10,20.0 \%)$, or other reasons, like the age of the patient, the number of comorbidities, or the need for inspiratory muscle training $(3 / 10,30.0 \%)$.

Postoperative physical therapist practice of which (very) strong uniformity was observed between the respondents $(n=63)$ were breathing exercises $(62 / 63,98.4 \%)$, practicing transfers $(62 / 63,98.4 \%)$, patient education $(61 / 63,96.8 \%)$, stair climbing (56/63, $88.8 \%$ ), and walking exercises (55/63, 87.3\%) (Figure 2b, graph IIa). Low or no uniformity was observed for postoperative physical performance tests to monitor and evaluate the patient's recovery of physical functioning (Figure $2 b$, graph IIb). 
Ia. Elements of education for patients $(n=30,30 / 34,88.2 \%)$

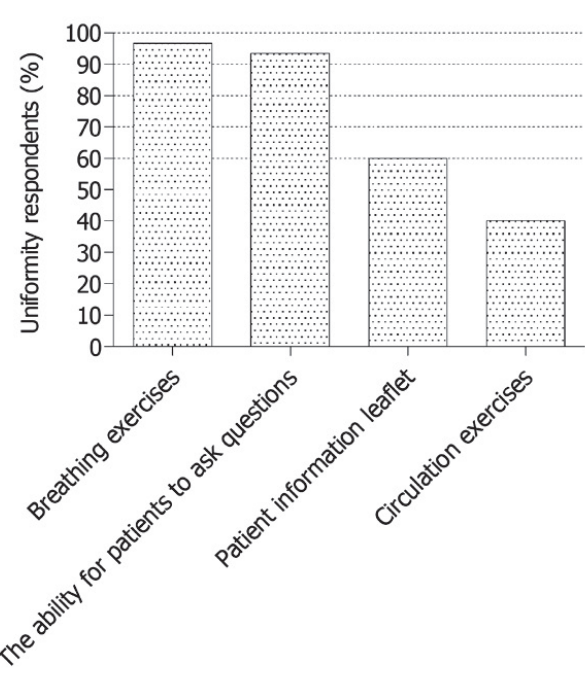

Ic. Type of performance tests used $(n=16,16 / 34,47.1 \%)$
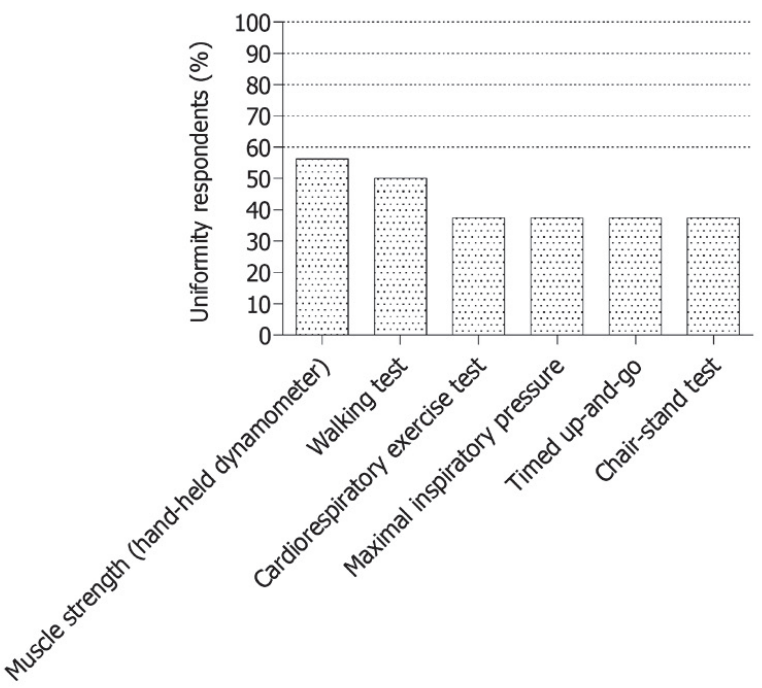

Ib. Domains of physical fitness assessed $(n=20,20 / 34,58.8 \%)$
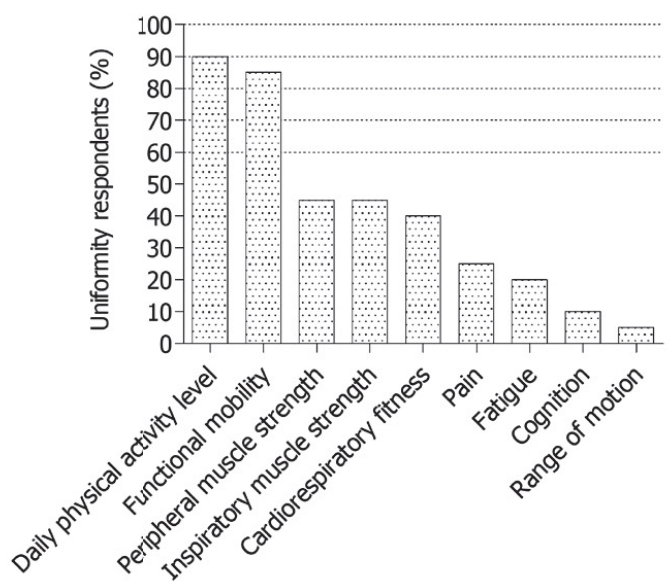

Id. Type of exercise prehabiliation $(n=11,11 / 34,32.4 \%)$

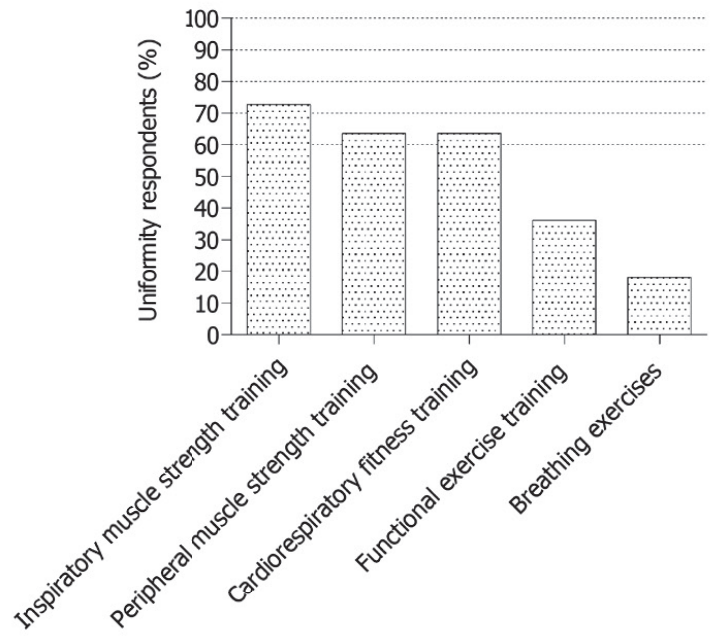

Figure 2a. Reported physical therapist interventions performed by physical therapists in patients opting for elective colorectal, hepatic, or pancreatic resection, including preoperative physical therapist interventions $(n=34)$, elements of education (Ia, $n=30)$, domains of physical fitness assessment used by physical therapists (Ib, $n=20$ ), type of performance tests used to assess physical fitness (Ic, $n=16)$, and components of exercise prehabilitation (Id, $n=11$ ). 
II. Reported postoperative physical therapist management ( $n=63,63 / 63,100 \%)$

IIa. Content of postoperative physical therapy $(n=63,63 / 63,100.0 \%)$
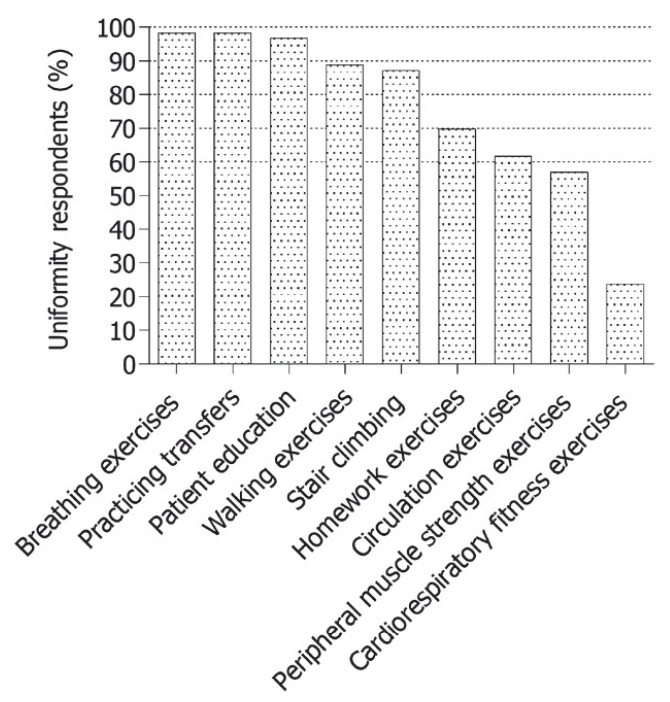

IIb. Postoperative performance tests $(n=25,25 / 63,39.7 \%)$

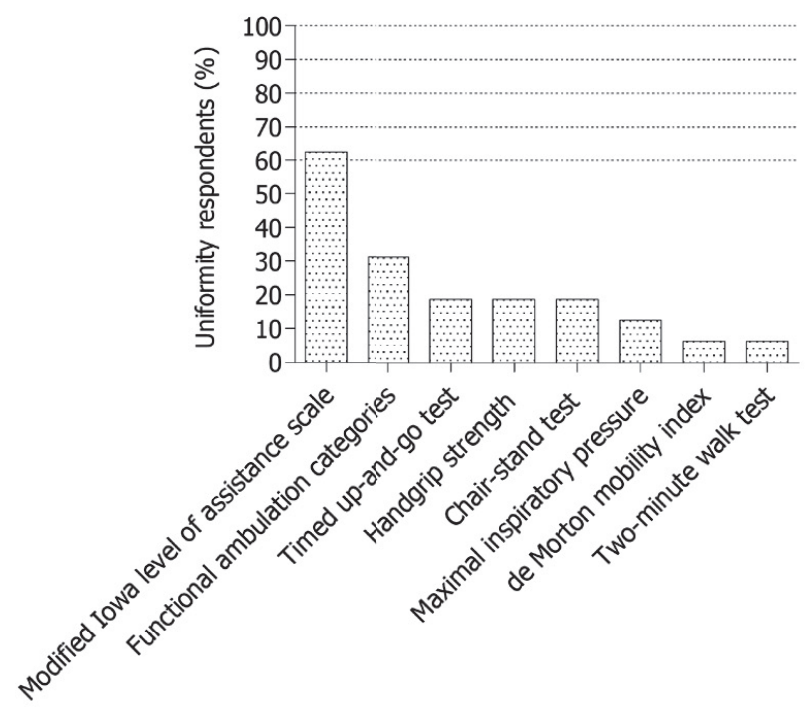

Figure 2b. Reported physical therapist interventions performed by physical therapists in patients following elective colorectal, hepatic, or pancreatic resection, including postoperative physical therapist interventions ( $n=63)$; content of postoperative physical therapist treatment (IIa, $n=63$ ) and postoperative performance tests for monitoring the patient's outcome (IIb, $n=25)$. 


\section{Comparison between reported physical therapist management and advised state-of-the-art physical therapy}

LCA identified a three-class model based on the AIC (988.52), BIC (1102.10) and substantive relevance. The fit-indices from the LCA are reported in Table 3. LCA assigned each hospital to one of the three classes (posterior probability) and calculated the conditional probabilities (value between 0 and 1 ) of each item for each class (Table 4). For the items addressing preoperative physical therapy, respondents in class I and II were more likely to report that they provide preoperative physical therapy for patients than respondents in class III. There was a $94.0 \%$ chance that a hospital in latent class I provided a preoperative assessment of physical fitness and a $50.0 \%$ chance that this respondent provided prehabilitation, whereas these probabilities for respondents in class II were $28.0 \%$ (providing a preoperative assessment) and $17.0 \%$ (providing prehabilitation), respectively. For postoperative physical therapist management, no major differences were seen in the conditional probabilities between the three classes. Calculating the index scores for each hospital, meaning the distance between the reported physical therapist practice and advised state-of-the-art physical therapy, the same patterns as the LCA classes are observed in the pre- and postoperative physical therapy. Figure 3 shows the index scores for each hospital. Additionally, the average response profiles for each group on the 19-item dichotomous scale are graphically presented in Figure 3 ( $a, b, c$, and d).

Table 3. The fit-indices of the latent class analysis models for the 19-item dichotomous scale.

\begin{tabular}{|c|c|c|c|c|c|}
\hline Nclass & Log-likelihood & BIC & AIC & Df & Npar \\
\hline 1 & -565.7281 & 1201.889 & 1165.4562 & 46 & 17 \\
\hline 2 & -474.4141 & 1093.838 & 1018.8282 & 28 & 35 \\
\hline 3 & -441.2581 & 1102.102 & 988.5162 & 10 & 53 \\
\hline 4 & -430.8282 & 1155.819 & 1003.6563 & -8 & 71 \\
\hline
\end{tabular}

Abbreviations: AIC=Akaike's information criterion; BIC=Schwarz's Bayesian information criterion; $\mathrm{Df}=$ degrees of freedom; Nclass=number of classes; Npar=number of parameters. 
Table 4. Descriptive statistics for each item on the 19-item dichotomous scale and class response probability ${ }^{\mathrm{a}}$.

\begin{tabular}{|c|c|c|c|c|c|}
\hline & & & $\begin{array}{c}\text { Class } \\
\text { I }\end{array}$ & $\begin{array}{c}\text { Class } \\
\text { II }\end{array}$ & $\begin{array}{c}\text { Class } \\
\text { III }\end{array}$ \\
\hline \multicolumn{3}{|l|}{ Predicted class membership (posterior probability) } & 0.25 & 0.29 & 0.46 \\
\hline Preoperative physical therapist management & $n$ & $(\%)$ & \multicolumn{3}{|c|}{ Class response probabilities } \\
\hline $\begin{array}{l}\text { 1. Patients visit the physical therapist } \\
\text { preoperatively }\end{array}$ & 34 & $(54.0)$ & 1.00 & 1.00 & 0.00 \\
\hline $\begin{array}{l}\text { 2. The physical therapist provides patient } \\
\text { education }\end{array}$ & 30 & $(47.6)$ & 0.88 & 0.89 & 0.00 \\
\hline $\begin{array}{l}\text { 3. The physical therapist assesses the patient's } \\
\text { physical fitness level }\end{array}$ & 20 & $(31.7)$ & 0.94 & 0.28 & 0.00 \\
\hline $\begin{array}{l}\text { 4. Questionnaires are used in the preoperative } \\
\text { assessment }\end{array}$ & 16 & $(25.4)$ & 1.00 & 0.00 & 0.00 \\
\hline $\begin{array}{l}\text { 5. Physical performance tests are used in the } \\
\text { preoperative assessment }\end{array}$ & 10 & $(15.9)$ & 0.63 & 0.00 & 0.00 \\
\hline $\begin{array}{l}\text { 6. The physical therapist provides exercise } \\
\text { prehabilitation }\end{array}$ & 11 & $(17.5)$ & 0.50 & 0.17 & 0.00 \\
\hline $\begin{array}{l}\text { 7. The context of the prehabilitation is at the } \\
\text { patient's home }\end{array}$ & 8 & $(12.7)$ & 0.44 & 0.00 & 0.00 \\
\hline Postoperative physical therapist management & $n$ & $(\%)$ & \multicolumn{3}{|c|}{ Class response probabilities } \\
\hline $\begin{array}{l}\text { 8. A multimodal perioperative care pathway } \\
\text { (e.g., ERAS }{ }^{\mathrm{b}} \text {, fast-track) is implemented and a } \\
\text { postoperative protocol is followed }\end{array}$ & 43 & $(68.3)$ & 0.75 & 0.72 & 0.62 \\
\hline $\begin{array}{l}\text { 9. The physical therapist treatment is initiated by } \\
\text { the physical therapist }\end{array}$ & 26 & $(41.3)$ & 0.50 & 0.44 & 0.34 \\
\hline $\begin{array}{l}\text { 10. The physical therapist treatment starts at the } \\
\text { day of surgery }\end{array}$ & 10 & $(15.9)$ & 0.13 & 0.28 & 0.10 \\
\hline $\begin{array}{l}\text { 11. Physical therapy is structurally continued } \\
\text { during the weekends }\end{array}$ & 11 & $(17.5)$ & 0.13 & 0.33 & 0.10 \\
\hline $\begin{array}{l}\text { 12. The physical therapist treatment is mainly } \\
\text { performed in the living room of the surgical } \\
\text { nurse ward c }\end{array}$ & 0 & $(0.0)$ & - & - & - \\
\hline $\begin{array}{l}\text { 13. If necessary, treatment frequency can be } \\
\text { adapted to the patient's need }\end{array}$ & 62 & $(98.4)$ & 1.00 & 1.00 & 0.97 \\
\hline $\begin{array}{l}\text { 14. To monitor postoperative outcomes, } \\
\text { questionnaires are used }\end{array}$ & 9 & $(14.3)$ & 0.13 & 0.06 & 0.21 \\
\hline $\begin{array}{l}\text { 15. To monitor postoperative outcomes, physical } \\
\text { performance tests are used }\end{array}$ & 16 & $(25.4)$ & 0.56 & 0.06 & 0.21 \\
\hline Discharge and readmission & $n$ & $(\%)$ & & & \\
\hline $\begin{array}{l}\text { 16. Discharge performed by shared } \\
\text { decisionmaking }\end{array}$ & 26 & $(41.3)$ & 0.56 & 0.56 & 0.24 \\
\hline 17. Discharge criteria are available & 42 & $(66.7)$ & 0.88 & 0.72 & 0.52 \\
\hline 18. A readmission protocol is available ${ }^{c}$ & 0 & $(0.0)$ & - & - & - \\
\hline $\begin{array}{l}\text { 19. In case of a readmission, the physical } \\
\text { therapist is timely consulted }\end{array}$ & 48 & $(76.2)$ & 0.75 & 0.67 & 0.82 \\
\hline
\end{tabular}

a Probability of reporting "yes" on each item of each class.

${ }^{\mathrm{b}}$ ERAS=enhanced recovery after surgery.

cThis item contained only 1 outcome category ("no"-response), and was removed from the analysis. 
Index score calculated on the 7-items for preoperative physical therapist management for each hospital ( $n=63)$

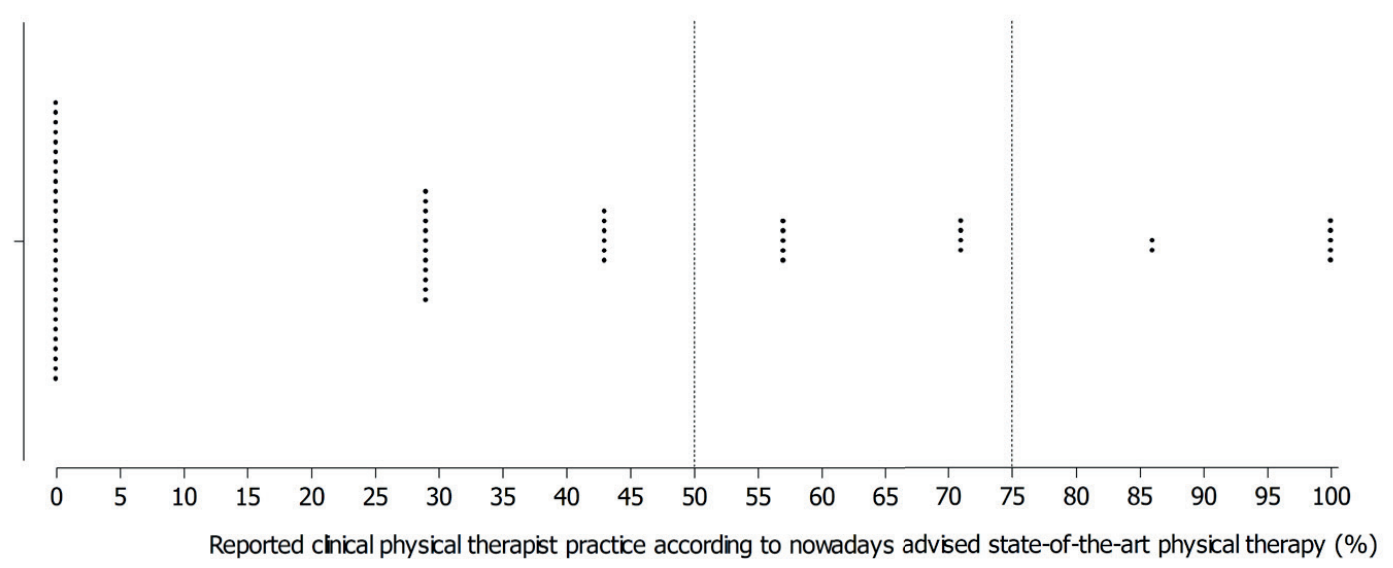

Average response profile for sub-groups - Preoperative physical therapy

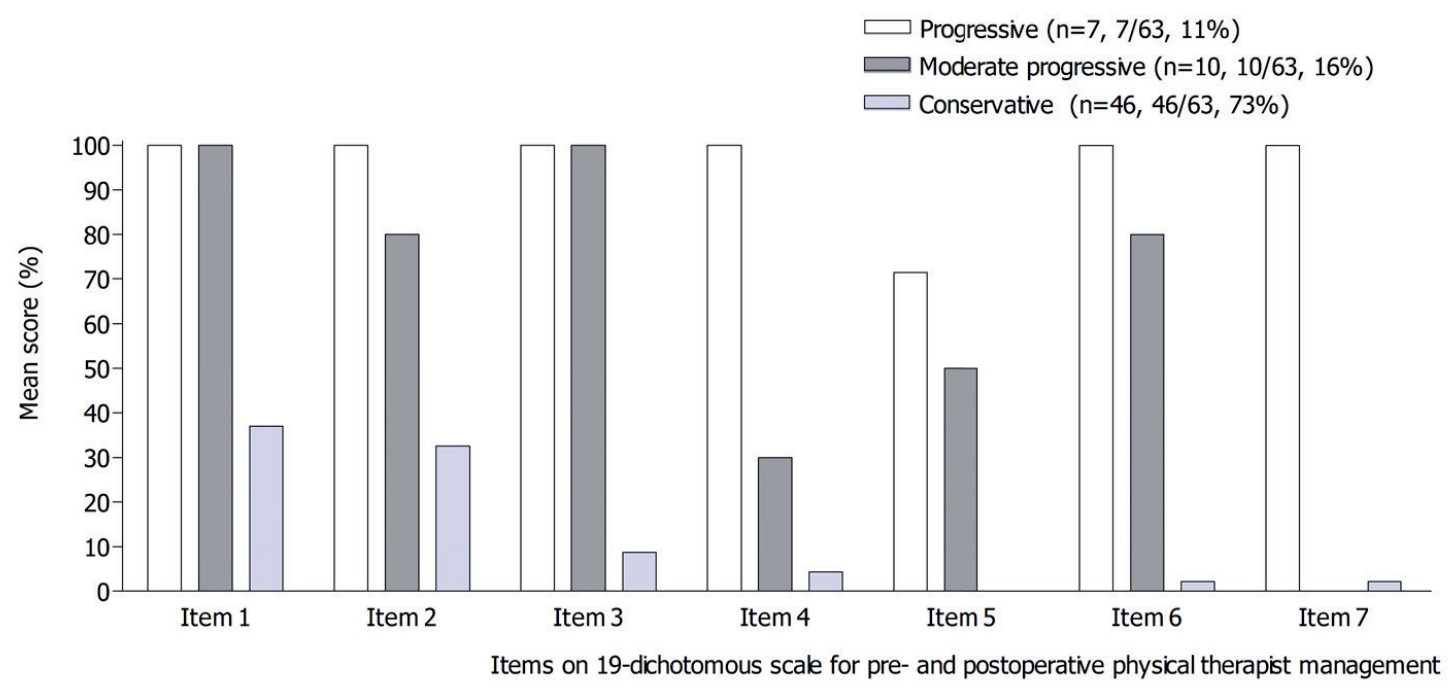

Figure 3a. The index scores (frequency distribution) for each hospital and average response profiles for each group on the 19-item dichotomous scale. 


\section{Postoperative physical therapist management}

\section{Index score calculated on the 8-items for postoperative physical therapist management for each hospital ( $n=63$ )}

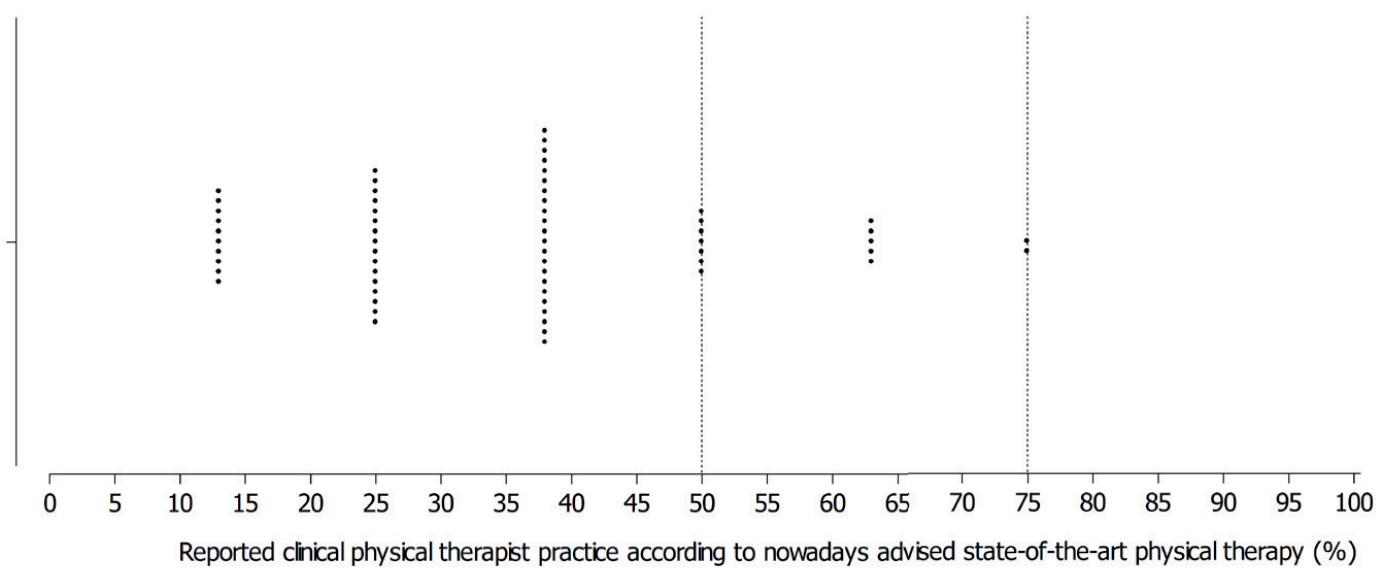

Average response profile for sub-groups - Postoperative physical therapy

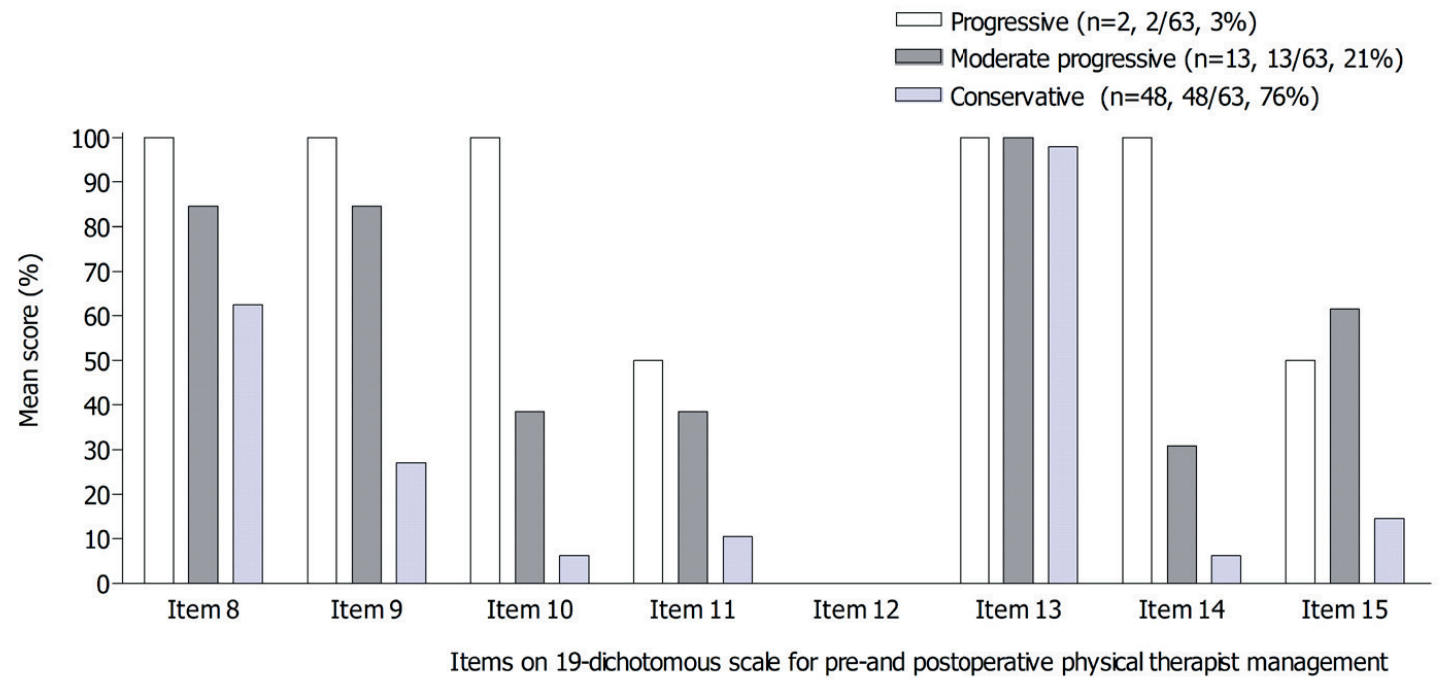

Figure 3b. The index scores (frequency distribution) for each hospital and average response profiles for each group on the 19-item dichotomous scale. 


\section{Discharge and readmission}

Index score calculated on the 4-items for discharge and readmission for each responding hospital $(n=63)$
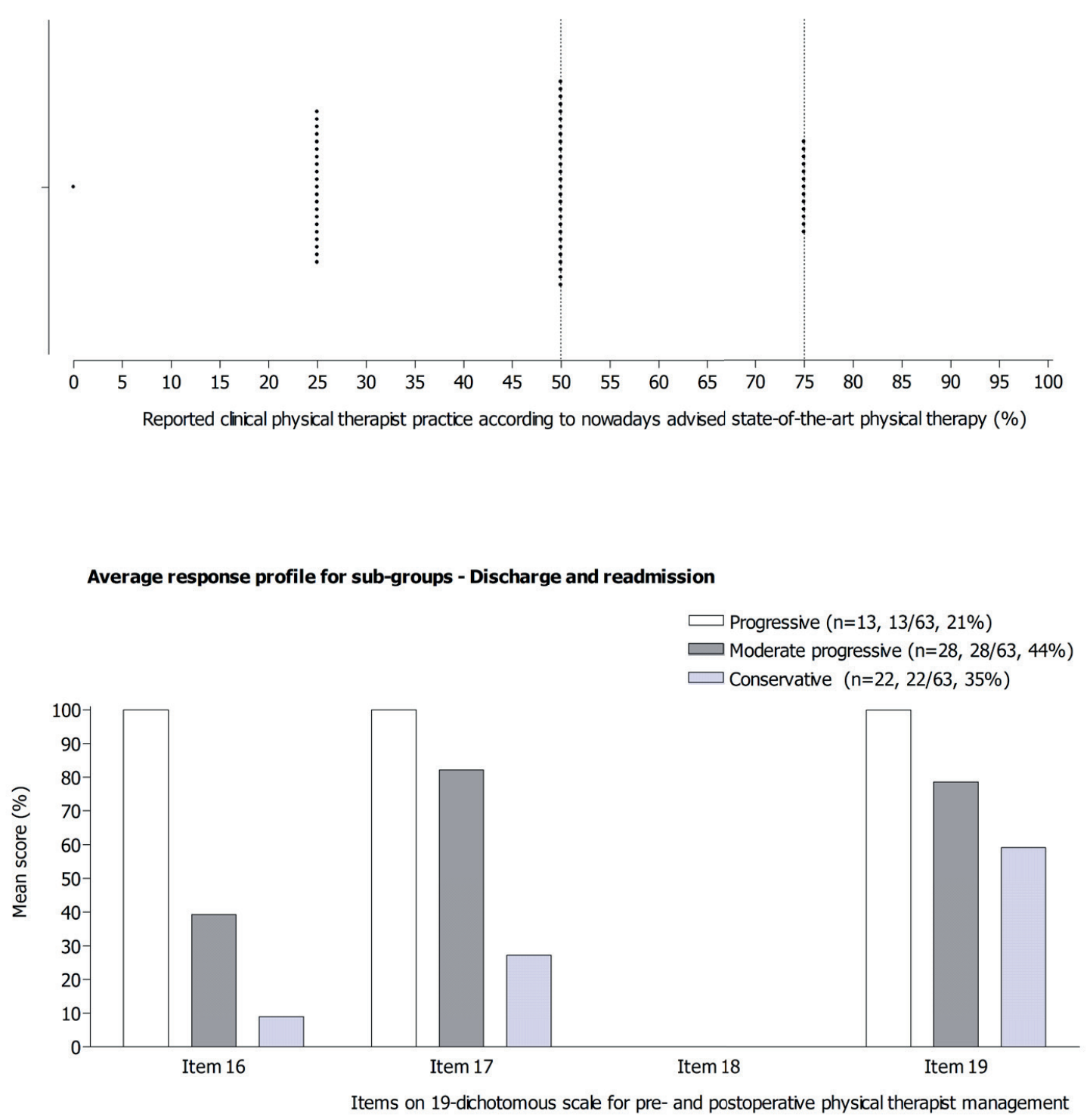

Figure 3c. The index scores (frequency distribution) for each hospital and average response profiles for each group on the 19-item dichotomous scale. 
Pre- and postoperative physical therapist management

Index score calculated on the 19-items for pre-and postoperative physical therapy for each hospital ( $n=63$ )

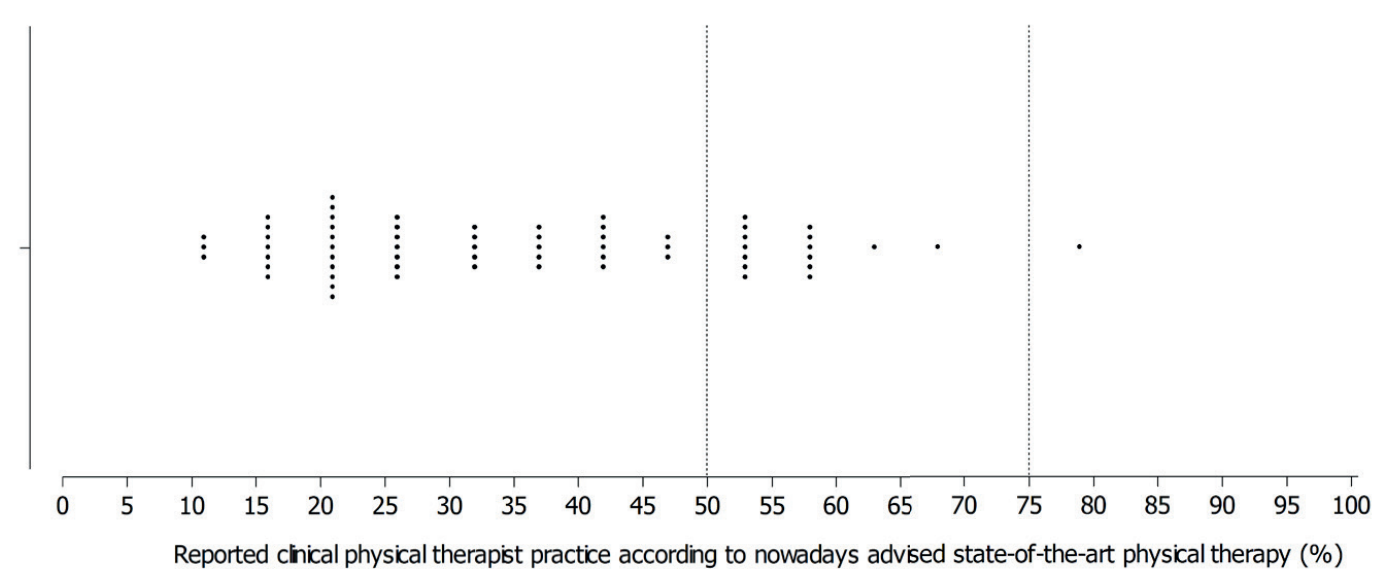

Average response profile for sub-groups pre-and postoperative physical therapist management

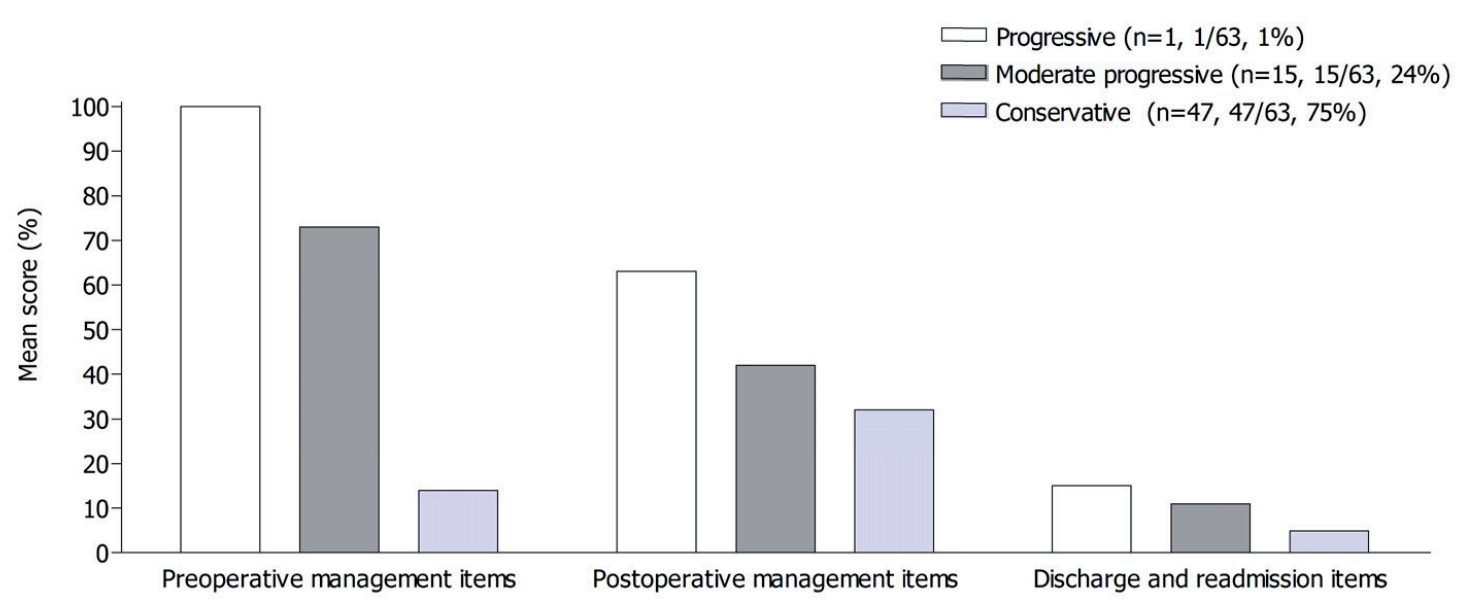

Figure 3d. The index scores (frequency distribution) for each hospital and average response profiles for each group on the 19-item dichotomous scale. 


\section{DISCUSSION}

This study provided an overview of the current clinical physical therapist management (response rate $65 / 82,79.3 \%$ ) in patients who opt for abdominal surgery for colorectal, hepatic, or pancreatic cancer in the Netherlands. The reported content and between hospital variability was described, and moderate-to-strong uniformity among physical therapists was reported regarding preoperative education and postoperative physical therapist treatment goals. For pre- and postoperative performance measures, no uniformity was found. In addition, three different classes of clinical physical therapist practice were identified by comparing the nowadays-advised state-of-the-art physical therapy and the self-reported physical therapist management.

The first aim of this study was to describe the current content and variability in pre- and postoperative physical therapist management in major abdominal surgery. A response rate $>75 \%$ is considered excellent, which makes the participant responses to our survey fairly representative for the current Dutch clinical practice. Besides, comparable studies reported similar response rates of $80.7 \%$ (46/57) [29] and $82.4 \%$ (28/34) [26]. For this study, physical therapists working on a daily base with patients opting for major elective abdominal surgery were consulted, who therefore have extensive knowledge concerning the current practice in the respective hospital. Consequently, this study provided rather accurate information about the current pre- and postoperative physical therapist management in the respective hospitals.

Variability between the respondents was assessed by evaluating the level of uniformity in physical therapist management. In 53.9\% (34/63) of the respondents, patients were seen preoperatively by a physical therapist, in which uniformity existed in the provided elements of education for patients, but not concerning the different domains of physical fitness assessed. Additional descriptive analysis did not demonstrate clear differences between academic and general hospitals. Screening of patients prior to major abdominal surgery by physical therapists is not yet routine physical therapist practice in the Netherlands $[9,10]$. Similar results were reported in surveys in other surgical populations $[23,25,30-32]$. Previously reported potential barriers for preoperative physical therapy were lack of time, insufficient evidence, or the fact that relevant information was already provided by other health care professionals [32]. Concerning postoperative physical therapy, this study indicated strong uniformity between respondents concerning airway clearing exercises, patient education (stimulating self-management), early mobilization, and practicing functional tasks (e.g., transfers, walking exercises, and stair climbing). Airway clearing exercises were performed in the majority of the hospitals (62/63, 98.4\%); however, the literature remains inconclusive about the type of breathing exercises and the additional effects of breathing exercises compared to mobilization alone in major (upper) abdominal surgery [33,34]. Of the respondents, $38.1 \%$ reported that there is no readmission protocol available at their hospital. However, $61.9 \%$ of the 
respondents is unaware about the availability of such a protocol in their hospital. Nevertheless, $76.2 \%(48 / 63)$ of the respondents reported to be consulted in time when a readmission occurred. It is important to react adequately when a readmission occurs, as a readmission is associated with additional short-term morbidity and mortality in major abdominal surgery [35].

The second aim of the study was to compare the nowadays-advised state-of-the-art physical therapy with the self-reported daily clinical physical therapist practice. Using LCA on the reported data about pre- and postoperative physical therapy, three classes of practice were identified, in which hospitals in class I and class II were more likely to provide preoperative risk assessment; however, prehabilitation was most likely to be offered in class I. Therefore, class I is most closely related to the nowadays-advised state-of-the-art physical therapy and are classified as "progressive". Respondents who reported to merely provide preoperative risk assessment are more likely to belong to class II ("moderately progressive"). Respondents that do not provide preoperative physical therapy (risk assessment and prehabilitation) have the biggest chance to belong to class III ("conservative"). These respondents can learn from experiences of class I and II when aiming to implement state-of-the-art evidence- and practice-based preoperative interventions.

The importance of a preoperative assessment of physical fitness in the prediction of postoperative outcomes is well documented nowadays [36]. Cardiopulmonary exercise testing is the gold standard for assessing cardiorespiratory fitness and has been found to have a consistent relation with postoperative outcomes (e.g., morbidity, length of stay, mortality) in major abdominal surgery $[37,38]$. Clear cut-off values for cardiorespiratory fitness to preoperatively identify patients with a higher risk for postoperative morbidity have been published [39]. However, performing cardiopulmonary exercise testing is not always feasible as usual care in the Netherlands. Hereto, more practical performancebased tests to estimate cardiorespiratory fitness can be used (e.g., incremental shuttle walk test, timed up-and-go test) [40,41]; however, they still need further validation [39]. The latter might explain the lack of uniformity between the respondents using these type of tests in their preoperative physical fitness assessment. Moreover, besides measuring cardiorespiratory fitness, it is important to preoperatively assess muscle strength and functional mobility, as these components of physical fitness are a prerequisite for (early) mobilization and being physically active during the postoperative phase [42].

It is encouraging to see that $17.5 \%$ of the respondents $(11 / 63)$ provide exercise prehabilitation prior to major abdominal surgery. Recent studies in major abdominal surgery demonstrated that prehabilitation (in high-risk patients) improves the preoperative physical fitness level and can reduce the risk for postoperative complications, as well as reduce length of stay [43-45]. Despite these reported beneficial effects, most studies evaluating the effectiveness of prehabilitation hamper preoperative 
risk assessment that might lead to selection bias (inclusion of mainly low-risk patients) [46]. Moreover, studies are too heterogeneous concerning the context (home-, community-, or hospital-based) and content (frequency, intensity, time, and type) of the preoperative exercise training program, making further research necessary [46].

Concerning postoperative physical therapist management, no major differences were seen in the conditional probabilities between the three classes. No probability could be calculated from item 12 and 18 , as there was no variability within the item responses. Postoperatively, early mobilization and practicing functional tasks are part of the ERAS care pathway, as implemented in $63.0 \%$ of the respondents. Implementation of ERAS programs resulted in major improvements in clinical outcomes and costs in colorectal, hepatic, and pancreatic surgery [47-49]. Although the importance of early mobilization and the physiological and functional consequences of bed-rest are known [50], it has been reported that patients spent about $83.0 \%$ of their time in bed, often without a medical reason [51]. The use of the ERAS care pathway with a multidisciplinary patientcentered approach in combination with a physically activating culture and infrastructure has been advocated to improve and accelerate the recovery of physical functioning postoperatively $[9,10]$. The low probabilities for the use of performance-based tests to monitor the patient's recovery of physical functioning and the availability of clear (clinimetric) discharge criteria in hospitals is remarkable. Literature describes that frequent monitoring of the recovery of physical functioning, ideally using performancebased tests, during the treatment course is very important for guiding the care process and discharge planning [52].

Implementation of state-of-the-art pre- and postoperative physical therapist management is a complex intervention. A pragmatic strategy in which implementation consists of an interaction between the implementation process, context, and outcomes might be valuable [53]. First, the physical therapist should be aware of the available evidence and the related recommendations for clinical practice. Then, in an iterative process, small changes can be made in daily clinical practice with real-time monitoring of relevant outcomes based on qualitative and quantitative data. This real-time feedback driven adaptations are necessary to make changes in daily clinical practice sustainable [53,54]. An example for such an approach is published by van der Sluis et al. [22].

\section{Limitations of the study}

A limitation of this study is uncertainness concerning the generalizability of the used literature to describe state-of-the-art physical therapist management. Patient characteristics of the participating hospitals were unknown, making it not possible to evaluate how well the patient population described in the literature fits the patient population of the participating hospitals. The survey was not evaluated on reliability and 


\section{Chapter 2}

validity and there was no structured assessment on the interpretation of the questions. Finally, the use of self-reported answers might lead to bias towards real-life practice.

There is a wide degree of variability between hospitals regarding pre-and postoperative clinical physical therapist practice for patients opting for major elective abdominal surgery. Three classes of clinical physical therapist practice were identified, differing in adherence to the evidence provided in the literature. It is encouraging to see current developments (e.g., preoperative risk assessment and prehabilitation) in daily clinical practice related to evidence- and practice-based conceptual models as described in the literature to further improve perioperative (physical therapy) care for these patients. Further translation of key research findings into clinical physical therapist practice is advised, especially for hospitals in which the physical therapist is not involved preoperatively. Moreover, improving uniformity by developing up-to-date clinical guidelines is recommended. 


\section{REFERENCES}

1. World Health Organization, Global health and aging report. http://www.who.int/ageing/publications /global_health/en/, 2011 (accessed October 2016).

2. Makary MA, Segev DL, Pronovost PJ, et al., Frailty as a predictor of surgical outcomes in older patients, J Am Coll Surg. 210 (2010) 901-908.

3. Yang R, Wolfson M, Lewis MC, Unique aspects of the elderly surgical population: an anesthesiologist's perspective, Geriatr Orthop Surg Rehab. 2 (2011) 56-64.

4. Lin HS, Watts JN, Peel NM, et al., Frailty and post-operative outcomes in older surgical patients: a systematic review, BMC Geriatr. 16 (2016) 157.

5. Nicholson A, Lowe MC, Parker J, et al., Systematic review and meta-analysis of enhanced recovery programmes in surgical patients, Br J Surg. 101 (2014) 172-188.

6. Finnerty CC, Mabvuure NT, Ali A, et al., The surgically induced stress response, JPEN J Parenter Enteral Nutr. 37 (2013) 21S-29S.

7. McEwen BS, Wingfield JC, What is in a name? Integrating homeostasis, allostasis and stress, Horm Behav. 57 (2010) 105-111.

8. Kim S, Brooks AK, Groban L, Preoperative assessment of the older surgical patient: honing in on geriatric syndromes, Clin Interv Aging. 10 (2014) 13-27.

9. Hulzebos EH, van Meeteren NL, Making the elderly fit for surgery, Br J Surg. 103 (2016) e12-15.

10. Hulzebos EH, van Meeteren NL, Making the elderly fit for surgery, Br J Surg. 103 (2016) 463.

11. Wynter-Blyth V, Moorthy K, Prehabilitation: preparing patients for surgery, BMJ. 358 (2017) j3702.

12. Lopopolo RB, Hospital restructuring and the changing nature of the physical therapist's role, Phys Ther. 79 (1999) 171-185.

13. Hood L, Systems biology and p4 medicine: past, present, and future, Rambam Maimonides Med J. 4 (2013) e0012.

14. Bongers BC, Dronkers JJ, Hulzebos $\mathrm{HJ}$, et al., Optimizing perioperative physical therapy care in major elective surgery to improve surgical outcome in high-risk patients: the Better in, Better out ${ }^{\mathrm{TM}}$ concept, $^{-}$ NTvA. 29 (2016) 134-139.

15. Tew GA, Ayyash R, Durrand J, et al., Clinical guideline and recommendations on pre-operative exercise training in patients awaiting major non-cardiac surgery, Anaesthesia. 73 (2018) 750-768.

16. Lassen K, Soop M, Nygren J, et al., Consensus review of optimal perioperative care in colorectal surgery: enhanced recovery after surgery (ERAS) group recommendations, Arch Surg. 144 (2009) 961-969.

17. Lassen K, Coolsen MM, Slim K, et al., Guidelines for perioperative care for pancreaticoduodenectomy: enhanced recovery after surgery (ERAS $\left.{ }^{\circ}\right)$ society recommendations, Clin Nutr. 31 (2012) 817-830.

18. Hulzebos EH, Smit Y, Helders PP, et al., Preoperative physical therapy for elective cardiac surgery patients, Cochrane Database Syst Rev. 11 (2012) CD010118.

19. Hulzebos EH, Helders PJ, Favie NJ, et al., Preoperative intensive inspiratory muscle training to prevent postoperative pulmonary complications in high-risk patients undergoing CABG surgery: a randomized clinical trial, JAMA. 296 (2006) 1851-1857.

20. Dronkers JJ, Lamberts $\mathrm{H}$, Reutelingsperger IM, et al., Preoperative therapeutic programme for elderly patients scheduled for elective abdominal oncological surgery: a randomized controlled pilot study, Clin Rehabil. 24 (2010) 614-622.

21. Minnella EM, Bousquet-Dion G, Awasthi $R$, et al., Multimodal prehabilitation improves functional capacity before and after colorectal surgery for cancer: a five-year research experience, Acta Oncol. 56 (2017) 295-300.

22. van der Sluis G, Goldbohm RA, Bimmel R, et al., What augmented physical activity and empowerment can bring to patients receiving total knee replacement: content, implementation, and comparative 


\section{Chapter 2}

effectiveness of a new function-tailored care pathway in a routine care setting, BioMed Res Int. 2015 (2015) 745864.

23. Reeve J, Denehy L, Stiller K, The physiotherapy management of patients undergoing thoracic surgery: a survey of current practice in Australia and New Zealand, Physiother Res Int. 12 (2007) 59-71.

24. Naylor J, Harmer A, Fransen $M$, et al., Status of physiotherapy rehabilitation after total knee replacement in Australia, Physiother Res Int. 11 (2006) 35-47.

25. Westerdahl E, Moller M, Physiotherapy-supervised mobilization and exercise following cardiac surgery: a national questionnaire survey in Sweden, J Cardiothorac Surg. 5 (2010) 67.

26. Janssen ERC, Scheijen EE, van Meeteren NL, et al., Determining clinical practice of expert physiotherapy for patients undergoing lumbar spinal fusion: a cross-sectional survey study, Eur Spine J. 25 (2016) 1533-1541.

27. Peter WF, Nelissen RG, Vlieland TP, Guideline recommendations for post-acute postoperative physiotherapy in total hip and knee arthroplasty: are they used in daily clinical practice? Musculoskeletal care. 12 (2014) 125-131.

28. Linzer DA, Lewis JB, poLCA: An R package for polytomous variable latent class analysis, J Stat Softw. 42 (2011) 1-29.

29. Bougeard AM, Brent A, Swart M, et al., A survey of UK peri-operative medicine: pre-operative care, Anaesthesia. 72 (2017) 1010-1015.

30. Patman S, Bartley A, Ferraz A, et al., Physiotherapy in upper abdominal surgery - what is current practice in Australia? Arch Physiother. 7 (2017) 11.

31. Cavalheri V, Jenkins S, Hill K, Physiotherapy practice patterns for patients undergoing surgery for lung cancer: a survey of hospitals in Australia and New Zealand, Intern Med J. 43 (2013) 394-401.

32. Reeve J, Denehy L, Stiller K, The physiotherapy management of patients undergoing thoracic surgery: a survey of current practice in Australia and New Zealand, Physiother Res Int. 12 (2007) 59-71.

33. Reeve J, Boden I, The physiotherapy management of patients undergoing abdominal surgery, New Zealand J Physiother. 44 (2016) 33-49.

34. Silva YR, Li SK, Rickard MJ, Does the addition of deep breathing exercises to physiotherapy-directed early mobilisation alter patient outcomes following high-risk open upper abdominal surgery? Cluster randomised controlled trial, Physiotherapy. 99 (2013) 187-193.

35. Schneider EB, Hyder $\mathrm{O}$, Wolfgang $\mathrm{CL}$, et al., Patient readmission and mortality after surgery for hepato-pancreato-biliary malignancies, J Am Coll Surg. 215 (2012) 607-615.

36. Levett DZ, Edwards M, Grocott M, et al., Preparing the patient for surgery to improve outcomes, Best Pract Res Clin Anaesthesiol. 30 (2016) 145-157.

37. West MA, Asher R, Browning M, et al., Validation of preoperative cardiopulmonary exercise testingderived variables to predict in-hospital morbidity after major colorectal surgery, Br J Surg. 103 (2016) 744-752.

38. Levett DZ, Jack S, Swart M, et al., Perioperative cardiopulmonary exercise testing (CPET): consensus clinical guidelines on indications, organization, conduct, and physiological interpretation, $\mathrm{Br} \mathrm{J}$ Anaesth. 120 (2018) 484-500.

39. Moran J, Guinan E, McCormick P, et al., The ability of prehabilitation to influence postoperative outcome after intra-abdominal operation: a systematic review and meta-analysis, Surgery. 160 (2016) 1189-1201.

40. Nutt CL, Russell JC, Use of the pre-operative shuttle walk test to predict morbidity and mortality after elective major colorectal surgery, Anaesthesia. 67 (2012) 839-849.

41. Heldens $A F$, Bongers $B C$, Lenssen $A F$, et al., The association between performance parameters of physical fitness and postoperative outcomes in patients undergoing colorectal surgery: an evaluation of care data, Eur J Surg Oncol. 43 (2017) 2084-2092. 
42. Punt IM, Bongers BC, van Beijsterveld CA, et al., Surgery: Moving people, improving outcomes, in: Geriatrics, Avid Science, India, 2016, pp. 2-30.

43. Barberan-Garcia A, Ubré M, Roca J, et al., Personalised prehabilitation in high-risk patients undergoing elective major abdominal surgery: a randomized blinded controlled trial, Ann Surg. 267 (2018) 50-56.

44. Bolshinsky V, Li MH, Ismail $\mathrm{H}$, et al., Multimodal prehabilitation programs as a bundle of care in gastrointestinal cancer surgery: a systematic review, Dis Colon Rectum. 61 (2018) 124-138.

45. West MA, Wischmeyer PE, Grocott MP, Prehabilitation and nutritional support to improve perioperative outcomes, Curr Anesthesiol Rep. 7 (2017) 340-349.

46. Berkel $A E$, Bongers $B C$, van Kamp MS, et al., The effects of prehabilitation versus usual care to reduce postoperative complications in high-risk patients with colorectal cancer or dysplasia scheduled for elective colorectal resection: study protocol of a randomized controlled trial, BMC Gastroenterol. 18 (2018) 29.

47. Ljungqvist O, Scott M, Fearon KC, Enhanced recovery after surgery: a review, JAMA Surg. 152 (2017) 292-298.

48. Melloul E, Hübner M, Scott M, et al., Guidelines for perioperative care for liver surgery: enhanced recovery after surgery (ERAS) society recommendations, World J Surg. 40 (2016) 2425-2440.

49. Zhuang $C L$, Ye $X Z$, Zhang $X D$, et al., Enhanced recovery after surgery programs versus traditional care for colorectal surgery: a meta-analysis of randomized controlled trials, Dis Colon Rectum. 56 (2013) 667-678.

50. Sourdet S, Lafont $C$, Rolland $Y$, et al., Preventable iatrogenic disability in elderly patients during hospitalization, J Am Med Dir Assoc. 16 (2015) 674-681.

51. Brown $\mathrm{CJ}$, Redden $\mathrm{DT}$, Flood $\mathrm{KL}$, et al., The underrecognized epidemic of low mobility during hospitalization of older adults, J Am Geriatr Soc. 57 (2009) 1660-1665.

52. Glasziou P, Irwig L, Mant D, Monitoring in chronic disease: a rational approach, BMJ. 330 (2005) 644-648.

53. Luig T, Asselin J, Sharma AM, et al., Understanding implementation of complex interventions in primary care teams, J Am Board Fam Med. 31 (2018) 431-444.

54. Moore GF, Audrey S, Barker M, et al., Process evaluation of complex interventions: medical research council guidance, BMJ. 350 (2015) h1258. 


\section{Chapter 2}

\section{SUPPLEMENTARY INFORMATION}

Supplemental file 1. E-survey: evaluating current clinical physical therapist practice for patients undergoing elective colorectal, hepatic, or pancreatic resection. Additional clarification of the used terminology can be found on the last page.

This e-survey is meant to construct an overview of the current clinical practice of physical therapists in adult patients undergoing elective colorectal, hepatic, or pancreatic resection in the Netherlands. Results will be analysed anonymously and will be used for publication. We kindly request you to complete this e-survey.

\section{Background information}

1. In what type of hospital do you work?
- Academic hospital
- General hospital
- Specialized health care centre
o Other,

2. What is the name of the hospital you work in?

3. Is there a protocol available at your institution for treating patients undergoing major elective abdominal surgery?
o Yes
$\circ$ No

4. Are you willing to make your hospital's protocol for treating patients undergoing major elective abdominal surgery available to us? The document will be analysed anonymously. If yes, please provide us with your e-mail address, so we can contact you.
$\circ$ Yes, my e-mail address is
○ No
- Not applicable

5. How many physical therapist work at the physical therapy department of your hospital? physical therapists with a Bachelor of Applied Science degree physical therapists with a Bachelor of Applied Science and specialization (e.g., neurology, oncology, pediatrics)
_ physical therapists with a Master of Science degree
_ physical therapists with a Doctor of Philosophy degree
_ physical therapist students 


\section{Preoperative physical therapist management}

6. Are patients undergoing major elective abdominal surgery preoperatively seen by a physical therapist at your hospital?
$\circ$ Yes
$\circ$ No

7. Are all patients preoperatively seen by a physical therapist?
$\circ$ Yes
- No, only when referred by a specialist physician, nurse practitioner or case manager

8. What is the content of the preoperative physical therapist intervention for patients undergoing major elective abdominal surgery (multiple answers possible)?
- Patient education
- Preoperative assessment of physical functioning
- Exercise prehabilitation

9. Patient education consists of (multiple answers possible)?
- Information leaflet related to physical therapy during hospitalization
- The option to ask questions
- Education about breathing exercises
- Education about circulation exercises
- Other, namely

10. Which components of physical fitness are assessed preoperatively (multiple answers possible)?
- Daily physical activity level
- Peripheral muscle strength
- Inspiratory muscle strength
- Range of motion (passive range of motion, active range of motion)
- Functional mobility (transfers, walking, climbing stairs)
- Cardiorespiratory fitness
- Pain (perceived level of pain during physical activity)
- Cognition
- Fatigue (perceived level of fatigue during physical activity)
- Other, namely

11. Are all patients eligible for exercise prehabilitation?
o Yes
$\circ$ No 


\section{Chapter 2}

12. How do you decide whether a patient is eligible for exercise prehabilitation?

13. What type of physical exercise training is provided during exercise prehabilitation (multiple answers possible)?
○ Inspiratory muscle strength training
o Peripheral muscle strength training
- Cardiorespiratory exercise training
- Functional exercise training
o Other, namely

14. Where does exercise prehabilitation take place (multiple answers possible)?

- At the patient's home

- At a local physical therapy private practice

○ At the hospital

15. Do you make use of any specific performance tests during the preoperative physical therapy visit at your hospital (e.g., ten-meter walk test, steep ramp test, timed up-andgo test)? If yes, please describe them down below.
○ Yes, namely
○ No

16. Do you make use of any specific questionnaires during the preoperative physical therapy visit at your hospital? If yes, please describe them down below.
$\circ$ Yes, namely
○ No

17. How often do you see a patient during the preoperative period?
o Once
- Twice
- Three times
o Four times or more 


\section{Postoperative physical therapist management}

18. Are there any care pathways implemented at your hospital for the postoperative rehabilitation procedure of a patient who underwent major elective abdominal surgery (e.g., "fast-track", "enhanced recovery after surgery", "Better in, Better out")?
○ Yes, namely
$\circ$ No

19. Was the physical therapist involved in the development of this care pathway?
o Yes
$\circ$ No

20. Do all patients who underwent major elective abdominal surgery receive postoperative physical therapy (usual care)?
o Yes
○ No

21. What is the reason that not all patients receive postoperative physical therapy (usual care)?

o It depends on the type of surgery

- It depends on the referral of the ward physician or surgeon

o Other, namely

22. Who is responsible for the involvement of the physical therapy during the postoperative period at your hospital (multiple answers possible)?
$\circ$ The surgeon
- The ward physician
- The nurse
o The physical therapist
- The nurse practitioner or case manager
o Other, namely

23. How many days postoperatively is a patient who underwent major elective abdominal surgery first seen by the physical therapist?
- Postoperative day 0 (at the day of surgery)
- Postoperative day 1 (the first day after surgery)
- Postoperative day 2
- Postoperative day 3
- It depends on a referral for physical therapy
o Other, namely 


\section{Chapter 2}

24. On average, what is the duration of a postoperative physical therapy session? minutes

25. What is the location at which most patients participate in postoperative physical exercise training?
$\circ$ In the patient room at the ward
○ At the department of physical therapy
$\circ$ In a living room
○ Other, namely

26. Can you give a breakdown of percentages concerning the location at which most patients participate in postoperative physical exercise training by shifting the bars (the sum of the three percentages should be 100)?

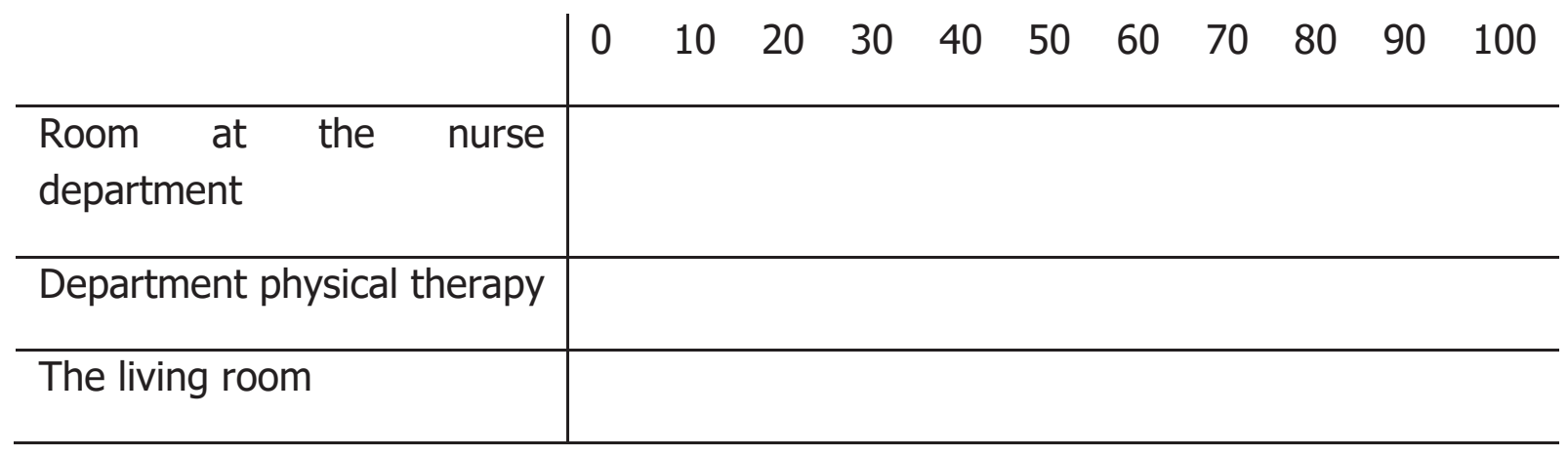

27. How frequently are patients who underwent major elective abdominal surgery seen postoperatively by physical therapy?
- Once a day
- Twice a day
- Three times a day
o Other, namely 
28. Can the physical therapist treatment frequency be adjusted when desired?
$\circ$ Yes
$\circ$ No

29. Are patients who underwent major elective abdominal surgery structurally seen during weekends?
- Yes, on both weekend days
- Yes, only on one weekend day
- Yes, only when there is enough time
- Yes, only in case of postoperative pulmonary complications
$\circ$ No
$\circ$ Other, namely

30. What is the content of postoperative physical therapist interventions for patients who underwent major elective abdominal surgery (multiple answers possible)?
$\circ$ Breathing exercises
- Circulation exercises
- Practicing transfers
- Walking (with or without walking aid)
- Stair climbing
- Peripheral muscle strength exercises
- Cardiorespiratory exercise training
- Patient education related to mobilization during hospital stay
- Patient education homework exercises

31. Are there any other interventions the physical therapist performs postoperatively in daily clinical practice that are not yet mentioned in the previous question?
○ Yes, namely
$\circ$ No

32. Do you (structurally) use validated questionnaires during the clinical postoperative physical therapist treatment of patients who underwent major elective abdominal surgery?
$\circ$ Yes
$\circ$ No 


\section{Chapter 2}

33. Do you (structurally) use validated performance tests during the clinical postoperative physical therapist treatment of patients who underwent major elective abdominal surgery?
○ Yes
○ No

34. Which performance tests do you use postoperatively (multiple answers possible)?

o Six-minute walking test (6MWT)

- Timed up-and-go test (TUG)

- Iowa level of assistance scale (ILOA)

- Modified Iowa level of assistance scale (MILAS)

- De Morton mobility index (DEMMI)

$\circ$ Handgrip strength

o Chair-stand Test

○ Inspiratory muscle strength

o Other, namely

\section{Discharge and readmission}

35. Who decides whether a patient is ready for hospital discharge?

$\circ$ The ward physician

○ The surgeon

○ The physical therapist

o Shared-decision making

36. Are specific discharge criteria used at your hospital for patients who underwent major elective abdominal surgery?
o Yes, namely
$\circ$ No

37. What is the most frequent hospital discharge destination of patients who underwent major elective abdominal surgery at your hospital?
o Home
- Rehabilitation center
○ Nursing home

38. Are you called in consultation on time in case of a readmission of a patient who underwent major elective abdominal surgery?
o Yes
$\circ$ No 
39. Down below you will see two statements. Please, indicate whether these statements apply to your hospital concerning a readmission protocol for patients who underwent major elective abdominal surgery.

\begin{tabular}{llll} 
& Yes & No & Unknown \\
\hline At my hospital, a readmission protocol is available & $\circ$ & $\circ$ & $\circ$ \\
\hline At my hospital, the readmission protocol is followed & $\circ$ & $\circ$ & $\circ$ \\
\hline
\end{tabular}

40. On a scale from 0 to 10 , how useful do you consider a readmission protocol for patients who underwent major elective abdominal surgery?

\begin{tabular}{ccccccccccc}
0 & 1 & 2 & 3 & 4 & 5 & 6 & 7 & 8 & 9 & 10 \\
\hline$\circ$ & $\circ$ & $\circ$ & $\circ$ & $\circ$ & $\circ$ & $\circ$ & $\circ$ & $\circ$ & $\circ$ & $\circ$ \\
\hline
\end{tabular}

41. Are you willing to make your hospital's readmission protocol for patients who underwent major elective abdominal surgery available to us? The document will be analysed anonymously. If yes, please provide us with your e-mail address, so we can contact you.
$\circ$ Yes, my e-mail address is
$\circ$ No
○ Not applicable

This is the end of the e-survey, thank you for your participation. If there are any questions related to this e-survey, please provide them below or send us an e-mail. 


\section{FURTHER CLARIFICATION OF TERMINOLOGY}

- "The option to ask questions" (question 9)

Components of patient education. Was patient education provided in one direction only (providing information to the patient by the physical therapist), or was it also possible for patients to interact with the therapist, to ask questions, and express concerns?

- "Walking" (question 10 and question 30)

Teaching the patient how to ambulate with or without a walking aid as part of the physical therapist intervention postoperatively.

- "Physical fitness, physical activity, cardiorespiratory fitness" (question 10)

Physical activity is any body movement produced by the skeletal muscles that result in an increase in energy expenditure [1]. Here we mean the behavior of individuals that encompasses participating in activities of daily life such as household activities, walking, leisure activities, sport participation, et cetera. Physical fitness is the ability to perform aspects of sports, occupations or daily activities. Cardiorespiratory fitness is a health-related component of physical fitness, and is the maximal capacity body's circulatory and respiratory systems to supply oxygen to skeletal muscles during physical activity/exercise. Cardiorespiratory fitness can directly be measured by a cardiopulmonary exercise test as maximal oxygen consumption $\left(\mathrm{Vo}_{2 \max }\right)$ or can be estimated as the exercise capacity (maximal work rate) from an exercise test.

- "Usual care" (question 20)

Although the definition of usual care has not been standardized, it can include the routine care received by patients for prevention or treatment of diseases [2]. Applied to this survey question it refers to: is it standardized in the route daily care practice that patients receive physical therapy after surgery or is it something that only is giving at a special indication. In other words: does just every patient receive postoperative physical therapy, or is this decided for each individual patient separately? 


\section{REFERENCES SUPPLEMENTARY INFORMATION}

1. Caspersen CJ, Powell KE, Christenson GM, Physical activity, exercise, and physical fitness: definitions and distinctions for health-related research, Public Health Rep. 100 (1985) 126-131.

2. Harlapur M, Shimbo D, Usual care, in: Gellman MD and Turner JR, Encyclopedia of behavioral medicine, Springer New York, New York, 2013, pp. 2021-2022. 



\section{Chapter}

The association between performance parameters of physical fitness and postoperative outcomes in patients undergoing colorectal surgery: an evaluation of care data

Aniek F. Heldens

Bart C. Bongers

Antoine F. Lenssen

Laurents P. Stassen

Wolfgang F. Buhre

Nico L. van Meeteren

European Journal of Surgical Oncology 2017;43:2084-2092. 


\title{
Chapter 3
}

\begin{abstract}
Background.

Preoperative cardiorespiratory fitness, as measured by cardiopulmonary testing or estimated using the less sophisticated incremental shuttle walk test, timed up-and-go test or stair climb test is known to be associated with postoperative outcomes. This study aimed to evaluate whether parameters of physical fitness are associated with postoperative outcomes in patients with colorectal cancer scheduled for elective abdominal resection.
\end{abstract}

\section{Methods.}

Perioperative data of patients who underwent colorectal resection at Maastricht University Medical Center were retrospectively analyzed. Preoperative variables (e.g., age, body mass index, comorbidities, physical fitness, tumor characteristics, neoadjuvant treatment, American Society of Anesthesiologists score, level of perceived fatigue and nutritional status) were compared with postoperative outcomes.

\section{Results.}

Out of 80 consecutive cases, 75 (93.8\%) were available for analysis (57.3\% male, median \pm interquartile range age $69.2 \pm 11.7$ years). A higher Charlson comorbidity index (odds ratio (OR) of 1.604, 95\% confidence interval (CI) 1.120-2.296), worse functional exercise capacity (in meters, OR of $0.995,95 \%$ CI $0.991-1.000$ ), a lower physical activity level (in min/day, OR of $0.994,95 \%$ CI $0.988-1.000$ ), and a higher level of perceived fatigue (OR of 1.047, 95\% CI 1.016-1.078), were associated with a slower time to recovery of physical functioning. A better functional exercise capacity was associated with a lower OR (OR of 0.995 , 95\% CI $0.991-1.000$ ) for non-surgical complications.

\section{Conclusions.}

There is an association between preoperative parameters and postoperative outcomes in patients with colorectal cancer scheduled for abdominal resection. Patients benefit from an optimal preoperative physical fitness level. Specific interventions can target this physical fitness level. 


\section{INTRODUCTION}

In the Netherlands, patients with signs of colorectal cancer are identified via the national screening program for colorectal cancer (see www.bevolkingsonderzoekzuid.nl) or after referral of the general practitioner. After confirmation of the diagnosis, most of the patients (rectal cancer; $99.0 \%$, colon cancer; $87.0 \%$ ) are scheduled for elective colorectal resection [1]. A smaller proportion of patients (rectal cancer; $1.0 \%$, colon cancer; $13.0 \%$ ) requires acute surgery [1]. After the decision for elective surgery, preassessment by the anesthesiologist and the physical therapist for medical conditions and physical fitness is scheduled (see Figure 1 that illustrates the patient journey at the Maastricht University Medical Center).

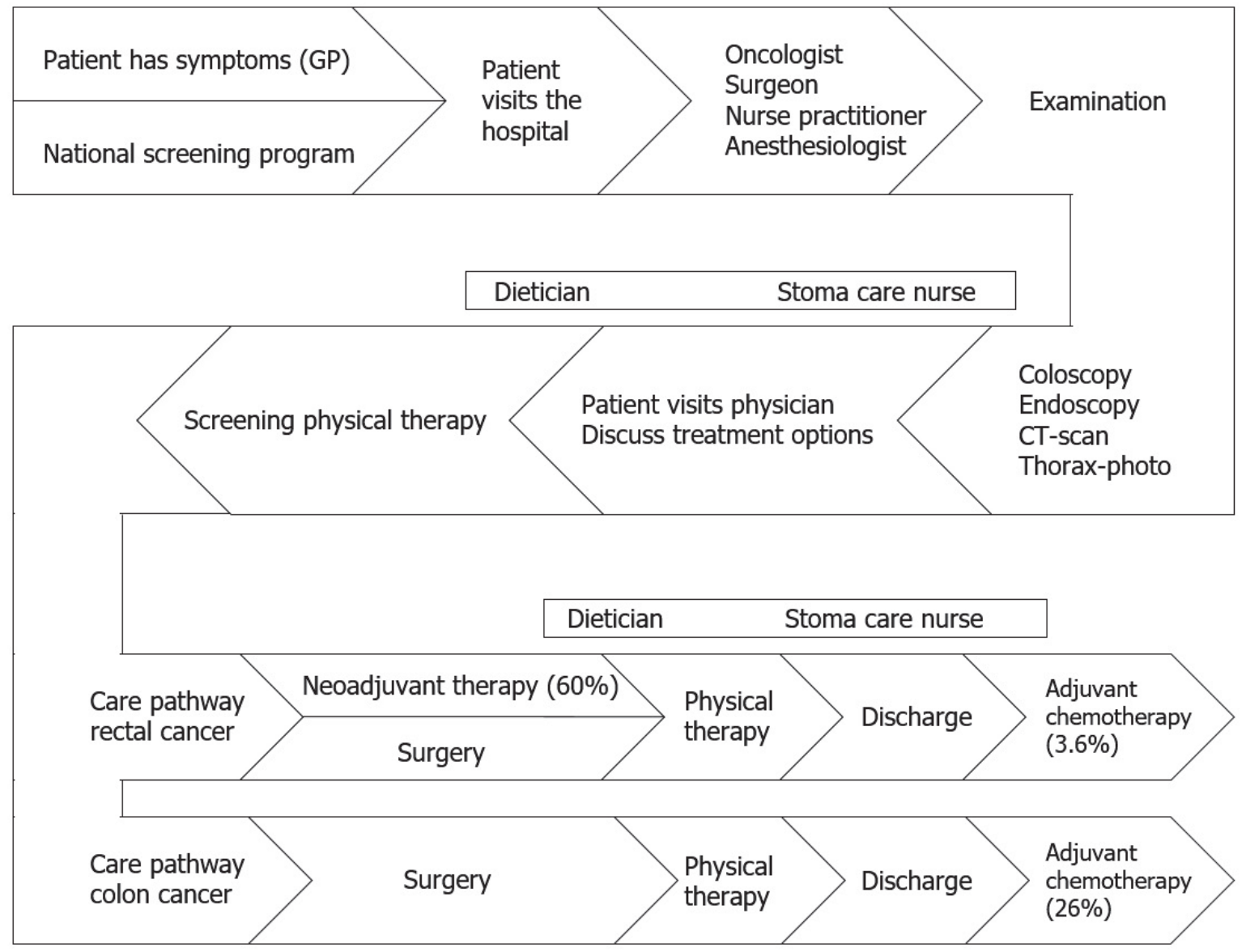

Figure 1. Patient journey for colon and rectal cancer at the Maastricht UMC+. 
Major colorectal surgery is significantly associated with morbidity and even mortality $[2,3]$. Outcome after major surgery depends on several factors such as perioperative care, neoadjuvant treatment, and also physical fitness. Preoperative cardiorespiratory fitness has been reported to have a consistent positive relation with postoperative outcomes in abdominal surgery [4-8]. A poor cardiorespiratory fitness, as measured by a maximal cardiopulmonary exercise test, indicates a reduced physiological reserve, which can contribute to a complicated postoperative time course including morbidity and mortality in patients with lower and upper gastrointestinal cancer, respectively $[5,9]$. Patients with an increased preoperative level of cardiorespiratory fitness may have a greater physiological reserve to better cope with surgical stress that significantly increases metabolic demand after surgery [7].

In most Dutch hospitals, performing a preoperative cardiopulmonary exercise test for risk stratification is not part of standard care for patients undergoing major abdominal surgery. However, performance at less sophisticated physical fitness tests has been found to be related to postoperative outcomes as well. Studies of Nutt et al. [10] and Struthers et al. [11] showed that the incremental shuttle walk test (iSWT) can serve as an indicator of postoperative risk in patients undergoing abdominal surgery. The timedup-and-go test (TUG) [12] is identified as a predictor for postoperative complications in patients undergoing elective surgery (for breast cancer and gastrointestinal malignancies). Other studies identified the stair-climbing test as predictor for postoperative complications in elective abdominal surgery [13] and in surgical resection for non-small cell lung cancer [14]. Alternative physical performance tests that provide information about the patient's physical fitness may therefore also be appropriate tools to perform preoperative risk stratification in several surgical populations.

The aim of this study was therefore to evaluate whether preoperative performance parameters of physical fitness are associated with postoperative outcomes in patients with colorectal cancer scheduled for elective colorectal resection. If specific preoperative parameters are associated with postoperative outcomes, these variables may be targeted with preoperative interventions in order improve postoperative outcomes, trajectories that where already executed by others $[15,16]$.

\section{METHODS}

\section{Participants}

Patients diagnosed with colorectal cancer planned for elective colorectal resection at the Maastricht University Medical Center (Maastricht UMC+) are being monitored pre- and postoperatively for their physical fitness as part of usual care. For this study, data sampling was performed between January 2015 and December 2015. All consecutive 
patients $>18$ years of age who underwent elective colorectal resection for cancer and who had no objection for the use of their usual care data for research purposes were included. When essential data about postoperative outcomes was missing, the data of that patient was excluded from analysis. The medical ethical committee of the Maastricht UMC+ decided (15-4-234) that this study met the ethical policies of the Maastricht UMC+ and the regulations of the Dutch government.

\section{Preoperative screening}

After the decision for elective colorectal resection, patients consulted the hospital physical therapist as soon as possible for a preoperative screening of physical fitness. During the consultation, patients were educated about the importance of adequate physical fitness to better meet the metabolic demands of major surgery. Patients received standard advice about physical activity throughout the preoperative period. Additionally, patients received information about the content and expectations of postoperative physical therapy treatment during hospitalization.

\section{Outcome variables}

Time to recovery of physical functioning (measured by the modified Iowa levels of assistance scale - mILAS [17]) and data on surgical complications (surgical and nonsurgical) were collected from patient files. Time to recovery of physical functioning was defined as the time between the day of surgery and the day at which patients reached a mILAS score of 0 (in days). Data on postoperative complications were registered with the use of the Clavien-Dindo classification [18].

\section{Predictive variables}

Patient characteristics at time of surgery and treatment details were obtained from electronic patient files. The potential predictors age, body mass index, identification via the national screening program or general practitioner, Charlson comorbidity index, tumor, node, metastasis stage, additional neoadjuvant treatment, American Society of Anesthesiologists score, smoking status, nutritional status (short nutritional assessment questionnaire), level of perceived fatigue (multidimensional fatigue index) and operation type were collected. Data from the preoperative screening of physical fitness (functional exercise capacity, muscle strength, functional mobility, and the level of physical activity) were also included.

\section{Functional exercise capacity}

To measure functional exercise capacity, the modified protocol of the iSWT was used [19], with the maximum walking distance as primary outcome measure. The modified protocol of the iSWT is an extension of the original twelve-level iSWT, with the exact same instructions as the original test [20]. The test starts with a walking speed of $1.8 \mathrm{~km} / \mathrm{h}$. 


\section{Chapter 3}

Every level has a duration of one minute and the walking speed increases with $0.6 \mathrm{~km} / \mathrm{h}$ per level. The maximum walking speed is $10.3 \mathrm{~km} / \mathrm{h}$ during level 15, in which subjects are allowed to run. Prior to the walking test, standardized instructions were given and the patients were encouraged to walk as long as possible. Heart rate was monitored during the test. The participants had to move around two markers over a 10-meter course in time with audio signals from a pre-recorded tape. The test was terminated when the patient could not reach the markers on time on two consecutive audio signals. The use of a walking aid, orthopedic shoes or orthoses was permitted. The test was performed under the close surveillance of an experienced hospital physical therapist. The test-retest reliability for the shuttle walk test varies from 0.760 to 0.990 [21].

\section{Muscle strength}

Handgrip strength as measured with a hand held dynamometer (JAMAR $R$ Hydraulic Hand Dynamometer, JAMAR, Patterson Medical Holdings, Inc., Illinois, USA) was measured to provide an indication of the patient's overall muscle strength. Patients were seated in a chair with their elbow flexed at $90^{\circ}$ and the forearm in the neutral position without any arm support from the chair. The patient performed the test three times with the dominant hand, of which the highest value $(\mathrm{kg})$ was reported [22]. Handgrip strength measured with the Jamar dynamometer is a reliable method (intraclass correlation coefficient values $0.85-0.98$ ) [23].

\section{Functional mobility}

Functional mobility was measured with the timed up-and-go test (TUG), in which the time needed to rise from a chair, walk three meters, walk back to the chair, and sit down again, was measured [24]. After one practice trial, the patient was asked to perform the test three times, of which the fastest time (s) was reported.

\section{Level of physical activity}

Patients were asked about the frequency, duration, and intensity of various physical activities (total minutes of walking, cycling, gardening, household activities, and sport activities) during the two weeks before the preoperative screening with the LASA Physical Activity Questionnaire (LAPAQ) [25]. The total amount of physical activity was converted in minutes of physical activity per day (min/day).

\section{Usual care pathway}

Postoperative care was similar for all patients, regardless of the surgical procedure. All patients participated in postoperative physical therapy (started at postoperative day 1), in which recovery of physical functioning was monitored using the mILAS [17]. The mILAS assesses the capability of patients to perform several activities of daily living (transfer from supine position to sitting position and vice versa, transfer from sit-to- 
stand, walking, and stair climbing) and rates the amount of assistance needed. Postoperative physical therapy consisted of airway clearing exercises, strength exercises, practicing transfers, walking, stair climbing (when necessary for independent functioning at home), and improving muscle function and cardiorespiratory capacity.

\section{Data analysis}

The Statistical Package for the Social Sciences for Windows (version 23.0; IBM, SPSS Inc., Chicago, IL, USA) was used for statistical analysis. For all variables, normality was tested with the Kolmogorov-Smirnov test. Data were presented as median and interquartile range (IQR), when the data were not normally distributed. Data on postoperative complications were divided into subgroups (total complications, surgical complications, and non-surgical complications) and dichotomized ( $0=a b s e n t$, $1=$ present). Data on time to recovery of physical functioning was also dichotomized ( $0=$ recovery of physical functioning in $\leq 4$ days, $1=$ recovery of physical functioning in $\geq 5$ days; as the median time to recovery of physical functioning in the patient population of the current study equaled four days). Additionally, potential predictors were added in univariate logistic regression analysis (enter method) to explore the association between individual independent predictors and postoperative outcome variables. Receiver operating characteristic (ROC) analysis was used to establish optimal threshold values of functional exercise capacity as measured with the iSWT distance to predict non-surgical complications. Finally, a multivariate logistic regression analysis was performed to construct a model which explains the variance in outcome variables. To select potential predictor variables for the regression model, a P-value of 0.200 was used. For the other statistical analyses a P-value $<0.05$ was considered statistically significant.

\section{RESULTS}

Preoperative data of 80 patients with colorectal cancer were available for analysis. Of these patients, five $(6.3 \%)$ were excluded because of missing postoperative data (data not available in the database because the nature of the tumor; $n=4$, and missing data about the physical therapy treatment; $n=1)$. This left 75 cases $(93.8 \%)$ for analysis, 43 men (57.3\%) and 32 women (42.7\%) with a median \pm IQR age of $69.2 \pm 11.7$ years. Of these patients, 39 (52.0\%) were identified via the Dutch national screening program for colorectal cancer. These patients had a statistically significant higher muscle strength (39.3 \pm 18.4 vs $30.0 \pm 22.7 \mathrm{~kg} ; P=0.032)$ and a statistically significant lower level of perceived fatigue $(27.0 \pm 20.0$ vs $41.0 \pm 34.8 ; P=0.005)$. There was also a statistically significant difference in clinical tumor stages ( $\mathrm{CT} 1, \mathrm{CN} 1$ and $\mathrm{CN} 2$ ) between these groups. Table 1 shows patient characteristics and baseline values. Treatment details and postoperative outcomes are summarized in Table $2 \mathrm{a}$ and $2 \mathrm{~b}$. 


\section{Chapter 3}

Table 1a. Participant characteristics.

\begin{tabular}{|c|c|c|c|c|c|c|c|}
\hline & \multicolumn{2}{|c|}{$\begin{array}{c}\text { Total } \\
\text { population } \\
(n=75)\end{array}$} & \multicolumn{2}{|c|}{$\begin{array}{c}\text { National } \\
\text { screening } \\
\text { program } \\
(n=39)\end{array}$} & \multicolumn{2}{|c|}{$\begin{array}{c}\text { General } \\
\text { practitioner } \\
(\mathbf{n = 3 6})\end{array}$} & \\
\hline & \multicolumn{2}{|c|}{ Median (IQR) } & \multicolumn{2}{|c|}{ Median (IQR) } & \multicolumn{2}{|c|}{ Median (IQR) } & $P$-value \\
\hline Age (years) & \multicolumn{2}{|c|}{$69.2(11.7)$} & \multicolumn{2}{|c|}{$68.2(10.6)$} & \multicolumn{2}{|c|}{$72.4(19.5)$} & NS \\
\hline BMI $\left(\mathrm{kg} / \mathrm{m}^{2}\right)$ & \multicolumn{2}{|c|}{$26.3(4.6)$} & \multicolumn{2}{|c|}{$26.9(4.8)$} & \multicolumn{2}{|c|}{$25.5(4.1)$} & NS \\
\hline \multicolumn{8}{|l|}{ Preoperative physical fitness } \\
\hline $\begin{array}{l}\text { Functional exercise capacity } \\
\text { (iSWT, m) }\end{array}$ & \multicolumn{2}{|c|}{$530.0(322.5)$} & \multicolumn{2}{|c|}{$530.0(310.0)$} & \multicolumn{2}{|c|}{$560.0(420.0)$} & NS \\
\hline $\begin{array}{l}\text { Muscle strength } \\
\text { (handgrip strength, kg) }\end{array}$ & \multicolumn{2}{|c|}{$34.4(21.3)$} & \multicolumn{2}{|c|}{$39.3(21.4)$} & \multicolumn{2}{|c|}{$30.0(23.0)$} & 0.032 \\
\hline $\begin{array}{l}\text { Functional mobility } \\
\text { (TUG test, s) }\end{array}$ & \multicolumn{2}{|c|}{$5.8(2.0)$} & \multicolumn{2}{|c|}{$5.2(1.6)$} & \multicolumn{2}{|c|}{$6.0(2.7)$} & NS \\
\hline $\begin{array}{l}\text { Level of perceived fatigue } \\
\text { (MFI score) }\end{array}$ & \multicolumn{2}{|c|}{$34.0(26.0)$} & \multicolumn{2}{|c|}{$27.0(20.0)$} & \multicolumn{2}{|c|}{$41.0(25.8)$} & 0.005 \\
\hline $\begin{array}{l}\text { Level of physical activity } \\
\text { (LAPAQ, min/day) }\end{array}$ & \multicolumn{2}{|c|}{$112.2(106.8)$} & \multicolumn{2}{|c|}{$132.8(113.6)$} & \multicolumn{2}{|c|}{$109.3(103.9)$} & NS \\
\hline & $n$ & $\%$ & $n$ & $\%$ & $n$ & $\%$ & \\
\hline Sex (male) & 43 & 57.3 & 25 & 64.1 & 18 & 50.0 & NS \\
\hline Smoking (yes) & 12 & 16.0 & 8 & 20.5 & 4 & 11.1 & NS \\
\hline \multicolumn{8}{|l|}{ Charlson comorbidity index } \\
\hline 0 & 1 & 1.3 & 0 & 0.0 & 1 & 2.8 & NS \\
\hline 1 & 7 & 9.3 & 0 & 0.0 & 7 & 19.4 & NS \\
\hline 2 & 18 & 24.0 & 15 & 38.5 & 3 & 8.3 & NS \\
\hline 3 & 24 & 32.0 & 16 & 41.0 & 8 & 22.2 & NS \\
\hline 4 & 14 & 18.7 & 6 & 15.4 & 8 & 22.2 & NS \\
\hline 5 & 5 & 6.7 & 1 & 2.6 & 4 & 11.1 & NS \\
\hline 6 & 2 & 2.7 & 0 & 0.0 & 2 & 5.6 & NS \\
\hline 7 & 3 & 4.0 & 1 & 2.6 & 2 & 5.6 & NS \\
\hline 8 & 1 & 1.3 & 0 & 0.0 & 1 & 2.8 & NS \\
\hline
\end{tabular}

Abbreviations: $B M I=B o d y$ mass index $(\mathrm{kg} / \mathrm{m} 2)$; IQR=interquartile range; iSWT=incremental shuttle walk test; LAPAQ=LASA physical activity questionnaire; MFI=Multidimensional fatigue index; $N S=$ not statistically significant; TUG=timed up-and-go. 
Table 1b. Participant characteristics.

\begin{tabular}{|c|c|c|c|c|c|c|c|}
\hline & \multicolumn{2}{|c|}{$\begin{array}{c}\text { Total } \\
\text { population } \\
(n=75)\end{array}$} & \multicolumn{2}{|c|}{$\begin{array}{l}\text { National } \\
\text { screening } \\
\text { program } \\
(n=39)\end{array}$} & \multicolumn{2}{|c|}{$\begin{array}{c}\text { General } \\
\text { practitioner } \\
(\mathbf{n}=\mathbf{3 6})\end{array}$} & \\
\hline & $n$ & $\%$ & $n$ & $\%$ & $n$ & $\%$ & \\
\hline \multicolumn{8}{|c|}{ Clinical TNM stage } \\
\hline $\mathrm{T} \quad \mathrm{cT} 1$ & 16 & 21.3 & 14 & 35.9 & 2 & 5.6 & 0.003 \\
\hline $\mathrm{cT} 2$ & 10 & 13.3 & 5 & 12.8 & 5 & 13.9 & NS \\
\hline cT3 & 27 & 36.0 & 13 & 33.3 & 14 & 38.9 & NS \\
\hline cT4 & 10 & 13.3 & 3 & 7.7 & 7 & 19.4 & NS \\
\hline cTx & 12 & 16.0 & 4 & 10.2 & 8 & 22.2 & NS \\
\hline $\mathrm{N} \quad \mathrm{cNO}$ & 36 & 48.0 & 20 & 51.3 & 16 & 44.4 & NS \\
\hline $\mathrm{cN} 1$ & 13 & 17.3 & 11 & 28.2 & 2 & 5.6 & 0.014 \\
\hline cN2 & 18 & 24.0 & 5 & 12.8 & 13 & 36.1 & 0.010 \\
\hline $\mathrm{cNx}$ & 8 & 10.7 & 3 & 7.7 & 5 & 13.9 & NS \\
\hline M $\quad \mathrm{cMO}$ & 72 & 96.0 & 38 & 97.4 & 34 & 94.4 & NS \\
\hline $\mathrm{cM} 1$ & 2 & 2.7 & 1 & 2.6 & 1 & 2.8 & NS \\
\hline cMx & 1 & 1.3 & 0 & 0.0 & 1 & 2.8 & NS \\
\hline
\end{tabular}

Abbreviations: NS=not statistically significant; TNM=Tumor, Node, Metastasis. 


\section{Chapter 3}

Table 2a. Participant treatment details.

\begin{tabular}{|c|c|c|c|c|c|c|c|}
\hline & \multicolumn{2}{|c|}{$\begin{array}{c}\text { Total } \\
\text { population } \\
(n=75)\end{array}$} & \multicolumn{2}{|c|}{$\begin{array}{l}\text { Identification } \\
\text { via national } \\
\text { screening } \\
\text { program } \\
(n=39)\end{array}$} & \multicolumn{2}{|c|}{$\begin{array}{l}\text { Identification } \\
\text { via general } \\
\text { practitioner } \\
\quad(n=36)\end{array}$} & \multirow[b]{2}{*}{$P$-value } \\
\hline & $n$ & $\%$ & $n$ & $\%$ & $n$ & $\%$ & \\
\hline \multicolumn{8}{|l|}{ Tumor distance from anal verge } \\
\hline$<5.0 \mathrm{~cm}$ & 5 & 6.7 & 1 & 2.6 & 4 & 11.1 & NS \\
\hline $5.1-10.0 \mathrm{~cm}$ & 15 & 20.0 & 5 & 12.8 & 10 & 27.8 & NS \\
\hline$>10.1 \mathrm{~cm}$ & 55 & 73.3 & 33 & 84.6 & 22 & 61.1 & NS \\
\hline \multicolumn{8}{|l|}{ Operation type } \\
\hline Transabdominal laparoscopic & 61 & 81.3 & 35 & 89.7 & 26 & 72.2 & NS \\
\hline Transabdominal open & 14 & 18.7 & 4 & 10.3 & 10 & 27.8 & NS \\
\hline \multicolumn{8}{|l|}{ Surgery } \\
\hline Extended right hemicolectomy & 19 & 25.3 & 8 & 20.5 & 10 & 27.8 & NS \\
\hline Transversum resection & 1 & 1.3 & 0 & 0.0 & 1 & 2.8 & NS \\
\hline Extended left hemicolectomy & 6 & 8.0 & 5 & 12.8 & 1 & 2.8 & NS \\
\hline Low anterior resection & 44 & 58.7 & 24 & 61.5 & 21 & 58.3 & NS \\
\hline Abdomino-perianal resection & 5 & 6.7 & 2 & 5.1 & 3 & 8.3 & NS \\
\hline \multicolumn{8}{|l|}{ Pathological TNM stage } \\
\hline $\mathrm{T} \quad \mathrm{pT} 0$ & 0 & 0.0 & 0 & 0.0 & 0 & 0.0 & NS \\
\hline pT1 & 22 & 29.3 & 18 & 46.2 & 4 & 11.1 & NS \\
\hline pT2 & 21 & 28.0 & 9 & 23.1 & 12 & 33.3 & NS \\
\hline pT3 & 27 & 36.0 & 11 & 28.2 & 16 & 44.4 & NS \\
\hline pT4 & 5 & 6.7 & 1 & 2.6 & 4 & 11.1 & NS \\
\hline $\mathrm{N} \quad \mathrm{pNO}$ & 49 & 65.3 & 24 & 61.5 & 27 & 75.0 & NS \\
\hline $\mathrm{pN} 1$ & 23 & 30.7 & 15 & 38.5 & 8 & 22.2 & NS \\
\hline $\mathrm{pN} 2$ & 3 & 4.0 & 0 & 0.0 & 3 & 8.3 & NS \\
\hline M $\mathrm{pMO}$ & 68 & 90.7 & 38 & 97.4 & 30 & 83.3 & NS \\
\hline pM1 & 3 & 4.0 & 1 & 2.6 & 2 & 5.6 & NS \\
\hline $\mathrm{pMx}$ & 4 & 5.3 & 0 & 0.0 & 4 & 13.9 & NS \\
\hline \multicolumn{8}{|l|}{ Neoadjuvant radiation } \\
\hline Chemoradiation & 15 & 20.0 & 4 & 10.3 & 11 & 15.9 & NS \\
\hline Short term radiation & 3 & 4.0 & 2 & 5.1 & 1 & 2.8 & NS \\
\hline Neoadjuvant chemotherapy & 10 & 13.3 & 3 & 7.7 & 7 & 19.4 & NS \\
\hline \multicolumn{8}{|l|}{ ASA score } \\
\hline I & 13 & 17.3 & 8 & 20.5 & 5 & 13.9 & NS \\
\hline II & 46 & 61.3 & 25 & 64.1 & 21 & 58.3 & NS \\
\hline III & 16 & 21.3 & 6 & 15.4 & 10 & 27.8 & NS \\
\hline
\end{tabular}

Abbreviations: $A S A=A m e r i c a n$ Society of Anaesthesiologists score; NS=not statistically significant; TNM=Tumor, Node, Metastasis. 
Table 2b. Postoperative outcomes.

\begin{tabular}{|c|c|c|c|c|c|c|c|}
\hline & \multicolumn{2}{|c|}{$\begin{array}{c}\text { Total } \\
\text { population } \\
(n=75)\end{array}$} & \multicolumn{2}{|c|}{$\begin{array}{c}\text { Identification } \\
\text { Via national } \\
\text { screening } \\
\text { program } \\
(n=39)\end{array}$} & \multicolumn{2}{|c|}{$\begin{array}{l}\text { Identification } \\
\text { via general } \\
\text { practitioner } \\
\quad(n=36)\end{array}$} & \multirow[b]{2}{*}{$P$-value } \\
\hline & $n$ & $\%$ & $n$ & $\%$ & $n$ & $\%$ & \\
\hline \multicolumn{8}{|l|}{ Postoperative outcomes } \\
\hline Complications & 27 & 36.0 & 12 & 30.8 & 15 & 41.7 & NS \\
\hline Readmission & 8 & 10.7 & 3 & 7.7 & 5 & 13.9 & NS \\
\hline Mortality & 2 & 2.7 & 1 & 2.6 & 1 & 2.8 & NS \\
\hline & \multicolumn{2}{|c|}{ Median (IQR) } & \multicolumn{2}{|c|}{ Median (IQR) } & \multicolumn{2}{|c|}{ Median (IQR) } & P-value \\
\hline Length of stay (days) & \multicolumn{2}{|c|}{$6.0(6.0)$} & \multicolumn{2}{|c|}{$5.0(3.0)$} & \multicolumn{2}{|c|}{$7.5(6.8)$} & 0.009 \\
\hline $\begin{array}{l}\text { Time to recovery of physical } \\
\text { functioning (days) }\end{array}$ & \multicolumn{2}{|c|}{$4.0(3.0)$} & \multicolumn{2}{|c|}{$4.0(2.0)$} & \multicolumn{2}{|c|}{$5.5(5.8)$} & 0.011 \\
\hline
\end{tabular}

Abbreviations: IQR=interquartile range; $N S=$ not statistically significant.

\section{Postoperative complications}

Postoperatively, 27 complications (36.0\%) were registered, of which $20(74.1 \%)$ were surgical (e.g., anastomotic leakage) and seven (25.9\%) were non-surgical (e.g., pulmonary complications). Five patients (6.7\%) suffered from anastomotic leakage, of which one patient received preoperative radiation (in combination with chemotherapy) and two patients had preoperative anemia. Redo-surgery was necessary in 9 of the 20 patients with surgical complications (45.0\%). According to the Clavien-Dindo classification there were sixteen (59.3\%) grade I, one (3.7\%) grade II, seven $(25.9 \%)$ grade III, one (3.7\%) grade IV and two (7.4\%) grade V complications. The latter two patients died at the hospital due to complications following redo-surgery for anastomotic leakage (overall mortality rate of $2.7 \%$ ). Eight (10.7\%) readmissions were reported. 


\section{Chapter 3}

\section{Time to recovery of physical functioning}

Median \pm IQR time to recovery of physical functioning was $4.0 \pm 3.0$ days. When data were clustered according to type of surgical procedure, transabdominal laparoscopic surgery $(n=61)$ versus transabdominal open surgery $(n=14)$, a statistically significant difference in time to recovery of physical functioning ( $4.0 \pm 2.0$ versus $8.0 \pm 8.25$ days; $P<0.001$ ) was observed. Additionally, patients that were identified via the national screening program had a statistically significant faster time to recovery of physical functioning ( $4.0 \pm 2.0$ vs $5.5 \pm 5.8$ days; $P=0.011$ ). A separate analysis showed no difference in time to recovery of physical functioning between laparoscopic and open surgery in the group of patients that was identified via the national screening program. In the group of patients that was identified via the general practitioner there was a statistically significant difference found between laparoscopic and open surgery (time to recovery of physical functioning of $10.0 \pm 8.5$ vs $4.0 \pm 3.0$ days; $P=0.006$ ).

Results of the univariate logistic regression are shown in Table 3. Logistic regression analysis showed that functional exercise capacity, nutritional status, level of perceived fatigue score and, muscle strength were independent predictors of non-surgical complications. The model with functional exercise capacity predicted $14.8 \%$ of the variance in non-surgical complications (pseudo $\mathrm{R}^{2}$ ). A higher functional exercise capacity and higher muscle strength were associated with smaller odds ratio's (ORs) for nonsurgical complications. If the walking distance on the iSWT increased by one meter, such as from 175 to 176 meter or from 250 to 251 meter, the odds of experiencing nonsurgical complications equals 0.995 . For a 50 -meter difference in walking distance on the iSWT, the odds ratio is $e^{(50 \times-0.005)}=0.78$, whereas for a 100 -meter difference in walking distance (the example mentioned in the manuscript), the odds ratio is $e^{(100 \times-0.005)}=$ 0.61. Patients with a worse nutritional status and a higher level of perceived fatigue were more likely to experience non-surgical complications. ROC analysis depicted in Figure 2 shows the value of the iSWT to predict non-surgical complications. The area under the curve (95\% confidence interval) was $0.755(0.592-0.918, P=0.027)$. 


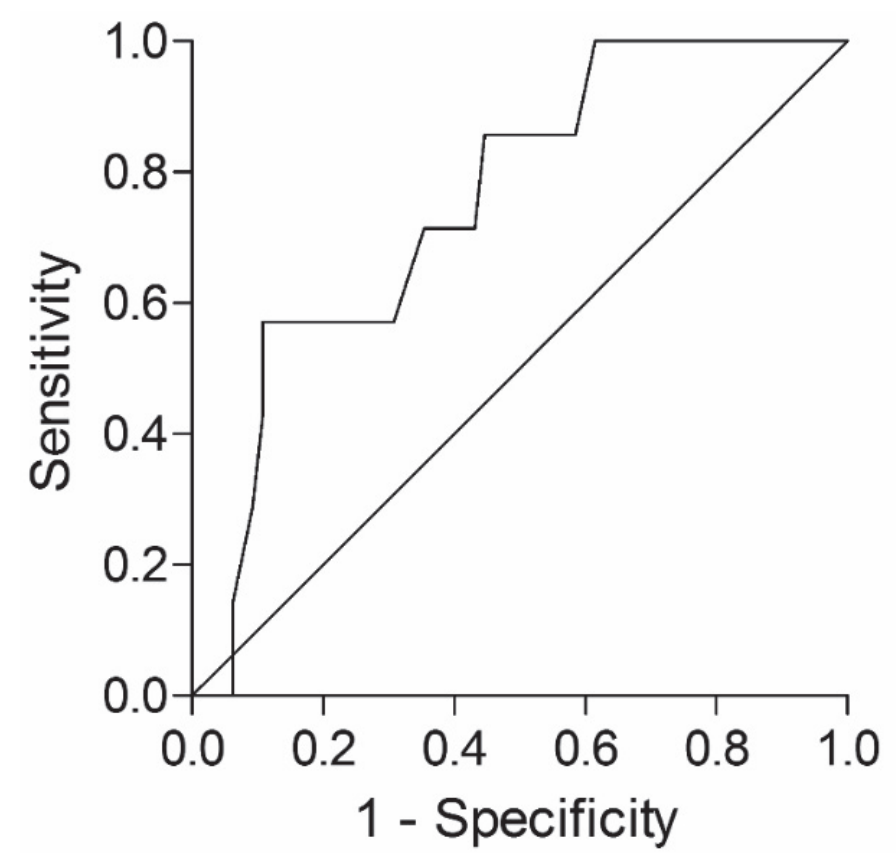

Figure 2. ROC analysis for predicting postoperative complications from iSWT distance. Abbreviations: iSWT=incremental shuttle walk test; ROC=receiver operating characteristic.

Further analysis showed that the Charlson comorbidity index, identification via the national screening program, age, functional exercise capacity, functional mobility, level of perceived fatigue, and level of physical activity were independent predictors of a longer time to recovery of physical functioning. The OR for functional mobility was 1.274 , which indicates that each one-second increase in TUG outcome increased the odds of having a prolonged time to recovery of physical functioning by 0.274 . The model with the level of perceived fatigue had a pseudo $\mathrm{R}^{2}$ of $19.3 \%$. The American Society of Anesthesiologists score was not related to postoperative outcomes. 


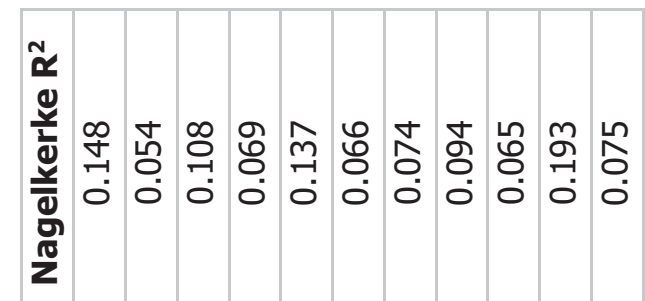

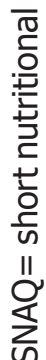

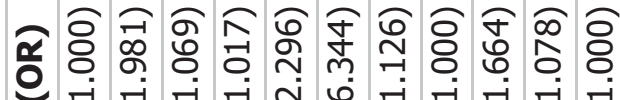

- 11 d ó

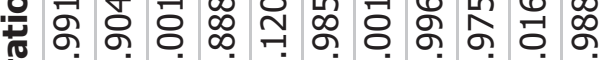

-

ํㅠㅇ ભ

Оั

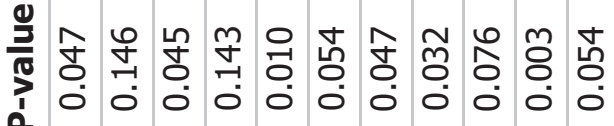

흔

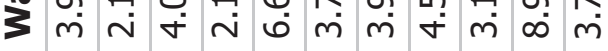

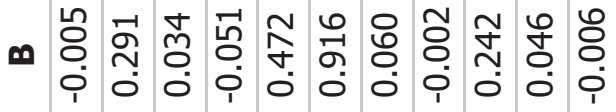

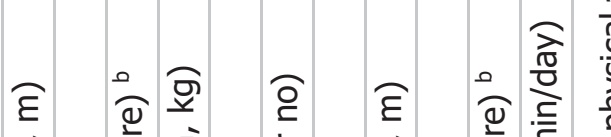

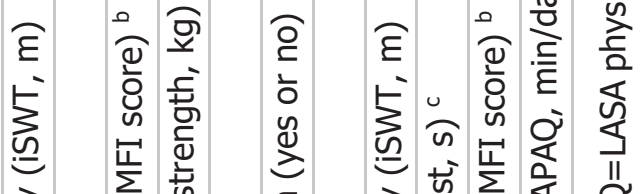

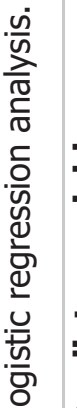

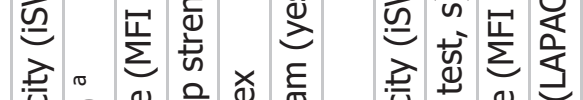

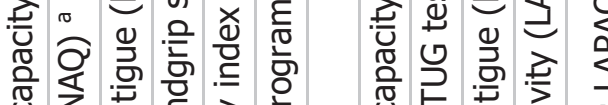

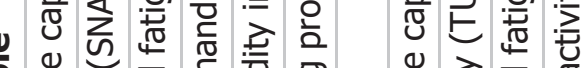

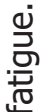

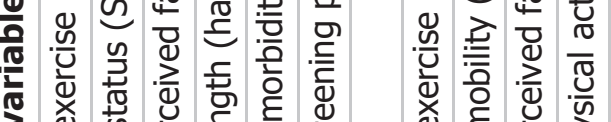

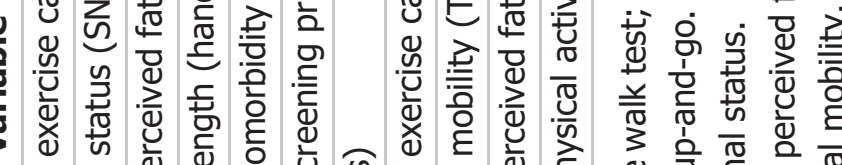

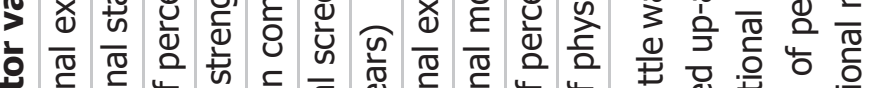

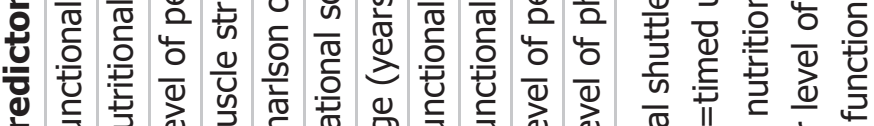

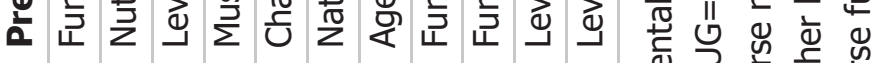

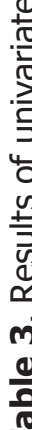

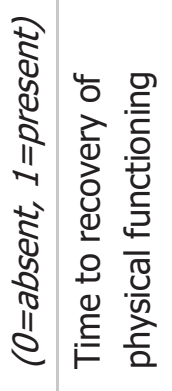

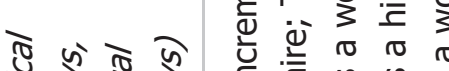

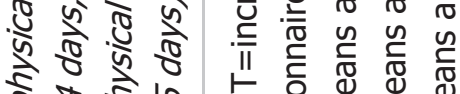

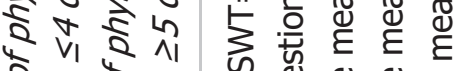

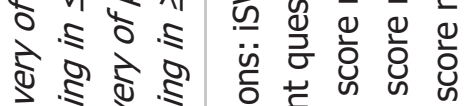

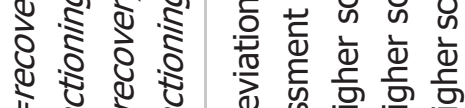

iा

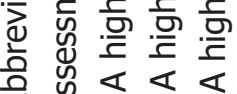


Multivariate logistic regression analysis gave additional value in the prediction of postoperative time to recovery of physical functioning. The level of perceived fatigue (OR of $1.044,95 \%$ confidence interval (CI) of 1.013-1.076) and physical activity level (OR of $0.995,95 \%$ CI of $0.989-1.002$ ) were associated with $22.8 \%$ of the variance in time to recovery of physical functioning. Patients with a higher level of perceived fatigue and a lower level of preoperative physical activity were more likely to have a longer time to recovery of physical functioning. Surgical complications and readmissions could not be predicted with the current data set and statistical analyses.

\section{DISCUSSION}

The aim of this study was to evaluate the associations between preoperative parameters of physical fitness and postoperative outcomes in patients with colorectal cancer scheduled for elective colorectal resection. The results of this study showed that several preoperative parameters were associated with the patient's postoperative outcomes. Functional exercise capacity, functional mobility, level of perceived fatigue, and level of physical activity were independent predictors of a shorter postoperative time to recovery of physical functioning. Preoperative physical functioning (functional exercise capacity, muscle strength, level of perceived fatigue) and nutritional status were associated with the presence of non-surgical complications. Patients with a better preoperative level of physical fitness are less likely to experience non-surgical complications. In addition, patients with a worse preoperative nutritional status were more likely to experience nonsurgical complications.

A ROC analysis of our data showed a value of $290 \mathrm{~m}$ on iSWT as the most optimal threshold to predict non-surgical complications with a sensitivity of 0.570 and a specificity of 0.890 . Previous literature on the iSWT shows a threshold of $360 \mathrm{~m}$ [11] and $250 \mathrm{~m}$ [10], respectively to identify patients at risk for postoperative complications in abdominal surgery. A higher sensitivity at the ROC analysis (which is often more desirable in daily practice) provides a value of $515 \mathrm{~m}$ on iSWT as threshold to predict non-surgical complications with a sensitivity of 0.857 and a specificity of 0.554 . Consequently, postoperative outcomes were better in patients with a preoperative walking distance of $515 \mathrm{~m}$ or higher.

The presence of comorbidities, identification via the general practitioner, a higher age, a lower functional exercise capacity, a lower functional mobility and physical activity level, and a higher level of perceived fatigue were associated with a longer time to recovery of physical functioning $(P<0.200)$. Previous literature already showed the role of preoperative physical fitness and physical activity and its relation with postoperative outcomes $[26,27]$. Feeney et al. showed that patients who developed a postoperative pulmonary complication after an esophagectomy had a lower preoperative physical 
activity level (20 $\pm 13.7 \mathrm{~min} /$ day versus $36 \pm 20.7 \mathrm{~min} /$ day, $P=0.010)$ [26]. The study of Dronkers et al. showed an independent association between adequate preoperative physical activity level (OR of 5.5, 95\% confidence interval of 1.400-21.900) and shortterm mortality [27].

Our results showed a statistically significant shorter time to recovery of physical functioning for patients that were identified via the national screening program and patients that underwent laparoscopic surgery. Previous literature comparing laparoscopic surgery with open surgery also showed statistically significant differences in length of stay and other postoperative outcomes, with a higher overall morbidity rate, more complications and a higher mortality rate in patients that underwent open surgery $[28,29]$. Patients identified via the national screening program (early inclusion) tended to be younger and fitter. Thereby, these patients had less disease-related complaints and a better TNM classification, because of the early stage of the disease. The earlier disease stage increases the options for a laparoscopic procedure, which is usually less invasive and associated with a faster medical recovery and recovery of physical functioning $[28,29]$. In our patient population, the percentage of laparoscopic procedures was $89.7 \%$ in the group of patients that was identified via the national screening program (versus $72.2 \%$ in the group identified via the general practitioner).

\section{Lessons learned}

This study focused on patients scheduled for colorectal resection, but the used methods could probably be translated to other surgical populations as well. Our data showed statistically significant associations between functional exercise capacity, Charlson comorbidity index, level of perceived fatigue, and level of physical activity, and postoperative outcomes; however, the data could not be used to develop a clear prediction rule that can sufficiently explain the variance in postoperative outcomes. A larger patient population is necessary to make a valid prediction model and this study may be seen as the groundwork hereto. A multicenter study with four hospitals has recently started in the Netherlands.

Additional analysis was performed to explore the association between individual independent variables and desirable postoperative outcomes (no complications and a shorter time to recovery of physical functioning). Analysis showed that a higher body mass index, identification via the national screening program, and a higher preoperative physical activity level were associated with no occurrence of surgical complications. A higher functional exercise capacity was associated with no occurrence of non-surgical complications. A laparoscopic procedure, a lower preoperative perceived level of fatigue and a higher preoperative level of physical activity were associated with a shorter time to recovery of physical functioning ( $\leq 4$ days). 
In conclusion, the current study revealed associations between preoperative physical fitness and postoperative outcomes in patients with colorectal cancer scheduled for abdominal resection. A complete preoperative evaluation is valuable for patients and their caregivers and can ensure a quick start for appropriate preoperative interventions. Finally, it seems of interest to explore which (preoperative) variables can predict desirable postoperative outcomes. If preoperative parameters can distinguish patients not at risk from the total population, this group can be in- and/or excluded from additional preoperative interventions. 


\section{REFERENCES}

1. Dutch Institute for Clinical Auditing (DICA) Leiden, DICA Jaarrapportage 2015: Dutch surgical colorectal audit (DSCA). http://www.clinicalaudit.nl/jaar-rapportage/2015/dsca.html, 2016 (accessed November 2016).

2. Andreoni B, Chiappa A, Bertani E, et al., Surgical outcomes for colon and rectal cancer over a decade: results from a consecutive monocentric experience in 902 unselected patients, World J Surg Oncol. 5 (2007) 73.

3. Burns EM, Bottle $A$, Aylin $P$, et al., Variation in reoperation after colorectal surgery in England as an indicator of surgical performance: retrospective analysis of Hospital Episode Statistics, Br Med J. 343 (2011) d4836.

4. Snowden $\mathrm{CP}$, Prentis J, Jacques $\mathrm{B}$, et al., Cardiorespiratory fitness predicts mortality and hospital length of stay after major elective surgery in older people, Ann Surg. 257 (2013) 999-1004.

5. West MA, Lythgoe $\mathrm{D}$, Barben $\mathrm{CP}$, et al., Cardiopulmonary exercise variables are associated with postoperative morbidity after major colonic surgery: a prospective blinded observational study, $\mathrm{Br}]$ Anaesth. 112 (2014) 665-671.

6. West MA, Parry MG, Lythgoe $D$, et al., Cardiopulmonary exercise testing for the prediction of morbidity risk after rectal cancer surgery, $\mathrm{Br}$ J Surg. 101 (2014) 1166-1172.

7. Levett $D Z$, Grocott MP, Cardiopulmonary exercise testing for risk prediction in major abdominal surgery, Anesthesiol Clin. 33 (2015) 1-16.

8. Hennis PJ, Meale PM, Grocott MP, Cardiopulmonary exercise testing for the evaluation of perioperative risk in non-cardiopulmonary surgery, Postgrad Med J. 87 (2011) 550-557.

9. Jack $S$, West MA, Raw $D$, et al., The effect of neoadjuvant chemotherapy on physical fitness and survival in patients undergoing oesophagogastric cancer surgery, Eur J Surg Oncol. 40 (2014) 13131320.

10. Nutt CL, Russell JC, Use of the pre-operative shuttle walk test to predict morbidity and mortality after elective major colorectal surgery, Anaesthesia. 67 (2012) 839-849.

11. Struthers R, Erasmus $\mathrm{P}$, Holmes $\mathrm{K}$, et al., Assessing fitness for surgery: a comparison of questionnaire, incremental shuttle walk, and cardiopulmonary exercise testing in general surgical patients, $\mathrm{Br}$ J Anaesth. 101 (2008) 774-780.

12. Huisman MG, van Leeuwen BL, Ugolini G, et al., "Timed Up \& Go": a screening tool for predicting 30day morbidity in onco-geriatric surgical patients? A multicenter cohort study, PLoS One. 9 (2014) e86863.

13. Reddy S, Contreras CM, Singletary B, et al., Timed stair climbing is the single strongest predictor of perioperative complications in patients undergoing abdominal surgery, J Am Coll Surg. 222 (2016) 559-566.

14. Dong J, Mao Y, Li J, et al., Stair-Climbing test predicts postoperative cardiopulmonary complications and hospital stay in patients with non-small cell lung cancer, Med Sci Monit. 23 (2017) 1436-1441.

15. Hoogeboom TJ, Dronkers JJ, Hulzebos EH, et al., Merits of exercise therapy before and after major surgery, Curr Opin Anaesthesiol. 27 (2014) 161-6.

16. Hulzebos EH, van Meeteren NL, Making the elderly fit for surgery, Br J Surg. 103 (2016) 12-15.

17. Shields RK, Enloe L, Evans RE, et al., Reliability, validity, and responsivesness of functional tests in patients with total joint replacement, Phys Ther. 75 (1995) 176-179.

18. Dindo D, Demartines N, Clavien PA, Classification of surgical complications: a new proposal with the evaluation in a cohort of 6336 patients and results of a survey, Ann Surg. 240 (2004) 205-213.

19. Bradley J, Howard J, Wallace E, et al., Validity of a modified shuttle walk test in adult cystic fibrosis, Thorax. 54 (1999) 437-439. 
20. Singh SJ, Morgan MD, Scott S, et al., Development of a shuttle walking test of disability in patients with chronin airways obstruction, Thorax. 47 (1992) 1019-1024.

21. Parreira VF, Janaudis-Ferreira T, Evans RA, et al., Measurement properties of the incremental shuttle walk test, A systematic review, Chest. 145 (2014) 1357-1369.

22. Fess E, Grip strength, in: American society of hand therapists, clinical assessment recommendations, $2^{\text {nd }}$ edition, The Society, Chicago, 1992, pp. 41-45.

23. Peolsson A, Hedlund $R$, Oberg $B$, Intra- and inter-tester reliability and reference values for hand strength, J Rehabil Med. 33 (2001) 36-41.

24. Gautschi OP, Carniola MV, Joshing $\mathrm{H}$, et al., The timed up and go test for lumbar degenerative disc disease, J Clin Neurosci. 22 (2015) 1943-1948.

25. Steel VS, Smit JH, Plumb SM, et al., Comparison of the LASA physical activity questionnaire with a 7day diary and pedometer, J Clin Epidemiol. 57 (2004) 252-258.

26. Feeney C, Reynolds JV, Hussey J, Preoperative physical activity levels and postoperative pulmonary complications post-esophagostomy, Dis Oesophagus. 24 (2011) 489-494.

27. Dronkers JJ, Chorus AM, van Meeteren NL, et al., The association of pre-operative physical fitness and physical activity with outcome after scheduled major abdominal surgery, Anaesthesia. 68 (2013) 67-73.

28. Vallribera Valls F, Landi F, Espín Basany E, et al., Laparoscopy-assisted versus open colectomy for treatment of colon cancer in the elderly: morbidity and mortality outcomes in 545 patients, Surg Endosc. 28 (2014) 3373-3378.

29. Kennedy GD, Rajamanickam V, O'connor ES, et al., Optimizing surgical care of colon cancer in the older adult population, Ann Surg. 253 (2011) 508-514. 


\section{SUPPLEMENTARY INFORMATION}

Table S1. The modified Iowa levels of assistance scale (mILAS).

\begin{tabular}{|l|l|}
\hline Modified Iowa levels of assistance scale (mILAS) & Score \\
\hline Frtivity/task & \\
\hline From lying to sitting & \\
\hline From sit to stand & \\
\hline Walking & \\
\hline Climbing stairs & \\
\hline & \\
\hline Total score & \\
\hline
\end{tabular}

\begin{tabular}{|l|l|l|}
\hline Score & Assistance & $\begin{array}{l}\text { Description } \\
\text { No assistance necessary to perform the task safely } \\
\text { (with or without walking aid) }\end{array}$ \\
\hline 0 & $\begin{array}{l}\text { Dependent } \\
\text { Only supervision necessary to perform the task safely }\end{array}$ \\
\hline 1 & $\begin{array}{l}\text { Stand by } \\
\text { Minimal }\end{array}$ & $\begin{array}{l}\text { 2 point of contact necessary to perform the task safely } \\
\text { (1 or } 2 \text { people) }\end{array}$ \\
\hline 3 & Moderate & 3 or more points of contact necessary to perform the task safely \\
\hline 4 & A lot & Not able to perform (even with a maximum of assistance \\
\hline 5 & Unachievable & Actitvity was not performed/tested \\
\hline 6 & Not tested & \\
\hline
\end{tabular}






\section{Chapter}

\section{Clinical course of physical fitness and skeletal muscle mass during neoadjuvant chemoradiotherapy in patients with locally advanced rectal cancer: an observational longitudinal study of 25 single subjects}

Aniek F. Heldens

Bart C. Bongers

Judith de Vos-Geelen

Iris J. Minis-Rutten

Laurents P. Stassen

Wolfgang F. Buhre

Nico L. van Meeteren

Submitted for publication. 


\section{Chapter 4}

\section{ABSTRACT}

\section{Background.}

Patients with locally advanced rectal cancer are often considered for neoadjuvant chemoradiotherapy ( $\mathrm{nCRT}$ ) before resection. nCRT can have negative effects on physical fitness and skeletal muscle mass, which can negatively influence clinical outcomes. The aim of this study was to evaluate physical fitness and skeletal muscle mass before and after nCRT in single subjects with locally advanced rectal cancer.

\section{Methods.}

Routine care data were retrospectively analyzed. Data consisted of tumor characteristics, clinical data (e.g., side effects and toxicity of the nCRT, loss of body mass), data on performance-based physical fitness (aerobic capacity, muscle strength, and functional mobility), and computed tomography-derived skeletal muscle mass, measured before and after nCRT.

\section{Results.}

Data from 25 single subjects were available. Aerobic capacity $(n=25, P=0.033)$ and skeletal muscle mass $(n=16, P=0.005)$ were significantly reduced after $n C R T$. Although not statistically significant, the largest part of the patients demonstrated a decrease in muscle strength and functional mobility after completing nCRT. In fourteen patients (56\%) adverse events, dose-limiting toxicity or early termination of treatment occurred. Patients who decreased in physical fitness following nCRT seem to experience these negative side effects more often.

\section{Conclusions.}

Aerobic capacity and skeletal muscle mass decreased following nCRT; however, large inter-individual differences were observed concerning the changes in physical fitness, muscle mass and the experience of negative side effects during nCRT. These differences require a personalized approach for each patient during the treatment course. 


\section{INTRODUCTION}

Colorectal cancer is the third most common type of cancer in the Netherlands [1], with a total incidence of 13,739 in 2017, including 3,715 new cases (27.0\%) of rectal cancer [1]. Patients with established high risk based on resectability and locally advanced rectal cancer (tumor, node, metastasis (TNM) stage CT4 or cT3 with a distance to the mesorectal fascia $\leq 1 \mathrm{~mm}$ and/or cN2 or extramesorectal pathological nodes) [2] are considered for an extensive treatment protocol of neoadjuvant chemoradiotherapy (nCRT) [3-7]. In 2017, 48.0\% of patients with rectal cancer in the Netherlands received neoadjuvant therapy (radiotherapy and/or chemotherapy) prior to elective surgery, of which $26.2 \%$ received a combination of both [8]. nCRT aims to control local disease and improve surgical resectability by downsizing the tumor $[6,9]$. However, chemoradiotherapy is a therapy with significant toxic side effects, which can lead to comorbidities like extensive diarrhea, hand-foot syndrome, cardiotoxicity, and anorexia [10]. Additionally, chemoradiotherapy can have negative physical side effects, as fatigue [11] and a decrease in aerobic capacity [3-5,12].

A consistent positive relation has been reported between preoperative aerobic capacity and postoperative outcomes following major abdominal surgery [4,13-17]. Furthermore, literature shows an independent association between low skeletal muscle mass and a poor overall survival after resection for advanced rectal cancer [18]. Table 1 summarizes previous studies that investigated the impact of neoadjuvant therapy on physical fitness [3-5] and skeletal muscle mass [19-22] in different patient populations. 
Table 1. Summary of previous literature investigating the impact of neoadjuvant treatment in patients with gastrointestinal malignancies.

\begin{tabular}{|c|c|c|c|c|c|}
\hline Authors & Cancer type & $\mathbf{n}$ & $\mathbf{T}$ & M & Results \\
\hline $\begin{array}{l}\text { West et } \\
\text { al. } 2014\end{array}$ & $\begin{array}{l}\text { Locally advanced } \\
\text { resectable rectal } \\
\text { cancer }\end{array}$ & $\begin{array}{c}25 \\
17 \overbrace{}^{\lambda}\end{array}$ & nCRT & CPET & $\begin{array}{l}\downarrow \text { Oxygen uptake at ventilatory } \\
\text { anaerobic threshold } \\
\downarrow \text { Oxygen uptake at peak exercise } \\
\text { Dose-limiting toxicity }(n=3)\end{array}$ \\
\hline $\begin{array}{l}\text { West et } \\
\text { al. } 2014\end{array}$ & $\begin{array}{l}\text { Locally advanced } \\
\text { resectable rectal } \\
\text { cancer }\end{array}$ & $\begin{array}{c}12 \\
100^{7}\end{array}$ & nCRT & CPET & $\begin{array}{l}\downarrow \text { Oxygen uptake at ventilatory } \\
\text { anaerobic threshold } \\
\downarrow \text { Oxygen uptake at peak exercise } \\
\downarrow \text { Muscle mitochondrial capacity in vivo }\end{array}$ \\
\hline $\begin{array}{l}\text { West et } \\
\text { al. } 2015\end{array}$ & $\begin{array}{l}\text { Locally advanced } \\
\text { resectable rectal } \\
\text { cancer }\end{array}$ & $\begin{array}{c}35 \\
23{ }^{\lambda}\end{array}$ & nCRT & CPET & $\begin{array}{l}\downarrow \text { Oxygen uptake at ventilatory } \\
\text { anaerobic threshold }\end{array}$ \\
\hline $\begin{array}{l}\text { Dalal et } \\
\text { al. } 2012\end{array}$ & $\begin{array}{l}\text { Inoperable locally } \\
\text { advanced } \\
\text { pancreatic cancer }\end{array}$ & $\begin{array}{c}41 \\
18{ }^{\lambda}\end{array}$ & nCRT & CT L3 & $\begin{array}{l}\downarrow \text { Skeletal muscle mass } \\
\downarrow \text { body mass ( } n=33 \text { ), median loss } 4.7 \% \text {. } \\
+ \text { correlation loss muscle mass and loss } \\
\text { body mass }\end{array}$ \\
\hline $\begin{array}{l}\text { Daly et } \\
\text { al. } 2018\end{array}$ & Foregut cancer & $\begin{array}{c}225 \\
1500^{-1}\end{array}$ & $\mathrm{nCT}$ & CT L3 & $\begin{array}{l}\downarrow \text { Skeletal muscle mass } \\
\uparrow \text { Prevalence of sarcopenia ( } 40.5 \% \text { to } \\
49.1 \% \text { ) }\end{array}$ \\
\hline $\begin{array}{l}\text { Palmela } \\
\text { et al. } \\
2017\end{array}$ & $\begin{array}{l}\text { Locally advanced } \\
\text { gastric cancer }\end{array}$ & $\begin{array}{c}48 \\
33{ }^{1}\end{array}$ & nCRT & CT L3 & $\begin{array}{l}\downarrow \text { Skeletal muscle mass } \\
\text { Association sarcopenia and early } \\
\text { termination nCT } \\
\text { Dose-limiting toxicity }(n=22) \\
\text { Early treatment termination }(n=17)\end{array}$ \\
\hline $\begin{array}{l}\text { Heus et } \\
\text { al. } 2016\end{array}$ & $\begin{array}{l}\text { Locally advanced } \\
\text { resectable rectal } \\
\text { cancer }\end{array}$ & $\begin{array}{c}74 \\
39{ }^{\lambda}\end{array}$ & nCRT & CT L3 & $\uparrow$ Skeletal muscle mass \\
\hline
\end{tabular}

Abbreviations: $\mathrm{CPET}=$ cardiopulmonary exercise testing; $\mathrm{CT}=$ computed tomography; $\mathrm{L} 3=$ third lumbar vertebrae; $\mathrm{M}=$ measurement method; $\mathrm{nCRT}=$ neoadjuvant chemoradiotherapy; $\mathrm{nCT}=$ neoadjuvant chemotherapy; $\mathrm{T}=$ treatment.

$\hat{\jmath}=$ male participants; $\downarrow=$ decrease; $+=$ positive

These findings demonstrate the negative side effects of chemoradiotherapy and emphasize the importance of sufficient aerobic capacity and skeletal muscle mass for the continuation and completion of the neoadjuvant treatment. These parameters are important for the consideration of preoperative intervention options, like prehabilitation, as well as for postoperative outcomes and survival in patients with gastrointestinal malignancies. To the best of our knowledge, there are currently no studies describing the effects of nCRT on physical fitness and skeletal muscle measurements in patients with rectal cancer. The aim of this study was therefore to evaluate changes in performance-based physical fitness and computed tomography (CT) derived skeletal muscle measurements, before and after nCRT in single subjects with locally advanced rectal cancer scheduled for elective abdominal resection. 


\section{METHODS}

\section{Participants}

Data evaluated in this observational longitudinal study consisted of routine care data of patients with locally advanced rectal cancer scheduled for nCRT at the Maastricht University Medical Center (Maastricht UMC+). Patients were referred to the physical therapy department for a screening of physical fitness before and after nCRT as part of the usual preoperative follow-up. From January 2016 until June 2018, all consecutive patients with locally advanced rectal cancer $>18$ years of age who underwent nCRT were included. This study was performed in accordance with International Conference on Harmonization-Good Clinical Practice guidelines and the medical ethical committee of the Maastricht UMC+ decided (15-4-234) that this study met the ethical policies of the Maastricht UMC+ and the regulations of the Dutch government. Patients gave written informed consent for the use of routine care data for research purposes. This manuscript is reported according to the STROBE guidelines for observational studies.

\section{Neoadjuvant chemoradiotherapy}

All consecutive patients received standardized nCRT during a period of 5.5 weeks. Radiotherapy consisted of 45 Gy in 28 fractions of 1.8 Gy. Capecitabine (825 - 1000 $\mathrm{mg} / \mathrm{m}^{2} \mathrm{bid}$ ), an oral fluoropyrimidine chemotherapeutic agent was administered on the same days that radiotherapy was performed [2]. Toxicity and side effects of nCRT were collected from the electronic patient files, documented according to the common terminology criteria for adverse events (CTCAE). Additionally, the world health organization (WHO) performance score was collected from patient files. This score is used to quantify the general well-being from patients and ranges from 0 (functioning without restrictions) to 5 (deceased).

\section{Performance-based physical fitness}

As part of usual care, patients visited the hospital physical therapist for a preoperative screening of physical fitness before the start of nCRT (T0) and after completing nCRT (T1). Following nCRT, patients were scheduled for surgery: however, in case of a clinical complete response after nCRT, patients were admitted to a "wait and see policy" which consists of omission of surgery, with close clinical and radiological follow-up [23].

\section{Aerobic capacity}

The steep ramp test (SRT) is a short and practical maximal exercise test [24], of which its primary outcome measure (the achieved maximal work rate, in Watt) has been reported to be strongly correlated to objectively measured aerobic capacity (peak oxygen uptake) during maximal cardiopulmonary exercise testing in different populations [25], among them adult cancer survivors $[26,27]$. An adjusted protocol of the original SRT was used, in 
which patients started with two minutes of unloaded cycling (warm-up phase), followed by a rapidly increase in work rate of 10 watts every 10 seconds until voluntary exhaustion despite strong verbal encouragement. Patients were instructed and verbally coached to maintain at a pedaling frequency of 70-80 rotations per minute throughout the test. When a patient was not able to maintain the pedaling speed $\geq 60 \mathrm{rpm}$, the test was ended. The maximal work rate $\left(\mathrm{WR}_{\text {peak }}\right)$ at which the pedaling frequency definitely dropped $<60 \mathrm{rpm}$ was the primary outcome measure. Heart rate and peripherally measured oxygen saturation were measured continuously throughout the test. Before and directly after finishing the test, ratings of perceived exertion (Borg score 1-10) were measured.

\section{Functional mobility}

Functional mobility was measured with the timed up-and-go (TUG) test and the twominute walk test (2MWT). With the TUG test, the time needed to rise from a chair, walk three meters, turn, walk back to the chair, and sit down again, was measured in seconds [28]. In the 2MWT the patient was instructed to walk as far as possible in two minutes (running was not allowed) on an equal surface of 15 meters (hallway). Patients were allowed to take one or more rests during the test. The use of a walking aid or orthoses was accepted. Patients were instructed to stop walking at two minutes and the walking distance was administered in meters.

\section{Muscle strength}

Handgrip strength (in $\mathrm{kg}$ ) as measured with a hand held dynamometer (JAMAR $®$ Hydraulic Hand Dynamometer, JAMAR, Patterson Medical Holdings, Inc., Illinois, USA) was performed to provide an indication of the patient's overall muscle strength. Patients were seated in a chair with their elbow flexed at $90^{\circ}$ and the forearm in a neutral position without any arm support from the chair. Patients performed the test three times with the dominant hand, of which the highest value $(\mathrm{kg})$ was reported [29].

\section{Self-reported functional capacity in performing activities of daily living}

The patient's perception of functional capacity in performing activities of daily living was measured using the Duke activity status index (DASI). The DASI is a questionnaire with twelve items, corresponding with common activities of daily living linked to a particular metabolic equivalent of task (MET) score [30].

\section{CT-derived skeletal muscle measurements}

A contrast-enhanced CT scan of the abdomen was performed before the start of nCRT and after completion of nCRT as part of routine preoperative cancer staging. On these images, the cross-sectional skeletal muscle area (SMA, in $\mathrm{cm}^{2}$ ) at the level of the third lumbar vertebra (L3) was determined. SMA at this level is highly correlated with total body skeletal muscle mass (Pearson correlation, $r=0.71-0.92$ ) [31,32]. SMA in $\mathrm{cm}^{2}$ was 
normalized for body height which results in the skeletal muscle index (SMI, in $\mathrm{cm}^{2} / \mathrm{m}^{2}$ ), a measure for relative muscle mass [33]. Furthermore, SMI can be used to classify sarcopenia, for which several cancer-specific cut off values have been published $[21,34,35]$.

For analysis, a single axial slide at the level of L3 was selected and the total skeletal muscle was demarcated by using predefined validated boundaries based on the number of Hounsfield units (HU), with the following thresholds: -29 to $+150 \mathrm{HU}$. Selection and demarcation was performed by one trained and at the moment of assessment independent assessor (I.M-R) who was unaware of the study objectives and blinded for all other patient data. Following demarcation, SMA was automatically quantified in $\mathrm{cm}^{2}$ using a software program (SliceOmatic, TomoVision, Montreal, QC, Canada). The change in SMI between the CT scans was calculated as a percentage per 100 days (percentage of change divided by the number of days between the CT scans, multiplied by 100) [36]. Taking into account a measurement error of $2 \%$ based on previous literature about the accuracy of CT analysis [33], a change in SMI of less than $2 \%(-2 \%$ to $+2 \%)$ was considered as maintenance of muscle. Loss of SMI larger than $2 \%$ was defined as clinically relevant. Finally, changes in SMI were dichotomized into loss of skeletal muscle tissue ( $>2 \%$ decrease) and maintenance/gain of skeletal muscle tissue (an increase or $\leq 2 \%$ decrease), according to recommendations of Rutten et al. [33].

\section{Statistical analysis}

The Statistical Package for the Social Sciences for Windows (version 23.0; IBM, SPSS Inc., Chicago, IL, USA) was used for statistical analysis. Shapiro-Wilk tests for normality were performed in order to evaluate the data distribution of all outcome measures. Data was presented as mean and standard deviation (SD) and as median and interquartile range (IQR). As appropriate, an independent samples t-test or its nonparametric equivalent, the Mann-Whitney $\mathrm{U}$ test, was performed on outcome measures to test for significant differences between T0 (pre nCRT) and T1 (post nCRT). For comparing several subgroups in this cohort, the Mann-Whitney $\mathrm{U}$ test was performed and correlations were studied using the Pearson or Spearman correlation coefficient, as appropriate. A P-value $<0.05$ was considered statistically significant. Where applicable, Bonferroni corrections were made to overcome the problem of multiple comparisons.

\section{RESULTS}

During the study period, 205 patients with colorectal cancer were diagnosed at the Maastricht UMC+, of which 25 patients (12.2\%) were diagnosed with advanced rectal cancer and scheduled for nCRT. These patients were all screened by the hospital physical therapist prior to nCRT (T0) and after completing nCRT (T1). The median and IQR time 
between the physical therapy screening at T0 and first nCRT session of 13.0 and 7.5 days, whereas the median and IQR time between the last nCRT session and the physical therapy screening at T1 was 9.0 and 16.0 days. Figure 1 shows the flowchart of the study.

Of the total population ( $n=25)$, sixteen patients (64.0\%) underwent surgery after nCRT. Nine patients (36.0\%) did not undergo surgery as a result of a clinical complete response after nCRT (wait and see policy, omission of surgery with follow up). However, all subjects were included for analysis concerning the preoperative data, including nineteen men $(76.0 \%)$ and six women (24.0\%) with a median and IQR age of 66.0 and 10.0 years. The median and IQR time between T0 and T1 was 107.0 and 15.5 days.

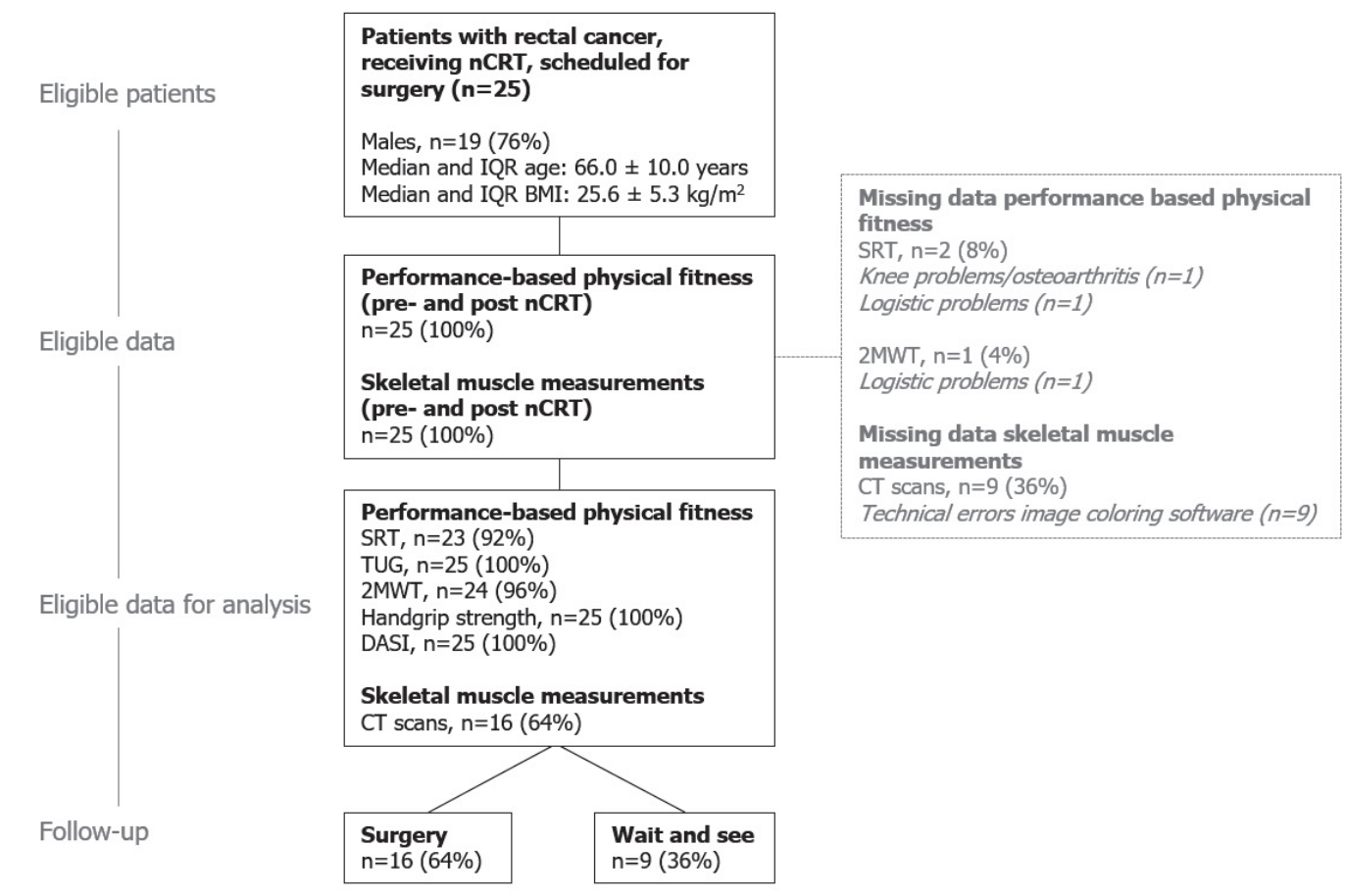

Figure 1. Flowchart of the study.

Abbreviations: $\mathrm{CT}=$ computed tomography; DASI=Duke activity status index; IQR=interquartile range; nCRT=neoadjuvant chemoradiotherapy; SRT=steep ramp test; TUG=timed-up-and-go; 2MWT=two minute walk test.

\section{Neoadjuvant chemoradiotherapy toxicity}

Table 2 shows the patient and treatment characteristics of the total population. Thirteen patients $(52.0 \%)$ experienced no toxicity of the neoadjuvant chemotherapy (CTCAE grade 0 ). Ten patients ( $40.0 \%$ ) experienced mild toxicity (CTCAE grade 1 ), including loss of body mass, fatigue, and diarrhea, and two patients (8.0\%) experienced moderate toxicity with hand-foot syndrome grade 2 (CTCAE grade 2). Eight patients $(32.0 \%)$ experienced loss of body mass during the period of nCRT. Furthermore, in three patients $(12.0 \%)$ dose-limiting toxicity or early termination of treatment during nCRT occurred. 
Table 2. Patient and treatment characteristics of the total population $(n=25)$.

\begin{tabular}{|c|c|c|c|}
\hline Variable & $n$ & $\%$ & Median (IQR) \\
\hline Sex (male) & 19 & 76.0 & \\
\hline Age (years) & & & $66.0(10.0)$ \\
\hline Body mass (kg) & & & $79.0(22.5)$ \\
\hline Body height (m) & & & $1.76(0.15)$ \\
\hline BMI $\left(\mathrm{kg} / \mathrm{m}^{2}\right)$ & & & $25.6(5.3)$ \\
\hline Comorbities (yes) & 16 & 64.0 & \\
\hline \multicolumn{4}{|l|}{ Clinical TNM stage } \\
\hline $\mathrm{T} \quad \mathrm{cT} 1$ & 0 & 0.0 & \\
\hline cT2 & 1 & 4.0 & \\
\hline cT3 & 16 & 64.0 & \\
\hline cT4 & 8 & 32.0 & \\
\hline cTx & 0 & 0.0 & \\
\hline $\mathrm{N} \quad \mathrm{cN} O$ & 2 & 8.0 & \\
\hline $\mathrm{cN} 1$ & 9 & 36.0 & \\
\hline cN2 & 14 & 56.0 & \\
\hline $\mathrm{cNx}$ & 0 & 0.0 & \\
\hline M $\quad \mathrm{CMO}$ & 22 & 88.0 & \\
\hline $\mathrm{CM} 1$ & 1 & 4.0 & \\
\hline $\mathrm{cMx}$ & 2 & 8.0 & \\
\hline \multicolumn{4}{|l|}{ Prediagnostic loss of body mass $(\mathrm{kg})$} \\
\hline Yes & 9 & 36.0 & $5.0(3.5)$ \\
\hline No & 16 & 64.0 & \\
\hline \multicolumn{4}{|l|}{ Loss of body mass during nCRT $(\mathrm{kg})$} \\
\hline yes & 8 & 32.0 & $3.0(2.8)$ \\
\hline No & 17 & 68.0 & \\
\hline \multicolumn{4}{|l|}{ WHO performance status score } \\
\hline 0 & 17 & 68.0 & \\
\hline 1 & 8 & 32.0 & \\
\hline \multicolumn{4}{|l|}{ CTCAE grade } \\
\hline 0 & 13 & 52.0 & \\
\hline 1 & 10 & 40.0 & \\
\hline 2 & 2 & 8.0 & \\
\hline \multicolumn{4}{|l|}{ Surgery after nCRT } \\
\hline Yes & 16 & 64.0 & \\
\hline Laparoscopic procedure & 14 & 87.5 & \\
\hline Open procedure & 2 & 12.5 & \\
\hline No (wait and see policy) & 9 & 36.0 & \\
\hline
\end{tabular}

Abbreviations: $\mathrm{BMI}=$ body mass index; $\mathrm{CTCAE}=$ common terminology criteria for adverse events; $\mathrm{IQR}=$ interquartile range; $\mathrm{nCRT}=$ neoadjuvant chemoradiotherapy; TNM=tumor, node, metastasis; WHO $=$ World Health Organization. 


\section{Chapter 4}

\section{Performance-based physical fitness}

The SRT could not be performed by two patients $(8.0 \%)$, whereas the 2MWT was not performed by one patient (4.0\%) (see Figure 1 ). Table $3 a$, and Figure $2 a$ and $2 b$ show the detailed data on performance-based physical fitness. Large inter-individual variation was observed between T0 and T1 concerning performance-based physical fitness. There was a statistically significant decrease in aerobic capacity after completing nCRT $(P=0.033)$. Although not statistically significant, data on muscle strength and functional mobility demonstrates a trend towards worse scores following nCRT. Regarding self-reported functional capacity in performing activities of daily living, six patients $(24.0 \%)$ reported a lower DASI score at T1, one patient (4.0\%) reported a higher DASI score at $\mathrm{T} 1$, and eighteen patients $(72.0 \%)$ reported a DASI score at T1 that was equivalent to T0.

\section{Computed tomography-derived skeletal muscle measurements}

CT images at the two time points of all patients were available: however, due to technical issues pre and/or post nCRT CT images could not be evaluated for SMA in nine patients (36.0\%). The overall scores on performance-based physical fitness of these nine patients did not differ from the total population. Consequently, pre- and post-nCRT data on SMA of sixteen patients $(64 \%)$ were complete and therefore available for analysis. Table $3 \mathrm{~b}$, and Figure $2 \mathrm{~b}$ show detailed data on body mass and SMI. A statistically significant decrease in SMI $(>2 \%)$ was found after nCRT when compared to baseline values $(P=0.005)$. In eight patients $(50 \%)$, the change in SMI was less than $2 \%(-2.0 \%$ to $+2.0 \%)$, which is defined as a maintenance of muscle mass. An additional subgroup analysis showed no significant differences in changes in performance-based physical fitness over time (T1 versus T0) between patients with a SMI decrease $>2.0 \%(n=8)$ and patients with a SMI decrease $\leq 2.0 \%(n=8)$. Correlations between the changes in SMI and the changes in performance-based physical fitness ranged between: -0.338 and 0.266 (Spearman's rho). Figure 3 shows CT scans of two individual patients before and after nCRT. 


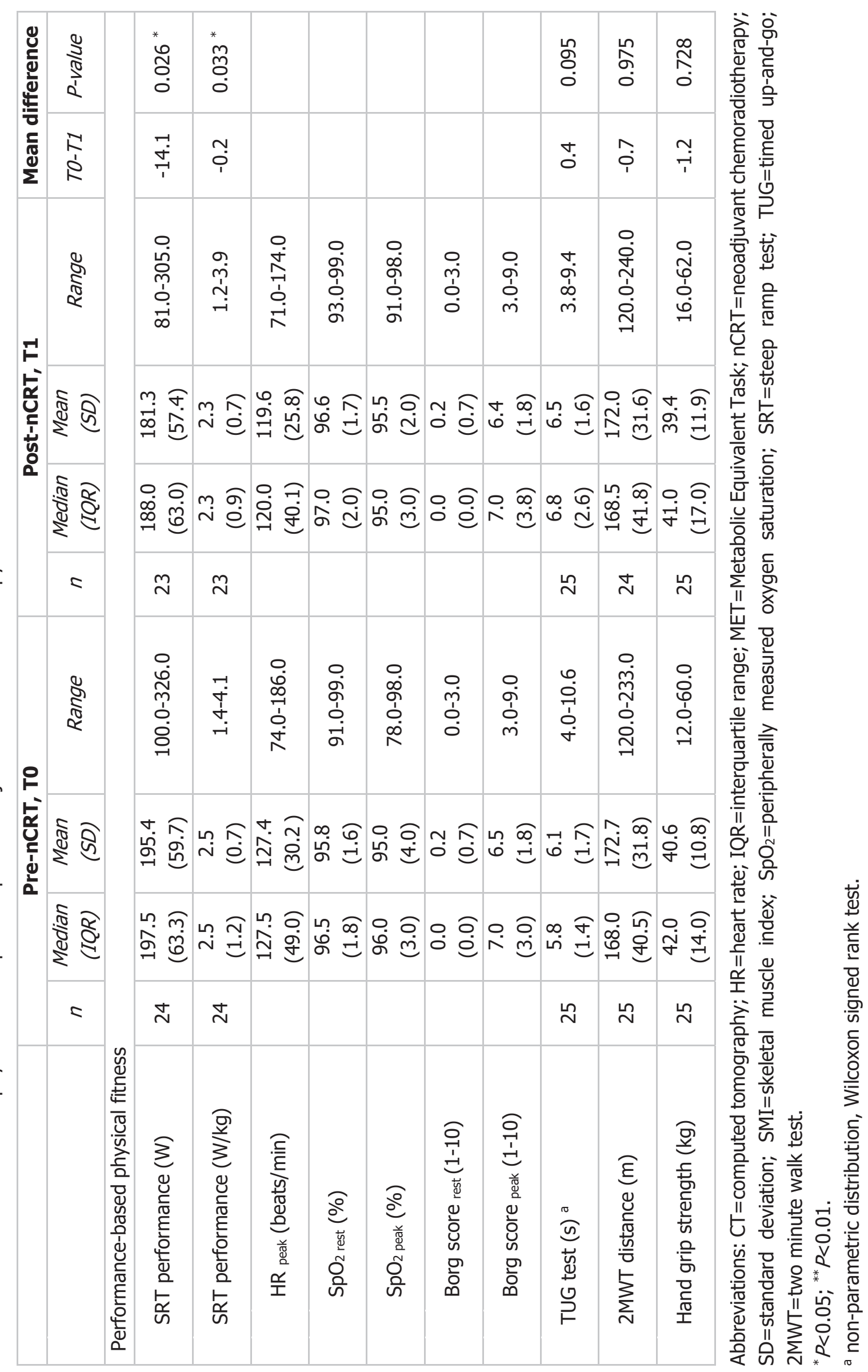




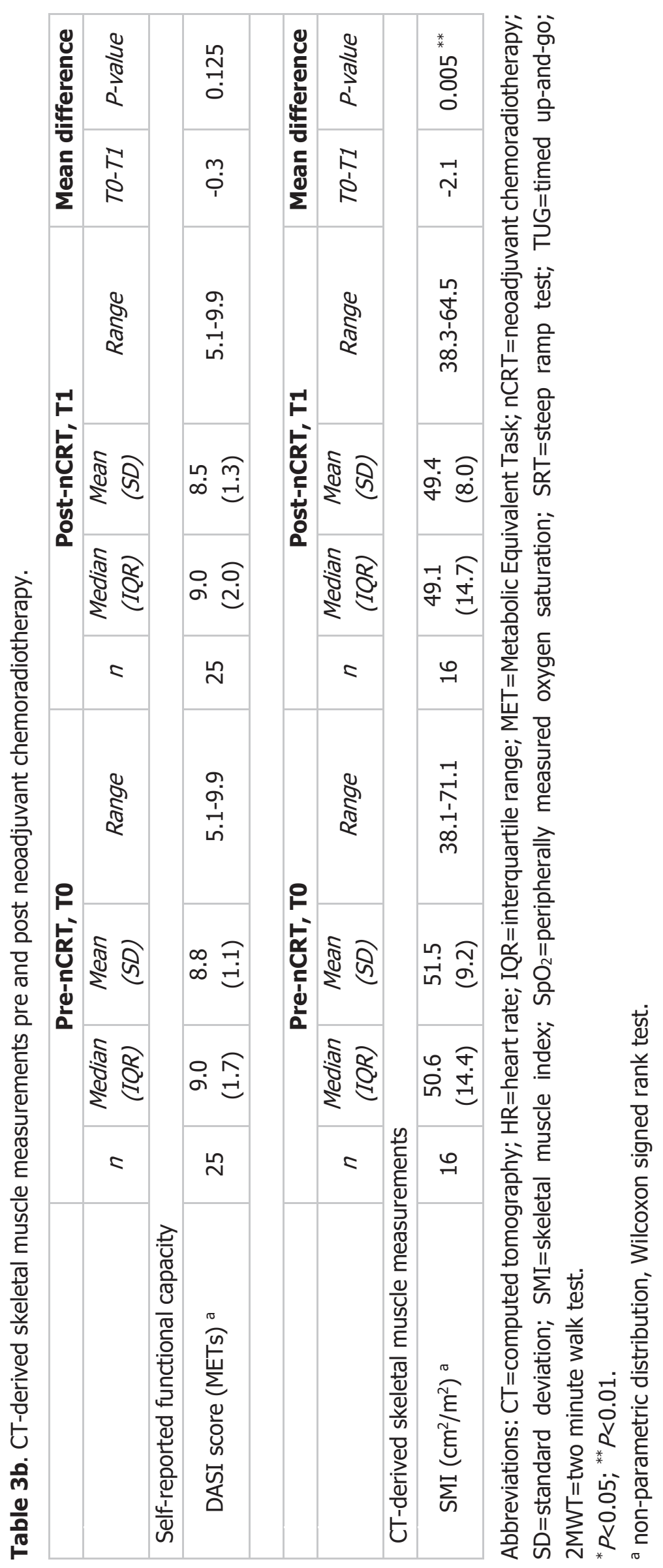



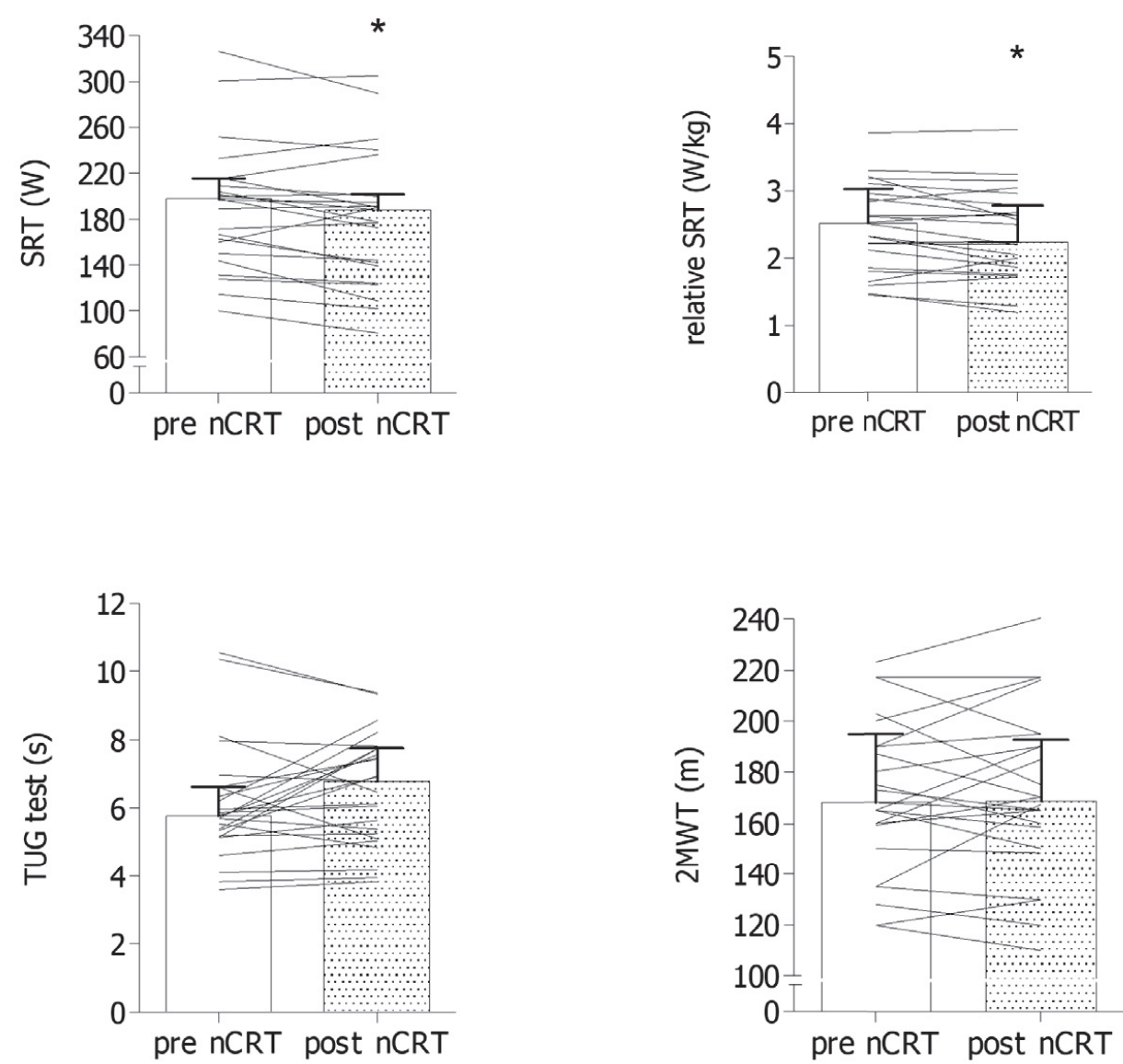

Figure 2a. Pre- and post-nCRT values of physical fitness in 25 single subjects.

White bars: Performance-based physical fitness. Abbreviations: nCRT=neoadjuvant chemoradiotherapy; SRT=steep ramp test; TUG=timed-up-and-go; $2 \mathrm{MWT}=$ two minute walk test; * Statistically significant difference. 

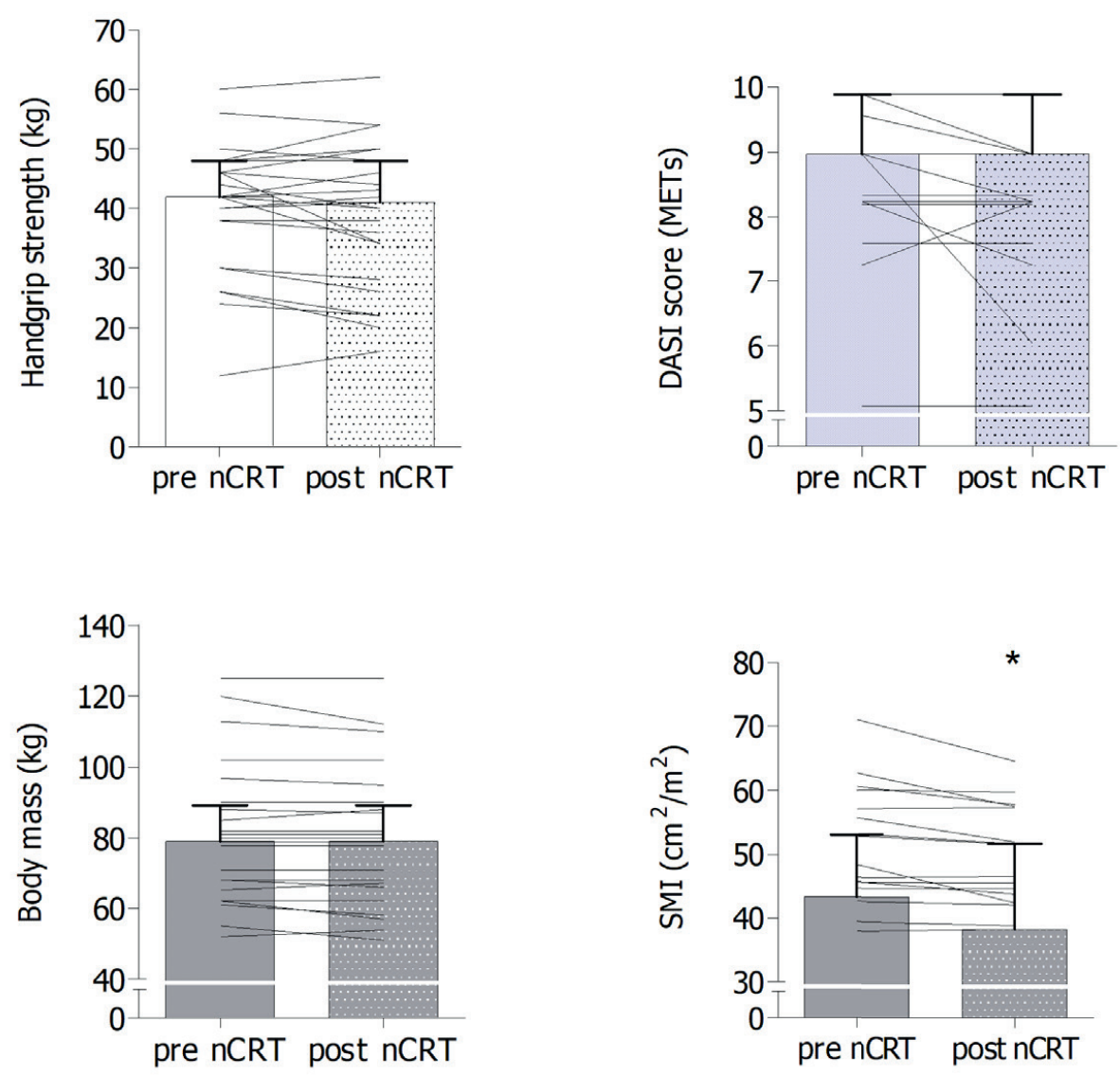

Figure 2b. Pre- and post-nCRT values in 25 single subjects with data on physical fitness $(n=25)$, body mass $(n=25)$, and SMI $(n=16)$.

White bars: Performance-based physical fitness. Light grey bars: Self-reported functional capacity in performing activities of daily living Dark grey bars: Body mass and CT-derived skeletal muscle measurements. Abbreviations: DASI=Duke activity status index; METs=metabolic equivalent tasks; $\mathrm{nCRT}=$ neoadjuvant chemoradiotherapy; SMI=skeletal muscle index; * Statistically significant difference. 


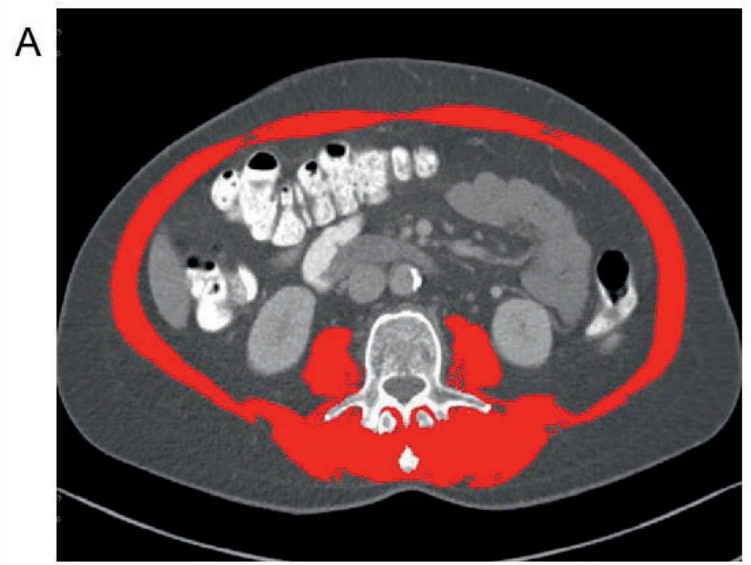

Pre-nCRT

B

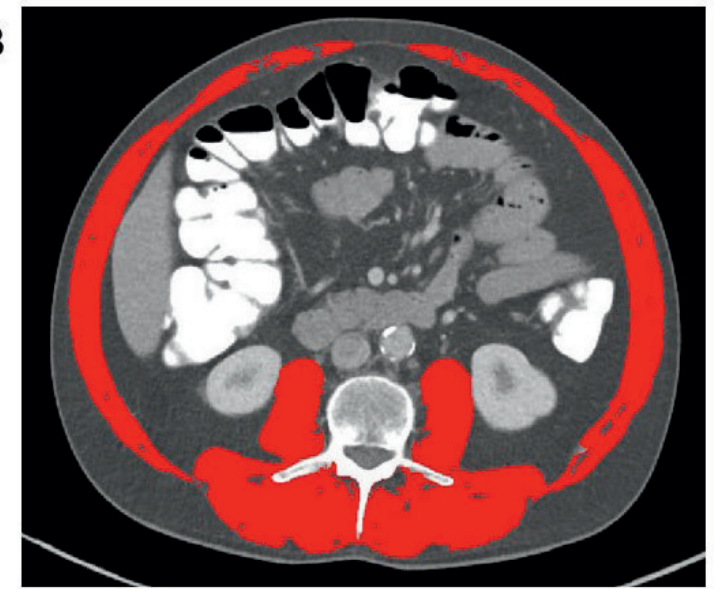

Pre-nCRT

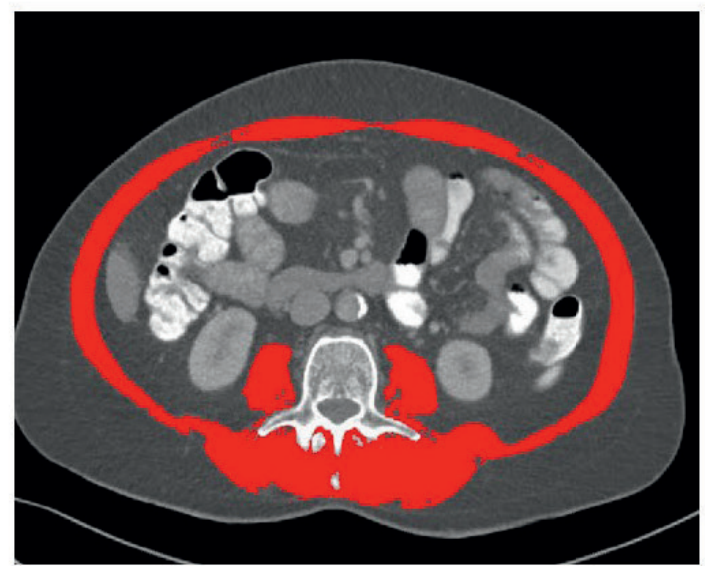

Post-nCRT

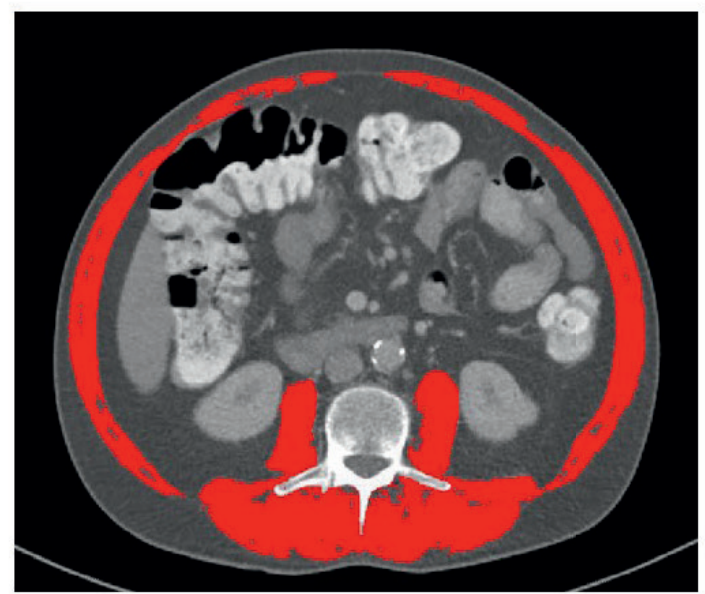

Post-nCRT

Figure 3. CT assessment of skeletal muscle with SliceOmatic software of two individual patients. Upper graphs (A): 62-year-old male with no change in SMI (+0.45\%) following nCRT; lower graphs (B): a 56year-old male, with a significant decline in SMI $(-11.37 \%)$ following nCRT. Abbreviations: CT=computed tomography; nCRT=neoadjuvant chemoradiotherapy; SMI=skeletal muscle index. 


\section{DISCUSSION}

The aim of this study was to evaluate the differences in performance-based physical fitness and CT-derived skeletal muscle measurements, before and after nCRT in 25 single subjects with locally advanced rectal cancer scheduled for elective resection. Large interindividual variation was observed concerning changes in performance-based physical fitness, skeletal muscle mass and the experience of negative side effects during nCRT. Aerobic capacity and skeletal muscle mass decreased significantly following nCRT. Only weak, non-significant correlations were found between the change in SMI and changes in performance-based physical fitness. In line with the current study, previous studies already found that aerobic capacity was decreased after nCRT as measured by cardiopulmonary exercise testing $[3,5]$. These studies showed that chemotherapy itself can affect cardiorespiratory function, microcirculatory function [37], and physical activity [38]; however, exact physiological mechanisms remain elusive. There is currently no literature available about the change in physical fitness following nCRT as objectified by other practical performance-based tests (e.g., incremental shuttle walk test, timed-upand-go test). This makes it difficult to compare our data to other studies. The decrease in skeletal muscle mass can be explained by cancer-induced cachexia $[38,39]$ and there is a possible role for oxidative stress damaging, due to the chemotherapy treatment [40]. The decrease in skeletal muscle is comparable with literature on patients with other gastrointestinal malignancies receiving neoadjuvant chemo(radio)therapy [19-21]. Previous literature about the relation between skeletal muscle mass and disease prognosis showed that the negative change in skeletal muscle mass seems of more importance than muscle mass at one single time point. Liu et al. showed that low psoas muscle index at baseline was not associated with a poor prognosis in patients with esophageal cancer whereas a decrease in psoas muscle index had a high correlation with a poor prognosis [41]. Rutten et al. showed that patients with ovarian cancer with a decrease in SMI during neoadjuvant chemotherapy had a worse survival, whereas low SMI at a specific time point was not prognostic for overall survival [36]. Next to SMI, sarcopenia is associated with survival, surgical complications, and treatment-related toxicities in patients with colorectal cancer $[42,43]$. A post-hoc analysis in our cohort showed that the incidence of sarcopenia, according to the criteria from martin et al. [34], was higher after completing nCRT than at baseline. These findings emphasize the importance of monitoring (physical) status to guide the patients through their medical treatments and intervene when necessary, for example with nutrition, medication, or

physical therapy. Hence, for patients that develop sarcopenia during nCRT an intervention during this treatment course can be extra useful.

Further analysis of the individual data of this sample showed that patients with a decline in physical fitness after nCRT seem to experience negative side effects during nCRT more often. However, elucidating the inter-individual differences over time was difficult to 
establish, because there were only two time points in which performance-based physical fitness and skeletal muscle mass were measured. Additionally, there is no insight in confounding factors as physical activity during nCRT. Monitoring performance-based physical fitness and skeletal muscle mass with a higher frequency over time in combination with wearables for physical activity can be helpful to overcome this problem. Hence, insight in (the change of) aerobic capacity and skeletal muscle mass during nCRT can help health care professionals to guide patients in their treatment course and advise them about physical activity, nutrition, and prehabilitation, to improve patient satisfaction and other patient outcomes as quality of life, and (long-term) clinical outcomes.

When this study cohort was compared with the total cohort of patients with colorectal cancer undergoing colorectal resection at our hospital in the same time period $(n=180)$, the nCRT cohort had a better performance-based physical fitness at the baseline preoperative screening of physical fitness (T0). This could suggest some kind of (unconscious) pre-selection by the health care professional, in which patients with locally advanced rectal cancer with a better physical fitness are more often considered eligible for nCRT compared to patients with a lower physical fitness. With the current knowledge and evidence about prehabilitation and its effectiveness, it may not be necessary to withhold patients from nCRT. Previous studies have shown that physical exercise training during nCRT is feasible and safe for patients with locally advanced rectal cancer $[44,45]$. Like previous studies, it would have been of interest to evaluate the association between preoperative aerobic capacity and skeletal muscle mass with postoperative outcomes $[13-15,42]$ and overall survival $[18,43]$. These possible associations were not investigated in the current study because of the rather small sample size.

In conclusion, the current study revealed a reduction in aerobic capacity and skeletal muscle mass following nCRT in patients with locally advanced rectal cancer, with large inter-individual variation concerning alterations in performance-based physical fitness, muscle mass and the experience of negative side effects. The variability between subjects requires a personalized treatment approach including frequent monitoring of physical fitness. 


\section{REFERENCES}

1. The Netherlands Comprehensive Cancer Organisation (IKNL), Cijfers over kanker. http://www.cijfersoverkanker.nl/meest-voorkomende-soorten-52.html, 2018 (accessed December 2018).

2. The Netherlands Comprehensive Cancer Organisation (IKNL), Oncoline Richtlijnen oncologische zorg. https://www.oncoline.nl/colorectaalcarcinoom, 2018 (accessed December 2018).

3. West MA, Loughney L, Barben $\mathrm{CP}$, et al., The effects of neoadjuvant chemoradiotherapy on physical fitness and morbidity in rectal cancer surgery patients, Eur J Surg Oncol. 40 (2014) 1421-1428.

4. West MA, Loughney L, Lythgoe $D$, et al., The effect of neoadjuvant chemoradiotherapy on wholebody physical fitness and skeletal muscle mitochondrial oxidative phosphorylation in vivo in locally advanced rectal cancer patients-an observational pilot study, PLoS One. 9 (2014) 1-15.

5. West MA, Loughney L, Lythgoe $D$, et al., Effect of prehabilitation on objectively measured physical fitness after neoadjuvant treatment in preoperative rectal cancer patients: a blinded interventional pilot study, $\mathrm{Br}$ J Anaesth. 114 (2015) 244-251.

6. Bosset JF, Collette L, Calais G, et al., Chemotherapy with preoperative radiotherapy in rectal cancer, N Engl J Med. 355 (2006) 1114-1123.

7. Sauer R, Liersch T, Merkel S, et al., Preoperative versus postoperative chemoradiotherapy for locally advanced rectal cancer: results of the German CAO/ARO/AIO-94 randomized phase III trial after a median follow-up of 11 years, J Clin Oncol. 30 (2012) 1926-1933.

8. Dutch Institute for Clinical Auditing (DICA) Leiden, DICA Jaarrapportage 2017: DCRA 2017. https://dica.nl/jaarrapportage-2017/dcra, 2018 (accessed december 2018).

9. Chau I, Brown G, Cunningham D, et al., Neoadjuvant capecitabine and oxaliplatin followed by synchronous chemoradiation and total mesorectal excision in magnetic resonance imagingdefined poor-risk rectal cancer, J Clin Oncol. 24 (2006) 668-674.

10. Swellengrebel HA, Marijnen CA, Verwaal VJ, et al., Toxicity and complications of preoperative chemoradiotherapy for locally advanced rectal cancer, Br J Surg. 98 (2011) 418-426.

11. Windsor PM, Potter J, McAdam K, et al., Evaluation of a fatigue initiative: information on exercise for patients receiving cancer treatment, Clin Oncol (R Coll Radiol). 21 (2009) 473-482.

12. Jack $S$, West MA, Raw $D$, et al., The effect of neoadjuvant chemotherapy on physical fitness and survival in patients undergoing oesophagogastric cancer surgery, Eur J Surg Oncol. 40 (2014) 1313-1320.

13. Snowden CP, Prentis J, Jacques B, et al., Cardiorespiratory fitness predicts mortality and hospital length of stay after major elective surgery in older people, Ann Surg. 257 (2013) 9991004.

14. West MA, Lythgoe $D$, Barben $C P$, et al., Cardiopulmonary exercise variables are associated with postoperative morbidity after major colonic surgery: a prospective blinded observational study, Br J Anaesth. 211 (2014) 665-671.

15. West MA, Parry MG, Lythgoe $D$, et al., Cardiopulmonary exercise testing for the prediction of morbidity risk after rectal cancer surgery, Br J Surg. 101 (2014) 1166-1172.

16. Levett DZ, Grocott MP, Cardiopulmonary exercise testing for risk prediction in major abdominal surgery, Anesthesiol Clin. 33 (2015) 1-16.

17. Hennis PJ, Meale PM, Grocott MP, Cardiopulmonary exercise testing for the evaluation of perioperative risk in non-cardiopulmonary surgery, Postgrad Med J. 87 (2011) 550-557.

18. Takeda Y, Akiyoshi T, Matsueda K, et al., Skeletal muscle loss is an independent negative prognostic factor in patients with advanced lower rectal cancer treated with neoadjuvant chemoradiotherapy, PLoS One. 13 (2018) e0195406. 
19. Dalal $S$, Hui D, Bidaut $L$, et al., Relationships among body mass index, longitudinal body composition alterations, and survival in patients with locally advanced pancreatic cancer receiving chemoradiation: a pilot study, J Pain Symptom Manage. 44 (2012) 181-191.

20. Daly LE, Ní Bhuachalla ÉB, Power DG, et al., Loss of skeletal muscle during systemic chemotherapy is prognostic of poor survival in patients with foregut cancer, J Cachexia Sarcopenia Muscle. 9 (2018) 315-325.

21. Palmela C, Velho S, Agostinho L, et al., Body composition as a prognostic factor of neoadjuvant chemotherapy toxicity and outcome in patients with locally advanced gastric cancer, J. Gastric cancer. 17 (2017) 74-87.

22. Heus $\mathrm{C}$, Cakir $\mathrm{H}$, Lak $\mathrm{A}$, et al., Visceral obesity, muscle mass and outcome in rectal cancer surgery after neo-adjuvant chemo-radiation, J Surg. 29 (2016) 159-164.

23. Maas M, Beets-Tan RG, Lambregts $D M$, et al., Wait-and-see policy for clinical complete responders after chemoradiation for rectal cancer, J Clin Oncol. 29 (2011) 4633-4640.

24. Meyer K, Samek L, Schwaibold M, et al., Physical responses to different modes of interval exercise in patients with chronic heart failure--application to exercise training, Eur Heart J. 17 (1996) 1040-1047.

25. Bongers $\mathrm{BC}$, de Vries SI, Helders $\mathrm{PJ}$, et al., The steep ramp test in healthy children and adolescents: reliability and validity, Med Sci Sports Exerc. 45 (2013) 366-371.

26. De Backer IC, Schep G, Hoogeveen A, et al., Exercise testing and training in a cancer rehabilitation program: the advantage of the steep ramp test, Arch Phys Med Rehabil. 88 (2007) 610-616.

27. Stuiver MM, Kampshoff CS, Persoon S, et al., Validation and refinement of prediction models to estimate exercise capacity in cancer survivors using the steep ramp test, Arch Phys Med Rehabil. 98 (2017) 2167-2173.

28. Gautschi OP, Corniolam MV, Joswig $\mathrm{H}$, et al., The timed up and go test for lumbar degenerative disc disease, J Clin Neurosci. 22 (2015) 1943-1948.

29. Fess E, Grip strength, in: American society of hand therapists, clinical assessment recommendations, $2^{\text {nd }}$ edition, The Society, Chicago, 1992, pp. 41-45.

30. Hlatky MA, Boineau RE, Higginbotham MB, et al., A brief self-administered questionnaire to determine functional capacity (the Duke activity status index), Am J Cardiol. 64 (1989) 651654.

31. Shen W, Punyanitya $M$, Wang $Z$, et al., Total body skeletal muscle and adipose tissue volumes: estimation from a single abdominal cross-sectional image, J Appl Physiol. 97 (2004) 2333-2338.

32. Mourtzakis M, Prado CM, Lieffers JR, et al., A practical and precise approach to quantification of body composition in cancer patients using computed tomography images acquired during routine care, Appl Physiol Nutr Metab. 33 (2008) 997-1006.

33. Baumgartner RN, Koehler KM, Gallagher $D$, et al., Epidemiology of sarcopenia among the elderly in New Mexico, Am J Epidemiol. 147 (1998) 755-763.

34. Martin L, Birdsell L, Macdonald N, et al., Cancer cachexia in the age of obesity: skeletal muscle depletion is a powerful prognostic factor, independent of body mass index, J Clin Oncol. 31 (2013) 1539-1547.

35. Martin L, Hopkins J, Malietzis G, et al., Assessment of computed tomography (CT)-defined muscle and adipose tissue features in relation to short-term outcomes after elective surgery for colorectal cancer: a multicenter approach, Ann Surg Oncol. 25 (2018) 2669-2680.

36. Rutten IJ, van Dijk DP, Kruitwagen RF, et al., Loss of skeletal muscle during neoadjuvant chemotherapy is related to decreased survival in ovarian cancer patients, J Cachexia Sarcopenia Muscle. 7 (2016) 458-466. 


\section{Chapter 4}

37. Karvunidis T, Chvojka J, Lyska D, et al., Septic shock and chemotherapy-induced cytopenia: effects on microcirculation, Intensive Care Med. 38 (2012) 1336-1344.

38. Gilliam LA, St Clair DK, Clair DK, Chemotherapy-induced weakness and fatigue in skeletal suscle: the role of oxidative stress, Antioxid Redox Signal. 15 (2011) 2543-2546.

39. Tisdale MJ, Cancer anorexia and cachexia, Nutrition. 17 (2001) 438-442.

40. Powers SK, Jackson MJ, Exercise-induced oxidative stress: cellular mechanisms and impact on muscle force production, Physiol Rev. 88 (2008) 1243-1276.

41. Liu J, Motoyama S, Sato Y, et al., Decreased skeletal muscle mass after neoadjuvant therapy correlates with poor prognosis in patients with esophageal cancer, Anticancer Res. 36 (2016) 6677-6685.

42. Boer BC, de Graaff F, Brusse-Keizer M, et al., Skeletal muscle mass and quality as risk factors for postoperative outcome after open colon resection for cancer, Int J Colorectal Dis. 31 (2016) 1117-1124.

43. van Vledder MG, Levolger $\mathrm{S}$, Ayez $\mathrm{N}$, et al., Body composition and outcome in patients undergoing resection of colorectal liver metastases, Br J Surg. 99 (2012) 550-557.

44. Heldens AF, Bongers BC, de Vos-Geelen J, et al., Feasibility and preliminary effectiveness of a physical exercise training program during neoadjuvant chemoradiotherapy in individual patients with rectal cancer prior to major elective surgery, Eur J Surg Oncol. 42 (2016) 1322-1330.

45. Singh F, Galvão DA, Newton RU, et al., Feasibility and preliminary efficacy of a 10-week resistance and aerobic exercise intervention during neoadjuvant chemoradiation treatment in rectal cancer patients, Integr Cancer Ther. 17 (2018) 952-959. 




\section{Chapter}

Feasibility and preliminary effectiveness of a physical exercise training program during neoadjuvant chemoradiotherapy in individual patients with rectal cancer prior to major elective surgery

Aniek F. Heldens

Bart C. Bongers

Judith de Vos-Geelen

Nico L. van Meeteren

Antoine F. Lenssen

European Journal of Surgical Oncology 2016;42:1322-1330. 


\section{Chapter 5}

\section{ABSTRACT}

\section{Background.}

Diverse fractions of patients with locally advanced resectable rectal cancer receive neoadjuvant chemoradiotherapy (nCRT). nCRT is known to decrease physical fitness, an undesirable side effect. This pilot aimed to determine the feasibility and preliminary effectiveness of a supervised outpatient physical exercise training program during nCRT in these patients.

\section{Methods.}

We included 13 out of 20 eligible patients (11 males, mean \pm standard deviation (SD) age: $59.1 \pm 19.7$ years) with rectal cancer who participated in the exercise training program during nCRT. Feasibility was determined by adherence and number of adverse events. Physical fitness was compared at baseline (B), after five week of training (T1) and ten weeks of training (T2), and eight weeks postoperatively (T3) using repeatedmeasures analysis of variance.

\section{Results.}

Nine patients $(69.2 \%)$ completed the program without adverse events. Four patients dropped out. The program was feasible and safe, with a total attendance rate of $95.7 \%$. Leg muscle strength (mean \pm SD: $104.0 \pm 32.3$ versus $144.8 \pm 45.6 \mathrm{~kg} ; P<0.001$ ) and arm muscle strength (mean $\pm \mathrm{SD}: 48.7 \pm 13.8 \mathrm{~kg}$ versus $36.1 \pm 11.0 \mathrm{~kg}, P=0.002$ ) increased significantly between $B$ and $T 2$, respectively. A slight, non-significant, increase in functional exercise capacity was found.

\section{Conclusions.}

This pilot demonstrated that a supervised outpatient physical exercise training program for individual patients with locally advanced resectable rectal cancer during nCRT is feasible for a large part of the patients, safe and seems able to prevent an often seen decline in physical fitness during nCRT. A larger study into the cost-effectiveness of this approach is warranted. 


\section{INTRODUCTION}

Nowadays, colorectal cancer is the second most common diagnosis of cancer in the Netherlands [1]. In 2014, 15003 new cases of colorectal cancer (69.2\% colon, 30.8\% rectal) were registered [1]. In 2014, 2846 patients diagnosed with rectal cancer underwent rectal resection surgery (29\% aged $>75$ years), in which the 30-day complication rate and the 30 -day mortality rate were $37 \%$ and $1.1 \%$, respectively [2].

Patients with locally advanced rectal cancer (Tumor, Node, Metastasis (TNM) stage cT3 or cT4N with involvement of the mesorectal fascia and/or extramesorectal lymph node metastases) are considered for an extensive treatment protocol of neoadjuvant chemoradiotherapy (nCRT) [3-6] to improve long-term outcome. nCRT aims to control local disease and improve resectability by downsizing the tumor and hereby increasing negative resection margins $[6,7]$. In the Netherlands, $34 \%$ of the patients scheduled for rectal resection received nCRT in 2014 [2]. However, chemoradiotherapy is a regimen with a high toxicity profile, which can lead to extensive diarrhoea, hand-foot syndrome, cardiotoxicity and haematological toxicity [8]. Additionally, chemoradiotherapy has negative physical side effects, of which fatigue [9] and a decrease in cardiorespiratory fitness $[3-5,10]$ are the most common. Recently published studies explored the impact of neoadjuvant therapy on cardiorespiratory fitness prior to rectal resection [3-5]. Following nCRT, oxygen uptake $\left(\mathrm{VO}_{2}\right)$ at the ventilatory threshold and $\mathrm{VO}_{2}$ at peak exercise $\left(\mathrm{VO}_{2 \text { peak }}\right)$ were reduced, as objectively measured during cardiopulmonary exercise testing [3-5].

Preoperative cardiorespiratory fitness has a consistent positive relation with postoperative outcomes in major abdominal surgery [11-15]. Major abdominal surgery is associated with an increase of oxygen demand of $40 \%$ or more, which must be met by an increase in cardiac output or an increase in oxygen extraction $[16,17]$. Patients with a higher preoperative level of cardiorespiratory fitness may have a greater physiological reserve to tolerate this metabolic stress [14]. Patients who receive nCRT may have and/or gradually develop a lower physiological reserve to tolerate the metabolic stress of surgery, because of the decrease in cardiorespiratory fitness caused by nCRT [3-5] and a decrease in physical activity [3]. These findings suggest that preservation or even improvement of cardiorespiratory fitness may be important for rectal cancer patients exposed to the dual challenges of nCRT and major abdominal surgery. A poor cardiorespiratory fitness in these patients is associated with postoperative outcomes [4]. A recent study from West et al. [3] showed that a preoperative physical exercise training program following nCRT was feasible and may be beneficial for patients undergoing rectal resection surgery, as cardiorespiratory fitness returned to baseline values within six weeks after the completion of nCRT. 


\section{Chapter 5}

There is currently no literature available on physical exercise programs during nCRT in patients with rectal cancer aiming to slow-down or prevent a decline in cardiorespiratory fitness. Therefore the primary aim of this pilot study was to determine the feasibility of a supervised outpatient physical exercise training program during nCRT in patients with rectal cancer. Secondly, the preliminary effectiveness of the physical exercise training program during nCRT on physical fitness (functional exercise capacity and muscle strength), fatigue, and quality of life of individual patients was studied.

\section{METHODS}

\section{Participants}

This study was performed between April 2014 and April 2015 as a single group prospective pilot study, in which the medical oncologist referred patients receiving nCRT to the physical therapy department for participation in a physical exercise training program. Patients were included when they were $>18$ years of age, diagnosed with locally advanced resectable rectal cancer, and undergoing nCRT based on CTNM stage. Patients were excluded when their medical status contraindicated exercise or when they were not capable to cooperate with the training and/or testing procedures. After evaluation, the Medical Ethical Committee of the Maastricht University Medical Center (Maastricht UMC+) decided that this study met the ethical policies of the Maastricht UMC+ and the regulations of the Dutch government. Oral informed consent was obtained from all patients.

\section{Neoadjuvant chemoradiotherapy}

All consecutive patients received standardized nCRT during a period of 5.5 weeks. Radiotherapy consisted of $45 \mathrm{~Gy}$ in 25 fractions of $1.8 \mathrm{~Gy}$ over a period of 5 weeks. In addition, in week six, a boost of three fractions of $1.8 \mathrm{~Gy}$ was performed. Capecitabine, an oral fluoropyrimidine chemotherapy, $625 \mathrm{mg} / \mathrm{m}^{2}$ bid was given continuously during 5.5 weeks. Chemotherapy consisted of oxaliplatin $130 \mathrm{mg} / \mathrm{m}^{2}$ intravenously on day one in combination with capecitabine $1000 \mathrm{mg} / \mathrm{m}^{2}$ bid orally on day one to 14 , in a three weekly cycle. During the standard waiting period after nCRT, which is necessary to induce optimal effect of the radiotherapy, another two cycles of chemotherapy were performed when possible [18].

\section{Physical exercise training program}

Throughout their complete nCRT treatment, patients attended an individual supervised outpatient physical exercise training program (two sessions a week) designed to slowdown or prevent a decline in cardiorespiratory fitness and muscular endurance capacity. The physical exercise training program started in the first week of nCRT and the duration 
was dependent on the planning for surgery for each individual patient. The program was executed at the physical therapy department of the Maastricht UMC+ and was guided alternatingly by two trained physical therapists. Additionally, patients were encouraged to be physically active at home (e.g., walking, cycling, gardening, sports). Training sessions were individual and consisted of 45 to 60 minutes of endurance and resistance exercises, at moderate exercise intensity, as described in previous studies [19], which was responsive to the Borg rating of perceived exertion on a scale of 6-20. Overall, a patient's Borg score of 13-14 was used to achieve a moderate exercise intensity and to provide an individualized, patient-tailored program. In the first week, endurance exercises (treadmill and cycle ergometer) were performed at 50-60\% of the estimated maximal heart rate (220 - age (in years)), with a duration of 15 minutes each. When patients used a beta blocker $(n=2 ; 22.2 \%)$, the Borg scale was used (score 13 or 14 for moderate intensity). Moreover, three resistance exercises were performed (leg press, chest press, and lateral pull down, TECA innovative fitness technology, Otana, Italy) at $40 \%$ of the one-repetition maximum, with three series of 15 repetitions for each muscle group, to train muscular endurance capacity. By doing so, patients became familiar with the exercise equipment and their own abilities. As of week two of the physical exercise training program, all patients indicated their Borg score after completing each training component. To maintain an adequate (moderate-intensity) training stimulus (Borg score >13), these titration-tactics were used (as advocated by Glasziou and colleagues [20]) to individually adjust exercise intensity and training duration for every next training session when patients did score $<13$. Patients were instructed to stop exercising if any unusual symptoms were experienced (e.g., dizziness or chest pain).

\section{Measurements}

\section{Feasibility}

Feasibility of the physical exercise training program during nCRT was determined by the registration of the number and severity of adverse events, as well as by adherence to the program. These data were recorded by the physical therapist, including the reasons for missing a training session. Moreover, patient motivation and satisfaction were measured after the physical exercise training program by asking patients to rate their appreciation of the content of the program, as well as the guidance during the program and the sport facilities with help of a scale from 0 to 10 . All measurements were performed by the two physical therapists that guided the program (not blinded). 


\section{Chapter 5}

\section{Preliminary effectiveness}

Changes in physical functioning parameters during the physical exercise program and nCRT were evaluated per patient by visually and statistically comparing physical fitness (functional exercise capacity and muscle strength) and perceptions of fatigue and quality of life at baseline (B), after five weeks of training (T1), after ten weeks of training (T2) and eight weeks postoperatively (T3).

\section{Functional exercise capacity}

To measure functional exercise capacity of the patients, the six-minute walk test (6MWT) was used, with maximum walking distance as primary outcome measure. The 6MWT was performed according to the American Thoracic Society guidelines [21]; however, the test was performed on a 44 meter square surface instead of a 30-meter course.

\section{Muscle strength}

Muscle strength was measured with the submaximal multiple-repetition (X-RM) test procedure for two resistance exercises. The leg extension machine (Horizontal Leg Press, TECA innovative fitness technology, Otana, Italy) and the chest press machine (Chest Press, TECA innovative fitness technology, Otana, Italy) were used to estimate maximal muscle strength of the legs and arms, respectively. For practical purposes, the lateral pull down was not used as an evaluative measurement. The patient was asked to perform as many repetitions as possible with a weight chosen by the physical therapist. Based on the number of completed repetitions, the maximal muscle strength of the patient was determined, using the Oddvar Holten diagram [22].

\section{Perception of fatigue and quality of life}

To determine the patient's perception of fatigue, patients filled out the multidimensional fatigue index (MFI) [23]. The MFI is a self-reporting questionnaire with 20 propositions about the different dimensions and consequences of fatigue. A higher score means a higher level of perceived fatigue. The short form 36 health survey (SF-36) was used to gain insight in the patient's perceived health-related quality of life. The SF-36 contains 36 questions concerning the patient's own health status perceptions in the following domains: physical, mental, and social health [24]. A higher score (percentage) corresponds with a better perceived health status.

\section{Statistical analysis}

The Statistical Package for the Social Sciences for Windows (version 20.0; IBM, SPSS Inc., Chicago, IL, USA) was used for the statistical analysis. Data were presented for each individual patient for visual analysis and as mean values \pm standard deviation (SD) for statistical analysis. Shapiro-Wilk tests for normality were performed in order to evaluate the data distribution of each measurement. Because all data were distributed 
normally, differences in test scores were examined using repeated-measures analysis of variance (ANOVA). Additional post-hoc analyses with manual Bonferroni adjustment for multiple testing were performed on the outcomes of the repeated-measures ANOVA tests to locate the exact significant differences. A P-value $<0.05$ was considered statistically significant.

\section{RESULTS}

\section{Feasibility}

Figure 1 depicts the patient flow in this study, including reasons for not participating and dropouts. Twenty patients were eligible for participating in the training program, of which five patients $(25.0 \%)$ refused participation. After baseline examination, two patients were excluded. Hence, 13 of 20 participants (65.0\%) started the physical exercise training program in the first week of their nCRT. Of these 13 patients, four $(30.8 \%)$ dropped out during the program. Eventually, nine of 13 patients $(69.2 \%)$ completed the program without any adverse events. Six patients $(66.7 \%)$ underwent elective surgery at the end of the physical exercise training program. Three patients (33.3\%), did not undergo surgery as a result of a clinical complete response after nCRT (wait and see policy, omission of surgery with follow up) [25]. Of the six patients who underwent surgery, one patient (16.7\%) refused participation in the follow up after surgery. Consequently, there are postoperative data available for five of six patients $(83.3 \%)$. Patient characteristics of the total population $(n=9)$ and the population that underwent surgery $(n=6)$ are summarized in Table 1. 


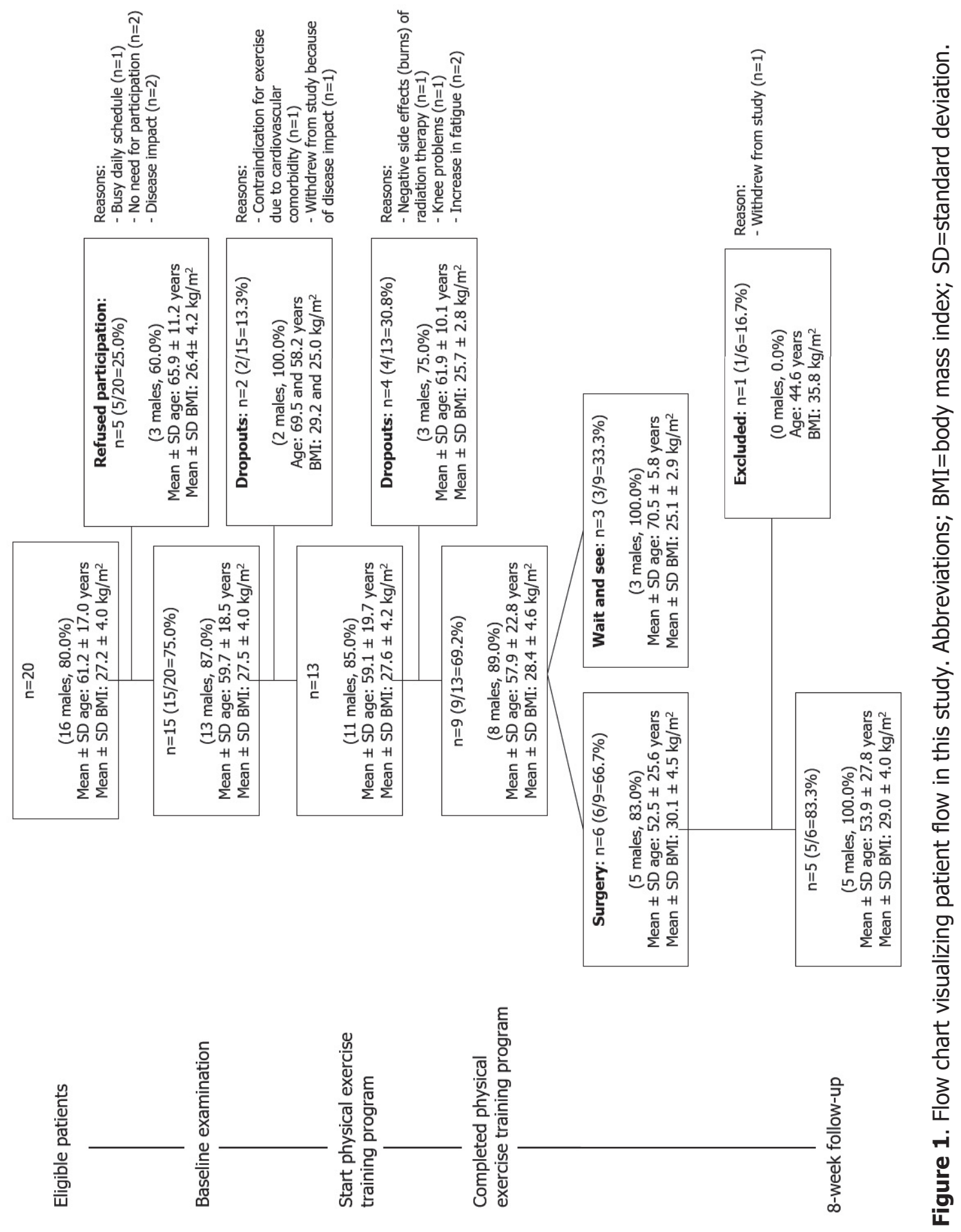


Table 1. Patient demographics and clinical characteristics.

\begin{tabular}{|c|c|c|c|c|}
\hline & \multicolumn{2}{|c|}{ Total $(n=9)$} & \multicolumn{2}{|c|}{ Surgery $(n=6)$} \\
\hline & \multicolumn{2}{|c|}{ Mean (SD) } & \multicolumn{2}{|c|}{ Mean (SD) } \\
\hline Age (years) & \multicolumn{2}{|c|}{$64.4(10.9)$} & \multicolumn{2}{|c|}{$61.3(11.9)$} \\
\hline BMI $\left(\mathrm{kg} / \mathrm{m}^{2}\right)$ & \multicolumn{2}{|c|}{$28.4(4.6)$} & \multicolumn{2}{|c|}{$30.1(4.5)$} \\
\hline & $n$ & $\%$ & $n$ & $\%$ \\
\hline Sex (males) & 8 & 89 & 5 & 83 \\
\hline Smoking (yes) & 2 & 22 & 2 & 33 \\
\hline Alcohol (yes) & 4 & 44 & 4 & 67 \\
\hline \multicolumn{5}{|l|}{ Comorbidities } \\
\hline Heart disease & 4 & 44 & 2 & 33 \\
\hline Diabetes & 1 & 11 & 1 & 17 \\
\hline COPD & 1 & 11 & 1 & 17 \\
\hline Orthopaedic & 1 & 11 & 1 & 17 \\
\hline None & 3 & 33 & 2 & 33 \\
\hline \multicolumn{5}{|c|}{ Physical activity (MET values) } \\
\hline MET 7 & 1 & 11 & 0 & 0 \\
\hline MET 8 & 1 & 11 & 1 & 17 \\
\hline MET 9 & 2 & 22 & 2 & 33 \\
\hline MET 10 & 3 & 33 & 2 & 33 \\
\hline MET 11 & 2 & 22 & 1 & 17 \\
\hline \multicolumn{5}{|c|}{ Tumor distance from anal verge $(\mathrm{cm})$} \\
\hline$<5.0$ & 5 & 56 & 3 & 50 \\
\hline $5.1-10.0$ & 3 & 33 & 2 & 33 \\
\hline$>10.1$ & 1 & 11 & 1 & 17 \\
\hline \multicolumn{5}{|l|}{ TNM stage } \\
\hline cT3 & 7 & 78 & 4 & 67 \\
\hline cT4 & 2 & 22 & 2 & 33 \\
\hline cNO & 3 & 33 & 1 & 17 \\
\hline cN1 & 1 & 11 & 1 & 17 \\
\hline cN2 & 5 & 56 & 4 & 67 \\
\hline $\mathrm{cMO}$ & 9 & 100 & 6 & 100 \\
\hline \multicolumn{5}{|l|}{ ASA score } \\
\hline I & 5 & 56 & 3 & 50 \\
\hline II & 3 & 33 & 2 & 33 \\
\hline III & 1 & 11 & 1 & 17 \\
\hline \multicolumn{5}{|l|}{ Surgical method } \\
\hline LAP LAR & & & 4 & 67 \\
\hline OPEN LAR & & & 2 & 33 \\
\hline
\end{tabular}

Abbreviations: $A S A=A m e r i c a n$ Society of Anaesthesiologists score; BMI=Body mass index $\left(\mathrm{kg} / \mathrm{m}^{2)}\right.$; $\mathrm{COPD}=$ Chronic obstructive pulmonary disease; $I Q R=$ Interquartile range; $L A P=L a p a r o s c o p i c ; L A R=L o w$ anterior resection=; MET=Metabolic Equivalent (1 MET equals $3.5 \mathrm{ml} / \mathrm{kg} / \mathrm{min}$ ); SD=Standard deviation; TNM=Tumor, Node, Metastasis. 


\section{Chapter 5}

The minimum duration of the physical exercise training program was nine weeks and the maximum duration was 17 weeks, with mean \pm SD duration of $11.8 \pm 3.0$ weeks, dependent on the planning for surgery. Out of the 13 patients, nine (69.2\%) were able to follow the entire program without any adverse events and with a progressive build up (intensity and duration of the exercises). Throughout the program, mean \pm SD duration of the training session increased with $23.3 \pm 7.2 \%$. Training intensity at the ergometer and treadmill increased with $72.6 \pm 38.9 \%$ and with $24.7 \pm 18.0 \%$, respectively, whereas mean \pm SD training intensity at the leg press and chest press increased with $50.8 \pm 31.5 \%$ and $55.4 \pm 21.9 \%$, respectively.

Total attendance at the training sessions was 198 out of 207 sessions, meaning an attendance rate of $95.7 \%$. Two patients (22.2\%) missed two of 30 training sessions $(6.7 \%)$, because of feeling ill. One patient (11.1\%) missed one of 18 training sessions $(5.6 \%)$ because of a busy schedule that day and one patient $(11.1 \%)$ missed four of 34 training sessions $(11.1 \%)$, because he had to undergo a percutaneous coronary intervention in the same period as the nCRT. Five patients $(55.6 \%)$ did not miss any training sessions. Hence, the mean \pm SD percentage of missed training sessions was 3.4 $\pm 4.4 \%$. Patients were satisfied and during the training sessions Borg scores of 12,13 or 14 were achieved in all individuals. Leg muscle strength could not be measured eight weeks postoperatively, because of the abdominal wound. Moreover, one of nine patients (11.1\%) did not have T2 measurements, because surgery was brought forward. For two of nine patients $(22.2 \%)$, it was not possible to train on heart rate during the first week of the program, because they were on a beta blocker. In these patients the Borg scale was used from the start of the training program. All patients rated the content of the training sessions, the guidance during the training sessions, and the sport facilities with high mean scores (1-10) of 9, 9 and 8, respectively.

\section{Preliminary effectiveness}

\section{Functional exercise capacity}

Preoperatively, six of nine patients (67.0\%) improved their walking distance at the 6MWT between baseline and T1 (mean \pm SD distance of $509.6 \pm 124.5 \mathrm{~m}$ versus $528.7 \pm 99.7$ $\mathrm{m}$, respectively; not statistically significant; $P=0.526)$. Between $\mathrm{T} 1$ and $\mathrm{T} 2$, six of eight (75.0\%) improved their walking distance (mean \pm SD distance of $528.7 \pm 99.7 \mathrm{~m}$ versus $555.6 \pm 101.7 \mathrm{~m}$, respectively; not statistically significant; $P=0.202$ ). At $T 2$, seven of eight $(87.5 \%)$ patients walked a longer distance compared to baseline (mean \pm SD distance of $555.6 \pm 101.7 \mathrm{~m}$ versus $509.6 \pm 124.5 \mathrm{~m}$, respectively; not statistically significant; $P=0.075$ ). This equals a mean increase of $9.0 \%$. Postoperatively (T3, eight weeks after surgery), five of five patients (100.0\%) had a decreased walking distance (mean \pm SD distance of $540.8 \pm 147.9 \mathrm{~m}$ ), of which three of five patients $(60.0 \%)$ scored below baseline level, see Figure $2 a$. 


\section{Muscle strength}

Preoperatively, seven of nine patients $(77.8 \%)$ improved their leg muscle strength between baseline and T1 (mean \pm SD strength of $104.0 \pm 32.3 \mathrm{~kg}$ versus $120.7 \pm 34.0$ $\mathrm{kg}$, respectively; $P=0.035)$. Between $\mathrm{T} 1$ and $\mathrm{T} 2$, six of eight $(75.0 \%)$ patients improved their leg muscle strength (mean \pm SD strength of $120.7 \pm 34.0 \mathrm{~kg}$ versus $144.8 \pm 45.6$ $\mathrm{kg}$, respectively; $P=0.019)$. At T2, eight of eight patients (100.0\%) improved their leg muscle strength statistically significant compared to baseline (mean \pm SD strength of $144.8 \pm 45.6 \mathrm{~kg}$ versus $104.0 \pm 32.3 \mathrm{~kg}$, respectively; $P<0.001)$. This equals a mean improvement of $39.2 \%$. See also Figure $2 \mathrm{~b}$.

Preoperatively, seven of nine patients (77.8\%) improved their arm muscle strength between baseline and T1 (mean \pm SD strength of $36.1 \pm 11.0 \mathrm{~kg}$ versus $42.1 \pm 13.7 \mathrm{~kg}$, respectively; not statistically significant; $P=0.158$ ). Between $\mathrm{T} 1$ and $\mathrm{T} 2$, six of eight patients (75.0\%) improved their arm muscle strength (mean \pm SD strength of $42.1 \pm$ $13.7 \mathrm{~kg}$ versus $48.7 \pm 13.8 \mathrm{~kg}$, respectively; not statistically significant; $P=0.098$ ). At T2, eight of eight patients (100.0\%) improved their arm muscle strength compared to baseline (mean \pm SD strength of $48.7 \pm 13.8 \mathrm{~kg}$ versus $36.1 \pm 11.0 \mathrm{~kg}$, respectively; statistically significant; $P=0.002$ ). This is a mean increase of $34.9 \%$. Postoperatively (T3), arm muscle strength decreased in five of five patients (100\%) (mean \pm SD strength of $40.4 \pm 11.3 \mathrm{~kg}$ ), of which no one showed a decrease below baseline values, see Figure $2 \mathrm{~b}$. Figure 3 shows the preliminary effectiveness of the physical exercise training program during nCRT on fatigue and perceived quality of life.

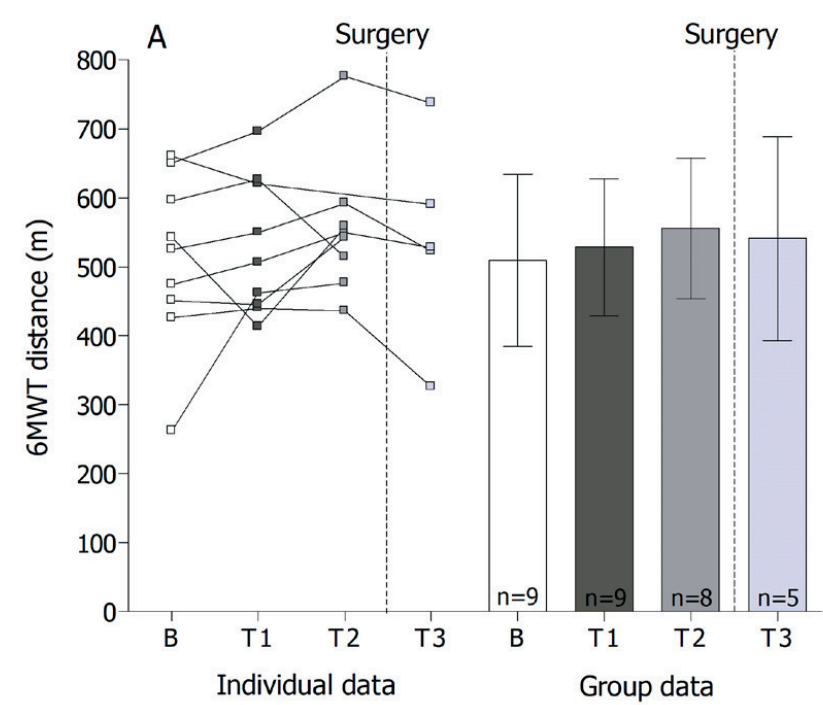

Figure 2a. Preliminary effectiveness of the physical exercise training program during nCRT on physical fitness, both on the individual (lines) and group (bars) level, at baseline (B), after 5 weeks of training (T1), after 10 weeks of training (T2), and 8 weeks after surgery (T3). Graph A: functional exercise capacity (6MWT distance). Abbreviations: $6 \mathrm{MWT}=$ six-minute walk test; $\mathrm{nCRT}=$ neoadjuvant chemoradiotherapy; $\mathrm{SD}=$ standard deviation. 

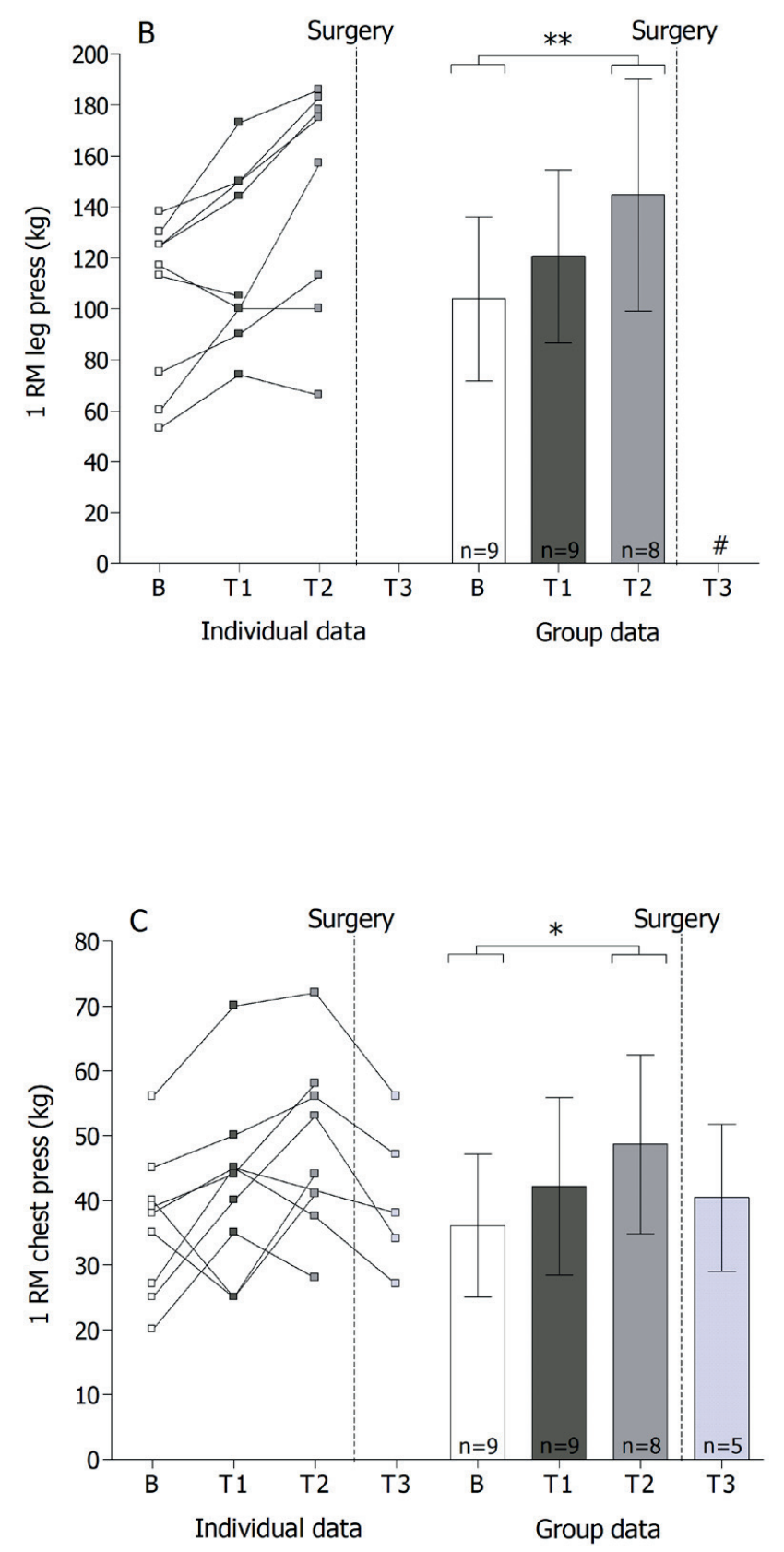

Figure $\mathbf{2 b}$. Preliminary effectiveness of the physical exercise training program during $\mathrm{nCRT}$ on physical fitness, both on the individual (lines) and group (bars) level, at baseline (B), after 5 weeks of training (T1), after 10 weeks of training (T2), and 8 weeks after surgery (T3). Graph B: muscle strength of the legs (1RM at the leg press); graph C: muscle strength of the arms (1RM at the chest press). Abbreviations: $1 \mathrm{RM}=$ one-repetition maximum; $\mathrm{nCRT}=$ neoadjuvant chemoradiotherapy; $\mathrm{SD}=$ standard deviation. $* P<0.01 ; * * \quad P<0.001$.

\# Leg muscle strength could not be measured eight weeks postoperatively due to the abdominal wound. 

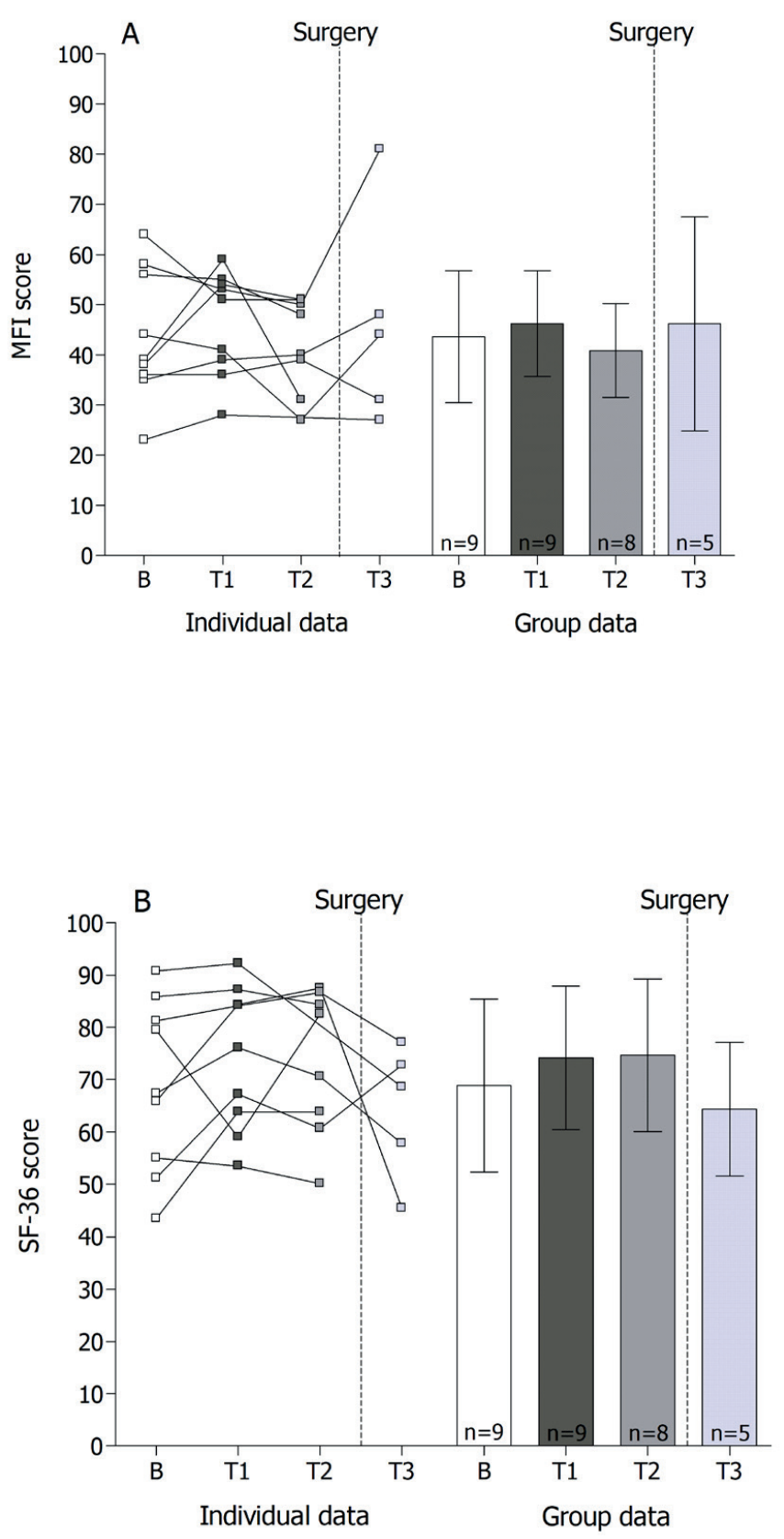

Figure 3. Preliminary effectiveness of the physical exercise training program during nCRT on fatigue and perceived quality of life, both on the individual (lines) and group (bars) level, at baseline (B), after 5 weeks of training (T1), after 10 weeks of training (T2), and 8 weeks after surgery (T3). Group data are expressed as mean \pm SD. Graph A: fatigue (MFI score); graph B: perceived quality of life (SF-36 score). Abbreviations: MFI=multidimensional fatigue index; $\mathrm{nCRT}=$ neoadjuvant chemoradiotherapy; $\mathrm{SD}=$ standard deviation; SF-36=short-form 36 health survey. 


\section{DISCUSSION}

This is the first pilot study that investigated the feasibility and preliminary effectiveness of a supervised outpatient physical exercise training program for patients with locally advanced resectable rectal cancer during nCRT. Although the physical exercise program was safe, feasible and able to prevent an often seen decline in physical fitness during $\mathrm{nCRT}$ in the patient who completed the program, it appeared to be difficult to include all eligible patients in the program.

The current study demonstrated that only a significant part of the patients with rectal cancer was willing and able to participate in the physical exercise training program during nCRT. Their attendance rate was $95.7 \%$, without any adverse events. Of the 20 eligible patients, seven $(35.0 \%)$ were not able or not willing to participate and during the program, four of 13 patients (30.8\%) dropped out. Six of 20 eligible patients preferred nCRT without participating in the physical exercise training program. Reasons mentioned for this were; a busy day schedule $(n=1)$, disease impact $(n=3)$ and no need for participation $(n=2)$. For one patient, participation was contraindicated due to a cardiovascular comorbidity. Four of 13 patients that started with the physical exercise training program dropped out due to side effects (radiation burns) of the nCRT $(n=1)$, already existing knee problems $(n=1)$, and an increase in perceived fatigue $(n=2)$. It is unknown whether the increase in perceived fatigue can be explained by the physical exercise training program alone, as other studies demonstrate a positive effect of training on fatigue in these patients [26]. The remaining nine patients completed the program and corresponding evaluations. For these patients, the supervised physical exercise training program during nCRT was safe and feasible. Patients adhered to the program with progressive overload and did not report discomfort or adverse effects. This is in line with revenues from a recent other type of study from West et al. [3]. In the latter study, 22 patients with locally advanced rectal cancer participated in a six-week preoperative physical exercise training program after completing nCRT. This program was also found to be safe and feasible ( $96 \%$ adherence, with no adverse events). Likewise in breast cancer patients, a physical exercise training program during neoadjuvant chemotherapy ( $\mathrm{nCT}$ ) has been reported to be safe and feasible. Rao et al. [27] performed a randomized pilot trial to investigate the effects of physical exercise training (boot camp) in patients with breast cancer throughout their $\mathrm{nCT}$ treatment $(\mathrm{n}=10)$. All patients in the exercise group $(n=5)$ completed the program and all attended $>80 \%$ of the advised exercise sessions. The combined study of Jones et al. [28] and Hornsby et al. [29] completed a randomized pilot trial in breast cancer patients receiving $\mathrm{nCT}(\mathrm{n}=20)$, in which the exercise group $(n=10)$ performed a moderate-to-high intensity aerobic exercise training program during $\mathrm{nCT}$. One patient in the exercise group did not complete the physical exercise training program, because of deep vein thrombosis and pulmonary embolism. 
Furthermore, the patients appreciated the training sessions and were satisfied about the program.

Participating in the moderate-intensity physical exercise training program during nCRT in the current study did not only prevent the expected decline in physical fitness due to nCRT. It led to a significant increase in leg muscle strength (+39.2\%) and arm muscle strength (+34.9) after ten weeks of training with a slight, non-significant, increase in functional exercise capacity throughout nCRT (+9.0\%). Fatigue and quality of life remained relatively stable throughout the program. Three studies of West et al. [3-5] demonstrated that nCRT acutely reduces objectively measured cardiorespiratory fitness significantly in patients awaiting rectal cancer surgery (a reduction in $\mathrm{VO}_{2}$ at the ventilatory threshold of $12.4 \%$ [4], $19.5 \%$ [5], and $14.2 \%$ [3], and a reduction in $\mathrm{VO}_{2 \text { peak }}$ of $7.7 \%$ [4], 21.4\% [5], and 15.3\% [3]). A study of Jack et al. [10] in patients undergoing oesophagogastric cancer surgery also showed that nCT before surgery significantly reduces objectively measured cardiorespiratory fitness (a reduction in $\mathrm{VO}_{2}$ at the ventilatory threshold of $15.2 \%$ and in $\mathrm{VO}_{2 \text { peak }}$ of $12.0 \%$ ). The structured preoperative physical exercise training program immediately post-nCRT in rectal cancer patients as described by West et al. [3] was able to return cardiorespiratory fitness to baseline levels in six weeks (an increase in $\mathrm{VO}_{2}$ at the ventilatory threshold of $20.4 \%$ and in $\mathrm{VO}_{2 \text { peak }}$ of $16.9 \%$ ). The current pilot study is the first study that demonstrated that a moderateintensity physical exercise training program during nCRT in rectal cancer patients may prevent a decline in physical fitness caused by nCRT. Also in breast cancer patients, objectively measured cardiorespiratory fitness increased in the group that performed moderate-to-high intensity aerobic exercise training during $\mathrm{nCT}$ (an increase in $\mathrm{VO}_{2 \text { peak }}$ of $13.3 \%$ ) $[28,29]$. On the other hand, patients in the control group showed a reduction in cardiorespiratory fitness after nCT (a reduction in $\mathrm{VO}_{2 \text { peak }}$ of $8.6 \%$ ), in which the between-group difference was significant $[28,29]$.

Based on the current study results, it seems possible for patients with rectal cancer to maintain their physiological reserve by training during nCRT. A higher level of physical fitness is associated with better postoperative outcomes [11-15] and also may facilitate a faster functional recovery after surgery [30]. Our results are in line with a recently published systematic review that evaluated the evidence in support of preoperative exercise training in cancer patients undergoing the dual challenges of neoadjuvant therapy and surgery [31]. The authors concluded that supervised moderate-to-severe intensity exercise training significantly improved cardiorespiratory fitness in these patients. Nowadays, it is clear that a poor cardiorespiratory fitness is associated with a complicated postoperative period. Exercise training during nCRT in rectal cancer patients can prevent the expected decline in physical fitness caused by nCRT to improve surgical outcomes. Postoperative data in the current study show a decline in physical fitness compared to the preoperative data in all patients; however, mean values for functional exercise capacity and arm muscle strength were still above baseline values, which may 


\section{Chapter 5}

facilitate a faster functional recovery. Interesting facts that wait to be provided with hard evidence from a full blown trial.

\section{Lessons learned}

Only 13 of the 20 eligible patients started with the training program, which might be attributable to the location of the training sessions, as well as to the lack of adequate patient education by physicians about the effects and importance of physical exercise training during nCRT. During the first 5.5 weeks, training sessions were combined with the radiotherapy sessions in our hospital which was well-appreciated by the patients. For the remaining preoperative period, patients had to visit the hospital for the training sessions alone, which might have been an extra burden to participate. The participation rate might have been higher if the exercise program was delivered at the patient's own living situation, as there is evidence that (frail) elderly are less likely to participate in a clinic-based exercise program than they are in a home-based exercise program. Moreover, patients should be stimulated and adequately informed by their physician regarding the importance of physical fitness throughout the course of their treatment

In conclusion, the current study revealed that a supervised outpatient physical exercise training program for patients with locally advanced resectable rectal cancer during nCRT is feasible for a large part of the patients, safe and seems effective in the prevention of an often seen decline in physical fitness during nCRT. Fatigue and quality of life remained relatively stable during the program. Because of its potential effects, we should look for means to have more patients to profit from such an approach, hand in hand with an approach to proof its cost-effectiveness in a larger study. 


\section{REFERENCES}

1. The Netherlands Comprehensive Cancer Organisation (IKNL), Cijfers over kanker, The Netherlands.http://www.cijfersoverkanker.nl/selecties/Dataset_1/img55b89450bdfdb, 2015 (accessed December 2015).

2. Dutch Institute for Clinical Auditing (DICA) Leiden, DICA Jaarrapportage 2014: DSCA. http://www.clinicalaudit.nl/jaarrapportage/2014/dsca.html, (accessed December 2015).

3. West MA, Loughney L, Lythgoe $D$, et al., Effect of prehabilitation on objectively measured physical fitness after neoadjuvant treatment in preoperative rectal cancer patients: a blinded interventional pilot study, Br J Anaesth. 114 (2015) 244-251.

4. West MA, Loughney $\mathrm{L}$, Barben $\mathrm{CP}$, et al., The effects of neoadjuvant chemoradiotherapy on physical fitness and morbidity in rectal cancer surgery patients, Eur J Surg Oncol. 40 (2014) 1421-1428.

5. West MA, Loughney L, Lythgoe $D$, et al., The effect of neoadjuvant chemoradiotherapy on wholebody physical fitness and skeletal muscle mitochondrial oxidative phosphorylation in vivo in locally advanced rectal cancer patients-an observational pilot study, PLoS One. 9 (2014) 1-15.

6. Bosset JF, Collette L, Calais G, et al., Chemotherapy with preoperative radiotherapy in rectal cancer, N Engl J Med. 355 (2006) 1114-1123.

7. Chau I, Brown G, Cunningham D, et al., Neoadjuvant capecitabine and oxaliplatin followed by synchronous chemoradiation and total mesorectal excision in magnetic resonance imaging-defined poor-risk rectal cancer, J Clin Oncol. 24 (2006) 668-674.

8. Swellengrebel HA, Marijnen CA, Verwaal VJ, et al., Toxicity and complications of preoperative chemoradiotherapy for locally advanced rectal cancer, Br J Surg. 98 (2011) 418-426.

9. Windsor PM, Potter J, McAdam K, et al., Evaluation of a fatigue initiative: information on exercise for patients receiving cancer treatment, Clin Oncol (R Coll Radiol). 21 (2009) 473-482.

10. Jack $S$, West MA, Raw $D$, et al., The effect of neoadjuvant chemotherapy on physical fitness and survival in patients undergoing oesophagogastric cancer surgery, Eur J Surg Oncol. 40 (2014) 13131320.

11. Snowden CP, Prentis J, Jacques B, et al., Cardiorespiratory fitness predicts mortality and hospital length of stay after major elective surgery in older people, Ann Surg. 257 (2013) 999-1004.

12. West MA, Lythgoe $\mathrm{D}$, Barben $\mathrm{CP}$, et al., Cardiopulmonary exercise variables are associated with postoperative morbidity after major colonic surgery: a prospective blinded observational study, $\mathrm{Br}$ ] Anaesth. 112 (2014) 665-671.

13. West MA, Parry MG, Lythgoe $D$, et al., Cardiopulmonary exercise testing for the prediction of morbidity risk after rectal cancer surgery, Br J Surg. 101 (2014) 1166-1172.

14. Levett DZ, Grocott MP, Cardiopulmonary exercise testing for risk prediction in major abdominal surgery, Anesthesiol Clin. 33 (2015) 1-16.

15. Hennis PJ, Meale PM, Grocott MP, Cardiopulmonary exercise testing for the evaluation of perioperative risk in non-cardiopulmonary surgery, Postgrad Med J. 87 (2011) 550-557.

16. Older P, Smith R, Experience with the preoperative invasive measurement of haemodynamic, respiratory and renal function in 100 elderly patients scheduled for major abdominal surgery, Anaesth Intensive Care. 16 (1988) 389-395.

17. Older P, Hall A, Clinical review: how to identify high-risk surgical patients, Crit Care. 8 (2004) 369372.

18. Rödel C, Graeven U, Fietkau R, et al., Oxaliplatin added to fluorouracil-based preoperative chemoradiotherapy and postoperative chemotherapy of locally advanced rectal cancer (the German CAO/ARO/AIO-04 study): final results of the multicentre, open-label, randomised, phase 3 trial, The Lancet oncology. 16 (2015) 979-989. 


\section{Chapter 5}

19. Lin KY, Shun SC, Lai YH, et al., Comparison of the effects of a supervised exercise program and usual care in patients with colorectal cancer undergoing chemotherapy, Cancer Nurs. 37 (2014) E21-29.

20. Glasziou P, Irwig L, Mant D, Monitoring in chronic disease: a rational approach, BMJ. 330 (2005) 644648.

21. ATS Committee on proficiency standards for clinical pulmonary function laboratories, ATS statement: guidelines for the six-minute walk test, Am J Respir Crit Care. 166 (2002) 111-117.

22. Grimbsy O, Rivard J, Science, Theory and clinical application in orthopaedic manual physical therapy: applied science and theory, Vol 1, Academy of Graduate Physical Therapy, Inc., Taylorsville, 2008.

23. Courneya KS, Exercise interventions during cancer treatment: biopsychosocial outcomes, Exerc Sport Sci Rev. 29 (2001) 60-64.

24. McDowell I, Measuring health: a guide to rating scales and questionnaires, 3rd ed, Oxford University Press, 2006.

25. Maas M, Beets-Tan RG, Lambregts DM, et al., Wait-and-see policy for clinical complete responders after chemoradiation for rectal cancer, J Clin Oncol. 29 (2011) 4633-4640.

26. Meneses-Echavez JF, Gonzalez-Jimenez E, Ramirez-Velez R, Effects of supervised multimodal exercise interventions on cancer-related fatigue: systematic review and meta-analysis of randomized controlled trials, BioMed Res Int. 2015 (2015) 328636.

27. Rao R, Cruz V, Peng $Y$, et al., Bootcamp during neoadjuvant chemotherapy for breast cancer: a randomized pilot trial, Breast Cancer (Auckl). 6 (2012) 39-46.

28. Jones LW, Fels DR, West M, et al., Modulation of circulating angiogenic factors and tumor biology by aerobic training in breast cancer patients receiving neoadjuvant chemotherapy, Cancer Prev Res (Phila). 6 (2013) 925-937.

29. Hornsby WE, Douglas PS, West MJ, et al., Safety and efficacy of aerobic training in operable breast cancer patients receiving neoadjuvant chemotherapy: a phase II randomized trial, Acta Oncol. 53 (2014) 65-74.

30. Gillis C, Li C, Lee L, et al., Prehabilitation versus rehabilitation: a randomized control trial in patients undergoing colorectal resection for cancer, Anesthesiology. 121 (2014) 937-947.

31. Loughney L, West MA, Kemp GJ, et al., Exercise intervention in people with cancer undergoing neoadjuvant cancer treatment and surgery: A systematic review, Eur J Surg Oncol. 42 (2016) 28-38. 




\section{Chapter $\supset$ supplement}

\section{Reply to: Exercising patient-centeredness in prehabilitation programs}

Aniek F. Heldens

Bart C. Bongers

Judith de Vos-Geelen

Nico L. van Meeteren

Antoine F. Lenssen

European Journal of Surgical Oncology 2017;43:511-512. 

Augmented collective understanding of human nature facilitates stepwise improvement of health care interventions, be it for instance pharmacological or psychological interventions. The very same holds particularly true for interventions like prehabilitation with help of physical exercise training. Underlying here are recent psychobiological understandings combined with critical reflections of leading scientists in new inspiring concepts like Predictive, Preventive, Personalized, and Participatory (P4) health [1]. This concept and others pay attention to the uniqueness of individual patients and, for the most based on this uniqueness, the need for individual patient-centeredness. The highly appreciated response of colleague Wright adds up to this literature and extends these developments towards the domain of physical exercise training programs. Wright holds, in line with for example Hoogeboom et al. [2], a plea for the uniqueness of individual patients, as well as for patient-centeredness in exercise prehabilitation in order to improve patient adherence, especially for the most vulnerable. A plea also for the reinvention of physical exercise training. With respect to this plea, we formerly introduced the concept of "therapeutic validity" [3], as an important add on for randomized controlled trials and in systematic reviews that, up till then, advised and evaluated methodological quality rather than the content of the physical exercise training program itself. Physical exercise training programs in randomized controlled trials often lack overload, include the younger and more healthy subgroup of patients, and fail to bring out the full potential of their patients [3]. The hereto developed CONTENT-scale urged therapists and researchers to pay more attention to the content of a physical exercise training program and emphasized that this should be tailored to the individual patient, rather than having a "one size fits all" approach.

In our pilot study [4], patients were offered physical exercise training during the period in which they underwent neoadjuvant chemoradiotherapy (nCRT). This was actually the first study that investigated the feasibility and preliminary effectiveness of this approach to prevent the often in clinical practice seen deterioration of physical fitness during nCRT. Because the period of nCRT is often a physically and mentally demanding period, we specifically chose for an in-hospital supervised physical exercise training program instead of a home-based program - where training sessions at our physical therapy outpatient clinic could be combined with the clinic visits for the radiation schedule of the patients. A strategy that was highly appreciated by the participating patients [4]. Our study showed favorable results on feasibility, safety, adherence, and effectiveness in nine of thirteen patients (69\%) who completed the preoperative physical exercise training program during nCRT. Four patients (31\%) dropped out due to several reasons [4]. For these patients it might have been possible to participate in a preoperative physical exercise training program in the period between nCRT and surgery, such as described by West et al. [5]. The seven out of twenty eligible patients (35\%) that refused to participate, might have been willing to participate when a more patient-centered and individually tailored physical exercise training program was provided at home. A program 
that includes the patient especially, as well as their relatives, and (in)formal caregivers. Together they should aim to preserve or even improve physical fitness for patients exposed to the challenge(s) of nCRT and/or major abdominal surgery to improve treatment outcome, preferably via a multimodal approach including psychosocial and nutritional counseling - if necessary on the base of diagnostics - as well. In fact, prevention of a complicated postoperative course and a rapid return to adequate performance of (instrumental) activities of daily living in patients is mandatory and essential to preserve independent functional performance and perceptions of quality of life [6]. This may also lead to an improvement in health-care quality experiences, and even lead to further cost-savings.

An important next step is indeed to improve the participation rate and adherence of patients in the exercise prehabilitation program. Firstly, patients, their relatives, and their (in)formal caregivers should be sufficiently educated and motivated about the significance of physical activity and physical fitness before and after surgery for adequate post-surgical functional recovery. Secondly, the program should be more patientcentered, meaning personalized to the short- and long-term goals, needs, preferences, and physical and mental potentials of the patient. Thirdly, the physical exercise program should be planned, structured, executed, and monitored with the patient, preferably within his or her own living situation, as there is evidence $[7,8]$ that vulnerable patients are less likely to participate in a clinic-based exercise program than they are in a homebased physical exercise training program. Best would also be that the revenues of the program are monitored by patients themselves, where and when necessary supported by their relatives and their (in)formal caregivers, as well as by their physical therapist by frequently using functional tests to direct and titrate exercise dosage [9]. This will probably improve their involvement and motivation and thereby adherence and satisfaction. Preferably, the patient decides, well informed by the physical therapist, the anesthesiologist, and the surgeon, and also with respect to the medical condition, when he or she is indeed fit to operate on. Thereupon the decision of planning for surgery is best made. 


\section{REFERENCES}

1. Hood L, Friend SH, Predictive, personalized, preventive, participatory (P4) cancer medicine, Nat Rev Clin Oncol. 8 (2011) 184-187.

2. Hoogeboom TJ, Kittelson AJ, van der Sluis G, et al., Evidence-based practice in physiotherapy: time to involve the patient? Physiotherapy. 100 (2014) 363-364.

3. Hoogeboom TJ, Oosting E, Vriezekolk JE, et al., Therapeutic validity and effectiveness of preoperative exercise on functional recovery after joint replacement: a systematic review and meta-analysis, PloS One. 7 (2012) e38031.

4. Heldens AF, Bongers BC, de Vos-Geelen J, et al., Feasibility and preliminary effectiveness of a physical exercise training program during neoadjuvant chemoradiotherapy in individual patients with rectal cancer prior to major elective surgery, Eur J Surg Oncol. 42 (2016) 1322-1330.

5. West MA, Loughney L, Lythgoe $D$, et al., Effect of prehabilitation on objectively measured physical fitness after neoadjuvant treatment in preoperative rectal cancer patients: a blinded interventional pilot study, Br J Anaesth. 114 (2015) 244-251.

6. Dronkers JJ, Witteman BJ, van Meeteren NL, Surgery and functional mobility: doing the right thing at the right time, Tech Coloproctol. 20 (2016) 339-341.

7. Oosting $\mathrm{E}$, Jans MP, Dronkers JJ, et al., Preoperative home-based physical therapy versus usual care to improve functional health of frail older adults scheduled for elective total hip arthroplasty: a pilot randomized controlled trial, Arch Phys Med Rehabil. 93 (2012) 610-616.

8. Hulzebos EH, van Meeteren NL, van den Buijs BJ, et al., Feasibility of preoperative inspiratory muscle training in patients undergoing coronary artery bypass surgery with a high risk of postoperative pulmonary complications: a randomized controlled pilot study, Clin Rehabil. 20 (2006) 949-959.

9. Glasziou P, Irwig L, Mant D, Monitoring in chronic disease: a rational approach, BMJ. 330 (2005) 644648. 



\section{Chapter}

\section{A one-group pre-test post-test pilot study to evaluate a supervised home-based prehabilitation program for high-risk patients undergoing major elective abdominal surgery: a study protocol}

Aniek F. Heldens

Nico L. van Meeteren

Christel A. van Beijsterveld Jarno Melenhorst

Mariëlle Coolsen

Marcel den Dulk

Cornelius H. Dejong

Laurents P. Stassen

Wolfgang F. Buhre

Bart C. Bongers

Submitted for publication. 


\section{Chapter 6}

\section{ABSTRACT}

\section{Background.}

The increasing aging population leads to a rise in the incidence of gastrointestinal malignancies. Here elective abdominal resection is the main treatment option, with significant morbidity and mortality rates. A poor aerobic capacity, indicating a reduced physiological reserve, increases the risks for a complicated postoperative course and prehabilitation is an option to reverse the risks. Evidence regarding the feasibility of a home-based prehabilitation program for high-risk patients undergoing major abdominal surgery is scarce. Therefore, the primary objective of this study is to assess feasibility of a four-week home-based prehabilitation program in high-risk patients scheduled for elective gastrointestinal resection.

\section{Methods.}

In this feasibility study, with a one group pre-test post-test design, especially high-risk patients will execute additional measurements concerning the feasibility and individual response to a home-based prehabilitation program. Before and after the prehabilitation program, the following measurements will be performed: a venipuncture, a cardiopulmonary exercise test, and filling out three questionnaires (nutritional status, quality of life, and health status). During the program, patients fill out a physical activity diary. Furthermore, data on adherence and compliance will be recorded. After the fourweek prehabilitation program, the patients will fill out an appreciation questionnaire.

\section{Discussion.}

The hypothesis for this study is that home-based personalized prehabilitation is feasible, based on robust program adherence/compliance, with no adverse events, with a high patient appreciation. The results of this study should also prepare for the next step to personalize and specify the currently used preventive and monitoring ingredients of the prehabilitation program of the high-risk patients.

Trial registration. NL65596.068.18 


\section{INTRODUCTION}

As the aging population is growing, the incidence of gastrointestinal malignancies will also rise. Colorectal cancer is the third most common cancer diagnosis in the Netherlands (incidence in 2018: 14,090) [1]. Additionally, 791 new cases of hepatic cancer and 2,348 cases of pancreatic cancer were registered in the Netherlands in 2018 [1]. For a large part of the patients, elective abdominal resection is the main treatment option [2]. Major abdominal resection of these malignancies is significantly associated with morbidity and even mortality $[3,4]$.

The cardiopulmonary exercise test (CPET) is the gold standard test to objectively evaluate a patient's preoperative aerobic capacity $[5,6]$. Preoperative aerobic capacity has a consistent positive relation with postoperative outcomes in major abdominal surgery [7,8-10], as patients with a ventilatory anaerobic threshold (VAT) $\leq 11$ $\mathrm{mL} / \mathrm{kg} / \mathrm{min}$ are generally found to be at an increased risk for a complicated postoperative period after major surgery $[5,6]$. Patients with a higher preoperative VAT have an increased ability to adequately cope with the surgery-induced stress, which significantly increases the metabolic demands after surgery and the risk of postoperative complications [7]. Previous studies have shown that alternative exercise tests as the incremental shuttle walk test (iSWT) and the stair-climbing test can also serve as an indicator of postoperative risk in patients undergoing abdominal surgery [11-13]. A study of Moran et al. has shown that the evidence behind field tests to predict postoperative complications is weaker, compared to CPET testing and further research is needed to justify their use [14].

Prehabilitation is based on the principle that structured preoperative interventions provide high-risk patients with a psychophysiological buffer to better withstand the stress of surgery [15-17], which might minimize the risks of the temporary decline of physical functioning following major surgery [18]. Prehabilitation programs have been shown to improve physical fitness before surgery in patients undergoing major abdominal surgery [19-21], of which a recent study in high-risk patients showed that prehabilitation can improve physical fitness before major abdominal resection and subsequently induce beneficial effects on postoperative morbidity and length of hospital stay [22]. The selection of in- and exclusion criteria is diverse, the content and execution of several prehabilitation programs is heterogeneous and mostly groups are targeted whereas personalized seems more profitable. A recent clinical guideline with recommendations for preoperative exercise training in patients opting for non-cardiac surgery emphasizes the importance of a supervised multimodal program with a combination of aerobic training, resistance training and inspiratory muscle training [23]. The frequency, intensity and duration of each exercise component should be tailored to the individual patient, taking their initial level of physical fitness and their personal progress during the program into account. As so, the program should be frequently monitored using performance- 
based tests, evaluated, and adjusted during the preoperative period to achieve maximal effects [24]. Although promising concerning its effectiveness, evidence regarding the feasibility and individual responses to a supervised personalized home-based prehabilitation in high-risk patients undergoing major abdominal surgery is lacking. Additionally, there is not much known about home-based prehabilitation with functional exercises with high frequent monitoring of progression. Therefore, the primary objective of this pilot study is to assess feasibility (adherence/compliance, adverse events, and patient appreciation) of a four-week supervised and personalized home-based prehabilitation program for high-risk patients scheduled for elective colorectal, hepatic, or pancreatic resection. Secondary objective(s) are to evaluate individual responses to the program in terms of physical fitness, immune system responses, nutritional status, self-reported quality of life, and self-reported health status.

\section{METHODS}

\section{Study design}

In this one-group pre-test post-test pilot study, high-risk patients who provide informed consent for additional measurements concerning the feasibility of a supervised and personalized home-based prehabilitation program (approximately four weeks, 12 sessions in total) will be included.

\section{Participants}

Patients scheduled for major elective colorectal, hepatic, or pancreatic resection at the Maastricht University Medical Center (Maastricht UMC+) will be invited to participate in a screening for potential eligibility by a hospital physical therapist as part of usual care. Patients with a low physical fitness will be advised to participate in a usual care prehabilitation program. These patients with a low physical fitness will be informed about the current pilot study, which includes extra measurements before and after the prehabilitation program to evaluate the program's feasibility and individual responses to the program. Patients who perform adequately (sufficient physical fitness, fit for surgery) on the preoperative screening (usual care) are not eligible for participating in the hospital's home-based prehabilitation program, will therefore not be approached for this study, and will receive usual care (advice on the importance of preoperative physical fitness and physical activity in relation to postoperative outcomes, recovery of physical functioning, and quality of life). The inclusion criteria of the study are; $>18$ years of age; scheduled for elective colorectal, hepatic, or pancreatic resection at the Maastricht UMC+; a low physical fitness according to the preoperative screening with the advice to participate in the hospital's prehabilitation program (usual care); willing to participate in the hospital's prehabilitation program (usual care); providing informed consent to 
participate; and classified as being at risk for a complicated postoperative period (VAT $\leq 11 \mathrm{~mL} / \mathrm{kg} / \mathrm{min}$ at the pre-prehabilitation CPET). Patients that require acute (emergency) surgery, as well as those with contraindications to physical exercise training or those unable to cooperate with the testing procedures (e.g., insufficient understanding of the Dutch language) will be excluded.

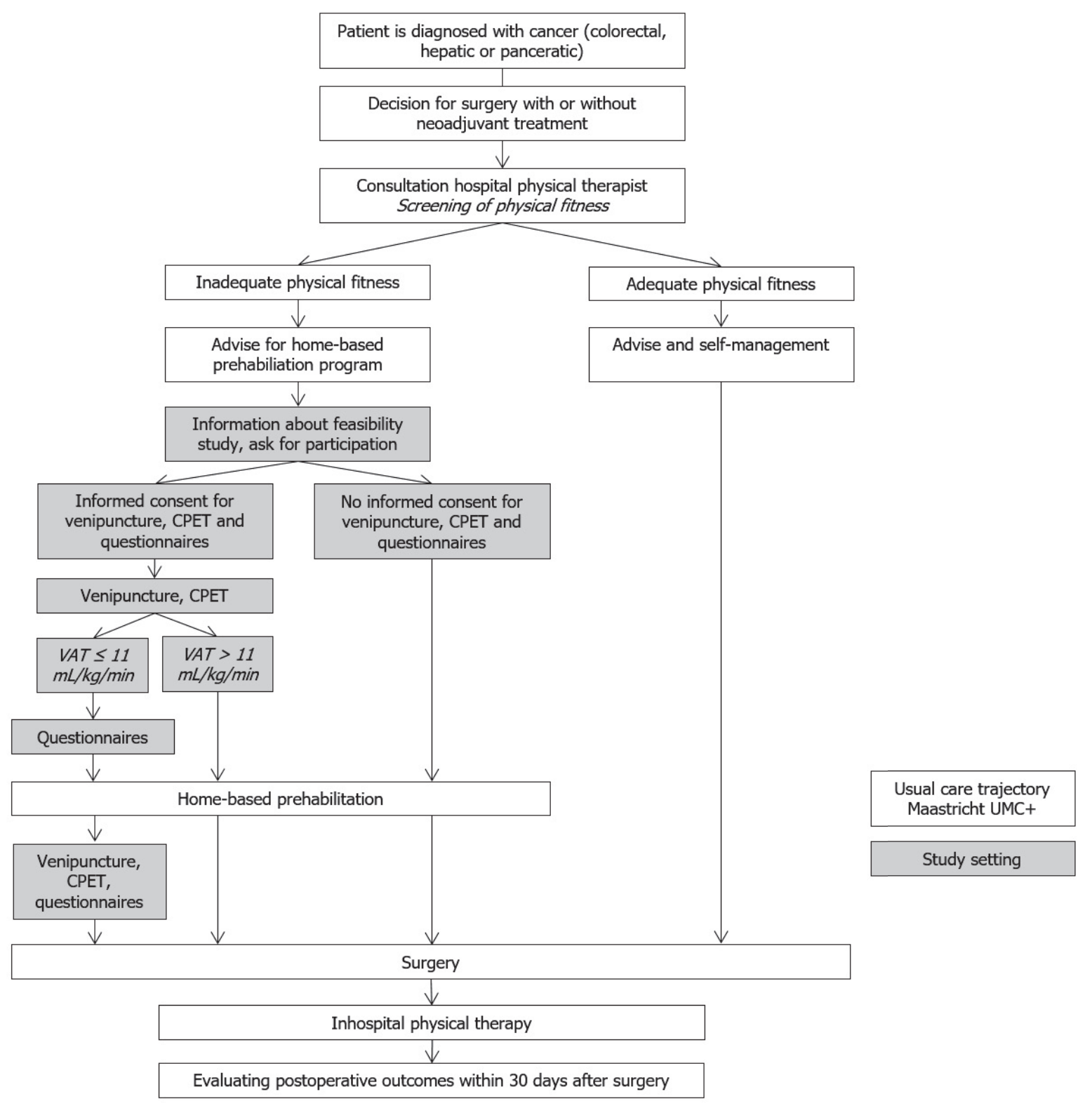

Figure 1. Flowchart of the procedures this pilot study. Abbreviations: $C P E T=$ cardiopulmonary exercise test; UMC+=University Medical Center; VAT=Ventilatory anaerobic threshold. 


\section{Chapter 6}

\section{Recruitment}

Patients with a low physical fitness at the preoperative screening by the hospital physical therapist will be advised to participate in a prehabilitation program (usual care). These patients are potentially eligible for study participation and will be informed about this pilot study. They will receive a patient information letter, legitimized by the ethical committee, that explains the aims and expectations of the study, as well as the risks and benefits of participation (see Figure 1). Interested patients will be contacted a few days later by phone to answer their questions and ask for oral informed consent. When patients provide oral informed consent, an appointment for the first study visit will be planned. At this study visit, written informed consent will be obtained before commencing any study-related procedures. Participants can leave the study at any time for any reason without any consequences. The investigators can decide to withdraw a participant from the study for urgent medical reasons. When a patient decides not to participate in this (or any other) study, this will not interfere the patient-physician relationship and the patient will participate in care as usual (e.g., supervised home-based prehabilitation, postoperative physical therapy treatment).

\section{Intervention and measurement procedures}

The intervention consists of extra measurements before and after the prehabilitation program to evaluate the program's feasibility and individual responses to the program. Table 1 shows an overview of the different usual care and study assessments over time.

\section{Pre-prehabilitation measurements}

After written informed consent, a venipuncture and a maximal CPET will be performed to check for study eligibility. In case a patient's VAT is $\leq 11 \mathrm{~mL} / \mathrm{kg} / \mathrm{min}$, the patient is considered a high-risk patient, and when the CPET also reveals no contraindications to physical exercise training, the patient will be definitely included in the study. Hereafter, three questionnaires (nutritional status, quality of life, and health status) will be completed. Patients who have a VAT $>11 \mathrm{~mL} / \mathrm{kg} / \mathrm{min}$ at the CPET are not eligible for this study and will receive usual care (e.g., supervised home-based prehabilitation, postoperative physical therapy treatment).

\section{Anthropometry}

Before the pre-prehabilitation CPET, the patient's body mass (determined to the nearest $0.1 \mathrm{~kg}$ ) and body height (determined to the nearest $0.5 \mathrm{~cm}$ ) will be measured with an electronic scale (Seca 803, Seca, Hamburg, Germany) and using a stadiometer (Seca, Hamburg, Germany), respectively. The measurement of body mass will continue on a weekly base during the prehabilitation program (at the patient's home) as well. 


\section{Immune system functioning}

To assess the individual response of the prehabilitation program on the immune system, blood samples will be drawn before the prehabilitation program. Blood samples will be collected prior to the CPET to determine IL-6, IL-8, IL-10, IL-12, CRP, and TNF-a levels to assess within-subject changes in immune system functioning. The function of the immune system depends mostly on interleukins, and CRP is important for the function of the immune system, as CRP levels rise in response to inflammation.

TNF-a are proteins that are expressed by immune cells and regulate the immune response and inflammation. All blood samples will be obtained at the Maastricht UMC+ by well-trained nurses qualified for this task. The sample will be drawn from a superficial vein in the forearm using a venipuncture. Blood samples will be centrifuged and plasma will be stored at $-80{ }^{\circ} \mathrm{C}$ until assayed for future analysis. Blood samples will be stored, for a maximum of fifteen years, in the biobank of the Maastricht UMC+.

\section{Maximal cardiopulmonary exercise test}

Participants will perform an incremental CPET up to maximal exertion in upright position on an electronically braked cycle ergometer (Lode Corival Rehab, Lode BV, Groningen, the Netherlands). A medical doctor is stand-by in case of emergency and the CPET will be performed by an experienced clinical exercise physiologist. Seat height will be adjusted to the participant's leg length. Before commencing the CPET, forced vital capacity and forced expiratory volume in one second will be obtained from maximal flowvolume curves (Vyntus CPX, CareFusion, Hoechberg, Germany) according to ATS/ERS standards [25]. Subsequently, baseline cardiopulmonary values will be assessed during a two-minute rest period while seated at the cycle ergometer, where after the patient performs a three-minute warm-up phase that consist of unloaded cycling. After the warm-up, the work rate will be increased by constant increments of 5, 10, or $15 \mathrm{~W} / \mathrm{min}$, depending on the patient's subjective physical fitness level and aimed at reaching a maximal effort within eight to twelve minutes. Throughout the CPET, patients have to maintain a pedaling frequency between 60 and 80 revolutions/min. The protocol continues until the patient's pedaling frequency falls definitely $\leq 60$ revolutions/min, despite strong verbal encouragement, or when the patient met the criteria for exercise termination before symptom limitation as proposed in the ATS/ACCP statement on cardiopulmonary exercise testing [26].

During the CPET, patients breath through a facemask (Hans Rudolph, Kansas City, MO, USA) connected to an ergospirometry system (Vyntus CPX, CareFusion, Hoechberg, Germany) that will be calibrated for respiratory gas analysis measurements (ambient air and a gas mixture of $16 \%$ oxygen and $5 \%$ carbon dioxide) and volume measurements (three-liter syringe). Expired gas will pass through a flow meter (Triple $\mathrm{V}$ volume transducer), an oxygen analyzer, and a carbon dioxide analyzer. The flow meter and gas analyzers will be connected to a computer, which calculates breath-by-breath minute 
ventilation, oxygen uptake, carbon dioxide production, and the respiratory exchange ratio averaged at ten-second intervals. Heart rate will be measured by continuous twelve-lead electrocardiography. A test will be considered to be at or near the maximal level when participants show clinical signs of intense effort (e.g., unsteady biking, sweating, and clear unwillingness to continue exercising despite strong encouragement), are unable to maintain the required pedaling speed, and when at least one of the following criteria is met: a heart rate at peak exercise of $>95 \%$ of predicted (predicted peak heart rate [beats $/ \mathrm{min}]=210-(0.8 \times$ age $[$ years] $))$ or a respiratory exchange ratio at peak exercise of $>1.10$.

Absolute values at peak exercise $\left(\mathrm{VO}_{2 \text { peak }}\right)$ will be calculated as the average value over the last 30 seconds prior to termination of the test. Peak heart rate is defined as the highest heart rate achieved during the cardiopulmonary exercise test. The ventilatory anaerobic threshold (VAT) will be defined as the point at which the ventilatory equivalent for oxygen and the partial end-tidal oxygen tension reached a minimum and thereafter begins to rise in a consistent manner, coinciding with an unchanged ventilatory equivalent for carbon dioxide and partial end-tidal carbon dioxide tension [26]. The VAT will subsequently be expressed as an absolute and relative value (normalized for body mass). The oxygen uptake efficiency slope (OUES) will be calculated using the following equation: oxygen uptake $=a \times \log$ (minute ventilation) $+b$, in which the constant ' $a$ ' represents the rate of increase in oxygen uptake in response to an increase in minute ventilation, called the oxygen uptake efficiency slope (regression coefficient) and ' $b$ ' corresponds to the intercept [27]. A steeper slope, reflected by a higher oxygen uptake efficiency slope, represents a more efficient oxygen uptake: a smaller ventilation quantity is required for a given oxygen uptake. Oxygen uptake efficiency slope values will also be normalized for body mass (OUES/kg) [28]. $\mathrm{VO}_{2 \text { peak, }}$ VAT, and the OUES are objective and valid measures of aerobic capacity in elderly patients scheduled for major abdominal surgery [28-31].

\section{Nutritional status}

To get an insight into the patients' nutritional status the short nutritional assessment questionnaire (SNAQ) will be filled out prior to the start of the supervised home-based prehabilitation program. The SNAQ is a 4-item screening tool that classifies patients as well nourished ( $<2$ points), moderately malnourished ( $\geq 2$ but $<3$ ), and severely malnourished $(\geq 3)$ [32]. Based on the total score, a treatment plan is developed that consists of energy- and protein-enriched meals, and 2 in-between meal snacks for moderately malnourished patients, and treatment by a dietitian along with energy- and protein-enriched meals for severely malnourished patients [33]. Tube feeding and parenteral nutrition will be considered as a potential intervention, if deemed appropriate by the dietitian. The SNAQ is a quick, easy-to-use validated and reproducible tool, which can be used to identify complex, malnourished patients $[32,33]$. 


\section{Health-related quality of life}

The European organization for research and treatment of cancer quality of life questionnaire (EORTC QLQ-C30) is a questionnaire with 30 items developed to assess the perceived quality of life in patients with cancer or cancer survivors. The questionnaire contains several domains of perceptions of quality of life, physical functioning, social functioning, emotional functioning, and cognitive functioning. The questionnaire has a good test-retest reliability (Pearson correlation of 0.79-0.91) [34]. A recent study suggests that scores changing $\geq 10$ points on the EORTC QLQ-C30 should be highlighted for clinical attention [35]. This questionnaire will be filled out before the start of the prehabilitation program and after finishing the program.

\section{Health status}

Perceived health status will be measured by using the EuroQol Five-dimensional questionnaire (EQ-5D). This questionnaire has 14 items in five domains (mobility, selfcare, usual activities, pain, and anxiety/depression). Each domain has to be scored by respondents according to three levels (no problems, some problems, extreme problems), in which patients are asked to give their health state as of today [36]. The total score indicates the perceived health status of the patient. A higher score indicates a lower perceived health status. The EQ-5D has an acceptable validity and responsiveness [36] and percentages of agreement in test-retest reliability range between 69.8 and $99.7 \%$ [37]. Patients will be asked to fill out this questionnaire before and after the home-based prehabilitation program.

\section{The supervised home-based prehabilitation program}

As part of usual care, patients will start with the personalized home-based prehabilitation program under (semi-) supervision of a specifically trained outpatient physical therapist (at home, usual care). For approximately four weeks, the patients will be visited by the physical therapist for functional exercise training. There will be two supervised sessions and one unsupervised training session each week. The program is a combination of functional exercise training with a moderate to high exercise intensity aiming to improve aerobic capacity and muscle strength $[15,17,22,23]$. During the prehabilitation program, the physical therapist will monitor and evaluate physical fitness and physical functioning (e.g., muscle strength, functional mobility) with non-sophisticated performance-based tests (e.g., 2-minute walk test, 30-second chair stand test, stair-climb test) to evaluate and optimize the program (usual care) $[17,23]$.

\section{Physical activity diary}

Patients participating in the study will be asked to fill out a physical activity diary (Figure 2) to get insight in their physical activity level (in minutes per day). The diary is developed for this study and contains different activities of daily living, in which patients have to 
report the number of minutes of a specific activity on the day(s) that the activity is performed. During the supervised home-based training sessions, the physical therapists will verify whether the participant filled out the physical activity diary.

Table 1. Physical activity diary.

\begin{tabular}{|c|c|c|c|c|c|c|c|}
\hline \multirow[t]{2}{*}{ Week prehabilitation: $1 / 2 / 3 / 4$} & \multicolumn{7}{|c|}{ Amount of minutes a day } \\
\hline & Mon & Tue & Wed & Thu & Fri & Sat & Sun \\
\hline $\begin{array}{l}\text { Heavy domestic work (e.g. } \\
\text { vacuuming, cleaning the floor) }\end{array}$ & & & & & & & \\
\hline $\begin{array}{l}\text { Moderate domestic work (e.g., } \\
\text { dusting, doing the dishes) }\end{array}$ & & & & & & & \\
\hline Shopping for groceries & & & & & & & \\
\hline $\begin{array}{l}\text { Activities in and around the house } \\
\text { (e.g., gardening, doing chores). }\end{array}$ & & & & & & & \\
\hline $\begin{array}{l}\text { Family-related activities (e.g., taking } \\
\text { care of (grand)children) }\end{array}$ & & & & & & & \\
\hline Walking & & & & & & & \\
\hline Cycling & & & & & & & \\
\hline $\begin{array}{l}\text { Doing exercises (without supervision } \\
\text { of the physical therapist) }\end{array}$ & & & & & & & \\
\hline $\begin{array}{l}\text { Recreational activities (e.g., reading, } \\
\text { playing cards). }\end{array}$ & & & & & & & \\
\hline
\end{tabular}

\section{Post-prehabilitation measurements}

After the four-week prehabilitation program, participants will revisit the hospital to undergo the post-prehabilitation tests and questionnaires, consisting of a venipuncture to reassess immune system functioning prior to the post-prehabilitation CPET, a postprehabilitation CPET to reassess aerobic capacity $\left(\mathrm{VO}_{2 \text { peak, }} \mathrm{VAT}\right.$, and OUES), the SNAQ to reassess nutritional status, the EORTC QLQ-C30, to reassess health-related quality of life, and the EQ-5D to reassess health status. Patient appreciation of the supervised homebased prehabilitation program will be measured after the program with an appreciation questionnaire. The questionnaire consists of six statements that should be answered on a 5 -point Likert scale ( 1 =totally disagree, $5=$ totally agree). The questionnaire contains the following statements; The goal of the prehabilitation program was clear to me; during the screening at the hospital, I experienced a lot of fatigue; during the training sessions (at home), I experienced a lot of fatigue; I think the screening at the hospital was useful; I think the home-based prehabilitation program was useful; I think the physical therapy treatments were a good preparation (for the scheduled surgery). The higher the total 
score, the higher the appreciation of the prehabilitation program. The questionnaire is based on previous research of Dronkers et al. [38,39]. The abovementioned postprehabilitation measurements will take place at the last study visit, which will be scheduled on a day that the patient already visits the hospital. After this last study visit, the patients will undergo surgery as usual. Postoperatively, patients will receive treatment as usual.

\section{Postoperative clinical outcomes}

Patient characteristics, surgical procedure and data on postoperative outcomes will be obtained from the electronic patient files. Postoperative outcomes consist of time to recovery of physical functioning, postoperative complications (within 30 days after surgery), mortality (within 30 days after surgery), length of hospital stay, length of intensive care admission and 30-day readmissions. Time to recovery of physical functioning will be measured using a modified version of the Iowa level of assistance scale (mILAS) [40]. This modified version assesses the capability of patients to perform several activities of daily living (transfer from supine position to sitting position and vice versa, transfer from sit to stand, walking, and stair climbing) and rates the amount of assistance needed performing these activities. Time to recovery of physical functioning will be defined as the time between the day of surgery and the day at which patients reach a mILAS score of 0 (in days). Postoperative complications will be registered with the use of the Clavien-Dindo classification [41]. 

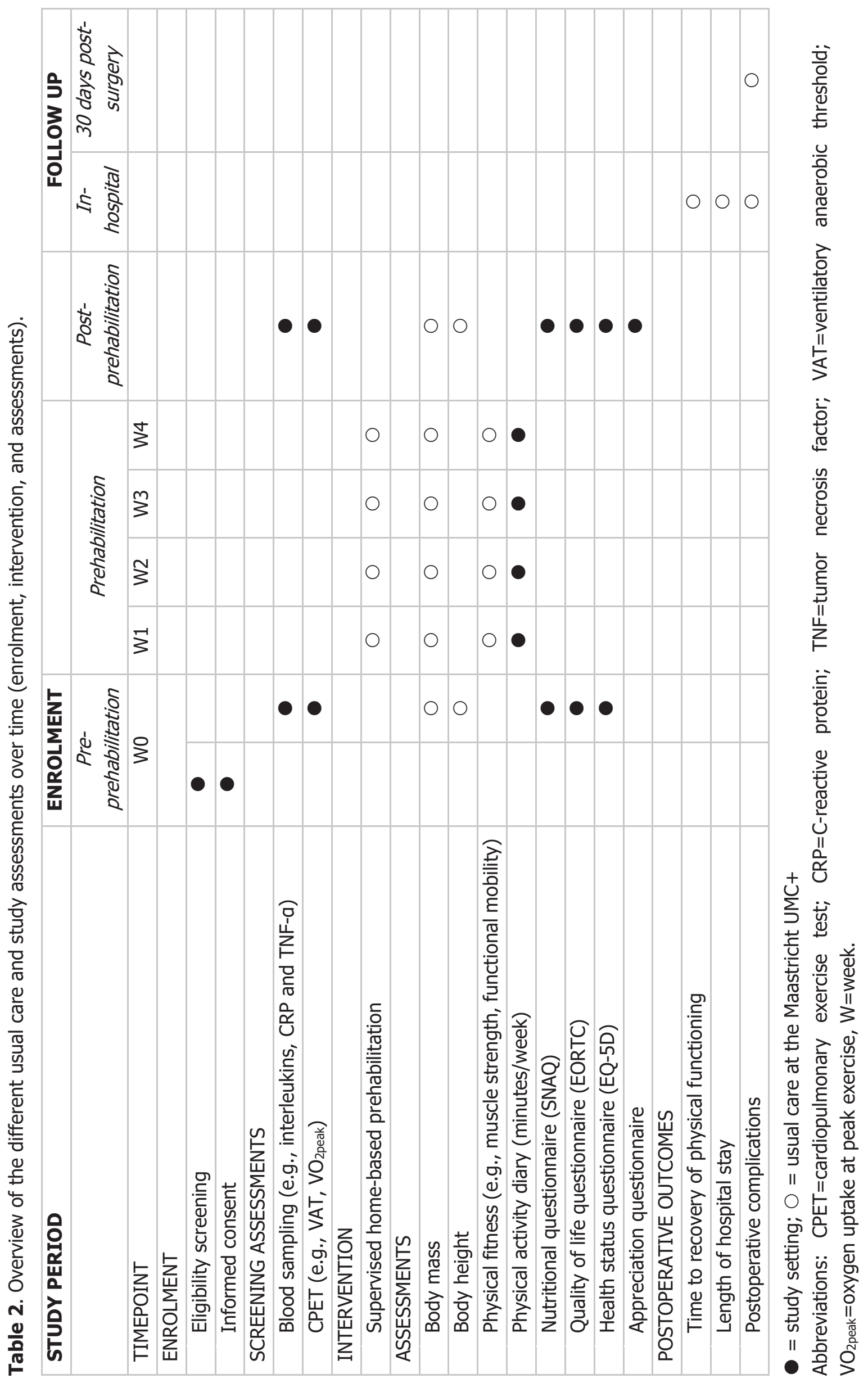


\section{Study outcomes}

\section{Primary study parameter}

The main study parameter is the feasibility (adherence/compliance, adverse events, and patient appreciation) of the supervised home-based prehabilitation program.

\section{Secondary study parameters}

Secondary, the change in physical fitness over time (pre- and post-prehabilitation aerobic capacity, muscle strength, and functional mobility during the prehabilitation program) in individual high-risk patients scheduled for elective colorectal, hepatic, or pancreatic resection enrolled in a four-week supervised and personalized home-based prehabilitation program (which is usual care at our hospital) will be studied. Other secondary outcome measures are immune system functioning, nutritional status, quality of life, and health status before and after the prehabilitation program. Shortterm postoperative outcomes (e.g., time to recovery of physical functioning, 30-day morbidity) will also be collected for descriptive purposes.

\section{Other study parameters}

Changes in physical fitness and postoperative outcomes can also be influenced by the following variables, which will be collected as well. Age, sex, body mass index, comorbidities (Charlson comorbidity index), surgical procedure (open resection or laparoscopic resection), (neo)adjuvant therapy (yes/no), and American Society of Anesthesiologists (ASA) score will be collected from the electronic patient files.

\section{Safety}

All adverse events reported spontaneously by the participant or observed by the supervising physical therapist, investigator, or his staff will be recorded.

\section{Data analysis}

The Statistical Package for the Social Sciences for Windows (version 23.0; IBM, SPSS Inc., Chicago, IL, USA) will be used for statistical analysis. Descriptive statistics will be used to explore the data and the date will be presented as median and interquartile range (IQR) or mean and standard deviation (SD). Categorical data will be summarized by frequency and percentage. Shapiro-Wilk tests for normality will be performed in order to evaluate the data distribution of each parameter. To answer the primary aim, feasibility of the program, adherence/compliance to the program, adverse events, and patient appreciation will be described, by the use of descriptive statistics. The data will be presented as median and IQR or mean and SD, as appropriate. Secondly, to evaluate individual responses over time, individual response 
profiles will be constructed for physical fitness measurements (e.g., muscle strength, functional mobility) throughout the prehabilitation program. Furthermore, to evaluate individual responses to prehabilitation in terms of aerobic capacity, immune system functioning, quality of life, health status, and nutritional status, individual pre-post prehabilitation changes will be graphically depicted. The paired samples t-test or Wilcoxon signed rank test will be performed, as appropriately, to test for statistically significant pre-post differences on these measures. Bonferroni correction for multiple testing will be applied. A P-value $<0.05$ will be considered statistically significant.

\section{DISCUSSION}

Morbidity rates after resection of colorectal, hepatic, and pancreatic tumors are high $[3,4]$. Frail patients often have less ability to cope with surgical stress and hospitalization, which makes them more prone to postoperative complications. These patients might benefit from tailored preoperative interventions (prehabilitation). Assessment of aerobic capacity, as indicated by the VAT assessed during a CPET, can be used to identify high-risk patients (VAT $\leq 11 \mathrm{~mL} / \mathrm{kg} / \mathrm{min}$ ). Prehabilitation can improve the physical fitness of these high-risk patients before major abdominal surgery [15-22]. Currently, there is a lack of information in the literature on the feasibility of and individual responses to a supervised home-based prehabilitation program. The primary objective of the current pilot study is to evaluate the feasibility (adherence/compliance, adverse events, and patient appreciation) of a four-week home-based personalized prehabilitation program in high-risk patients scheduled for elective colorectal, hepatic, or pancreatic resection. Secondary objectives are to evaluate individual responses to prehabilitation in terms of physical fitness (e.g., muscle strength, functional mobility) and to assess within-subject immune system responses, nutritional status, individual self-reported quality of life, and individual self-reported health status before and after the prehabilitation program. Concerning the primary objective, we hypothesize that home-based personalized prehabilitation is safe with a high program adherence/compliance (adherence rate/compliance $>80 \%$, no adverse events) and high patient appreciation (a sum score on the appreciation questionnaire of $>20$ ). Concerning the secondary objectives, we expect that the home-based prehabilitation program will increase physical fitness, selfreported quality of life, and health status in individual patients, whereas no change in immune responses and nutritional status is expected to occur.

The present study has strengths and limitations. The strengths of this study are the selection of high-risk patients, evaluating a home-based prehabilitation program with supervised training sessions, in order to increase inclusion and adherence/compliance rates compared to hospital-based prehabilitation programs or home-based programs 
without supervised sessions. Furthermore, the use of validated measurement instruments is a strength of this study. Limitations are the small sample size and the lack of randomization and a control group. Therefore, a cause-effect relationship cannot be established. Furthermore, patient inclusion could still be a challenge, due to possible personal, logistical and time limitations (e.g., restricted time for prehabilitation and additional measurements before surgery).

This protocol is the first step towards the further optimization of the currently used four-week supervised and personalized home-based prehabilitation program (usual care) of our hospital, by specifically evaluating the feasibility of this program. Hence, the study has a clear explorative character that will look at within-subject responses to the program as well. Results of this exploratory study can subsequently be used to further optimize the hospital's prehabilitation program and will also provide information on how to proceed in subsequent effect studies with sufficient sample sizes.

\section{DECLARATIONS}

\section{Ethics approval and consent to participate}

The study will be conducted according to the principles of the Declaration of Helsinki (WMA Declaration of Helsinki - Ethical Principles for Medical Research Involving Human Subjects, 64th WMA General Assembly, October 2013), the principles of Good Clinical Practice (GCP) and in accordance with the Medical Research Involving Human Subjects Act (WMO). 


\section{REFERENCES}

1. The Netherlands Comprehensive Cancer Organisation (IKNL), Cijfers over kanker, NL 2018. https://www.cijfersoverkanker.nl/selecties/Dataset_1/img5d09faff4c555, 2019 (accessed February 2019).

2. Dutch Institute for Clinical Auditing (DICA) Leiden, DICA Jaarrapportage 2016: DSCA 2016. https://dica.nl/jaarrapportage-2016/dsca, 2019 (accessed February 2019).

3. Andreoni B, Chiappa A, Bertani E, et al., Surgical outcomes for colon and rectal cancer over a decade: results from a consecutive monocentric experience in 902 unselected patients, World J Surg Oncol. 5 (2007) 73.

4. Burns EM, Bottle A, Aylin P, et al., Variation in reoperation after colorectal surgery in England as an indicator of surgical performance: retrospective analysis of Hospital Episode Statistics, $\mathrm{Br}$ Med J. 343 (2011) d4836.

5. Older P, Hall A, Clinical review: how to identify high-risk surgical patients, Crit Care. 8 (2004) 369-372.

6. Older $\mathrm{P}$, Anaerobic threshold, is it a magic number to determine fitness for surgery? Perioper Med (Lond). 2 (2013) 2.

7. Levett $\mathrm{DZ}$, Grocott MP, Cardiopulmonary exercise testing for risk prediction in major abdominal surgery, Anesthesiol Clin. 33 (2015) 1-16.

8. Snowden $\mathrm{CP}$, Prentis J, Jacques B, et al., Cardiorespiratory fitness predicts mortality and hospital length of stay after major elective surgery in older people, Ann Surg. 257 (2013) 999-1004.

9. West MA, Parry MG, Lythgoe $D$, et al., Cardiopulmonary exercise testing for the prediction of morbidity risk after rectal cancer surgery, Br J Surg. 101 (2014) 1166-1172.

10. Hennis PJ, Meale PM, Grocott MP, Cardiopulmonary exercise testing for the evaluation of perioperative risk in non-cardiopulmonary surgery, Postgrad Med J. 87 (2011) 550-557.

11. Nutt CL, Russell JC, Use of the pre-operative shuttle walk test to predict morbidity and mortality after elective major colorectal surgery, Anaesthesia. 67 (2012) 839-849.

12. Struthers R, Erasmus $P$, Holmes $K$, et al., Assessing fitness for surgery: a comparison of questionnaire, incremental shuttle walk, and cardiopulmonary exercise testing in general surgical patients, Br J Anaesth. 101 (2008) 774-780.

13. Reddy S, Contreras CM, Singletary B, et al., Timed stair climbing is the single strongest predictor of perioperative complications in patients undergoing abdominal surgery, J Am Coll Surg. 222 (2016) 559-566.

14. Moran J, Wilson F, Guinan E, et al., The preoperative use of field tests of exercise tolerance to predict postoperative outcome in intra-abdominal surgery: a systematic review, J Clin Anesth. 35 (2016) 446-455.

15. Moorthy K, Wynter-Blyth V, Prehabilitation in perioperative care, Br J Surg. 104 (2017) 802-803.

16. van Rooijen SJ, Engelen MA, Scheede-Bergdahl C, et al., Systematic review of exercise training in colorectal cancer patients during treatment, Scand J Med Sci Sports. 28 (2018) 360-370.

17. Minnella EM, Bousquet-Dion G, Awasthi R, et al., Multimodal prehabilitation improves functional capacity before and after colorectal surgery for cancer: a five-year research experience, Acta Oncol. 56 (2017) 295-300.

18. Carli F, Charlebois $\mathrm{P}$, Stein B, et al., Randomized clinical trial of prehabilitation in colorectal surgery, Br J Surg. 97 (2010) 1187-1197.

19. West MA, Wischmeyer $\mathrm{PE}$, Grocott MP, Prehabilitation and nutritional support to improve perioperative outcomes, Curr Anesthesiol Rep. 7 (2017) 340-349. 
20. Moran J, Guinan E, McCormick $P$, et al., The ability of prehabilitation to influence postoperative outcome after intra-abdominal operation: A systematic review and meta-analysis, Surgery. 160 (2016) 1189-1201.

21. Bruns ER, van den Heuvel B, Buskens CJ, et al., The effects of physical prehabilitation in elderly patients undergoing colorectal surgery: a systematic review, Colorectal Dis. 18 (2016) 0267-277.

22. Barberan-Garcia A, Ubre M, Roca J, et al., Personalised prehabilitation in high-risk patients undergoing elective major abdominal surgery: a randomized blinded controlled trial, Ann Surg. 267 (2018) 50-56.

23. Tew GA, Ayyash R, Durrand J, et al., Clinical guideline and recommendations on pre-operative exercise training in patients awaiting major non-cardiac surgery, Anaesthesia. 73 (2018) 750768.

24. Bongers $\mathrm{BC}$, Dronkers $\mathrm{J}$, Hulzebos $\mathrm{HJ}$, et al., Optimizing perioperative physical therapy care in major elective surgery to improve surgical outcome in high-risk patients: the Better in, Better out $^{\mathrm{TM}}$ concept, NTVA. 29 (2016) 134-139.

25. Miller MR, Hankinson J, Brusasco V, et al., Standardisation of spirometry, Eur Respir J. 26 (2005) 319-338.

26. American thoracic society; American college of chest physicians, ATS/ACCP Statement on cardiopulmonary exercise testing, Am J Respir Crit Care Med. 167 (2003) 211-277.

27. Baba R, Nagashima $M$, Goto $M$, et al., Oxygen uptake efficiency slope: a new index of cardiorespiratory functional reserve derived from the relation between oxygen uptake and minute ventilation during incremental exercise, J Am Coll Cardiol. 28 (1996) 1567-1572.

28. Bongers $\mathrm{BC}$, Berkel $\mathrm{AE}$, Klaase JM, et al., An evaluation of the validity of the pre-operative oxygen uptake efficiency slope as an indicator of cardiorespiratory fitness in elderly patients scheduled for major colorectal surgery, Anaesthesia. 72 (2017) 1206-1216.

29. Smith, TB, Stonell C, Purkayastha S, et al., Cardiopulmonary exercise testing as a risk assessment method in non cardio-pulmonary surgery: a systematic review, Anaesthesia. 64 (2009) 883-893.

30. Older $P$, Hall $A$, Hader $R$, Cardiopulmonary exercise testing as a screening test for perioperative management of major surgery in the elderly, Chest. 116 (1999) 355-362.

31. Older $P$, Smith R, Courtney $P$, et al., Preoperative evaluation of cardiac failure and ischemia in elderly patients by cardiopulmonary exercise testing, Chest. 104 (1993) 701-704.

32. Anthony PS, Nutrition screening tools for hospitalized patients, Nutr Clin Pract. 23 (2008) 373382.

33. Kruizenga HM, Seidell JC, de Vet $\mathrm{HC}$, et al., Development and validation of a hospital screening tool for malnutrition: the short nutritional assessment questionnaire (SNAQ), Clin Nutr. 24 (2005) 75-82.

34. Hjermstad MJ, Fossa SD, Bjordal K, et al., Test/retest study of the European organization for research and treatment of cancer core quality-of-life questionnaire, J Clin Oncol. 13 (1995) 12491254.

35. Snyder CF, Blackford AL, Sussman J, et al., Identifying changes in scores on the EORTC-QLQ-C30 representing a change in patients' supportive care needs, Qual Life Res. 24 (2015) 1207-1216.

36. Linde $\mathrm{L}$, Sørenson J, Ostergaard $\mathrm{M}$, et al., Health-related quality of life: validity, reliability, and responsiveness of SF-36, EQ-15D, EQ-5D, RAQoL, and HAQ in patients with rheumatoid arthritis, J Rheumatol. 35 (2008) 1528-1537.

37. Ravens-Sieberer U, Wille N, Badia X, et al., Feasibility, reliability, and validity of the EQ-5D-Y: results from a multinational study, Qual Life Res. 19 (2010) 887-897. 
38. Dronkers J, Veldman A, Hoberg E, et al., Prevention of pulmonary complications after upper abdominal surgery by preoperative intensive inspiratory muscle training: a randomized controlled pilot study, Clin Rehabil. 22 (2008) 134-142.

39. Dronkers JJ, Lamberts H, Reutelingsperger IM, et al., Preoperative thearpeutic programme for elderly patients scheduled for elective abdominal oncological surgery: a randomized controlled pilot study, Clin Rehabil. 24 (2010) 614-622.

40. Shields RK, Enloe L, Evans RE, et al., Reliability, validity, and responsivesness of functional tests in patients with total joint replacement, Phys Ther. 75 (1995) 176-179.

41. Dindo D, Demartines N, Clavien PA, Classification of surgical complications: a new proposal with the evaluation in a cohort of 6336 patients and results of a survey, Ann Surg. 240 (2004) 205213. 




\section{Chapter}

General discussion 



\section{MAIN FINDINGS}

This thesis aimed to examine specific elements of physical functioning during the entire people's journey and treatment course of patients with colorectal cancer scheduled for elective abdominal resection. Therefore, the involvement of physical therapists in the clinical pathways concerning these patients in hospitals in the Netherlands was evaluated (chapter 2 ). The results showed a considerable variability in daily practice. Preoperative parameters of physical fitness, treatment characteristics (neoadjuvant therapy, surgical procedures), and postoperative outcomes (time to recovery of physical functioning, incidence and type of postoperative complications, and length of hospital stay) were studied. Results showed an association between preoperative parameters of physical fitness and postoperative outcomes in patients with colorectal cancer scheduled for elective abdominal resection. Moreover, the clinical course of patients with locally advanced rectal cancer receiving neoadjuvant chemoradiotherapy was evaluated. Results showed that aerobic capacity and skeletal muscle mass decreased following neoadjuvant chemoradiotherapy. Additionally, preoperative physical exercise training (prehabilitation) during neoadjuvant treatment was studied and showed that a supervised outpatient physical exercise training program for patients with locally advanced resectable rectal cancer was safe and feasible for the participating patients. Moreover, preparations for the evaluation of a personalized supervised home-based prehabilitation program were made by constructing a study protocol for the evaluation of the feasibility of the program and the monitoring of relevant parameters during the course of the training. Finally, the aim was to develop and implement optimal physical therapy management, during the pre- and postoperative care pathway for patients with colorectal cancer, based upon the study findings. By doing so, personalized health care was supported, in which the appropriate individual physical therapy treatment is executed at the right time, and in the right context. All developments as published in this booklet are meant to support personalized physical therapy that in essence originates from close co-creations between patients and caregivers - here mostly physical therapists.

In this general discussion, the main findings, strengths and limitations of the studies described in this thesis will be reflected and discussed, when relevant in the context of the recent evidence-based literature and, the dynamics of the changing health care system in the Netherlands. Furthermore, practical and methodological considerations, further implications, and recommendations for future care, research and innovation are provided. See Figure 1 for a complete overview of the research journey embedded in the current people's journey. 


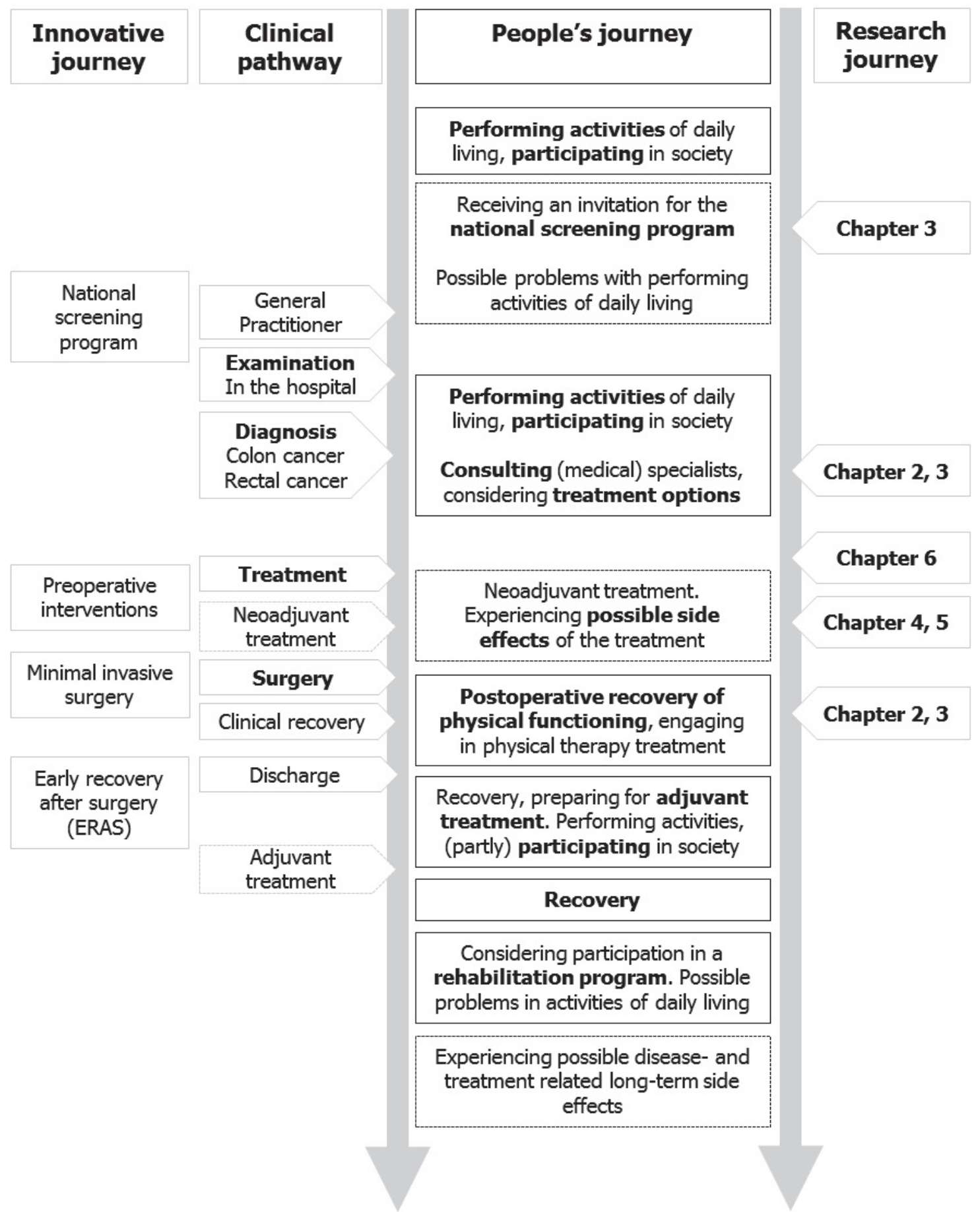

Figure 1. Research journey embedded in the current people's journey. 


\section{Clinical physical therapy practice: revealing differences to reach consensus}

The results in chapter 2 provide an overview of the preoperative and postoperative physical therapy management in Dutch hospitals concerning patients with colorectal, hepatic, or pancreatic cancer scheduled for elective abdominal resection. With this study, differences between daily clinical practice in the Netherlands, and 19 indicators of evidence-based recommendations from the international research literature were evaluated [1-8].

This survey-study focused on the complete care pathway for patients with colorectal, hepatic or pancreatic cancer scheduled for elective abdominal resection. Results from the physical therapy departments of 63 Dutch hospitals (response rate $79 \%$ ) showed some variability in the execution of pre- and postoperative physical therapy. In Figure 2 , the variability between the responding hospitals $(n=63)$ is graphically displayed. The Figure shows the percentage of agreement between the reported physical therapy practice and a set of 19 indicators representing the evidence-based literature (2016-2017). The higher the percentage, the higher the agreement between the reported clinical practice of the concerned hospital and the evidence- and practicebased conceptual models as described in the literature.

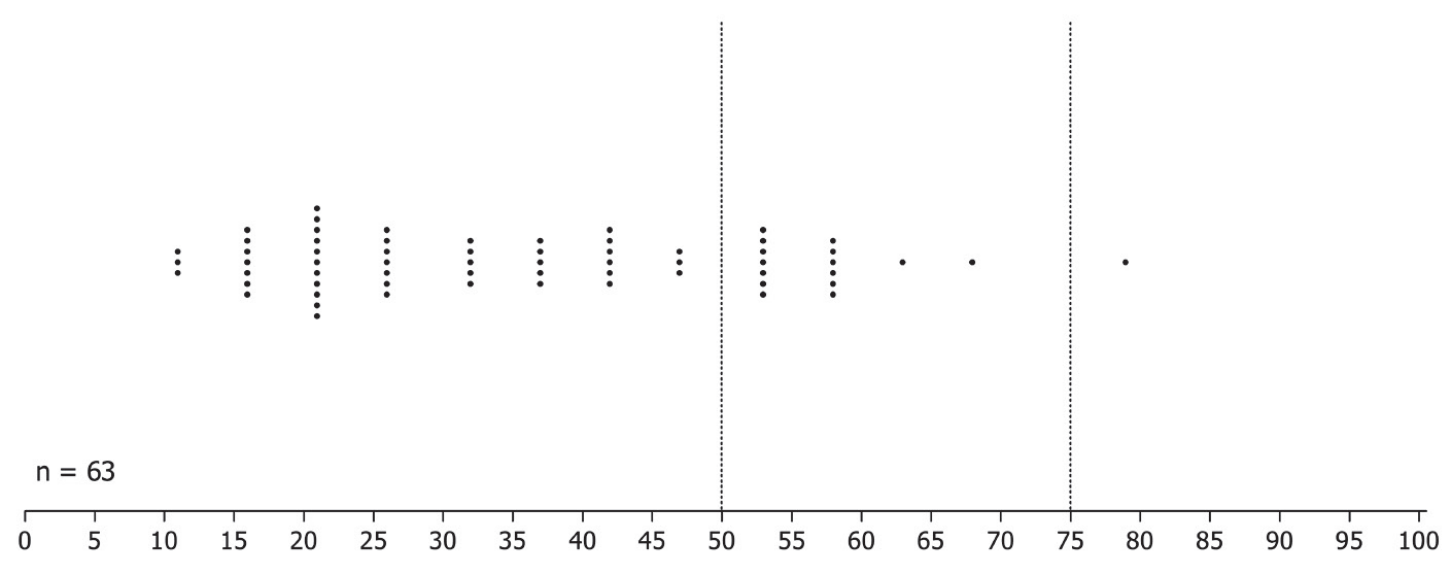

Figure 2. Reported physical therapy practice versus nowadays-advised state-of-the-art physical therapy (\%). The vertical lines indicate $50 \%$ and $75 \%$ agreement between the reported physical therapy management and the evidence-based literature. 
Only one hospital already reported developments in their current clinical practice related to evidence-based literature that can further improve pre- and postoperative physical therapy care for individual patients (see Figure 2). A group of 16 hospitals reported a correspondence of more than $50 \%$ with the indicator-set. Especially, the involvement of the physical therapist in the preoperative phase and the availability of preoperative interventions to prepare high-risk patients properly for the scheduled surgery were reported by these 16 hospitals.

Results of this study can be used to start a (national) debate on optimal pre- and postoperative physical therapy management concerning patients with colorectal, hepatic, or pancreatic cancer scheduled for elective resection. Consensus on core elements of the interventions may help to improve the quality of care and, consequently, induce an improvement in postoperative outcomes. Likewise, a decrease in between-hospital variability may occur, resulting in increased implementation on a nationwide level. Dissensus on the other hand, may help to gain new insights, debate, and most importantly, lead to new questions for research and challenges for innovative evidence-based products and services that may help to improve the physical therapy performance throughout all hospitals. Consequently and based on the indicators, core elements, as preoperative risk assessment and prehabilitation, should be implemented in the clinical care pathways, to ensure optimal, high-quality, evidence-based care for patients with colorectal, hepatic, or pancreatic cancer throughout the Netherlands. The implementation of core elements of pre- and postoperative physical therapy management is a complex process. A strategy in which implementation consists of an interaction between the implementation process, context, and outcomes might be valuable. First, physical therapists should be aware of the available evidence and the related recommendations for clinical practice. Second, in an iterative process, small changes can be made in daily clinical practice with real-time monitoring of relevant outcomes (comparative effectiveness research). This real-time feedback driven adaptations are necessary to make sustainable changes in daily clinical practice $[9,10]$, resulting in the elimination and de-implementation of non-evidence-based physical therapy.

\section{Outcome after resection: measuring postoperative recovery of functioning}

In chapter 3, the strength and statistical significance of associations between preoperative parameters of physical fitness and postoperative outcomes in patients with colorectal cancer scheduled for elective abdominal resection were evaluated. In this explorative observational study, recovery of physical functioning during hospital stay was the main outcome parameter. Recovery of physical functioning was measured in days, using a modified version of the Iowa levels of assistance scale 
(ILAS) [11]. The modified ILAS assesses the capability of patients to perform several activities of daily living (transfer from supine position to sitting position and vice versa, transfer from sit to stand, walking, and stair climbing) and rates the amount of assistance needed by the patient, judged by the physical therapist, when performing these activities. Modified versions of the ILAS are responsive tools to assess the patients' ability to perform daily functional tasks [12], to measure functional change over time during hospital admission [13], and may be useful for outcome assessment in hospitalized patients [14], both on an individual base or in a more aggregated base for the use of benchmark information.

Formerly published studies on postoperative outcomes of hospital stay and surgery often only evaluate the classical outcomes as morbidity and mortality rates [15]. Recovery of physical functioning is a prerequisite of independent functioning in daily life after hospital discharge. Also, hospital-related disability is one of the most significant consequences of admission and surgery, where more than a third of all elderly patients suffer from [16]. Results of our study showed that the choice of the surgical procedure (laparoscopic versus open resection) and the identification of a certain (pre)stadium of colorectal cancer via the Dutch national screening program is related to a 1 - 3 days faster postoperative time to recovery of physical functioning. Furthermore, the preoperative level of physical fitness (walking distance on the incremental shuttle walk test, in meters), functional mobility (timed-up-and-go test, in seconds), and the level of perceived fatigue (multidimensional fatigue index questionnaire) were independent predictors of a faster postoperative recovery of physical functioning. This knowledge enables physical therapists, in co-creation with their patients, to develop and thereupon provide more patient-centered physical therapy care. This care can be based on several indicators that go beyond the more classical risk factors used in clinics and research up to now, in order to facilitate a swift and complete recovery of physical functioning (e.g., by reducing the risk for postoperative morbidity).

Patients with an inadequate level of physical fitness, indicative of a low physiological reserve that is required to cope with the disease and its subsequent treatment, can actively participate in a personalized preoperative training program to improve their preoperative physical potential. Furthermore, patients - especially those at risk, defined by an inadequate level of physical fitness - undergoing more invasive surgical procedures with potential larger impact (e.g., open resection) might need different types of postoperative physical therapy (frequency, duration, content) compared to the more fit patients who will undergo a less invasive surgical procedure (e.g., laparoscopic resection). Previous literature already described preoperative factors, as abnormalities in biomarkers (e.g., hemoglobin and albumin), and inflammatory markers [17], that can negatively predispose postoperative outcomes. Insight in the 
additional factors described in chapter 3 can contribute to a more detailed patient profile, which can pinpoint risks of patients and install proper preventive interventions in time, thereby improving patient-centered care as an intermediate outcome. These outcomes urge for further investigation of the potential added value of physical therapy interventions as proposed here, probably in combination with other interventions formerly already proposed in literature (e.g., nutrition, psychosocial interventions). Chapter 3 shows that there might be room for improvement of both patient's preoperative status as well as the provision of care and care concepts.

\section{Clinical course during neoadjuvant treatment: insight in individual responses}

For rectal cancer, treatment of choice consists of elective abdominal resection, with or without (neo)adjuvant treatment $[18,19]$. In the Netherlands, approximately $60.0 \%$ of the patients with rectal cancer are eligible for neoadjuvant therapy (radiotherapy and/or chemotherapy) prior to elective resection. The dual hit of neoadjuvant treatment and elective resection requests for adequate levels of physical fitness, especially for those that might already be at risk due to their inactive lifestyles and physiological age. Physical fitness (e.g., aerobic capacity, skeletal muscle mass) is an important factor that influences completion of (neo)adjuvant treatment and the incidence of negative side effects during this treatment [20-22]. Physical fitness is also important for a swift and complete recovery of physical functioning after surgery. Furthermore, there is a consistent positive relation between preoperative aerobic capacity and postoperative morbidity and mortality [23-28] and between a low agerelated skeletal muscle mass (defined as sarcopenia) and surgical complications following major abdominal resection [29]. To gain more insight in the clinical course during neoadjuvant treatment in our target population, performance-based physical fitness and computed tomography-derived skeletal muscle measurements before and after neoadjuvant chemoradiotherapy (nCRT) in individual patients with rectal cancer were evaluated (chapter 4).

Our study revealed a statistically significant reduction in physical fitness (aerobic capacity) and skeletal muscle mass following nCRT in the group of patients with locally advanced resectable rectal cancer. Previous studies already found that aerobic capacity was decreased after nCRT as measured by cardiopulmonary exercise testing $[20,23,30]$. There is currently no literature available about the change in physical fitness following nCRT as objectified by other practical performance-based tests (e.g., incremental shuttle walk test, timed-up-and-go test). This makes it difficult to compare our data to other studies. The decrease in skeletal muscle mass is comparable with literature on patients with other gastrointestinal malignancies receiving neoadjuvant chemo(radio)therapy [21,31,32]. More importantly, post hoc 
per patient analysis revealed a rather high amount of inter-individual differences between patients. Some patients even improved slightly during the nCRT-period, whereas almost 50\% really demonstrated worsened fitness at the end of the nCRT. Also, post hoc analysis showed that the patients with a reduced physical fitness after nCRT seem to experience negative side effects during that treatment more often. Previous literature showed that the negative change in skeletal muscle mass seems of more importance than skeletal muscle mass at one single time point and is associated with a poor prognosis/survival [33,34]. Furthermore, sarcopenia is associated with survival, surgical complications, and treatment-related toxicities in patients with colorectal cancer $[22,29]$. Therefore, the recommendation is that all patients should be monitored properly concerning their physical fitness throughout the period of neoadjuvant treatment, to enlighten possible declines in physical fitness and consequently intervene accordingly when this decline occurs. For example, with physical exercise training and/or nutritional support. Insight in the change of physical fitness, body mass, and (adequate) physical activity can simulate patients to opt for additional interventions during the pre- and postoperative treatment course and follow tailored advise to stabilize or to improve their physical fitness and patientspecific outcomes (e.g., patient satisfaction, perceived quality of life).

\section{Prehabilitation during neoadjuvant treatment: safe and feasible}

Neoadjuvant chemoradiotherapy (nCRT) before rectal cancer surgery can lead to a reduction in physical fitness of patients, and physical exercise interventions following this treatment can help them to return physical fitness back to baseline levels $[21,30,35]$. However, are patients able to already start with physical exercise training interventions during neoadjuvant treatment? In chapter 5 , the feasibility and preliminary effectiveness of a supervised outpatient physical exercise training program during neoadjuvant chemoradiotherapy in patients with rectal cancer was evaluated. To the best of our knowledge, our study was one of the first that evaluated physical exercise training in patients with rectal cancer during neoadjuvant treatment. The results showed that the physical exercise program was safe and feasible for the participating patients. However, it appeared to be difficult to persuade and include all eligible patients in the exercise training program, as only a part of patients with rectal cancer was willing and able to participate. The 20 patients that visited our hospital and received the diagnosis rectal cancer with the options nCRT and surgery in sight, all were invited to participate in the training program, of which seven (35.0\%) were not able or not willing to participate and during the program four of 13 patients (30.8\%) dropped out. These figures demonstrate that overall, only nine patients completed the program and evaluations (with an attendance rate of $96 \%$ of the supervised outpatient clinic training sessions). These nine patients were able to 
complete the program with a progressive increase in training load, without any adverse events.

Relevant outcomes of our evaluation were the following: Patients had to visit the hospital for the training sessions. Inclusion was lacking some probably highly important psycho-social ingredients that may help the patient to actively and positively cope with this major life event, the diagnosis and possible interventions upfront. During the first 5.5 weeks, training sessions were combined with the radiotherapy sessions in our hospital which was well-appreciated by the patients. For the remaining preoperative period, patients had to visit the hospital for the training sessions alone, which might have been an overall burden to participate. The participation rate might have been higher, and the dropout rate might have been lower, if the physical exercise program was delivered in the individual patient's own living situation, in a community-based or home-based setting. Previous literature in orthopedic surgery showed that preoperative supervised home-based physical therapy is feasible and has a good inclusion rate of $70 \%$ [36], whereas the sort of training with these kind of patients in the outpatient clinic of the hospital demonstrated an inclusion rate of only 34\% [37]. Previous studies that also evaluated a prehabilitation program for patients with rectal cancer during nCRT showed that prehabilitation is feasible safe, that it may enhance physical fitness, and that the risk of postoperative complications can be modified $[35,38,39]$. However, in all of these studies, inclusion rates were low (between $56.3 \%$ and $62.0 \%$ ) and a large part of the eligible patients did not participate $[35,38,39]$. An important next step according to our reflections consequently is to improve participation rates of patients in physical exercise prehabilitation programs [40], and to evaluate the methodological approach of these feasibility studies.

Previous studies actually have shown that study populations are often not representative for the patient population generally seen in clinical practice. Older adults with multimorbidities are often excluded from the studies, which impairs the generalizability of the study results $[41,42]$. Due to the advanced health care system, the age of people living with (the consequences of) cancer is increasing [43]. Moreover, besides the cancer diagnosis, comorbidities are more frequent nowadays in an overall aging patient population [43]. Therefore, study interventions really need to include a representative sample size and population from the entire patient population of interest, in this case also or even specifically the high-risk patients, to gain generalizable results. In our pilot study, the presence of contraindications for performing physical exercise training was the only reason for exclusion. However, the possibility remains that more fit and enthusiastic patients participated in this study, which could have led to selection bias. Furthermore, in this unimodal proof of concept study, physical exercise training was the only intervention. In this study, there was 
no evaluation of physical exercise training in combination with other interventions, such as nutrition. However, the nutritional status of the patient was evaluated during the treatment course (usual care). In the case of malnutrition or undernutrition, nutritional supplements were provided. Several studies already investigated the effect of preoperative exercise training in combination with nutritional optimization in major elective surgery [44-46]. In most studies, the potentially added value of these preoperative interventions are supported. Moreover, preoperative nutritional support is becoming more important as an in general seen essential component of preoperative surgical care. However, currently available studies are too heterogeneous to conclude that preoperative nutritional support enhances the preoperative condition of - especially high-risk - patients undergoing elective surgery [47-49].

\section{Home-based prehabilitation: personalized program for high-risk patients}

Chapter 6 includes a study protocol for the evaluation of a supervised home-based prehabilitation program for high-risk patients undergoing major elective abdominal resection. All of the former critics discussed in this chapter provided new insights for the appropriate methodology of such a study. Prehabilitation programs have been shown to improve physical fitness before surgery in patients undergoing major abdominal surgery; however, results about postoperative benefits are either positive $[50,51]$ or inconclusive $[52,53]$. A recent study showed that prehabilitation can improve physical fitness when performed in high-risk patients before major abdominal resection, as well as its beneficial effects on postoperative morbidity and length of hospital stay in these patients [50]. A study of Huges et al. showed that prehabilitation can reduce overall and pulmonary morbidity after major abdominal surgery [51]. Literature shows that the content and execution of prehabilitation programs is heterogeneous and the selection of in- and exclusion criteria is diverse $[24,29,52]$. Furthermore, evidence regarding the feasibility and individual responses to a supervised home-based prehabilitation program in high-risk patients undergoing major abdominal surgery is lacking. Therefore, home-based supervised prehabilitation has our specific interest for a future (pilot or feasibility study) evaluation. Results of this exploratory study can subsequently be used to further optimize the prehabilitation program, and to promote a sustainable, cost-effective implementation. Likewise, this study should help to improve research, both feasibility studies and hopefully also the full-blown epidemiological approaches. Figure 3 provides the results of the embedded research journey at the Maastricht UMC+ that was executed during the past 4 years. 


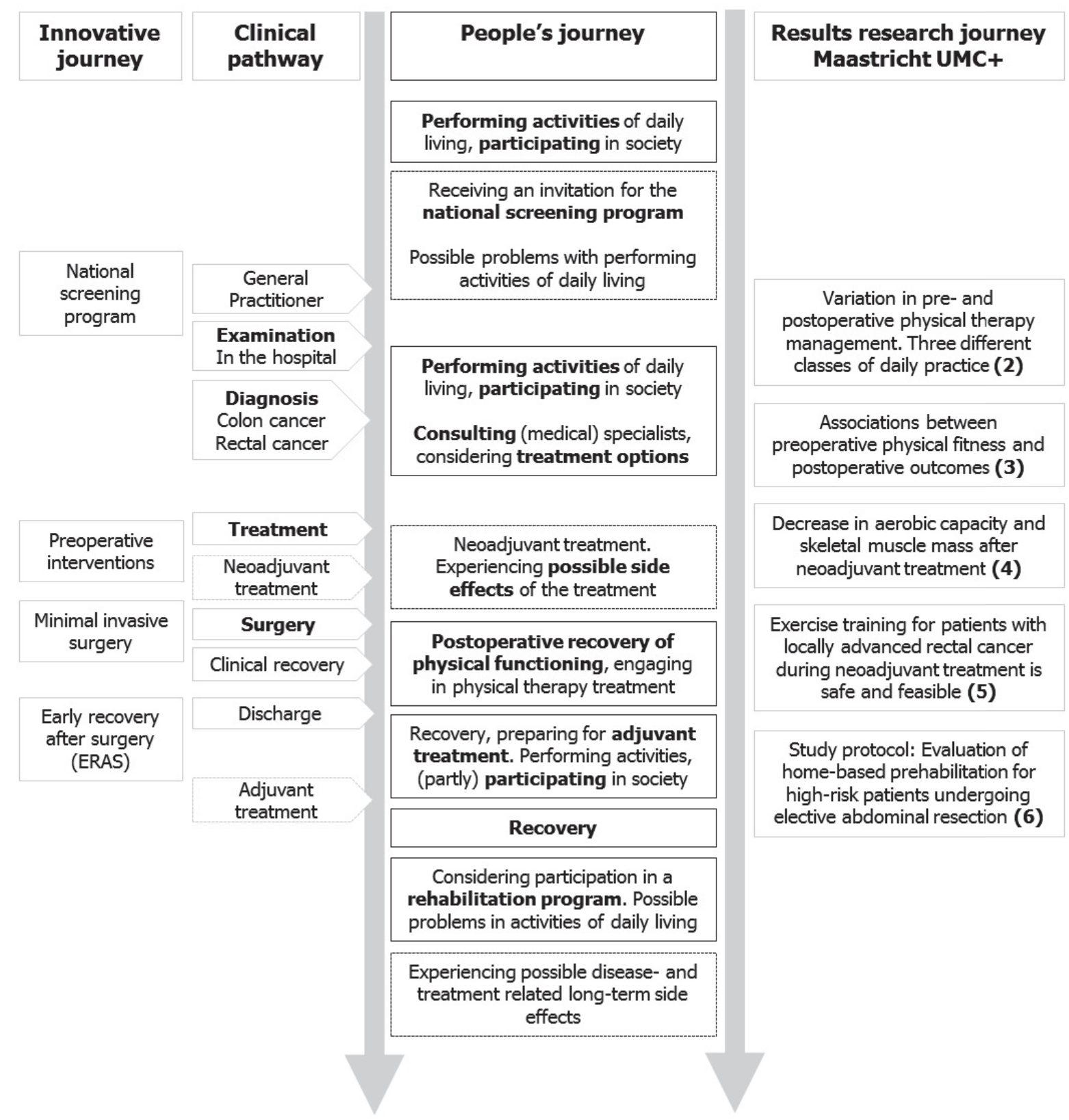

Figure 3. Results of the research journey at the Maastricht UMC+ from 2015 to 2019. 


\section{PRACTICAL AND METHODOLOGICAL CONSIDERATIONS}

One of the strengths of the studies described in this thesis is that the work was coordinated and completed by so called "embedded scientists". Usual daily clinical practice was combined with gathering relevant usual care data to enable the evaluation of the effectiveness of the delivered care, as well as to assess the care innovations aiming to optimize health care. Efforts embedded and directly stemming from the real life practice in the clinic environment of an academic university hospital that has, because of its both urban and rural environment and assignment, a clinical research mission as well as a peripheral hospital mission combined.

Embedded scientists continuously gather new insights and knowledge by collecting, analyzing, and interpreting data from patients during daily clinical routines. This increases the opportunities to translate study results more directly into daily clinical practice and the people of interest [54]. In this thesis, (small) groups of individual patients were studied in the real-life context of our hospital with multiple measurement moments (e.g., pre- and postoperative, before and after neoadjuvant treatment). Most results can probably be applied to individual patients and contribute to personalized clinical physical therapy care. Studies with small sample sizes or $n=1$ studies explore variability in an objective way while leading simultaneously to an informed decision about the best way to treat an individual patient using his or her own data. With results from $n=1$ studies, ideally with a large number of observations per patient, the best or optimal intervention for an individual patient can be determined. It contributes to evaluating the effectiveness of interventions among specific patients with different characteristics in their specific context. Moreover, results from multiple $n=1$ studies, gathered in a standardized way, can generate results applicable to a wider population. For example, when $n=1$ studies investigate the same intervention, it is possible to perform combined or meta-analytic studies of the generated data [55]. By combining data from $n=1$ studies, common characteristics among patients who respond best to a specific intervention can be identified, at least that is the conviction that we would want to underline. For example, patients who respond best to a certain intervention may share genotypic, biomarker, clinical, or demographic characteristics [55]. Knowledge of these common aspects helps health care professionals by choosing a particular intervention for future patients with comparable characteristics. For the profession of physical therapy such kind of research efforts may be helpful to enter the approach of personalized physical therapy, in parallel with the shift to personalized health. Future studies have to demonstrate the advantage of this approach, especially in the context of physical therapy as a part of the prehabilitation concept. 


\section{Investigating the people's journey}

The studies published in this thesis, had the aim to explore the complete patient journey of patients with colorectal cancer scheduled for elective resection. The survey study (chapter 2), with a response rate of $79 \%$, gave insight in the variation in current clinical physical therapy practice in the Netherlands and compared these findings to the best available evidence- and practice-based conceptual models in the field of pre- and postoperative physical therapy management concerning major abdominal surgery. The study in chapter 3 provided information on preoperative physical fitness, postoperative outcomes, and the importance of surgical procedures and the national screening program concerning postoperative outcomes [56]. However, it was not possible to predict postoperative outcomes with the tests performed during the preoperative screening, probably due to the small number of patients that suffered from a postoperative complication (lack of statistical power). The results of the study in chapter 4 showed that aerobic capacity and skeletal muscle mass were significantly reduced following neoadjuvant chemoradiotherapy in a substantial part of the patients with locally advanced rectal cancer, with large inter-individual variation concerning alterations in performance-based physical fitness, muscle mass and the experience of negative side effects. The pilot study containing the physical exercise training program (chapter 5 ) showed that only a specific part of the eligible patients were willing and able to participate in an in-hospital physical exercise training program during nCRT. A rather impressive limitation was the aspect of non-participation, probably due al sorts of aspects of the study, form inclusion to the location of the program. Because the period of nCRT can be a physically and mentally demanding period, we specifically chose for an in-hospital supervised physical exercise training program, with the risk of a lower participation and higher dropout rate. However, in the first 5.5 weeks, training sessions were combined with the radiotherapy schedule of the participants, which contributes to patient-centered care. Future programs should focus on the aspects that induce a low participation rate, especially for frail and older people (e.g., the first contact between the patient and the professional, invitation to participate, location, transportation, and training frequency). The physical exercise training program in our pilot study was performed at moderate exercise intensities. There is a possibility that (a part of) the patients could have trained at higher exercise intensities. Training intensity and related progress over time are important factors in the success of a physical exercise training program and per person determined (higher) intensities might have led to even better results. Other studies investigating physical exercise training programs during neoadjuvant treatment for patients with rectal cancer were also performed at a moderate intensity $[35,38,39,57,58]$. Only one study provided insight in an interval training program with moderate and vigorous intensity intervals during nCRT [59]. Previous literature about 
preoperative exercise training in general (without neoadjuvant treatment) shows that individually prescribed supervised high-intensity interval training is effective and safe as exercise therapy prior to surgery [60]. Literature on preoperative high-intensity training programs for patients undergoing neoadjuvant treatment is lacking and needs further research. Additionally, it is still not known from previous studies what the ideal preoperative prehabilitation program in general looks like, concerning the start of it, the exact content, intensity, duration, and frequency [3,61,62]. A recent clinical guideline with recommendations for preoperative exercise training in patients opting for non-cardiac surgery emphasizes the importance of a multimodal program with a combination of aerobic training, resistance training, and inspiratory muscle training. Training frequency, intensity, and duration of each exercise component should be tailored to each individual patient, taking their initial level of physical fitness into account. Furthermore, the exercise program should be supervised [3] and probably the patient and their social support system should be involved, both in the monitoring and evaluation of the progress as well as in the training itself.

In summary, the combination of different studies and embedding research in daily clinical practice contributed to real-life data concerning the patients of interest, and may therefore provide more context-valid outcomes then when investigated in a rather highly controlled research context. Several aspects of the people's journey in detail, with a focus on the patients' potential and (in)abilities, were evaluated. However, research in a usual care setting has its limitations in terms of methodological quality (e.g., statistical power, bias and confounders, lack of a control group). Study results could also be biased by usual care protocols of the concerned hospital or by the people working in it. Furthermore, the workstyle and the willingness to create positive results of embedded scientists can lead to bias. Hence, this research should be further evaluated and refined in a larger context, by combining data from $\mathrm{n}=1$ studies, and the use of proper analysis methods on these data. 


\section{IMPLICATIONS FOR (CLINICAL) PRACTICE}

The findings of this thesis can be used to optimize the care pathway for individual patients with colorectal cancer during the complete treatment course. This is quite a challenge as the results in chapter 2 showed that there has not been one perfect patient care pathway so far, as clinical practice differs significantly between Dutch hospitals. Furthermore, health care and patient populations are continuously changing over time, which requires health care systems that can adapt to these changes (P4 health). The research in this thesis led to several insights, described in the following part, which can be used to guide patients with colorectal cancer more optimal throughout the complete treatment course, despite between-hospital differences concerning the clinical care pathway.

\section{Clinical practice: adaptive hospitals and health care systems}

Based upon our data and data from previous research, the Dutch national screening program has led to changes in the make-up and characteristics of the population of patients with colorectal cancer. Patients identified via this program were younger, more often were men, and had a lower American society of Anesthesiologists (ASA) score compared to patients that were not identified via the screening program $[19,56]$. These changes can influence the patient profile, disease- and treatment related outcomes, postoperative outcomes, and possibly long-term outcomes. Literature shows that surgery in patients identified via the screening program had significantly lower morbidity and mortality rates [56]. This program can discriminate between patients with different disease stages of colorectal cancer and contributes to a more proactive approach, in which the screening is used to make proper treatment decisions. Each patient and each stage of colorectal cancer is different and requires a personalized approach instead of a one-size-fits-all approach in all aspects of the clinical care pathway. Future developments concerning the national screening program may lead to an increase in early stage diagnoses, which requires an inhospital system that reacts and adapts to this increase. For example, more early stage diagnoses may lead to more laparoscopic surgical procedures, which requires changes in current postoperative (nursing) care protocols (e.g., pain management, wound management, and mobilization protocols). Furthermore, a possible increase in the incidence of early stage diagnoses will influence the proposed effect of prehabilitation programs for these patients. For colorectal cancer, the amount of patients who will need extra (preoperative) interventions may decrease, due to advances in the screening program. 


\section{Preoperative risk assessment: the right screening tools}

After confirmation of the diagnosis and the decision about a tailored treatment, patients should have the option to perform an assessment of physical fitness besides the usual medical assessments [4], especially for the frail (mostly) elderly patients $[4,5,63]$. Besides physical fitness, other aspects as nutritional status and psychological status should be assessed as well, in order to optimize postoperative outcomes. Cardiopulmonary exercise testing can be used as a preoperative risk stratification tool, to decide whether a patient's aerobic capacity level is adequate [64-66]. However, this test is relatively expensive, time consuming, and requires trained personal. Ideally, preoperative risk stratification can be done with the help of other performance-based practical field tests (e.g., stair climbing test, step test), which still needs further (external) validation in larger sample sizes [67]. Further costeffectiveness analysis should shed light on the options of choice in the future here, in research as well as in the daily clinical practice.

\section{Preoperative interventions: the right patient, in the right context}

Patients who decide to undergo colorectal resection and who have an inadequate level of physical fitness or meet other criteria that label them as frail and/or high-risk, should be guided through the preoperative phase with extra interventions concerning preoperative optimization (e.g., physical therapy, if indicated combined with nutritional support). When these patients are indicated for preoperative interventions as physical therapy, these are best performed in a community-based or home-based setting. The social support system of the patient (e.g., partner, social relatives) should be involved in the preoperative workup to sufficiently prepare the patient for major surgery. Concerning the intervention itself, this should contain exercises of adequate intensity in order to improve physical fitness. The patient and his social support system should be adequately educated about the importance of an optimal preoperative physical and nutritional status in relation to patient- and surgical-related outcomes. Prehabilitation should really be seen and executed as a team effort. The program itself must be supervised, executed in the right context and should focus on personal risk factors, goals, needs, and preferences to achieve maximal results and maximal therapy adherence [68]. Furthermore, it is important to monitor progression of physical fitness and functional abilities during the preoperative optimization period using performance-based tests in a rather high frequency fashion to assess training progression - and prevent the patient form therapy failure - and to guide physical therapy treatment $[3,69]$. Patients that are opting for neoadjuvant treatment in the preoperative period should be monitored as well, because their physical fitness is more prone to change during the treatment course, as neoadjuvant treatment before surgery can lead to a decrease in physical fitness assessed by cardiopulmonary 
exercise testing, loss of body mass and loss of skeletal muscle mass [20,21,30-32]. Ideally, this monitoring is performed using practical and easy-to-perform tests, which can even be performed by the patients and/or the social support system at home.

In the postoperative phase, physical therapy treatment needs to be adjusted to the patient's (preoperative) abilities, the surgical procedure, and the discharge destination. Test scores from the preoperative screening of physical fitness should be used as reference points to monitor recovery of physical functioning and to guide postoperative physical therapy. Thereby, patients should be stimulated to be physically active during the postoperative phase, to speed up recovery of physical functioning and to prevent catabolic changes in skeletal muscles and bones induced by non-use [70]. Previous research in hospitalized patients above the age of 65 showed that approximately $80 \%$ of a general hospital day is spend in bed [71], and in a large part of the elderly patients the capacity to perform activities of daily living decreases during the hospital stay [16]. The in-hospital culture and infrastructure should spontaneously motivate more on active participation of patients during daily routines when medically and personally possible. For example, wearing their own clothes instead of a hospital pyjama, or having lunch in a living room instead of in bed. Ideally, patients are frequently re-assessed on recovery of their physical fitness and functioning, in order to guide postoperative physical therapy and post-discharge care as home-based physical therapy or rehabilitation programs. This is especially important for patients opting for additional adjuvant treatment after surgery. An adequate level of physical fitness and/or physical activity may positively affect disease- and treatment-related side effects during and after adjuvant chemotherapy $[72,73]$.

\section{P4 health: patient-tailored personalized care}

The overall clinical pathway should be tailored to each individual patient, hereby taking the national screening program, (neo)adjuvant treatment, and the proposed surgical procedure into account. Besides, there should be compliance to already existing accepted care pathways, because of its known benefits. Patients with an adequate preoperative level of physical fitness should receive information about the treatment course, the possible coinciding consequences and how to maintain their functional abilities throughout this treatment course (enhancing self-management), for example with digital applications [74]. For example, games or text messages on a smart phone in order to control risk factors or to optimize adherence to preoperative recommendations [74]. Patients with an inadequate level of physical fitness should therefore receive additional guidance during the treatment course. The latter should focus on predictive, preventive, personalized, and participatory (physical) therapy interventions (P4 health or P4 physical therapy [75]) during the pre- and 
postoperative phase. Moreover, both low- and high-risk patients should be stimulated by health care professionals regarding the importance of physical fitness and physical activity throughout the complete treatment course [3]. The past few years, the amount of literature about prehabilitation for patients with colorectal cancer has been increasing [76-81], which can help to improve the awareness and believes of patients, their relatives, and health care professionals concerning prehabilitation. A recent cross-sectional survey studying the perspectives of anesthetists and surgeons regarding preoperative risk stratification and prehabilitation showed that both specialty groups recognized the link between functional capacity and postoperative outcomes; however, fewer agreed that robust evidence exists for prehabilitation. The majority of anesthetists stated that they do not have an adequate risk stratification before surgery, and most surgeons are open to delay surgery to create preparation time in order to provide the patient and their social support system with the optimal time period to really prepare for surgery [82].

\section{Preoperative interventions: sustainable implementations}

Currently, it is a challenge to fit preoperative interventions for high-risk patients in the clinical care pathways. Concerning the often short time frame between diagnosis and elective resection, the medical staff should create a (window of) opportunity together with the patient and all involved in-hospital disciplines [3,83]. A case manager (often a colorectal care nurse) can play an important role in this process. One thing that can be done, is improving the appointment schedules in the hospital (for example scheduling all the appointments in one week) and consider postponing surgery when possible, to create preparation time. Recent studies have shown that the time from colorectal cancer diagnosis to (curative) resection has no impact on overall survival $[84,85]$. Hence, this allows some preoperative pathway alterations without compromising safety. Enhanced recovery after surgery (ERAS) is an already existing multimodal perioperative care pathway designed to achieve early recovery for patients undergoing major surgery.

In conclusion, with the studies addressed in this thesis, we found several aspects that could improve the classical clinical journey, in order to guide and prepare, especially high-risk, patients more properly through their individual treatment course. There should be the option to perform a preoperative screening of physical fitness, whereafter prehabilitation interventions should be considered for patients at risk for a complicated postoperative period, preferable in the home-context of the patient. Furthermore, postoperative (physical therapy) treatment should be more patient- and procedure-specific. Functional abilities, self-management qualities and surgical procedures should be taken into account in order to facilitate the proper postoperative treatment. The culture and infrastructure of hospitals should focus on (active) 
physical, social, and mental participation, in which patients act in activity performing their normal daily routines as much as possible. Finally, patients should be reassessed on physical fitness just before discharge, in order to guide post-discharge care.

\section{FUTURE RESEARCH}

Results from research can contribute to knowledge extension. Research results are of great value when relevant questions are asked and when relevant outcomes are measured. Furthermore, results should be interpreted in the right context (e.g., time, place, patient population). Hence, more work is needed to make sure that study results reach medical professionals in clinical practice and thereby the patients of interest. Especially with the nowadays increasing health care costs, accurate measurements of health care delivery in the concerned population is necessary. Results from embedded research, in which small populations or single cases are studied, is better applicable to individual patients, rooted in and informed by their socio-cultural context. Hereto, results are more easily incorporated into daily practice and research waste can be reduced. Moreover, the value of research will increase in terms of new knowledge and sustainable changes in clinical practice. Looking at the elaborated Figure concerning the people's journey, several topics can be addressed in future research (Figure 4).

\section{People's journey: preoperative phase}

It is interesting to investigate changes in patient characteristics and treatment modalities since the introduction of the national screening program. Are there procedures or aspects in the clinical journey that became more important, while other aspects became redundant? The possibility that the screening program will contribute to a (binary) discrimination in the total population of patients with colorectal cancer should be further investigated. Future studies concerning prehabilitation should focus on multidisciplinary preoperative risk assessments, to create a complete detailed patient profile including individual risk factors, which can be targeted with personalized preoperative interventions. For example, nutritional or psychological interventions can be added to the physical therapy interventions to offer a multimodal prehabilitation program. Furthermore, high-intensity physical exercise training programs during (neo)adjuvant treatment needs further research.

The health care system and the patients in it, are continuously changing over time. This leads to changes in demographics, treatment options and disease burden. Future research should evaluate risk-stratification and prehabilitation in the light of the continuously changing health care system and patient population. Additionally, studies should focus on the exact content of a prehabilitation program [86], the effect of personalized prehabilitation in high-risk patients, and adequate risk stratification 
tools next to cardiopulmonary exercise testing. Furthermore, it is interesting to consider multimodal prehabilitation options, personalized to the patient's specific needs, which still needs further research [44-48].

\section{People's journey: postoperative phase}

Even fit patients can experience a complicated postoperative course. To evaluate the effects of preoperative optimization on surgery and hospitalization-related outcomes in individual patients, future studies investigating effects on postoperative complications should not only take the incidence of complications into account; however, they should also focus on the impact of a postoperative complication in individual patients. Patients with an adequate level of physical fitness may also have postoperative complications, but the impact may not be as high compared to patients with inadequate levels of physical fitness facing the same complication [87]. There should be several outcome measures developed in which the impact of a complication is also considered. For example, the impact of the complication on the patients' postoperative recovery of physical functioning or the time spend in the hospital should be evaluated. Additionally, several other factors besides physical fitness could influence postoperative outcomes. Future studies should for example look into psychological factors, coping, and anxiety on postoperative outcomes and evaluate the possibilities to perform prehabilitation on these factors $[1,88,89]$. Despite an adequate level of physical fitness, other (personal) characteristics could interfere with postoperative recovery and should not be ignored.

\section{People's journey: general aspects}

Future studies should evaluate longer follow-up periods after discharge (for example 3 months, 6 months, or more) in order to study the possible long-term effects of preand postoperative interventions, which can increase the societal value of research. Additionally, future studies should focus more on patient-specific outcomes, both concerning the preoperative phase (patient appreciation, quality of life during treatment) as well as the postoperative phase (recovery of physical functioning, return to normal activities, quality of life, long-term patient outcomes) instead of clinical outcomes only (e.g., length of stay, postoperative complications, readmissions). Nowadays, the number of people living with the consequences of cancer and the related treatment is increasing, and as a consequence cancersurvivors are more and more seen as people with a chronic disease, which emphasizes the importance of studying long-term outcomes as quality of life and the ability to (re)participate in (social) activities or employment. With studies focusing on these outcomes, the total impact of the disease and treatment on the patient's long-term daily life can be evaluated. 


\section{Core sets in national databases: improving value of collected data}

The development of uniform pre- and postoperative core sets containing functional data can be of great relevance in the future. These core sets should contain measurements to assess and monitor physical functioning of patients with colorectal cancer in Dutch hospitals. First, hospitals should agree on what kind of measurement should be in these core sets (which preoperative measurements and postoperative outcomes). Additionally, the core sets from different Dutch hospitals should be combined in national databases. With cores sets in a large (national) database, usual care data can be evaluated faster and more accurate, making also use of modernistic techniques as machine learning and other sorts of robust artificial intelligence. Moreover, it increases the ability to identify patients who are at risk for postoperative complications prior to colorectal surgery, due to the large amount of data. Furthermore, the (national) core sets (e.g., preoperative physical fitness, postoperative recovery of physical functioning, impact of a complication) can be added to already existing medical databases as the Dutch Colorectal Audit (DCRA) [19], which gathers medically oriented surgical data concerning patients who were scheduled for elective colorectal surgery. This will contribute to the evaluation of perioperative interventions and postoperative outcomes.

By doing all this, the ability to optimally identify patients who are at risk for postoperative complications, mortality, and sometimes permanent loss of physical functioning will increase. These patients might benefit from prehabilitation to prepare them for surgery in order to improve postoperative medical and functional recovery. For society in general, this implies an optimization of health care (perioperative care) and health care costs. Finally, this will lead to an increase in (transparent) information that can guide clinical decision-making. Figure 4 shows the research journey, with additional recommendations for future directions. 


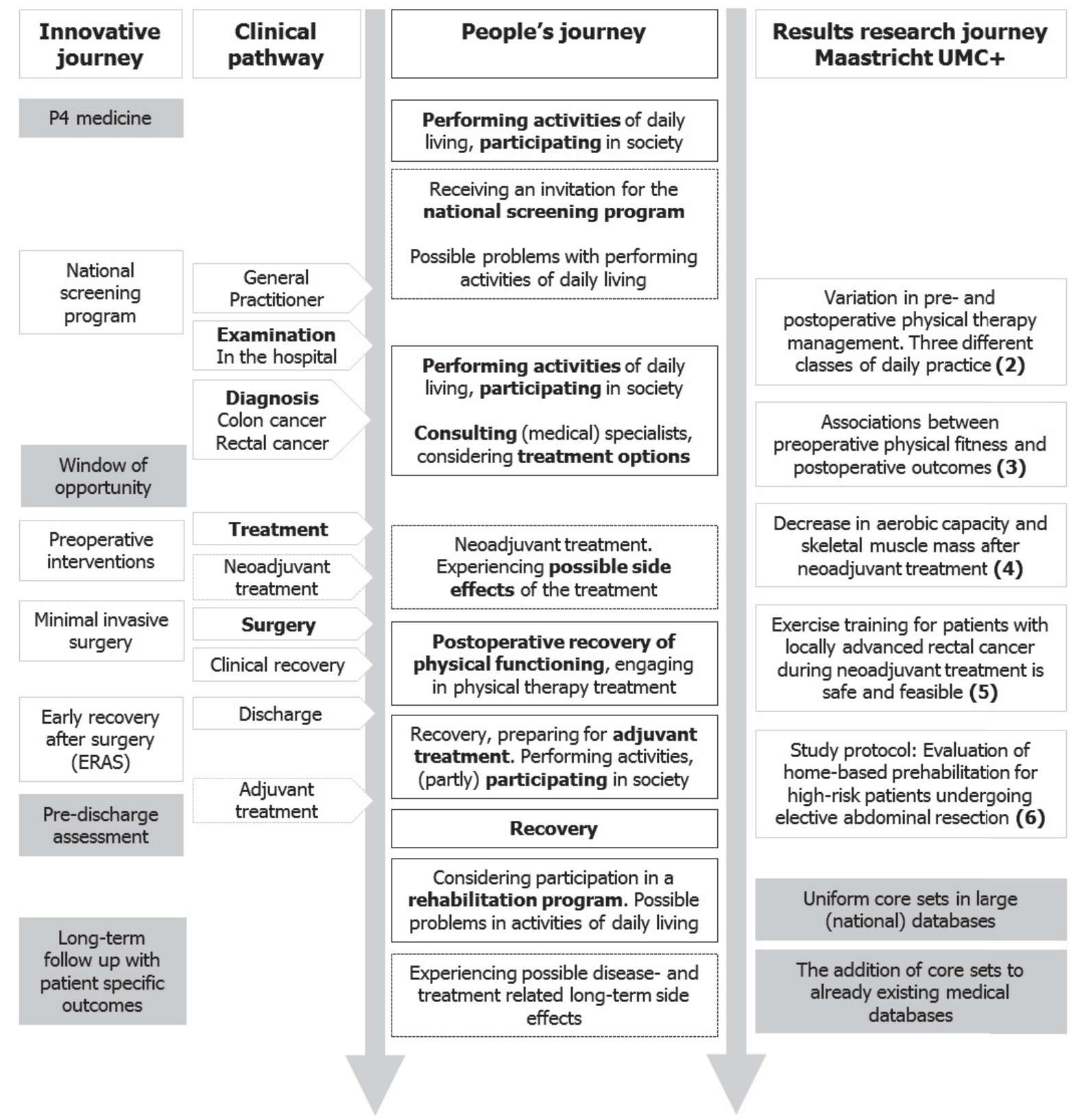

Figure 4. The research journey, with recommendations for future directions. 


\section{FINAL CONCLUSIONS}

The present thesis demonstrated that there is currently a wide degree of variation between hospitals in the Netherlands regarding pre- and postoperative physical therapy practice for patients opting for major abdominal surgery. Furthermore, associations between preoperative physical fitness and postoperative outcomes in patients with colorectal cancer scheduled for abdominal resection were revealed. Future developments concerning the Dutch national screening program, prehabilitation, surgical procedures, and anesthetic procedures require proactive health care systems, which can adapt to these changes. In patients with locally advanced rectal cancer, a reduction in aerobic capacity and skeletal muscle mass following neoadjuvant chemoradiotherapy was found. Moreover, our pilot study demonstrated that a supervised outpatient physical exercise training program for individual patients with locally advanced resectable rectal cancer during neoadjuvant chemoradiotherapy is feasible and safe for the patients that participated.

\section{Lessons learned from the research concerning the colorectal clinical journey}

A preoperative assessment of physical fitness is useful to get a complete overview of the patient profile, which can contribute to a more effective use of resources during the treatment continuum. For patients with inadequate levels of physical fitness or other characteristics that enhance frailty, and who are therefore considered high-risk, pre- and postoperative interventions during the patient journey should be facilitated, probably with help of their (in)formal caregivers and social support system as making it a real team effort. The latter aims to enable them to withstand the (neo)adjuvant, surgical- and hospital-related stress and promote postoperative recovery and clinical outcomes. Furthermore, the structure and infrastructure of the hospital should be focused on (active) participation and performing daily routines.

With the findings in this thesis, there is a better understanding of patients with colorectal cancer in the Maastricht UMC+ (characteristics, disease- and treatmentrelated symptoms), preoperative screening tools, preoperative physical exercise training, and functional recovery after surgery. Hopefully, the embedded research performed during this journey will contribute to a sustainable transition from onesize-fits-all reactive care pathways to proactive, predictive, preventive, personalized, and participatory care pathways. This should be achieved by a multidisciplinary team, which contains all care disciplines involved in the treatment concerning patients with colorectal cancer, to increase the total value of this innovative transition. 


\section{REFERENCES}

1. Wynter-Blyth V, Moorthy K, Prehabilitation: preparing patients for surgery, BMJ. 358 (2017) j3702.

2. Bongers $\mathrm{BC}$, Dronkers $\mathrm{J}$, Hulzebos $\mathrm{HJ}$, et al., Optimizing perioperative physical therapy care in major elective surgery to improve surgical outcome in high-risk patients: the Better in, Better out $^{\text {TM }}$ concept, NTVA. 29 (2016) 134-139.

3. Tew GA, Ayyash R, Durrand J, et al., Clinical guideline and recommendations on pre-operative exercise training in patients awaiting major non-cardiac surgery, Anaesthesia. 73 (2018) 750-768.

4. Hulzebos EH, van Meeteren NL, Making the elderly fit for surgery, Br J Surg. 103 (2016) e12-15.

5. Hulzebos EH, van Meeteren NL, Making the elderly fit for surgery, Br J Surg. 103 (2016) 463.

6. Lassen $\mathrm{K}$, Soop M, Nygren J, et al., Consensus review of optimal perioperative care in colorectal surgery: Enhanced Recovery After Surgery (ERAS) Group recommendations, Arch Surg. 144 (2009) 961-969.

7. Hulzebos EH, Helders PJ, Favie NJ, et al., Preoperative intensive inspiratory muscle training to prevent postoperative pulmonary complications in high-risk patients undergoing CABG surgery: a randomized clinical trial, JAMA. 296 (2006) 1851-1857.

8. Dronkers JJ, Lamberts $\mathrm{H}$, Reutelingsperger IM, et al., Preoperative therapeutic programme for elderly patients scheduled for elective abdominal oncological surgery: a randomized controlled pilot study, Clin Rehabil. 24 (2010) 614-622.

9. Luig T, Asselin J, Sharma AM, et al., Understanding implementation of complex interventions in primary care teams, J Am Board Fam Med. 31 (2018) 431-444.

10. Moore GF, Audrey S, Barker M, et al., Process evaluation of complex interventions: Medical Research Council guidance, BMJ. 350 (2015) h1258.

11. Shields RK, Enloe L, Evans RE, et al., Reliability, validity, and responsivesness of functional tests in patients with total joint replacement, Phys Ther. 75 (1995) 176-179.

12. Elings J, Zoethout S, Ten Klooster PM, et al., Advocacy for use of the modified Iowa Level of Assistance Scale for clinical use in patients after hip replacement: an observational study, Physiotherapy. 105 (2019) 108-113.

13. Kimmel LA, Elliott JE, Sayer JM, et al., Assessing the reliability and validity of a physical therapy functional measurement tool - the modified Iowa level of assistance scale - in acute hospital inpatients, Phys Ther. 96 (2016) 176-182.

14. Soh SE, Stuart L, Raymond M, et al., The validity, reliability, and responsiveness of the modified Iowa Level of Assistance scale in hospitalized older adults in subacute care, Disabil Rehabil. 40 (2018) 2931-2937.

15. Andreoni B, Chiappa A, Bertani E, et al., Surgical outcomes for colon and rectal cancer over a decade: results from a consecutive monocentric experience in 902 unselected patients, World J Surg Oncol. 5 (2007) 73.

16. Covinsky KE, Palmer RM, Fortinsky RH, et al., Loss of independence in activities of daily living in older adults hospitalized with medical illnesses: increased vulnerability with age, J Am Geriatr Soc. 51 (2003) 451-458.

17. Ajitsaria P, Eissa SZ, Kerridge RK, Risk assessment, Curr Anesthesiol Rep. 8 (2018) 1-8.

18. The Netherlands Comprehensive Cancer Organisation (IKNL). Cijfers over kanker, The Netherlands. http://www.cijfersoverkanker.nl/meest-voorkomende -soorten-52.html, 2018 (accessed october 2018).

19. Dutch Institute for Clinical Auditing (DICA) Leiden, DICA Jaarrapportage: DCRA 2017. https://dica.nl/jaarrapportage-2017/dcra, 2018 (accessed October 2018). 


\section{Chapter 7}

20. West MA, Loughney L, Barben $C P$, et al., The effects of neoadjuvant chemoradiotherapy on physical fitness and morbidity in rectal cancer surgery patients, Eur J Surg Oncol. 40 (2014) 14211428.

21. Palmela C, Velho S, Agostinho L, et al., Body composition as a prognostic factor of neoadjuvant chemotherapy toxicity and outcome in patients with locally advanced gastric cancer, J Gastric cancer. 17 (2017) 74-87.

22. van Vledder MG, Levolger S, Ayez N, et al., Body composition and outcome in patients undergoing resection of colorectal liver metastases, Br J Surg. 99 (2012) 550-557.

23. West MA, Loughney L, Lythgoe $D$, et al., The effect of neoadjuvant chemoradiotherapy on wholebody physical fitness and skeletal muscle mitochondrial oxidative phosphorylation in vivo in locally advanced rectal cancer patients-an observational pilot study, PLoS One. 9 (2014) 1-15.

24. Snowden CP, Prentis J, Jacques B, et al., Cardiorespiratory fitness predicts mortality and hospital length of stay after major elective surgery in older people, Ann Surg. 257 (2013) 999-1004.

25. West MA, Lythgoe $D$, Barben $C P$, et al., Cardiopulmonary exercise variables are associated with postoperative morbidity after major colonic surgery: a prospective blinded observational study, $\mathrm{Br}$ J Anaesth. 112 (2014) 665-671.

26. West MA, Parry MG, Lythgoe $D$, et al., Cardiopulmonary exercise testing for the prediction of morbidity risk after rectal cancer surgery, Br J Surg. 101 (2014) 1166-1172.

27. Levett DZ, Grocott MP, Cardiopulmonary exercise testing for risk prediction in major abdominal surgery, Anesthesiol Clin. 33 (2015) 1-16.

28. Hennis PJ, Meale PM, Grocott MP, Cardiopulmonary exercise testing for the evaluation of perioperative risk in non-cardiopulmonary surgery, Postgrad Med J. 87 (2011) 550-557.

29. Boer BC, de Graaff F, Brusse-Keizer M, et al., Skeletal muscle mass and quality as risk factors for postoperative outcome after open colon resection for cancer, Int J Colorectal Dis. 31 (2106) 11171124.

30. West MA, Loughney L, Lythgoe $D$, et al., Effect of prehabilitation on objectively measured physical fitness after neoadjuvant treatment in preoperative rectal cancer patients: a blinded interventional pilot study, $\mathrm{Br}$ J Anaesth. 114 (2015) 244-251.

31. Dalal S, Hui D, Bidaut L, et al., Relationships among body mass index, longitudinal body composition alterations, and survival in patients with locally advanced pancreatic cancer receiving chemoradiation: a pilot study, J Pain Symptom Manage. 44 (2012) 181-191.

32. Daly LE, Ní Bhuachalla ÉB, Power DG, et al., Loss of skeletal muscle during systemic chemotherapy is prognostic of poor survival in patients with foregut cancer, J Cachexia Sarcopenia Muscle. 9 (2018) 315-325.

33. Rutten IJ, van Dijk DP, Kruitwagen RF, et al., Loss of skeletal muscle during neoadjuvant chemotherapy is related to decreased survival in ovarian cancer patients, J Cachexia Sarcopenia Muscle. 7 (2016) 458-466.

34. Liu J, Motoyama S, Sato Y, et al., Decreased skeletal muscle mass after neoadjuvant therapy correlates with poor prognosis in patients with esophageal cancer, Anticancer Res. 36 (2016) 6677-6685.

35. Morielli AR, Usmani N, Boulé NG, et al., A phase I study examining the feasibility and safety of an aerobic exercise intervention in patients with rectal cancer during and after neoadjuvant chemoradiotherapy, Oncol Nurs Forum. 43 (2016) 352-362.

36. Oosting E, Jans MP, Dronkers JJ, et al., Preoperative home-based physical therapy versus usual care to improve functional health of frail older adults scheduled for elective total hip arthroplasty: a pilot randomized controlled trial, Arch Phys Med Rehabil. 93 (2012) 610-616. 
37. Hoogeboom TJ, Dronkers JJ, van den Ende $\mathrm{CH}$, et al., Preoperative therapeutic exercise in frail elderly scheduled for total hip replacement: a randomized pilot trial, Clin Rehabil. 24 (2010) 901910.

38. Singh F, Galvão DA, Newton RU, et al., Feasibility and preliminary efficacy of a 10-week resistance and aerobic exercise intervention during neoadjuvant chemoradiation treatment in rectal cancer patients, Integr Cancer Ther. 17 (2018) 952-959.

39. Moug SJ, Mutrie N, Barry SJ, et al., Prehabilitation is feasible in patients with rectal cancer undergoing neoadjuvant chemoradiotherapy and may minimize physical deterioration: results from The REx trial, Colorectal Dis. 21 (2019) 548-562.

40. Wright EJ, Exercising patient-centredness in prehabilitation programs, Eur J Surg Oncol. 43 (2017) 509-510.

41. Zulman DM, Sussman JB, Chen $X$, et al., Examining the evidence: a systematic review of the inclusion and analysis of older adults in randomized controlled trials, J Gen Intern Med. 26 (2011) 783-790.

42. Van Spall HG, Toren A, Kiss A, et al., Eligibility criteria of randomized controlled trials published in high-impact general medical journals: a systematic sampling review, JAMA. 297 (2009) 12331240.

43. Ikkersheim D, Vernooij K, Wouters A, Toekomstverkenning betere kwaliteit van leven (KWF kankerbestrijding), KPMG Health (2018) 1-93.

44. Gupta R, Gan TJ, Preoperative nutrition and prehabilitation, Anesthesiol Clin. 34 (2016) 143-153.

45. Agrelli TF, Borges MC, Cunha FM, et al., Combination of preoperative pulmonary and nutritional preparation for esophagectomy, Acta Cir Bras. 33 (2018) 67-74.

46. Evans DC, Martindale RG, Kiraly LN, et al., Nutrition optimization prior to surgery, Nutr Clin Pract. 29 (2014) 10-21.

47. van Rooijen S, Carli F, Dalton SO, et al., Preoperative modifiable risk factors in colorectal surgery: an observational cohort study identifying the possible value of prehabilitation, Acta Oncol. 56 (2017) 329-334.

48. Bruns ER, Argillander $T E$, van den Heuvel $B$, et al., Oral nutrition as a form of pre-operative enhancement in patients undergoing surgery for colorectal cancer: a systematic Review, Surg Infect (Larchmt). 19 (2018) 1-10.

49. Gillis $\mathrm{C}$, Wischmeyer PE, Pre-operative nutrition and the elective surgical patient: why, how and what? Anaesthesia. 74 (2019) Suppl 1:27-35.

50. Barberan-Garcia A, Ubre M, Roca J, et al., Personalised prehabilitation in high-risk patients undergoing elective major abdominal surgery: a randomized blinded controlled trial, Ann Surg. 267 (2018) 50-56.

51. Hughes MJ, Hackney RJ, Lamb PJ, et al., Prehabilitation before major abdominal surgery: a systematic review and meta-analysis, World J Surg. 43 (2019) 1661-1668.

52. Moran J, Guinan E, McCormick P, et al., The ability of prehabilitation to influence postoperative outcome after intra-abdominal operation: A systematic review and meta-analysis, Surgery. 160 (2016) 1189-1201.

53. Bruns ER, van den Heuvel B, Buskens $\mathrm{CJ}$, et al., The effects of physical prehabilitation in elderly patients undergoing colorectal surgery: a systematic review, Colorectal Dis. 18 (2016) O2670277.

54. Vindrola-Padros $\mathrm{C}$, Pape T, Utley M, et al., The role of embedded research in quality improvement: a narrative review, BMJ Qual Saf. 26 (2017) 70-80.

55. Lillie EO, Patay B, Diamant J, et al., The n-of-1 clinical trial: the ultimate strategy for individualizing medicine? Per Med. 8 (2011) 161-173. 


\section{Chapter 7}

56. de Neree Tot Babberich MP, Vermeer NC, Wouters MW, et al., Postoperative outcomes of screendetected vs non-screen-detected colorectal cancer in the Netherlands, JAMA Surg. 153 (2018) e183567.

57. Singh F, Newton RU, Baker MK, et al., Feasibility and efficacy of presurgical exercise in survivors of rectal cancer scheduled to receive curative resection, Clin Colorectal Cancer. 16 (2017) 358-365.

58. Brunet J, Burke S, Grocott MP, et al., The effects of exercise on pain, fatigue, insomnia, and health perceptions in patients with operable advanced stage rectal cancer prior to surgery: a pilot trial, BMC Cancer. 17 (2017) 153.

59. Weston M, Weston KL, Prentis JM, et al., High-intensity interval training (HIT) for effective and time-efficient pre-surgical exercise interventions, Perioper Med (Lond). 5 (2016) 2.

60. van Rooijen SJ, Engelen MA, Scheede-Bergdahl C, et al., Systematic review of exercise training in colorectal cancer patients during treatment, Scand J Med Sci Sports. 28 (2018) 360-370.

61. Pouwels S, Stokmans RA, Willigendael EM, et al., Preoperative exercise therapy for elective major abdominal surgery: a systematic review, Int J Surg. 12 (2014) 134-140.

62. Pouwels S, Hageman D, Gommans LN, et al., Preoperative exercise therapy in surgical care: a scoping review, J Clin Anesth. 33 (2016) 476-490.

63. Scheede-Bergdahl C, Minnella EM, Carli F, Multi-modal prehabilitation: addressing the why, when, what, how, who and where next? Anaesthesia. 74 (2019) Suppl 1:20-26.

64. Older PO, Levett DZ, Cardiopulmonary exercise testing and surgery, Ann Am Thorac Soc. 14 (2017) S74-S83.

65. Moran J, Wilson F, Guinan E, et al., The preoperative use of field tests of exercise tolerance to predict postoperative outcome in intra-abdominal surgery: a systematic review, J Clin Anesth. 35 (2016) 446-455.

66. Levett DZ, Jack $S$, Swart $M$, et al., Perioperative cardiopulmonary exercise testing (CPET): consensus clinical guidelines on indications, organization, conduct, and physiological interpretation, $\mathrm{Br}$ J Anaesth. 120 (2018) 484-500.

67. Lee $\mathrm{CH}$, Kong JC, Ismail $\mathrm{H}$, et al., Systematic review and meta-analysis of objective assessment of physical fitness in patients undergoing colorectal cancer surgery, Dis Colon Rectum. 61 (2018) 400-409.

68. Ferreira V, Agnihotram RV, Bergdahl A, et al., Maximizing patient adherence to prehabilitation: what do the patients say? Support Care Cancer. 26 (2018) 2717-2723.

69. Glasziou P, Irwig L, Mant D, Monitoring in chronic disease: a rational approach, BMJ. 330 (2005) 644-648.

70. Bloomfield SA, Changes in musculoskeletal structure and function with prolonged bed rest, Med Sci Sports Exerc. 29 (1997) 197-206.

71. Brown $\mathrm{CJ}$, Redden $\mathrm{DT}$, Flood $\mathrm{KL}$, et al., The underrecognized epidemic of low mobility during hospitalization of older adults, J Am Geriatr Soc. 57 (2009) 1660-1665.

72. Van Vulpen JK, Velthuis MJ, Steins Bisschop CN, et al., Effects of an exercise program in colon cancer patients undergoing chemotherapy, Med Sci Sports Exerc. 48 (2016) 767-775.

73. Furmaniak AC, Menig $M$, Markes $\mathrm{MH}$, Exercise for women receiving adjuvant therapy for breast cancer, Cochrane Database Syst Rev. 9 (2016) CD005001.

74. Michard F, Smartphones and e-tablets in perioperative medicine, Korean J Anesthesiol. 70 (2017) 493-499.

75. Hood $L$, Flores $M, A$ personal view on systems medicine and the emergence of proactive P4 medicine: predictive, preventive, personalized and participatory, N Biotechnol. 29 (2012) 613624. 
76. Piraux E, Caty G, Reychler G, Effects of preoperative combined aerobic and resistance exercise training in cancer patients undergoing tumor resection surgery: A systematic review of randomised trials, Surg Oncol. 27 (2018) 584-594.

77. Gillis C, Fenton TR, Sajobi TT, et al., Trimodal prehabilitation for colorectal surgery attenuates post-surgical losses in lean body mass: a pooled analysis of randomized controlled trials, Clin Nutr. 38 (2018) 1053-1060.

78. Minnella EM, Carli F, Prehabilitation and functional recovery for colorectal cancer patients, Eur ] Surg Oncol. 44 (2018) 919-926.

79. Gillis C, Buhler K, Bresee L, et al., Effects of nutritional prehabilitation, with and without exercise, on outcomes of patients who undergo colorectal surgery: a systematic review and meta-analysis, Gastroenterology. 155 (2018) 391-410.

80. Bruns ER, Rooijen SJ, Argillander TE, et al., Improving outcomes in oncological colorectal surgery by prehabilitation, Am J Phys Med Rehabil. 98 (2019) 231-238.

81. van Rooijen SJ, Engelen MA, Scheede-Bergdahl C, et al., Systematic review of exercise training in colorectal cancer patients during treatment, Scand J Med Sci Sports. 28 (2018) 360-370.

82. Li MH, Bolshinsky V, Ismail $\mathrm{H}$, et al., A cross-sectional survey of Australian anesthetists' and surgeons' perceptions of preoperative risk stratification and prehabilitation, Can J Anaesth. 66 (2019) 388-405.

83. Carli F, Ferreira V, Prehabilitation: a new area of integration between geriatricians, anesthesiologists, and exercise therapists, Aging Clin Exp Res. 30 (2018) 241-244.

84. Curtis NJ, West MA, Salib E, et al., Time from colorectal cancer diagnosis to laparoscopic curative surgery-is there a safe window for prehabilitation? Int J Colorectal Dis. 33 (2018) 979-983.

85. Hangaard Hansen C, Gögenur M, Tvilling Madsen M, et al., The effect of time from diagnosis to surgery on oncological outcomes in patients undergoing surgery for colon cancer: A systematic review, Eur J Surg Oncol. 44 (2018) 1479-1485.

86. Gurlit S, Gogol M, Prehabilitation is better than cure, Curr Opin Anaesthesiol. 32 (2019) 108-115.

87. Hulzebos EH, Helders PJ, Favié NJ, et al., Preoperative intensive inspiratory muscle training to prevent postoperative pulmonary complications in high-risk patients undergoing CABG surgery: a randomized clinical trial, JAMA. 296 (2006) 1851-1857.

88. Tsimopoulou I, Pasquali S, Howard R, et al., Psychological prehabilitation before cancer surgery: a systematic review, Ann Surg Oncol. 22 (2015) 4117-4123.

89. Levett DZ, Grimmett C, Psychological factors, prehabilitation and surgical outcomes: evidence and future directions, Anaesthesia. 74 (2019) Suppl 1:36-42. 

Valorization 

By law, Dutch universities have three main tasks; to educate at an academic level, to conduct scholarly research, and to ensure that research findings impact society. The latter is called valorization, with the following commonly used definition: "knowledge valorization is the process of creating value from knowledge, by making knowledge suitable and/or available for social and/or economic use, as well as by making knowledge suitable for translation into competitive products, services, processes, and new commercial activities" (adapted definition based on the National Valorization Committee 2011:8). This valorization chapter describes the possible value of the knowledge, products, and services developed and validated in this thesis, in which the author, as an "embedded scientist", combined daily clinical practice with the scientific evaluation and interpretation of clinical practice using usual care data for its analysis and the embedded research.

\section{BACKGROUND}

Nationwide, people are getting older, and the incidence of colorectal cancer will increase accordingly. With the advances in health care, more personalized treatment options are available, which lead to improvements in survival rate. Consequently, the amount of people living with (the consequences of) cancer and its corresponding treatment is increasing [1]. This thesis, as part of a nationwide program on perioperative care, aimed to examine the complete people's journey and treatment course of patients with colorectal cancer scheduled for elective surgery. Finally, the aim was to develop and implement optimal evidence-based physical therapy management, during the pre- and postoperative care pathway for patients with colorectal cancer, based upon the study findings. In order to support personalized health care, in which the appropriate individual physical therapy treatment is executed in joint coalition with the patient and his/her social relatives, at the right time, and in the right context.

This thesis provided innovative ingredients as; an overview of (the variation in) preand postoperative physical therapy management for patients opting for major elective abdominal surgery (2016-207) and the associations between preoperative performance parameters of physical fitness and postoperative outcomes (time to recovery of physical functioning, incidence and type of postoperative complications, length of hospital stay). Furthermore, it gave insight in changes in performance-based physical fitness and skeletal muscle measurements, before and after neoadjuvant chemoradiotherapy in single subjects with locally advanced rectal cancer, and in preoperative physical exercise training (prehabilitation) during neoadjuvant treatment for the same patient population. Finally, preparations for the evaluation of a personalized supervised home-based prehabilitation program were made by 
constructing a study protocol for the evaluation of the feasibility of the program and the monitoring of relevant parameters during the program. These ingredients cover the whole patient journey and provide insight in specific elements of performancebased physical functioning throughout this journey and the treatment course of patients with colorectal cancer.

\section{SOCIETAL RELEVANCE}

With the aging population, the incidence of diseases, including (colorectal) cancer is increasing $[2,3]$. Consequently, these changes challenge health care systems with increasing demands and the associated costs. For example, there is more need for long-term care and chronic disease management [4]. Moreover, elective surgery, the main treatment modality for colorectal cancer, has relatively high rates of morbidity and mortality [5,6]. A complicated postoperative course and/or suboptimal postoperative outcomes influence a patient's recovery of physical functioning and perceived health-related quality of life. Moreover, complications following colorectal cancer surgery are associated with a substantial increase in health care costs [7]. Hence, without a complicated postoperative period, patients can still experience a decrease in (independent) physical functioning and/or health-related quality of life due to the pathology, its treatment and the (function related) consequences of both. Optimizing the patient care pathway by targeting the right patients for preventive interventions may positively influence the treatment course and patient outcomes, as well as the corresponding health care profits and costs.

Inadequate levels of preoperative physical fitness are known to be associated with worse postoperative outcomes (e.g., higher risk for morbidity and mortality, higher risk for a prolonged length of hospital stay) after major abdominal surgery [8-10]. Preoperative interventions as physical exercise training and nutritional support can contribute to improving the level of preoperative physical fitness [11], which is likely to benefit postoperative (short-term) outcomes and (long-term) recovery of physical functioning and health-related quality of life [12], especially in high-risk patients. This latter will probably also improve health care values and at the same time lower the corresponding health care costs.

With adequate preoperative risk stratification, discrimination between the preoperative physical fitness levels of groups and - more relevant - probably also individual patients can be accomplished. Consequently, health care can be better adapted to the individual patient's needs (patient-tailored care). For example, patients with inadequate levels of physical fitness (high-risk patients) can be suggested to take part in supportive preoperative interventions. This will lead to a more efficient use of resources through the delivery of effective preventive interventions in the right patients, thereby of value in avoiding less effective high-cost interventions [13]. 
One aspect of this thesis was the implementation and execution of a preoperative screening of physical fitness followed by a personalized tailored advice on physical activity and physical fitness during the pre- and postoperative treatment course. Consequently, patients with colorectal cancer are more properly guided throughout their treatment course, focusing on reducing the loss of physical functioning associated with major surgery and facilitating a swift return to (vital) physical functioning in society during the pre- and postoperative phase. This can positively contribute to independent physical functioning and a better quality of life in patients with colorectal cancer. So, this preoperative screening of physical fitness can be of relevance to the patients.

\section{TARGET GROUPS}

The overall findings of this thesis are of value to patients and their social relatives, physical therapists, anesthesiologists, surgeons, other health care professionals, and (embedded) scientists.

\section{Individual patients}

In this thesis, (small) groups of individual patients were studied in the real-life context of our hospital with multiple measurements (e.g., pre- and postoperative, before and after neoadjuvant treatment). Results can be applied to individual patients and can contribute to personalized physical therapy care. Studies with small sample sizes or $\mathrm{n}=1$ studies contribute to evaluating interventions among specific patients with different characteristics in their specific context. Moreover, results from multiple $\mathrm{n}=1$ studies, gathered in a standardized way, can generate results applicable to a wider population [14]. By combining data from $n=1$ studies, common characteristics among patients who respond best to a specific intervention can be identified, at least that is the conviction that we would want to underline. For example, patients who respond best to a certain intervention may share genotypic, biomarker, clinical, or demographic characteristics [14]. Knowledge of these common aspects helps health care professionals by choosing a particular intervention for future patients with comparable characteristics. For the profession of physical therapy, such kind of research efforts may be helpful to enter the approach of personalized physical therapy, in parallel with the shift to personalized health. Future studies have to demonstrate the advantage of this approach, especially in the context of preoperative physical therapy as part of the prehabilitation concept. 


\section{(Inter)national colleagues and students}

The research findings were shared with direct colleagues at the department of physical therapy (Maastricht UMC+) and colleagues form other Dutch hospitals (community of practice) on a regular basis. Moreover, during this thesis project, there was a collaboration between the department of physical therapy of the Maastricht UMC+ and the physical therapists working in outpatient physical therapy practices $(n=20)$ within the catchment area of the hospital. One goal of this collaboration was to strengthen the link between outpatient physical therapy care and in-hospital physical therapy care, covering the complete patient journey. Hereto, physical therapy colleagues within and outside the hospital could learn from each other and benefit from the findings gathered during this thesis project. Besides, the colleagues in this collaboration were trained and educated in exercise physiology, preoperative home-based exercise training, motivational interviewing, monitoring progression during therapy sessions, and nutritional support during training. Furthermore, knowledge was shared with students from several educational levels (bachelor physical therapy, master physical therapy, master human movement sciences) with practical skill lessons, lectures, and thesis projects. Finally, knowledge gathered during this thesis was shared in (inter)national conferences concerning prehabilitation, physical therapy, nursing, and health sciences.

\section{Healthcare professionals}

This research project stimulated interdisciplinary teamwork, along the journey of each specific patient and his or her social relatives (e.g., partner, family), as several health care professionals worked together in this project. Surgeons, anesthesiologists, nurses, case managers (often a colorectal care nurse), dieticians, and physical therapists worked together during the whole journey per patient and during the research project period. Furthermore, findings and knowledge were presented several times during the project at hospital gatherings at the department of physical therapy, anesthesiology, surgery, and the nursing wards.

\section{Other scientists}

The (embedded) research procedures performed in this thesis might be of value for other (embedded) scientists. Data was collected during daily routines, which stimulates the direct translation from study results to daily practice and the people of interest [15]. However, research in a usual care setting has its limitations in terms of methodological quality (e.g., statistical power, bias and confounders, lack of a control group) and study results could also be biased by usual care protocols of the concerned hospital or by the people working in it. Another possible advantage of working as an embedded scientist is the direct connection between the health care professional 
(here the physical therapist) and academia (researchers). This connection ensures that embedded scientists stay up to date and remain a critical perspective towards the content and context of health care procedures [16]. It has been recommended that embedded scientists engage with other researchers doing similar work and share their knowledge [17]. During the whole research period of this thesis, gained knowledge was shared with several hospitals and colleagues in (inter)national communities of practice in order to learn from each other and exchange experiences.

\section{ACTIVITIES AND PRODUCTS}

The research and activities during this thesis project, as part of a nationwide program on perioperative care, contributed to the start of (sustainable) changes and implementations in a proactive care pathway for colorectal cancer in the Maastricht UMC+ concerning the preoperative phase, the postoperative phase, data collection, and the transfer of knowledge.

\section{Preoperative guidance for individual patients}

Currently, all patients diagnosed with colorectal cancer and opting for abdominal resection of the tumor can participate in a preoperative screening of physical fitness as part of risk stratification. Afterwards, patients receive an individualized advice concerning physical activity during the preoperative period with a referral for supervised home-based physical therapy when necessary (high-risk patients). This personal advice with guidance through the preoperative phase was a new aspect in the current care pathway in the Maastricht UMC+ and resulted from this embedded research project.

\section{Postoperative physical therapy management}

During the postoperative phase, recovery of physical functioning of patients is monitored more consistently and in more detail than before the start of this project. Recovery of physical functioning is measured on a daily base with the modified Iowa level of assistance scale (mILAS) and other clinimetric measurements applicable to the specific patient (e.g., timed up-and-go test, 2-minute walk test). These measurements are used to guide postoperative physical therapy treatment, in order to contribute to patient-tailored care.

\section{Contributions to data collection and infrastructure}

Data on clinical outcomes can be useful to measure the effects and value of the implementation of a certain advice, intervention, and/or treatment. Furthermore, it 
can contribute to a more detailed patient profile, and thereupon can be helpful in identifying both high- and low-risk patients. This thesis contributed to gathering and collecting relevant pre- and postoperative data, which can be used for evaluating daily clinical practice and guide pre- and postoperative physical therapy care.

Furthermore, the (usual care) data gathered in this thesis project is available for (larger) studies and for the build up of core sets of data in a national database. With this national database, usual care data can be evaluated faster and more accurate. Currently, the establishment of a connection between these core sets of data on physical functioning and the already existing medical databases (here, the Dutch Colorectal Audit of the Dutch Institute for Clinical Auditing) [18] is in progress. This will contribute to the evaluation of perioperative interventions and postoperative outcomes on a national level and provide better benchmark and mirror information for hospitals concerning the quality of care. Furthermore, data is used to educate physical therapists and other health care professionals at the Maastricht UMC+ with the main aim to deliver patient-tailored physical therapy treatment.

\section{Knowledge exchange between the research field and outpatient practices}

In the catchment area of the Maastricht UMC+, a network of trained, competent, and dedicated physical therapists working in outpatient physical therapy practices was developed, with the prospect of continuously optimizing this network for optimal preand postoperative physical therapy care over time. The physical therapists in this network were specifically trained in performing personalized preoperative physical therapy interventions for high-risk patients with colorectal cancer scheduled for elective resection. Moreover, they were, and are still being educated to guide these patients with functional task exercises and several monitoring methods at the patient's home. This education was based on previous scientific knowledge, previous research, and the nowadays practice-based experiences. Additionally, a decisionsupport guideline was developed for these physical therapists concerning preoperative physical exercise training. Within this ( $p$ )rehabilitation network, the aim is to establish a continuous exchange of (scientific) knowledge. The broader goal of this network is to make sure that patients are optimally prepared during the preoperative period in the right context, aiming to minimize the treatment-related loss of physical functioning and facilitation a complete and fast recovery. Furthermore, in cooperation with the colleagues of this network, the objective is to establish a long-term connection between hospital care and care in the outpatient physical therapy practices, to provide optimal care for patients throughout the complete continuum of care. 


\section{INNOVATION}

This thesis project was executed by embedded scientists. Embedded scientists continuously gather new insights and knowledge by collecting, analyzing, and interpreting data from patients and their dynamic contexts during daily practical and clinical routines $[15,19]$. This is in line with (continuous) comparative effectiveness research, which provides information needed by patients and health care professionals to make decisions and choose among alternative approaches in clinical care [20]. Continuous comparative effectiveness research includes the direct comparison of existing (health care) interventions, in order to determine which treatment and context works best, for which patient population, and under what circumstances [21]. Embedded research contributes to the transfer of new research knowledge into daily clinical practice, and vice versa.

\section{Embedded research: studying real-life practice, reducing research waste}

The transfer of knowledge from research into (changes in) clinical practice remains challenging [22-24], which can also be seen in chapter 2 of this thesis. Research waste arises when (large, expensive) studies with initially promising findings do not lead to improvements or changes in health care, which is an often-seen phenomenon in biomedical research [25]. Recently, this became the most worry of the entire life sciences and health scientific community. The gap between new knowledge from research and daily clinical practice cannot easily be explained [26], let alone how to overcome this gap. Hence, embedded research in the real-life context of the to be evaluated context may be able to contribute to reducing this gap, as research findings can probably enter the context of practice easier and faster, which may assist to reduce research waste. Moreover, with embedded research, the insight in exact issues affecting patients and caregivers increases [27]. Study aims and questions of embedded research arise from the local, mostly rather dynamic context, which probably makes them more relevant to daily clinical practice [28]. Final results might also be more easily incorporated into (changes in) daily practice. Embedding the research into daily clinical practice can be called innovative in the field of physical therapy and contributed to real-life data concerning the patients of our interest, and may therefore provide more context-valid outcomes then when investigated in a highly controlled research context [28]. All in all, the research in this thesis gave more insight in the people's journey for patients with colorectal cancer, contributing to more patient-tailored physical therapy care, focused on the P4 principles (predictive, preventive, personalized, and participatory) [29]. 


\section{SCHEDULE \& IMPLEMENTATION}

Several findings have already been incorporated into local daily practice. The work during this thesis project contributed to several adaptations in the care pathway for patients with colorectal cancer at the Maastricht UMC+. A preoperative screening of physical fitness with patient-tailored advice on preoperative physical activity and/or prehabilitation is a new component of the current care pathway. Furthermore, close and frequent postoperative monitoring of a patient's recovery of physical functioning by the physical therapist with help of the modified Iowa levels of assistance scale (mILAS) is rather new. The active approach of the patient and his/her support system and the proactive involvement of the physical therapist in the preoperative phase contributes to a more optimal preparation of patients and his/her support system before surgery. Patients and physical therapists can provide, evaluate and discuss information about the upcoming period, base their decisions and interventions upon these pieces of information, which is often appreciated by patients. Furthermore, preoperative performance of physical fitness can provide reference points for postoperative recovery of physical functioning for both patients and the physical therapist, which can help to manage optimal physical therapy preventive interventions and care.

\section{Future challenges}

An important future challenge is to perform adequately powered research in the reallife practice. This thesis showed that it is difficult to perform real-life practice research with the adequate power to strengthen the research conclusions. In order to accomplish this in the future, researchers should try to join national research developments. For example the development of personalized health. Personalized health aims to identify which approaches or interventions will be effective for specific patients. This requires the integration of diverse collections of data, generated in different hospitals and research centers [30]. For example the health research infrastructure (Health-RI), with the common goal to connect several data resources, thereby empowering researchers to develop better personalized medicine and health solutions [30]. When research institutes and hospitals work together in an (inter)national research infrastructure, this can contribute to adequately powered continuous comparative effectiveness research projects, which includes the comparison of existing interventions, in order to determine which treatment and context works best, for which individual patient and the patient's context [21].

A second challenge for the future is to maintain the changes made in the care pathway in joint coalition with patients with colorectal cancer, as well as with the structure of the care settings - peripheral and in the hospital - involved in this trajectory. The future will have to demonstrate whether the changes made are 
sustainable and will be further optimized, even though this thesis project has come to an end. The embedded scientist will continue working at the hospital aiming to further improve pre- and postoperative physical therapy care for patients undergoing colorectal resection. Furthermore, this project originated mainly from the Maastricht University side of the university complex, in coalition with the department of physical therapy of the Maastricht UMC+. When other health care professionals, besides the physical therapy, are also motivated for this topic and are able to encourage other professionals, this will positively contribute to maintaining the changes and expand the development concerning this topic. If not, the risk that the changes made during this project will fade out of the current system will be plausible. Besides, working together with people guiding the implementation, preferably during the complete project, may help to reach and keep (sustainable) changes present in daily practice [18]. Finally, it is challenging to continue with the work provided in this thesis, besides normal daily routines. There are still many questions left and the challenge is to stay critical towards work in daily practice and to be open for change, despite busy day schedules and routines of normal daily practice. 


\section{REFERENCES}

1. Ikkersheim D, Vernooij K, Wouters A, Toekomstverkenning betere kwaliteit van leven (KWF kankerbestrijding), KPMG Health (2018) 1-93.

2. World Health organization, Men, ageing and health: achieving health across the life span. https://www.who.int/ageing/publications/men/en/, 2019 (accessed January 2019).

3. World cancer research fund intrenational, worldwide cancer data: global cancer statistics for the most common cancers. https://www.wcrf.org/dietandcancer/ cancer-trends/worldwide-cancerdata, 2019 (accessed January 2019).

4. Heerkens $Y F$, de Weerd $M$, Huber $M$, et al., Reconsideration of the scheme of the international classification of functioning, disability and health: incentives from the Netherlands for a global debate, Disabil Rehabil. 40 (2018) 603-611.

5. Andreoni B, Chiappa A, Bertani E, et al., Surgical outcomes for colon and rectal cancer over a decade: results from a consecutive monocentric experience in 902 unselected patients, World J Surg Oncol. 5 (2007) 73.

6. Burns EM, Bottle A, Aylin P, et al., Variation in reoperation after colorectal surgery in England as an indicator of surgical performance: retrospective analysis of Hospital Episode Statistics, BMJ. 343 (2011) d4836.

7. Govaert JA, Fiocco M, van Dijk WA, et al., Costs of complications after colorectal cancer surgery in the Netherlands: Building the business case for hospitals, Eur J Surg Oncol. 41 (2015) 10591067.

8. West MA, Loughney L, Barben $C P$, et al., The effects of neoadjuvant chemoradiotherapy on physical fitness and morbidity in rectal cancer surgery patients, Eur J Surg Oncol. 40 (2014) 14211428.

9. Snowden $\mathrm{CP}$, Prentis J, Jacques B, et al., Cardiorespiratory fitness predicts mortality and hospital length of stay after major elective surgery in older people, Ann Surg. 257 (2013) 999-1004.

10. Levett $D Z$, Grocott MP, Cardiopulmonary exercise testing for risk prediction in major abdominal surgery, Anesthesiol Clin. 33 (2015) 1-16.

11. West MA, Wischmeyer $P E$, Grocott $M P$, Prehabilitation and nutritional support to improve perioperative outcomes, Curr Anesthesiol Rep. 7 (2017) 340-349.

12. Levett DZ, Edwards M, Grocott MP, et al., Preparing the patient for surgery to improve outcomes, Best Pract Res Clin Anaesthesiol. 30 (2016) 145-157.

13. Grocott MP, Edwards M, Mythen MG, et al., Peri-operative care pathways: re-engineering care to achieve the 'triple aim', Anaesthesia. 74 (2019) Suppl 1:90-99.

14. Lillie EO, Patay B, Diamant J, et al., The n-of-1 clinical trial: the ultimate strategy for individualizing medicine? Per Med. 8 (2011) 161-173.

15. Vindrola-Padros $C$, Pape T, Utley M, et al., The role of embedded research in quality improvement: a narrative review, BMJ Qual Saf. 26 (2017) 70-80.

16. Lewis SJ, Russell AJ, Being embedded: A way forward for ethnographic research, Ethnography. 12 (2011) 398-416.

17. Reiter-Theil $S$, Does empirical research make bioethics more relevant? "The embedded researcher" as a methodological approach, Med Health Care Philos. 7 (2004) 17-29.

18. Dutch Institute for Clinical Auditing (DICA) Leiden, DICA - DCRA. https://dica.nl/, 2019 (accessed February 2019).

19. Churruca K, Ludlow K, Taylor N, et al., The time has come: embedded implementation research for health care improvement, J Eval Clin Pract. 25 (2019) 373-380. 
20. Macleod MR, Michie S, Roberts I, et al., Biomedical research: increasing value, reducing waste. Lancet. 383 (2014) 101-104.

21. Greenfield S, Rich E, "Welcome to the journal of comparative effectiveness research, J Comp Eff Res. 1 (2012) 1-3.

22. Chew $S$, Institutionalising knowledge brokering as a sustainable knowledge translation solution in healthcare: how can it work in practice? Evid Policy. 9 (2013) 335-351.

23. Ward $V$, Knowledge brokering: the missing link in the evidence to action chain? Evid Policy. 5 (2009) 267-279.

24. Rowley E, Morriss R, Currie G, et al., Research into practice: collaboration for leadership in applied health research and care (CLAHRC) for Nottinghamshire, Derbyshire, Lincolnshire (NDL), Implement Sci. 7 (2012) 40.

25. Ferlie $E$, Knowledge mobilisation in healthcare: a critical review of health sector and generic management literature, Soc Sci Med. 74 (2012) 1297-1304.

26. Bannister J, Hardill I, Knowledge mobilisation and the social sciences: dancing with new partners in an age of austerity, Contempor Soc Sci. 8 (2013) 167-175.

27. Raad voor volksgezondheid en samenleving, Zonder context geen bewijs. https://www.raadrvs.nl/documenten/publicaties/2017/06/19/zonder-context-geen-bewijs, 2019 (accessed February 2019).

28. Bongers BC, Punt IM, van Meeteren NL, On "Prehabilitation: the emperor's new clothes or a new arena for physical therapists?" Lundberg M, Archer KR, Larsson C, Rydwik E, Phys Ther. 12 (2018) 127-130, Phys Ther. 99 (2019) 953-954.

29. Health research infrastructure, Empowering personalized medicine and health research. http://health-ri.org/, 2019 (accessed May 2019). 

Summary 

This thesis entitled "Preoperative optimization of physical functioning in patients with colorectal cancer" examines specific elements of performance-based physical functioning during the entire people's journey and treatment course of patients with colorectal cancer scheduled for elective colorectal resection.

Advances in public health and medicine, have led to improvements in overall life expectancy and consequently to an increase in the incidence of late life cancers, amongst others colorectal cancers. The primary treatment modality for colorectal cancer consists of abdominal resection, with or without (neo)adjuvant treatment. The disease and subsequent treatment can negatively affect physical fitness, skeletal muscle mass, daily functioning and quality of life. Physical fitness and skeletal muscle mass are important factors influencing completion of (neo)adjuvant treatment and the complete recovery of physical functioning after major abdominal surgery. Furthermore, there is a consistent positive relation between these parameters and postoperative morbidity and mortality. In the period before the definitive surgical procedure, there is the opportunity to optimize the patient's physical functioning with preoperative interventions, to provide patients with a higher psychophysiological buffer to better withstand the stress of hospitalization and surgery, which might minimize the decline of physical functioning following surgery. Before starting these preventive interventions (generally known under the umbrella term "prehabilitation"), there should be an adequate prediction procedure of hospitalization- and surgeryrelated risks. Preferably, this entails a personalized risk identification and recognition procedure to identify a patient's risks for all types of complications in the pre- and postoperative period. Hence, vulnerable high-risk patients can be distinguished from low-risk patients, and, more importantly, adequate preventive care strategies can be tailored together with the individual (high-risk) patient and his or her social support system. All of these ingredients integrated in one overall conceptual rationale are presented in chapter $\mathbf{1}$.

In chapter 2, the overall self-reported professional care content and betweenhospital variation of perioperative physical therapy management in patients undergoing elective major abdominal surgery for gastrointestinal malignancies was investigated in Dutch hospitals (2016-2017) on the base of a national survey (response rate of 79\%). Furthermore, daily clinical physical therapy management was compared with the nowadays-advised state-of-the-art pre- and postoperative physical therapy. The chapter shows that there is a wide degree of reported variability between Dutch hospitals regarding pre-and postoperative clinical physical therapy practice for patients opting for major abdominal surgery. Three classes of daily clinical physical therapy practices were identified, differing in adherence to the evidence provided in the literature. Of the 63 hospitals, one (1.6\%) reported $80 \%$ compliance to evidence-based practice, whereas 15 hospitals (23.8\%) reported $50-75 \%$ 
compliance, and 47 hospitals (74.6\%) reported $10-50 \%$ compliance to evidencebased practice. Further translation of key research findings into clinical physical therapy practice is advised, especially for the hospitals in which the physical therapist is not involved preoperatively.

Chapter 3 evaluated whether preoperative performance parameters of physical fitness were associated with postoperative outcomes in patients with colorectal cancer scheduled for elective resection. This study revealed associations between preoperative physical fitness and postoperative outcomes in patients with colorectal cancer scheduled for resection. Surgical procedure and identification via the national screening program were related to lower stages of TNM-scores and a faster postoperative time to recovery of physical functioning as measured with help of the modified Iowa levels of assistance (mILAS), namely 1 to 3 days faster. Furthermore, the preoperative level of functional exercise capacity (measured with the incremental shuttle walk test), functional mobility (measured with the timed up-and-go test), and the level of perceived fatigue (measured with the multidimensional fatigue index questionnaire) were independent predictors of a faster postoperative time to recovery of physical functioning. A complete preoperative evaluation was consequently considered valuable for patients, their social relatives, and caregivers, and can ensure a quick start for appropriate preoperative interventions when and where necessary.

Chapter 4 aimed to evaluate the change in performance-based physical fitness and computed tomography derived skeletal muscle measurements, before and after neoadjuvant chemoradiotherapy in patients with locally advanced rectal cancer scheduled for elective resection. This chapter revealed a statistically significant reduction in aerobic capacity and skeletal muscle mass following neoadjuvant chemoradiotherapy, in which a clinically relevant decline was observed in approximately $50 \%$ of the patients. These findings were also reported in other studies. A large inter-individual variation concerning alterations in performance-based physical fitness, skeletal muscle mass, and the experience of negative side-effects was observed. Data showed that patients with a reduced physical fitness after completion of the neoadjuvant chemoradiotherapy had an increased risk for experiencing negative side effects during the treatment. The large variability between subjects requires all the more a highly personalized individual risk-based proactive and preferably preventive treatment approach throughout the entire perioperative trajectory. The latter ideally includes frequent monitoring of physical fitness performances of the patient (also named "titration") and probably also interventions whenever these parameters show scores beneath a certain crucial level.

The primary aim of chapter $\mathbf{5}$ was to determine the feasibility of a supervised outpatient physical exercise training program during neoadjuvant chemoradiotherapy in patients with rectal cancer. Secondly, the preliminary effectiveness of this physical 
exercise training program on physical fitness (functional exercise capacity and muscle strength), fatigue, and quality of life of individual patients was studied. This study revealed that a supervised outpatient physical exercise training program for patients with locally advanced resectable rectal cancer during neoadjuvant chemoradiotherapy is feasible for the patients that decided to participate. Thirteen of the 20 eligible patients $(65 \%)$ were willing to participate and able to complete the program with a progressive increase in training load, without any adverse events. Furthermore, participating in the moderate-intensity physical exercise training program during neoadjuvant chemoradiotherapy led in these patients to a statistically significant increase in leg and arm muscle strength, and a statistically non-significant increase in functional exercise capacity after $12 \pm 3$ weeks of training. Fatigue and quality of life remained relatively stable during the program, despite the progression of individual exercise intensity. Because of its potential effects and the low feasibility of the recruitment and exercise procedures, we should look for means to have more if not all patients to participate and profit from this potentially valuable approach.

Chapter 6 contains a protocol for assessing the feasibility (adherence/compliance, adverse events, and patient appreciation) of a four-week supervised and personalized home-based prehabilitation program for high-risk patients scheduled for major elective abdominal resection. Prehabilitation programs have shown to improve physical fitness before surgery of the participating patients. However, not all the patients do participate on the base of all sorts of reasons, and results about postoperative benefits are inconclusive. Furthermore, the content and execution of prehabilitation programs are heterogeneous and the selection of in- and exclusion criteria is diverse. In this one-group pre-test post-test pilot study, objectively identified high-risk patients participating in a supervised and personalized homebased prehabilitation program (approximately four weeks, on average 12 sessions in total) will be selected, in order to increase inclusion and adherence/compliance rates compared to hospital-based prehabilitation programs or home-based programs without supervised sessions. Results of this exploratory pilot study can subsequently be used to further optimize the program and will promote a sustainable, cost-effective implementation.

In chapter 7 the main findings and limitations of the studies presented in this thesis are discussed in the context of current knowledge and literature. Furthermore, general conclusions and recommendations, as well as directions for further research are presented 

Samenvatting 

Dit proefschrift getiteld "Preoperative optimization of physical functioning in patients with colorectal cancer" evalueert specifieke elementen van fysiek functioneren gemeten met performance testen tijdens de people's journey en het behandeltraject van patiënten met colorectaal kanker die gepland staan voor een electieve abdominale resectie.

Verbeteringen in de zorg hebben de afgelopen jaren geleid tot een toename van de algehele levensverwachting. Samen met de invoering van het bevolkingsonderzoek voor colorectaal kanker is hierdoor de incidentie van colorectaal kanker de afgelopen jaren gestegen. De belangrijkste behandeloptie voor colorectaal kanker bestaat uit een chirurgische abdominale resectie van de tumor met of zonder (neo)adjuvante behandeling (chemo- en/of radiotherapie). De ziekte zelf en de behandeling ervan kunnen aërobe capaciteit, de spierkracht en de kwaliteit van leven negatief beïnvloeden. Het behouden van aërobe capaciteit en spierkracht is echter zeer belangrijk voor een normaal en volledig herstel van het fysiek functioneren na een grote abdominale resectie, alsook voor het volhouden en het volgens het initiele protocol voltooien van additionele chemo- en/of radiotherapie. Tegenwoordig komt er steeds meer aandacht voor prevalidatie, wat meestal bestaat uit preoperatieve fysieke training, eventueel gecombineerd met voedingsinterventies. Prevalidatie kan bijdragen aan het opbouwen van (extra) adequate preoperatieve reserves van aërobe capaciteit en spierkracht om beter met de stress van de electieve abdominale resectie om te kunnen gaan. Vervolgens zouden deze reserves positief kunnen bijdragen aan het postoperatieve herstel van het fysiek functioneren. Voordat prevalidatie kan starten, zou een goede risicopredictie plaats moeten vinden om patiënten met een hoger risico op (postoperatieve) complicaties op voorhand te identificeren. Hiermee kunnen adequate preventieve strategieën toegepast worden bij patiënten die dit nodig hebben. Al deze aspecten zijn beschreven in hoofdstuk 1.

In hoofdstuk 2 werd de werkwijze van Nederlandse ziekenhuis fysiotherapeuten (in 2016-2017) onderzocht. De inhoud en variatie tussen ziekenhuizen met betrekking tot pre- en postoperatieve fysiotherapie voor patiënten die een abdominale resectie ondergaan omwille van gastrointestinale maligniteiten werd geëvalueerd (response graad van 79\%). Daarnaast werd de inhoud van deze dagelijkse pre- en postoperatieve fysiotherapeutische zorg vergeleken met de huidige evidence-based literatuur. De resultaten lieten zien dat er een grote hoeveelheid variatie bestaat tussen Nederlandse ziekenhuizen met betrekking tot de pre- en postoperatieve fysiotherapeutische zorg voor deze patiëntengroep. Er zijn drie categorieën van dagelijks fysiotherapie management geïdentificeerd, die verschillen in de uitvoering van evidence-based fysiotherapie zoals beschreven in de literatuur. Van de 63 ziekenhuizen, rapporteerde één ziekenhuis (1.6\%) 80\% compliance met de evidencebased literatuur. Een groep van 15 ziekenhuizen (23.8\%) rapporteerde 50-75\% 
compliance, en 47 ziekenhuizen (74.6\%) rapporteerden 10-50\% compliance met de evidence-based literatuur. Verdere vertaling van belangrijke evidence-based bevindingen naar de dagelijkse klinische fysiotherapie wordt geadviseerd, vooral voor de ziekenhuizen waar de fysiotherapeut nog niet of nauwelijks betrokken is in de preoperatieve fase van het zorgpad.

Hoofdstuk 3 bestudeerde of preoperatieve fysieke fitheid en fysiek functioneren gerelateerd zijn aan postoperatieve uitkomsten bij patiënten met colorectaal kanker die een abdominale resectie ondergaan. Deze studie liet zien dat er een relatie is tussen preoperatieve fysieke fitheid en fysiek functioneren en postoperatieve uitkomsten. De chirurgische procedure (een laparoscopische of open resectie) en de (vroege) identificatie bij het nationaal bevolkingsonderzoek waren gerelateerd aan lagere tumorstadia en aan een sneller postoperatief herstel van functioneren. Het postoperatief herstel van functioneren was 1 tot 3 dagen sneller, gemeten met de modified Iowa levels of assistance scale (mILAS). Daarbij is er een relatie gevonden tussen preoperatieve functionele inspanningscapaciteit (gemeten met de incremental shuttle walk test), preoperatieve functionele mobiliteit (gemeten met de timed-upand-go test), en ervaren vermoeidheid (gemeten met een vermoeidheidsvragenlijst) en een sneller postoperatief herstel van fysiek functioneren. Een complete preoperatieve evaluatie is waardevol voor patiënten en zorgprofessionals en kan bijdragen aan een snelle(re) start van gepaste pre- en postoperatieve interventies.

Hoofdstuk 4 evalueerde de verandering in fysieke fitheid (aërobe capaciteit en spierkracht), gemeten met performance testen, en spiermassa, beoordeeld op computertomografie (CT) scans, voor en na neoadjuvante chemoradiotherapie in individuele patiënten met rectumkanker. Deze studie liet zien dat er een statistisch significante afname was in aërobe capaciteit en spiermassa na neoadjuvante chemoradiotherapie $(n=25)$, waarbij een klinisch relevante afname werd geobserveerd in $50 \%$ van de patiënten. Deze bevindingen worden ook ondersteund door eerdere studies. Er was echter sprake van grote interindividuele variatie, aangezien de resultaten lieten zien dat sommige patiënten minder goed presteren op de testen van fysieke fitheid na de neoadjuvante therapie, terwijl andere patiënten nagenoeg gelijke prestaties lieten zien. Ook werd er variatie gevonden in het ervaren van negatieve bijwerkingen van de behandeling. Patiënten met een afname in fysieke fitheid na het afronden van de neoadjuvante chemoradiotherapie rapporteerden meer negatieve bijwerkingen van de behandeling. De grote variatie tussen individuele patiënten vraagt om een gepersonaliseerde, idealiter preventieve behandelmethode tijdens het gehele behandeltraject, waarbij fysiotherapie nuttig kan zijn om tijdens de behandeling fysieke fitheid te monitoren en indien nodig te trainen voor betere patiënt gerelateerde uitkomsten, zoals het ervaren van bijwerkingen en het continueren van de neoadjuvante behandeling. 
Het hoofddoel van de studie in hoofdstuk $\mathbf{5}$ was het bepalen van de haalbaarheid van een gesuperviseerd fysiek trainingsprogramma tijdens neoadjuvante chemoradiotherapie voor patiënten met rectumkanker. Ten tweede, werd ook geëvalueerd of functionele inspanningscapaciteit, ervaren vermoeidheid en kwaliteit van leven veranderden tijdens het trainingsprogramma. Dit programma vond poliklinisch plaats tijdens de periode van neoadjuvante chemoradiotherapie op de afdeling fysiotherapie van het Maastricht UMC+, op de dagen dat de patiënten ook in het ziekenhuis moesten zijn voor de radiotherapie. De resultaten lieten zien dat een gesuperviseerd trainingsprogramma voor deze patiënten tijdens behandeling veilig en haalbaar was, voor het deel van de patiënten dat besloot om deel te nemen. Het was echter moeilijk om alle patiënten die daadwerkelijk in aanmerking kwamen voor het programma, te includeren. 13 van de 20 beschikbare patiënten (65\%) wilde deelnemen en hebben het programma voltooid met een progressie in trainingsintensiteit, zonder ongewenste voorvallen of negatieve bijwerkingen. Daarbij lieten de resultaten een statistisch significante toename zien in been- en armspierkracht, en een statistisch niet-significante toename in functionele inspanningscapaciteit na een periode van gemiddeld $12 \pm 3$ weken trainen, terwijl de ervaren vermoeidheid en kwaliteit van leven geen veranderingen liet zien in dezelfde periode. Omdat dit programma potentiele positieve effecten heeft, dient er in de toekomst gekeken te worden naar manieren om elke patiënt te laten deelnemen aan deze preoperatieve preventieve benadering.

Met het oog op betere participatie van patiënten die in aanmerking komen voor preoperatieve interventies, beschrijft hoofdstuk 6 een studieprotocol voor de evaluatie van een gesuperviseerd thuisprevalidatieprogramma voor patiënten die gepland staan voor een electieve abdominale resectie omwille van gastrointestinale maligniteiten. Deze pilotstudie beoogt de haalbaarheid van een thuisprevalidatieprogramma voor objectief geïdentificeerde hoog-risico patiënten te evalueren, in een één-groep pre-post pilotstudie. Het prevalidatieprogramma duurt 4 weken. Eerdere prevalidatieprogramma's hebben laten zien dat deze effectief zijn in het verbeteren van de preoperatieve fysieke fitheid bij patiënten die gepland staan voor een grote abdominale resectie. Echter, doen vaak niet alle patiënten mee en zijn de resultaten over de postoperatieve voordelen (afname van het risico op complicaties, sneller herstel van het fysiek functioneren, kortere opnameduur) van deze programma's niet eenduidig. Ook zijn de inhoud, uitvoering en de te hanteren in- en exclusiecriteria van deze programma's heterogeen en divers. Het studieprotocol beschrijt een in de thuiscontext gesuperviseerd prevalidatieprogramma, om zo de inclusie te verhogen en uitval te beperken vergeleken met prevalidatieprogramma's die in het ziekenhuis plaatsvinden of die geen gesuperviseerde sessies bevatten. De resultaten van deze 
exploratieve pilotstudie kunnen gebruikt worden om het programma verder te optimaliseren en een duurzame, kosteneffectieve implementatie te faciliteren.

In hoofdstuk 7 worden de bevindingen en limitaties van de eerder beschreven studies bediscussieerd in de context van de huidige kennis en literatuur. Daarbij worden algemene conclusies en aanbevelingen gedaan alsook voorschriften voor toekomstig onderzoek. 


Dankwoord 

Een promotietraject doorloop je nooit alleen. Op de voor- en achtergrond zijn er verschillende mensen bij betrokken geweest die ik hier graag wil bedanken.

Op de eerste plaats wil ik mijn promotieteam bedanken; Prof. dr. Nico van Meeteren, Prof. dr. Wolfgang Buhre en dr. Bart Bongers. Nico, je bent altijd bereikbaar voor vragen of feedback. Via de mail, via de telefoon of face to face. Ik heb veel gehad aan deze begeleiding en daarbij leerde jij mij veel op het gebied van netwerken en communicatie. Bij onze (wekelijkse) overleggen gingen Christel en ik vaak met een hele to do lijst de deur uit. Dit was soms frusterend, maar nu achteraf weet ik: dit was altijd voor een hoger doel. Prof. Buhre, Wolfgang, hoewel het contact niet heel intensief was, heb ik veel gehad aan de begeleiding. Constructieve overleggen over de voortgang en altijd nuttige feedback op mijn artikelen. U zorgde ervoor dat ik altijd kritisch bleef richting het schrijfwerk en het werk op de klinische afdelingen. Bij de bijeenkomsten van de anesthesiologie was ik eigenlijk een beetje een vreemde eend in de bijt, maar ook dat was voor mij erg leerzaam en heeft mijn netwerk doen vergroten. Bart, als dagelijkse begeleider stond jij altijd voor me klaar. Niets was te veel en de geschreven artikelen werden tot in detail door jou nagekeken. Geen punt of komma zag jij over het hoofd. Ook was het fijn om met jou zaken opnieuw te reflecteren en te evalueren. Jij leerde me keuzes maken en prioriteiten stellen. Door jouw vele connecties kwam er altijd weer een oplossing voor de verschillende problemen en heb ik heel veel kunnen leren.

Ook wil ik graag Ilona Punt bedanken. Ilona, op papier officieel niet bij mijn promotie betrokken, maar ook bij jou kon ik altijd terecht voor advies en feedback of een gezellig praatje. Daarbij was het fijn om verschillende zaken te bediscussiëren, te reflecteren en samen te werken in het netwerk van BiBo (Better in, Better out).

Graag wil ik de leden van de beoordelingscommissie bedanken voor het beoordelen van mijn proefschrift en alle co-auteurs voor de fijne samenwerking bij de verschillende artikelen. Een aantal co-auteurs noem ik graag bij naam; Judith de Vos, Laurents Stassen en Iris Rutten, bedankt voor de feedback op de artikelen, hulp bij de dataverzameling en de nuttige overleggen. Daarnaast zijn er in het Maastricht UMC+ ook anderen die mij verder hebben geholpen met mijn onderzoek; Christel Gielen, Eveline Smeets en Lieke Corpelijn; bedankt voor alle hulp bij het doorsturen van patiënten, het meedenken en het uitvoeren van praktische werkzaamheden! Ook wil ik graag projectgroep BiBo oncologie bedanken, voor alle hulp en input bij dit project.

Ook bedank ik de afdeling Epidemiologie, Zowel in UNS 40 als in DEB 1. Net voordat ik klaar zou zijn met dit boekje, zijn we toch nog verhuisd naar DEB 1 en horen we er helemaal bij. Rob de Bie en Conny de Zwart, bedankt voor alle hulp 
tijdens mijn promotietraject. Van vragen over methodologie tot het regelen van een treinkaart. Het werd allemaal zeer gewaardeerd.

En dan natuurlijk alle collega's van de afdeling Fysiotherapie van het Maastricht UMC+. Er werden vaak grapjes gemaakt over "de wetenschappers" die alles altijd net weer een beetje anders wilden. Maar jullie waren altijd bereid om mee te denken en uit te helpen waar dat kon. Ik heb me altijd zeer thuis gevoeld in dit team (en nog steeds!). George, halverwege mijn promotietraject ging jij met pensioen en kregen we een nieuwe baas. Jij was altijd erg betrokken en zonder jou had deze promotie niet kunnen gebeuren. Toen ik na mijn tijdelijke contracten weg moest van de afdeling fysiotherapie, heb jij er mede voor gezorgd dat ik terug mocht komen. Ook daarna kon ik altijd bij je terecht. Overleg met de verpleegkundigen, aanschaf van nieuwe spullen, herinrichting van de ruimtes, niets was te gek. Merci! Rob, jij nam het stokje van George over. Ook vanuit jou was er altijd interesse voor ons onderzoek. Ton, als hoofd onderzoek van onze afdeling, en nu bijzonder hoogleraar Klinische Fysiotherapie, stond jij altijd voor mij klaar. Het begon met mijn afstudeerscriptie van de master en daarna stond ik nog regelmatig voor je deur met verschillende vragen. Daarbij ging jij mijn kritieken niet uit de weg. Bedankt!

Onze afdeling fysiotherapie is niets zonder het secretariaat (Victoire, Ilse en Wilke) en de afdelingsassistenten (Frank en Margot). Ontzettend veel telefoontjes hebben jullie voor mij aangenomen, berichtjes genoteerd en vragen van patiënten beantwoord. Daarbij kon het onderzoek niet doorgaan zonder een goede stopwatch, saturatiemeter, klappers en papieren. En natuurlijk de testfiets, die altijd in goede staat moest zijn en regelmatig van locatie wisselde. Dit alles had nooit gekund zonder jullie! Bedankt!

Een aantal collega's van de afdeling fysiotherapie wil ik hier graag in het bijzonder noemen: de PhD-collega's: Danielle, Hanneke, Marissa en Anouk, bedankt voor de nuttige discussies en leuke afleidingen buiten het werk. Maud en Niels, mede betrokken bij onze metingen rondom BiBo voor de oncologische buikchirurgie en altijd bereid om mee te denken en uit te helpen. Yvonne, een spontane stedentrip heeft geleid tot meer leuke uitjes, gezellige etentjes en goede gesprekken. Erg waardevol tijdens een drukke PhD, bedankt! En dan natuurlijk nog Christel, die hier zeer zeker niet mag ontbreken! Mijn BiBo onderzoeksmaatje en kamergenootje! Veel hebben we samen opgezet en uitgevogeld. Daarbij heb ik veel van je geleerd, onder andere computervaardigheden in excel en powerpoint, maar ook over doorzettingsvermogen en enthousiasme! Elkaar uithelpen met de metingen en nuttige discussies voeren, dat is zo waardevol! Collega's en patiënten haalden ons vaak door elkaar, omdat we met dezelfde zaken bezig waren. Ook buiten werk heb ik veel leuke dingen met jou beleefd (pizza eten, samen op cursus, of tripjes naar de ikea). Jij stond en staat altijd voor me klaar! Waarvoor heel veel dank!! 
Natuurlijk wil ik ook alle patiënten bedanken. Zonder jullie zijn er geen gegevens en zonder gegevens is er niets om onderzoek mee te doen. Ook zijn er tijdens mijn promotietraject verschillende bachelor- en master- studenten geweest, die ik heb mogen begeleiden tijdens de stage. Bedankt voor het meehelpen met de dataverzameling voor dit onderzoek. Het was leuk en leerzaam om jullie te begeleiden. Verder wil ik de BiBo groep in Utrecht bedanken voor de leerzame bijeenkomsten, goede discussies en nuttige feedback op mijn presentaties en artikelen. Ook wil ik hier mijn nieuwe collega's van Fysiotherapie Team $\mathbf{C}$ van de Fontys Eindhoven noemen. Bedankt voor het warme welkom en de nieuwe uitdagingen!

In dit dankwoord wil ik ook graag mijn vriendinnen bedanken. Sommige van jullie ken ik al heel lang en anderen van jullie ken ik sinds ik in Maastricht woon. Regelmatig vroegen jullie hoe het ervoor stond met mijn onderzoek. Daarbij zorgden jullie vaak voor aangename afleidingen tijdens mijn PhD-tijd met uitstapjes, feestjes en gezellige avonden. Een aantal van jullie zijn ook PhD-student (geweest) wat zorgde voor fijne gesprekken en herkenbare situaties. In het bijzonder wil ik hier noemen; Wai Yan, mijn maatje, in alles! Samen studeren, samen volleyballen, kletsen, uitgaan, lachen en mijn voorbeeld op het gebied van promoveren! Leonie, tijdens onze tijd in Maastricht, woonde jij maar één straat bij me vandaan. Wat ertoe leidde dat we heel vaak samen waren (aan de wandel op weg naar een mc flurry, samen bankhangen en natuurlijk in de kantine na een training of wedstrijd). Bedankt voor alle goede gesprekken en je kritische blik! Sanne, het begon met volleybal maar tegenwoordig treffen we elkaar bij pilates of zwemmen of gaan we bij elkaar eten. Altijd een fijne afleiding en we voerden regelmatig goede discussies over onderzoek en werk. Daarbij was jouw boekje een grote bron van inspiratie. Bedankt!

Iris en Marleen, de laatste meisjes van GW die nog in Maastricht wonen. Samen op stap, lunchen of gezellig bijkletsen. Dat is erg waardevol en dat we nog maar veel leuke dingen samen mogen ondernemen. Ook mogen mijn studiegenootjes en vriendinnen Ruth, Sanne, Daphne, Fabienne en Marieke niet ontbreken in dit dankwoord. Bedankt voor jullie interesse in mijn onderzoek en alle gezelligheid buiten werktijd! Liza en Martine, mijn middenmaatjes van de laatste jaren bij Fyrfad. Bedankt voor de gezelligheid, dagjes uit en natuurlijk bedankt voor het samen volleyballen! En dan nog mijn vriendinnen die ik al langer ken, Danique, Marlon, Lonneke en Ilse, mijn studiegenootjes (zowel van gezondheidswetenschappen als van fysiotherapie), mijn teamgenootjes van het volleyballen (van alle seizoenen) en natuurlijk mijn familie; Bedankt voor jullie interesse in mijn promotie onderzoek! 
Tot slot mogen in dit dankwoord zeker niet ontbreken pap, mam, Linda en David. Ook al begrepen jullie niet altijd helemaal waar ik mee bezig was, dit boekje was nooit tot stand gekomen zonder jullie. Bedankt voor jullie onvoorwaardelijke steun, interesse en aangename afleidingen in mijn vrije tijd! 


Curriculum Vitae 

Aniek Heldens was born on 18 December 1988 in Eindhoven, the Netherlands. After completing secondary school (atheneum, Elzendaal College, Boxmeer, 2001-2007) she studied Health Sciences at Maastricht University (2007-2010) and Physical therapy at Zuyd University of Applied Sciences in Heerlen (2010-2013). She started with the master Human Movement Sciences (Biology of Human Performance and Health) at Maastricht University in 2013, from which she graduated in 2014. During and after finishing this master year, she has been working as a physical therapist in the Maastricht University Medical Center+ (Maastricht UMC+).

In March 2015 she started her PhD project at the department of Epidemiology (Maastricht University) combined with working as a physical therapist at the department of Physical Therapy of the Maastricht UMC+. In the PhD project she worked under the supervision of Prof. dr. Nico van Meeteren and Prof. dr. Wolfgang Buhre, with the daily supervision of dr. Bart Bongers. During this PhD project, she was involved in the monitoring and evaluation of patient characteristics, physical functioning, preoperative physical exercise training and postoperative outcomes in patients with colorectal cancer scheduled for elective abdominal resection.

Currently, Aniek is working as a physical therapist at the Maastricht UMC+ and as a teacher at the Fontys University of Applied Sciences in Eindhoven. 

List of publications 



\section{LIST OF PUBLICATIONS}

\section{Accepted manuscripts}

Heldens AF, Bongers BC, de Vos-Geelen J, van Meeteren NL, Lenssen AF. Feasibility and preliminary effectiveness of a physical exercise training program during neoadjuvant chemoradiotherapy in individual patients with rectal cancer prior to major elective surgery. European Journal of Surgical Oncology 2016;42:1322-30.

Heldens AF, Bongers BC, de Vos-Geelen J, van Meeteren NL, Lenssen AF. Reply to: Exercising patient-centeredness in prehabilitation programs. European Journal of Surgical Oncology 2016;43:511-12.

Heldens AF, Bongers BC, Lenssen AF, Stassen LP, Buhre WF, van Meeteren NLU. The association between performance parameters of physical fitness and postoperative outcomes in patients undergoing colorectal surgery: An evaluation of care data. European Journal of Surgical Oncology 2017;43:2084-92.

Heldens AF*, van Beijsterveld CA*, Bongers BC, van Meeteren NL. Variation in preoperative and postoperative physical therapist management for patients opting for elective abdominal surgery. ${ }^{*}$ Shared first authorship. Physical therapy 2019 (in press).

\section{Submitted manuscripts}

Heldens AF, Bongers BC, de Vos-Geelen J, Minis-Rutten IJ, Stassen LP, Buhre WF, van Meeteren NL. Clinical course of physical fitness and skeletal muscle mass during neoadjuvant chemoradiotherapy in patients with locally advanced rectal cancer: an observational longitudinal study of 25 single subjects.

Heldens AF, Bongers BC, van Beijsterveld CA, Melenhorst J, Dejong $\mathrm{CH}$, den Dulk M, Coolsen M, Stassen LP, Buhre WF, van Meeteren NL. Study protocol of a onegroup pre-test post-test pilot study to evaluate a home-based prehabilitation program for high-risk patients undergoing major elective abdominal surgery. 